Illinois State University

ISU ReD: Research and eData

Theses and Dissertations

8-15-2013

\title{
An Exploration of Relations among the Wechsler Scales, the Woodcock-Johnson III Cognitive and Achievement Batteries, and Mental Health Measures in a Sample of College Students with Suspected Disabilities
}

C. Lee Affrunti

Illinois State University, lee@clarea.com

Follow this and additional works at: https://ir.library.illinoisstate.edu/etd

Part of the Psychology Commons

\section{Recommended Citation}

Affrunti, C. Lee, "An Exploration of Relations among the Wechsler Scales, the Woodcock-Johnson III Cognitive and Achievement Batteries, and Mental Health Measures in a Sample of College Students with Suspected Disabilities" (2013). Theses and Dissertations. 29.

https://ir.library.illinoisstate.edu/etd/29

This Dissertation is brought to you for free and open access by ISU ReD: Research and eData. It has been accepted for inclusion in Theses and Dissertations by an authorized administrator of ISU ReD: Research and eData. For more information, please contact ISUReD@ilstu.edu. 


\section{AN EXPLORATION OF RELATIONS AMONG THE WECHSLER SCALES, THE WOODCOCK-JOHNSON III COGNITIVE AND ACHIEVEMENT}

BATTERIES, AND MENTAL HEALTH MEASURES

IN A SAMPLE OF COLLEGE STUDENTS

WITH SUSPECTED DISABILITIES

\section{LEE AFFRUNTI}

262 Pages

December 2013

This dissertation describes cognitive, achievement, and mental health relations in an existing university dataset and proposes a model describing the influence of services at the university's disabilities resource center. 


\title{
AN EXPLORATION OF RELATIONS AMONG THE WECHSLER SCALES, THE WOODCOCK-JOHNSON III COGNITIVE AND ACHIEVEMENT
}

\author{
BATTERIES, AND MENTAL HEALTH MEASURES \\ IN A SAMPLE OF COLLEGE STUDENTS \\ WITH SUSPECTED DISABILITIES
}

\section{LEE AFFRUNTI}

262 Pages

December 2013

This dissertation reports results of analyses of an archival dataset created at a large Midwestern public university, where staff at the university's resource center for students with disabilities conduct neuropsychological evaluations of students suspected of psychological disabilities, learning disabilities, or both. To explore the relations among the variables, analyses included standardized cognitive and achievement test scores, psychological rating scales results, resource center service utilization, and seven to eight consecutive semesters of grade-point average information of approximately 1292 students evaluated from 2000 to 2012. Descriptions of the cognitive and achievement variables are provided for the largest demographic and diagnostic groups. Demographic groups include male, female, Caucasian, African American, Latino, and Asian/Indian students; diagnostic groups include attention deficit hyperactivity disorder predominantly combined type (ADHD-C), attention deficit hyperactivity disorder predominantly inattentive type (ADHD-I), anxiety, depression, verbal learning disability 
(VLD), nonverbal learning disability (NVLD), foreign language learning difficulty (FLLD), and "No Diagnosis." Results of analyses indicated that 1) The model of latent cognitive abilities suggested by this sample's results largely matches, with minor variations, models proposed by researchers who have analyzed the standardization samples of the intelligence and achievement batteries used in this investigation;2) Cognitive-achievement relations, as suggested by results obtained on two standardized tests of cognitive abilities and selected subtests of a standardized achievement test, generally match, with minor variations, results of past analyses of college students; and 3) A proposed model of the influence of disability services utilization on grade-point average slope was not supported by analyses using structural equation modeling. Latent growth curve analyses indicated, however, that students' grade point average slopes improved after neuropsychological evaluation. 

AN EXPLORATION OF RELATIONS AMONG THE WECHSLER SCALES, THE WOODCOCK-JOHNSON III COGNITIVE AND ACHIEVEMENT

BATTERIES, AND MENTAL HEALTH MEASURES

IN A SAMPLE OF COLLEGE STUDENTS

WITH SUSPECTED DISABILITIES

C. LEE AFFRUNTI

A Dissertation Submitted in Partial
Fulfillment of the Requirements
for the Degree of
DOCTOR OF PHILOSOPHY
Department of Psychology
ILLINOIS STATE UNIVERSITY

2013 
UMI Number: 3609692

All rights reserved

INFORMATION TO ALL USERS

The quality of this reproduction is dependent upon the quality of the copy submitted.

In the unlikely event that the author did not send a complete manuscript and there are missing pages, these will be noted. Also, if material had to be removed, a note will indicate the deletion.

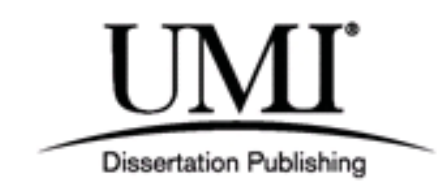

UMI 3609692

Published by ProQuest LLC (2014). Copyright in the Dissertation held by the Author.

Microform Edition (C) ProQuest LLC.

All rights reserved. This work is protected against unauthorized copying under Title 17, United States Code

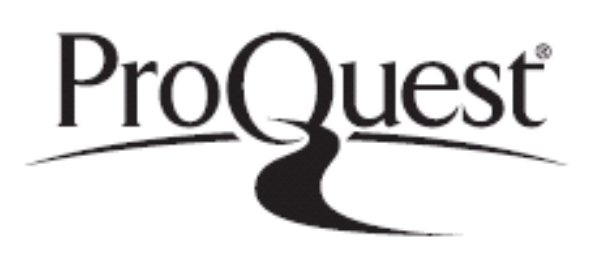

ProQuest LLC.

789 East Eisenhower Parkway

P.O. Box 1346

Ann Arbor, MI 48106 - 1346 
(C) 2013 C. Lee Affrunti 
AN EXPLORATION OF RELATIONS AMONG THE WECHSLER SCALES, THE WOODCOCK-JOHNSON III COGNITIVE AND ACHIEVEMENT

BATTERIES, AND MENTAL HEALTH MEASURES

IN A SAMPLE OF COLLEGE STUDENTS

WITH SUSPECTED DISABILITIES

C. LEE AFFRUNTI

DISSERTATION APPROVED:

$\overline{\text { Date } \quad \text { W. Joel Schneider, Chair }}$

Date Renée M. Tobin

$\overline{\text { Date } \quad \text { Matthew Hesson-McInnis }}$

Date Kimberly D. Collins 


\section{ACKNOWLEDGMENTS}

It is with my deepest appreciation that I acknowledge the considerable and judicious assistance of the Illinois State University school psychology faculty and my committee members in helping me complete this dissertation. Specifically, I am profoundly grateful for my program advisor, Steven Landau, for his continuing guidance and resolute support throughout my academic career. My successful completion of this dissertation also depended greatly on my committee members' unique and valued contributions. I extend my heartfelt thanks to Kim Collins for allowing me to examine her extraordinary dataset and for sharing her unique insights into the remarkable students who comprise it; to Renée Tobin, for mentoring my earliest research experiences and inspiring me to think critically about my research and its implications for theory and practice; to Matthew Hesson McInnis, for sympathetically guiding my statistics education and continually believing in my ability to learn; and especially to my chair, Joel Schneider, for generously imparting to me his considerable knowledge, infinite patience, and unfailing support while shepherding me through the dissertation process.

This dissertation is dedicated to my family: my husband, Robert, and our daughter, Erin. I am forever grateful for their unwavering belief in my ultimate success and their steadfast encouragement when the going got tough. They are truly my rock.

C.L.A. 


\section{CONTENTS}

Page

ACKNOWLEDGMENTS $\quad$ i

CONTENTS $\quad$ ii

TABLES $\quad$ V

FIGURES $\quad$ X

CHAPTER

I. RESEARCH AIM AND HYPOTHESES 1

Introduction $\quad 1$

Research Aim 5

Hypotheses Regarding Cognitive, Achievement, and
Mental Health Relations

II. REVIEW OF RELATED LITERATURE 7

$\begin{array}{ll}\text { General Literature Review } & 7\end{array}$

History of Psychometric Intelligence Theory $\quad 7$

Galton and the Anthropometric Laboratory 8

J. M. Cattell and the Empirical Study of "Mental Tests" 9

Spearman and the Two-Factor Theory of Intelligence 9

Thurstone and Primary Mental Abilities $\quad 10$

Vernon and Additional Analytical Developments 11

Horn and Cattell's $g_{f}-g_{c}$ Theories $\quad 12$

Carroll and the Three-Stratum Theory 13

Cattell-Horn-Carroll (CHC) Theory 14

$\mathrm{CHC}$ and the Current Study's Test Development 19

Cognitive-Achievement Relations and Factor Structures 21 
The Purported Benefits of Adding Subtest Scores to a Cognitive Battery 35

Disability Service Utilization on Achievement 37

The Present Study 45

Research Aim $\quad 45$

$\begin{array}{ll}\text { ADHD } & 47\end{array}$

Learning Disabilities $\quad 47$

Emotional Disorders 48

Foreign Language Learning Difficulties $\quad 48$

Hypothesis 1 - Cognitive-Achievement Tests and

CHC Factor Analysis 49

Hypothesis 2 - Cognitive-Achievement Relations 57

Hypothesis 3 - Consistent Incremental Validity of Additional Test Scores Regardless of Subtest

Score Variability $\quad 63$

Hypothesis 4 - The Benefit of Service Utilization on GPA 64

$\begin{array}{ll}\text { III. METHOD } & 67\end{array}$

$\begin{array}{ll}\text { Participants } & 67\end{array}$

$\begin{array}{ll}\text { Measures } & 71\end{array}$

Woodcock-Johnson III (WJ III) 72

Wechsler Adult Intelligence Scales (WAIS) 76

Conners' Adult ADHD Rating Scales (CAARS) 87

Symptom Checklist-90-R (SCL-90-R) 91

Beck Depression Inventory - Second Edition (BDI-II) 93

IV. RESULTS 96

$\begin{array}{ll}\text { Research Aim - Descriptive Analyses } & 97\end{array}$

$\begin{array}{lr}\text { Gender } & 112\end{array}$

Ethnicity 119

Primary Diagnosis 140

Foreign Language Learning Difficulty 162

iii 
Hypothesis 1 - Factor Analyses of the WAIS-III, WAIS-IV, and WJ III

Model 1 - Hierarchical and Bifactor $g$ Models

196

Model 2 - Hierarchical and Bifactor $g_{f} g_{c}$ Models

206

Hypothesis 2 - Cognitive-Achievement Relations

Hypothesis 3 - Consistent Incremental Validity of Additional

Test Scores Regardless of Subtest Score Variability

Hypothesis 4 - The Benefit of Service Utilization on GPA

\section{DISCUSSION}

Research Aim - Descriptive Analyses of the Demographic and Diagnostic Groups

Gender

Ethnicity

Primary Diagnosis

Hypothesis 1 - Factor Analyses of the WAIS-III/IV and WJ III Cognitive and Achievement Subtests

Hypothesis 2 - Cognitive-Achievement Relations

Hypothesis 3 - Incremental Validity with Subtest Variability

Hypothesis 4 - The Benefits of Service Utilization

Study Strengths and Limitations

General Conclusions and Directions for Future Study 


\section{TABLES}

Table

Page

1. Comparable Cognitive Abilities Subtests and Their Broad-Narrow Factors

2. Groups Profiled by Cognitive, Achievement, and Diagnostic Tests

3. Diagnoses Represented in the Sample $(N=1,982)$

4. Relations of Wechsler and WJ III COG Tests and CHC Abilities

5. Relations of WJ III ACH Tests, Related Curricular Areas, and CHC Abilities

6. Subtest/Cluster Median Reliability Statistics of the WJ III NU Cognitive and Achievement Subtests

7. Internal Reliability Coefficients for CAARS:S:L and CAARS:O:L For 18-29 Year Olds

8. Mean Inter-Item Correlations for CAARS:S:L and CAARS:O:L for 18-29 Year Olds

9. Test-Retest Correlations for CAARS:S:L and CAARS:O:L - Ages And Sexes Combined

10. Standard Error of Measurement Scores for CAARS:S:L and CAARS:O:L for 18-29 Year Olds

11. Standard Error of Prediction (SEP) Scores for CAARS:S:L and CAARS:O:L for 18-29 Year Olds

12. Convergent/Discriminant Validity Correlations between BDI-II and Other Measures

13. Means, Standard Errors, Standard Deviations, Skew and Kurtosis for WAIS-III FSIQ and Subtests of the WAIS-III Calibration Dataset 
TABLES, continued

Table

Page

14. Means, Standard Errors, Standard Deviations, Skew and Kurtosis for WJ III Cognitive GIA and Subtests of the WAIS-III Calibration Dataset

15. Means, Standard Errors, Standard Deviations, Skew and Kurtosis for WJ III Achievement Subtests of the WAIS-III Calibration Dataset

16. Means, Standard Errors, Standard Deviations, Skew and Kurtosis for Subtests of the WAIS-IV Calibration Dataset

17. Means, Standard Errors, Standard Deviations, Skew and Kurtosis for WJ III Cognitive GIA and Subtests of the WAIS-IV Calibration Dataset

18. Means, Standard Errors, Standard Deviations, Skew and Kurtosis for WJ III Achievement Subtests of the WAIS-IV Calibration Dataset

19. Means, Standard Errors, Standard Deviations, Skew and Kurtosis for FSIQ and Subtests of the Combined WAIS-III/IV Calibration Dataset

20. Means, Standard Errors, Standard Deviations, Skew and Kurtosis for WJ III Cognitive GIA and Subtests of the Combined WAIS-III/IV Calibration Dataset

21. Means, Standard Errors, Standard Deviations, Skew and Kurtosis for the WJ III Achievement Tests of the WAIS-III/IV Combined Calibration Dataset

22. Means, Standard Errors, Standard Deviations, Skew and Kurtosis for Rating Scale Items of the Self- and Other-Rated CAARS of the WAIS-III Calibration Dataset

23. Means, Standard Errors, Standard Deviations, Skew and Kurtosis for Rating Scale Items of the Self- and Other-Rated CAARS from the WAIS-IV Calibration Dataset

24. Means, Standard Errors, Standard Deviations, Skew and Kurtosis for Rating Scale Items of the Self- and Other-Rated CAARS from the WAIS-III/IV Combined Calibration Dataset 
TABLES, continued

Table

Page

25. Means, Standard Errors, Standard Deviations, Skew and Kurtosis for the BDI-II Total Score and SCL-90-R GSI of the WAIS-III Calibration Dataset

26. Means, Standard Errors, Standard Deviations, Skew and Kurtosis for the BDI-II Total Score and SCL-90-R GSI of the WAIS-IV Calibration Dataset

27. Means, Standard Errors, Standard Deviations, Skew and Kurtosis for the BDI-II Total Score and SCL-90-R GSI of the WAIS-III/IV Combined Calibration Dataset

28. Means, Standard Errors, Standard Deviations, and t-test Results of the Combined WAIS-III/IV FSIQ and Subtests by Gender

29. Means, Standard Errors, Standard Deviations, and t-test Results of the Combined WAIS-III/IV Dataset's WJ III GIA and Subtests by Gender

30. Means, Standard Errors, Standard Deviations, and t-test Results of the Combined WAIS-III/IV Dataset's WJ III Achievement Subtests by Gender

31. Means, Standard Errors, Standard Deviations, and t-test Results of the CAARS Self- and Other-rated Scales of the Combined WAIS-III/IV Dataset by Gender

32. Means, Standard Errors, Standard Deviations, and t-test Results of the Combined WAIS-III/IV Dataset's BDI-II Total Score and SCL-90-R GSI by Gender

33. Cross-tabulations of Diagnosis and Gender from the WAIS-III/IV Combined Dataset

34. WAIS-III/IV Combined Dataset ANOVA Results for WAIS-III/IV FSIQ and Subtests by Ethnicity

35. WAIS-III/IV Combined Dataset ANOVA Results for the WJ III Cognitive GIA and Subtests by Ethnicity

36. WAIS-III/IV Combined Dataset ANOVA Results for the WJ III Achievement Subtests by Ethnicity 
TABLES, continued

Table

Page

37. WAIS-III/IV Combined Dataset ANOVA Results for the CAARS

Self- and Other-rated Scales by Ethnicity

38. WAIS-III/IV Combined Dataset ANOVA Results for the BDI-II Total Score and SCL-90-R GSI by Ethnicity

39. Cross-tabulations of Diagnosis and Ethnicity from the

WAIS-III/IV Combined Dataset

40. WAIS-III/IV Combined Dataset ANOVA Results for WAIS-III/IV FSIQ and Subtests by Primary Diagnosis

41. WAIS-III/IV Combined Dataset ANOVA Results for the WJ III Cognitive GIA and Subtests by Primary Diagnosis

42. WAIS-III/IV Combined Dataset ANOVA Results for the WJ III Achievement Subtests by Primary Diagnosis

43. WAIS-III/IV Combined Dataset ANOVA Results for the CAARS Self- and Other-rated Scales by Primary Diagnosis

44. WAIS-III/IV Combined Dataset ANOVA Results for the BDI-II Total Score and SCL-90-R GSI by Primary Diagnosis

45. Means, Standard Errors, Standard Deviations, and t-test Results for Combined WAIS-III/IV FSIQ and Subtests by Foreign Language Learning Difficulty (FLLD)

46. Means, Standard Errors, Standard Deviations, and t-test Results for Combined WAISIII/IV Dataset's WJ III GIA and Subtests by Foreign Language Learning Difficulty (FLLD)

47. Means, Standard Errors, Standard Deviations, and t-test Results for Combined WAIS-III/IV Dataset's WJ III Achievement Subtests by Foreign Language Learning Difficulty (FLLD)

48. Means, Standard Errors, Standard Deviations, and t-test Results of the CAARS Self- and Other-rated Scales of the Combined WAIS-III/IV Dataset by Foreign Language Learning Difficulty (FLLD) 
TABLES, continued

Table

Page

49. Means, Standard Errors, Standard Deviations, and t-test Results of the Combined WAIS-III/IV Dataset's BDI-II Total Score and SCL-90-R GSI by Foreign Language Learning Difficulty (FLLD)

50. Names and Abbreviations of Analyzed Cognitive and Achievement Subtests

51. Calibration Dataset Correlation Matrix for Cognitive and Achievement Variables

52. Validation Dataset Correlation Matrix for Cognitive and Achievement Variables

53. Full Combined Dataset Correlation Matrix for Cognitive, Achievement, and Mental Health Variables

54. Factor Loadings for WAIS-III/IV Combined Calibration Dataset

55. Hypotheses Testing $-g$ Hierarchical and Bifactor Models

56. Hypotheses Testing $-g_{f^{-}} g_{c}$ Hierarchical and Bifactor Models

57. Hypotheses Testing - $g$ Hierarchical and Bifactor Models as Achievement Predictors 


\section{FIGURES}

Figure $\quad$ Page

1. Measurement model of WAIS-III and WJ III COG with CHC broad factors and narrow factors measured by least two subtests 52

2. Measurement model of WAIS-IV and WJ III COG with CHC broad factors and narrow factors measured by least two subtests 53

3. WAIS-III, WJ III COG, and WJ III ACH in $g_{f^{-}} g_{c}$ measurement model 55

4. WAIS-IV, WJ III COG, and WJ III ACH in $g_{f} g_{c}$ measurement model 56

5. Hypothesis $2 \mathrm{a}(1)$ : For reading and writing achievement, there is no mediation of basic skills in the relations between cognitive abilities and advanced skills

6. Hypothesis $2 \mathrm{a}(2)$ : For mathematics achievement, there is no mediation of basic skills in the relations between cognitive abilities and advanced skills

7. Hypothesis $2 b(1)$ : For reading and writing achievement, the extent to which basic skills mediate the relations between cognitive abilities and advanced skills was tested

8. Hypothesis $2 \mathrm{~b}(2)$ : For mathematics achievement, the extent to which basic skills mediate the relations between cognitive abilities and advanced skills was tested

9. Hypothesized model of service utilization's effects on GPA slope

10. Final hierarchical $g$ model of WAIS-III/IV and WJ III COG with $\mathrm{CHC}$ broad and narrow factors

11. Final bifactor $g$ model of WAIS-III/IV and WJ III COG with CHC broad factors

12. Final hierarchical $g_{f} g_{c}$ model of WAIS-III/IV and WJ III COG with $\mathrm{CHC}$ broad and narrow factors 
FIGURES, continued

Figure

Page

13. Final bifactor $g_{f} g_{c}$ model of WAIS-III/IV and WJ III COG with $\mathrm{CHC}$ broad factors

14. Final hierarchical cognitive-achievement structural model of WAIS-III/IV, WJ III COG, and WJ III ACH subtests with significant $\mathrm{CHC}$ broad and narrow factors

15. Final bifactor cognitive-achievement structural model of WAIS-III/IV, WJ III COG, and WJ III ACH subtests with significant $\mathrm{CHC}$ factors

16. Service utilization model using latent growth curve analysis

17. GPA trajectories by whether a student received an accommodation letter 


\section{CHAPTER I}

\section{RESEARCH AIM AND HYPOTHESES}

\section{Introduction}

For psychoeducational assessment in the United States, the state of the art is in a state of flux and uncertainty. Many assessment professionals and researchers had long been dissatisfied with the previously prevailing model of learning disability identification when it was updated with a set of procedures known as Response-to-Intervention (RTI; IDEA, 2004). The old model required students with a specific learning disability to demonstrate a discrepancy between "ability," as measured by intelligence tests, and "achievement" in one or more of the seven domains of oral expression, listening comprehension, written expression, basic reading skill, reading comprehension, mathematical calculation, and mathematical reasoning, as measured by grades and standardized achievement tests. This procedural definition of specific learning disability identification has been criticized on theoretical, practical, and moral grounds (Fuchs \& Fuchs, 2006).

The goal of RTI practitioners is to provide universal screening and "scientific, research-based" instruction and interventions based on consistently monitored student data, and proponents argue that RTI provides earlier intervention than would typically occur using the ability-achievement discrepancy model (Buffum, Mattos, \& Weber, 
2010). With the advent of the Individuals with Disabilities Education Improvement Act (IDEA) reauthorization of 2004 and the Response-to-Intervention (RTI) model, students are evaluated for a specific learning disability whenever they fail to make sufficient progress in one or more of the eight achievement areas of oral expression, listening comprehension, written expression, basic reading skill, reading fluency, reading comprehension, mathematics calculation, or mathematics problem solving. "Sufficient progress" is determined using early universal screening measures and students' responses to scientific, research-based intervention. Because an additional inclusionary criterion implemented by IDEA 2004 requires that a student's underachievement also not be due to a lack of appropriate instruction, a determination evaluation must also include evidence that the student was provided appropriate instruction by qualified instructors in regular education settings. Additionally, the evaluation must include data-based documentation of achievement assessments repeated at regular intervals and reflecting formal assessment of student progress during instruction (IDEA, 2004).

Critics note that there is no clear and theoretically sound definition and procedure of diagnosing learning disabilities within the RTI framework (Reynolds \& Shaywitz, 2009) and that RTI researchers have been unable to identify who will respond well to intervention reliably (Fuchs \& Fuchs, 2006). The 2006 IDEA regulations allow for both the old discrepancy-based definition of a specific learning disability and an RTI-based definition. It also allows a "third method" for identifying a specific learning disability, which is becoming increasingly popular with school practitioners who are looking for a way to address some of RTI's limitations (Flanagan, Fiorello, \& Ortiz, 2010). This model emphasizes a "processing strengths and weaknesses" approach in which people 
with specific learning disability have average or better general cognitive ability but have academic deficiencies caused by specific cognitive ability weaknesses. Regardless of the model used, assessment goals at the K-12 level have always included finding optimal interventions and accommodations to maximize student learning (McCloskey, Whitaker, Murphy, \& Rogers, 2012).

The evaluation picture has not evolved for college students as it has for students in primary and secondary school. A postsecondary setting encompasses myriad curricula and would therefore make implementation of an RTI paradigm nearly impossible. In addition, K-12 public schools abide by laws defined by IDEA 2004 and Section 504 of the Rehabilitation Act of 1973, whereas postsecondary institutions follow the laws under Section 504 and the Americans with Disabilities Act (ADA; National Joint Committee on Learning Disabilities; NJCLD, 2007b). As a result of these practical considerations and differing legal requirements, colleges continue to follow a discrepancy model to diagnose learning disabilities, a model that requires individualized testing with standardized intelligence and achievement tests. Typical college-level evaluations also employ structured clinical interviews and the administration of several rating scales to assess for psychological disorders.

Adhering to Section 504 and ADA, colleges then offer accommodations, but not interventions, for diagnosed learning problems, although many institutions offer several forms of learning assistance, including tutoring, coaching, and counseling (NJCLD, 2007b). Accommodations for college students can include additional exam time, a distraction-free exam space, alternative exam formats, classroom notetakers, facultyprovided written course notes or assignments, help with learning strategies, study, time 
management, or organization, and adaptive technology (Raue \& Lewis, 2011).

The extent to which assessment results can reliably predict who will benefit from which accommodations remains unclear, however, especially with regard to students diagnosed with attention-deficit hyperactivity disorder (ADHD; DuPaul, Weyandt, O’Dell, \& Varejao, 2009). Complicating this conundrum is the fact that the training required to conduct evaluations is expensive and time intensive. Clinicians are either professionals who have already completed graduate school or advanced graduate students who have completed one or more classes in psychoeducational assessment. Further, the typical evaluation requires approximately 8-12 hours to complete, a considerable length of professional time with questionable utility in terms of determining whether and how someone will receive accommodations, be referred to a university health center for medication, or both.

Regardless of questions related to evaluation, colleges enroll many students with disabilities, and the number of college students with psychological and learning disabilities is rising. In 2008 , nearly $88 \%$ of all U. S. degree-granting institutions enrolled approximately 700,000 students with disabilities, or about $11 \%$ of all postsecondary students (Raue \& Lewis, 2011). Between 2000 and 2008, the percentage of students with mental, emotional, or mood disorders jumped $70 \%$ to account for $24 \%$ of students with disabilities by 2008. The proportion of students with ADHD increased $285 \%$ during this time, to $19 \%$ of students with disabilities, and the percentage of students with specific learning disabilities rose $56 \%$, to $8.9 \%$. It would be prudent to learn how institutions of higher education might better serve the needs of a group that accounts for nearly $6 \%$ of the total college population. 


\section{Research Aim}

One aim of this study is to explore a dataset of evaluation results of students with suspected disabilities from a large, Midwestern, public university. This dataset was created as a composite of more than 10 years of data collected from evaluating a diverse group of students who sought assistance from the university's disabilities resource center. Comprehensive evaluations included standardized, individually administered tests that measured various aspects of cognitive functioning, including language development, abstract reasoning, and short and long-term memory. Evaluations also included standardized tests of achievement, including tests of reading comprehension, spelling, and mathematics. In addition, students undergoing evaluation completed several rating scales measuring symptoms related to ADHD and other psychological difficulties. Descriptions of the relations among these measures were provided with respect to the largest demographic and diagnostic groups in the dataset, although no explanatory effort was made with respect to diagnosis because all the examined measures were used in the diagnostic process.

\section{Hypotheses Regarding Cognitive, Achievement, and Mental Health Relations}

By exploring the results of confirmatory factor analyses and structural equation modeling of the cognitive, achievement, and mental health variables in this dataset, four hypotheses were proposed that consider the relations among the measures analyzed in this study. First, I hypothesized that confirmatory factor analyses of the cognitive and achievement variables in this dataset would support current Cattell-Horn-Carroll (CHC) theory's factor model of latent cognitive abilities. In addition, I explored Cattell's (1947) $g_{f^{-}} g_{c}$ theory of cognitive abilities through confirmatory factor analyses with this dataset. 
Next, I hypothesized that the cognitive-achievement relations in this sample would largely match the cognitive-achievement relations found in other studies but might differ somewhat because most other studies (e.g., Floyd, McGrew, \& Evans, 2008; McGrew \& Wendling, 2010) examined younger students than were examined in this sample. In addition, results from analyses of this sample are likely to vary from others' results because other studies' findings vary among each other. Third, I hypothesized that there would be consistent incremental validity of additional test scores in an administered battery regardless of subtest score variability. This hypothesis was made possible by the exploration of two relatively complete cognitive batteries and the expectation that they would yield hypothesized broad and narrow factors for analysis. Finally, I hypothesized that, through structural equation modeling, I would determine the extent to which students' cognitive abilities, achievement, initial psychological functioning, and use of the disabilities resource center's services predicted academic outcomes as measured by the student's grade point average (GPA) slope for at least three semesters postassessment. 


\section{CHAPTER II}

\section{REVIEW OF RELATED LITERATURE}

\section{General Literature Review}

This chapter's purpose is to provide a comprehensive review of the literature pertinent to this study. The topics of this review include 1) a history of psychometric intelligence theory leading to current CHC theory (McGrew, 1997, 2005, 2009; Schneider \& McGrew, 2012) and test development, 2) an examination of cognitiveachievement relations from a $\mathrm{CHC}$ perspective, 3) an overview of cross-battery assessment (Flanagan, Alfonso, \& Ortiz, 2012) and its role in enhancing composite validity, and 4) a review of the relations between student characteristics and service utilization in influencing student achievement.

\section{History of Psychometric Intelligence Theory}

Relevant to the current study is a description of the development of modern intelligence assessment, including the theoretical work that has influenced the development of the intelligence tests used in this analysis: the Woodcock-Johnson III Tests of Cognitive Abilities and Tests of Achievement (WJ III COG, WJ III ACH; Woodcock, McGrew, \& Mather, 2001b, 2001a), and the Wechsler Adult Intelligence Scales, Third and Fourth Editions (WAIS-III and WAIS-IV; Wechsler, 1997b, 2008).

The Chinese are the first known people to have instituted, over 2,000 years ago, 
tests that measured human abilities for the purposes of classifying people into groups to determine optimal employment fit (Kamphaus et al., 2005). Modern inquiries into the nature and structure of intelligence, however, likely had their roots with Charles Darwin (1809-1882), whose theory of evolution fostered the British philosopher Herbert Spencer's (1920-1903) coining of the phrase, "survival of the fittest" (p. 444; Spencer, 1864). Both Spencer and Darwin framed intelligence in terms of one's capacity to adapt to one's environment, and they surmised that this capacity was largely genetic (Wasserman, 2012).

Galton and the Anthropometric Laboratory. After reading Darwin's Origin of Species, Darwin's half-cousin, Francis Galton (1822-1911), became interested in heredity and its role in the individual differences of human abilities Galton's contributions to the study of intelligence began in his Anthropometric Laboratory where he sought to measure, among other aspects of the human body, physical efficiency that purportedly improved academic performance (Wasserman, 2012). Galton's interests generated prolific empirical testing to inform the nature versus nurture debate (he advocated "nature" over "nurture"). In addition, Galton's intensive experimentation fostered great advances in psychometrics including test battery and survey questionnaire development, the use of control groups, and the development of statistical methods such as regression and correlation (Wasserman, 2012). Because he was able to procure large samples, Galton was the first to reveal the relevance of the normal distribution curve to human attributes such as intelligence, as well as being the first to use percentile scores to measure a person's standing relative to that distribution (Plucker, 2003). 


\section{J. M. Cattell and the empirical study of "mental tests." As prodigious as}

Galton's contributions were in the field of psychometrics, his legacy was substantially enhanced by his students, including James M. Cattell (1860-1944), who coined the term "mental test" (Cattell, 1890; Schneider \& McGrew, 2012). Before working in Galton's lab, Cattell studied human reaction times under William Wundt in Germany (Schneider \& McGrew, 2012; Wasserman, 2012). With his interest and insistence on empirical inquiry rather than on Wundt's preference for a "reliance on experimenter introspection" (Wasserman, 2012, p. 9), Cattell moved psychology into the realm of hard science. Cattell's atheoretical emphasis on empirical testing, however, focused primarily on simple human processes such as reaction time and sensory discrimination (Wasserman, 2012). Cattell's approach eventually lost favor when a subsequent study (Sharp, 1899) criticized Cattell's lack of explanatory theory and foretold the promise of testing more complex abilities, such as various aspects of memory, attention, and creativity, as more salient indicators of intelligence. Further, Wissler (1901), a graduate student at Columbia University, discovered that Cattell's tests had few correlations with each other or with academic achievement which, at that time, was considered an important facet of intelligence (Spearman, 1904; Wasserman, 2012). Although Sharp's and Wissler's studies were found to be flawed, anthropometric testing nevertheless receded into history, although some of the primary processes of interest to Cattell, such as reaction time, are currently being reconsidered as relevant to a more complete understanding of intelligence (Schneider \& McGrew, 2012; Wasserman, 2012).

Spearman and the two-factor theory of intelligence. As another student under Wundt, Charles Spearman (1863-1945) also concerned himself with individual 
differences in human abilities. Spearman's (1904) development of factor analysis was a landmark event in the history of intelligence testing and propelled the psychometric field into a theory-based endeavor (Wasserman \& Tulsky, 2005). After noticing that tests of mental ability were positively correlated, Spearman (1904) proposed that some general factor accounted for this phenomenon. He named the factor " $g$ " for "general intelligence" and posited that $g$ represented the shared variance of all intelligence tests and would reflect how someone would perform across test batteries (Kranzler, 1997).

Because correlations were dissimilar across tests, Spearman hypothesized that there were varying amounts of $g$ represented within each measure and that tests with higher $g$ loadings would be more highly correlated (Brody, 1999). Spearman also noted that the imperfect correlations among the various tests within a battery suggested that there were processes specific to each test, which he labeled "s," that were not accounted for by the general factor $g$ (Wasserman \& Tulsky, 2005). Spearman's two-factor theory of intelligence hypothesized that both $g$ and $s$ combine to manifest one's mental ability on an intelligence test (Spearman, 1904).

Spearman was reticent to admit that $s$ factors represented any more than testspecific variance. However, later in his life and following much debate with fellow intelligence researchers including Edward Thorndike and Godfrey Thompson, Spearman acknowledged that tests that are similar to each other with respect to content or process tend to have higher correlations than can be accounted for by $g$ alone (Brody, 1999; Wasserman \& Tulsky, 2005).

Thurstone and primary mental abilities. Advances in statistical methodology, including multi-factor analysis, allowed L. L. Thurstone (1887-1955) to find separate 
general factors that disputed Spearman's hypothesis of only a single general factor (Thurstone, 1938; Wasserman, 2012; Wasserman \& Tulsky, 2005). Thurstone posited that $g$ was chiefly the product of the relation between eight primary independent factors which he called Primary Mental Abilities: verbal comprehension, word fluency, number fluency, induction, speed of judgment, memory, spatial relations, and perceptual speed (Schneider \& McGrew, 2012; Wasserman, 2012). Thurstone eventually acknowledged $g$ 's existence hierarchically above his primary mental abilities, noting that despite finding separate factors, he also found that measures tended to be positively correlated with each other (Brody, 1999). Thurstone continued to disagree with Spearman about $g$ 's relative importance, however, believing the establishment of cognitive profiles using primary mental abilities would better explain strengths and weaknesses in intellectual abilities even in people with similar overall ability scores (Schneider \& McGrew, 2012;

Wasserman, 2012).

Vernon and additional analytical developments. Spearman's and Thurstone's work precipitated further advances in analytic methods which yielded a strong theoretical basis for the development of an intelligence factor structure. In the years following Thurstone's establishment of multi-factor analysis, Philip Vernon (1905-1987) proposed the first hierarchical model of intelligence with $g$ being dominant over the lower-order factors, verbal/educational abilities and spatial/mechanical abilities (Wasserman, 2012). This dichotomy would eventually lend itself to the later development of the separate verbal and performance components of the Wechsler intelligence batteries (Wasserman, 2012).

The Educational Testing Service contributed another important milestone in 
psychometric development by encouraging the development of a standard set of reference tests to provide factor markers for analysis studies. This effort resulted in the evidence of more than 60 possible primary mental abilities and well-replicated common factors abilities (Schneider \& McGrew, 2012).

Horn and Cattell's $\boldsymbol{g}_{\boldsymbol{f}} \boldsymbol{g}_{\boldsymbol{c}}$ theories. Raymond Cattell (1905-1998) studied under Spearman and used Thurstone's multi-factor analytic methods with the primary mental abilities and well-replicated common factors abilities datasets to hypothesize that $g$ was actually two separate general factors: " $g_{c}$," crystallized intelligence that can be measured by tests that assess what one has learned via one's culture or formal education, and " $g f$," general fluid intelligence defined as a novel reasoning facility that is more neurologically based and relatively independent of culture or education (Cattell, 1943; Schneider \& McGrew, 2012; Wasserman, 2012). Cattell (1943) also postulated that $g_{f}$ and $g_{c}$ are highly correlated, giving rise to Spearman's $g$, because $g_{f}$ supports the development of $g_{c}$ through investment. Cattell's investment theory posited that higher levels of $g_{f}$ would optimize the time and effort involved in learning and ultimately enhance $g_{c}$, whereas lower levels of $g_{f}$ would make learning more effortful and ultimately dampen $g_{c}$ (Cattell, 1943; Schneider \& McGrew, 2012; Wasserman, 2012).

Cattell's $g_{f} g_{c}$ theory was empirically tested and supported by one of Cattell's students, John Horn (1928-2006), who demonstrated the different developmental trajectories of $g_{c}$ and $g_{f}$, lending credence to the separateness of these factors (Horn \& Blankson, 2012; Horn \& Cattell, 1966, 1982). Horn also revised Cattell's theory to include several broad cognitive abilities, or second-order factors, instead of the two primary factors $g_{c}$ and $g_{f}$ (Horn \& Blankson, 2012). These lower-order factors included 
more narrowly defined notions of fluid (Gf) and crystallized (Gc) intelligence, short-term apprehension and retrieval (SAR), fluency of retrieval from long-term storage (TSR), processing speed (Gs), and visual processing (Gv; Schneider \& McGrew, 2012). A convention was adopted following Horn's work on extended $g_{f}-g_{c}$ theory to label secondorder factors with an uppercase "G" followed by the initial(s) of the specific broad factor, to distinguish them from Cattell's original $g_{c}$ and $g_{f}$ primary factors as well as from Spearman's primary general ability factor which would retain an italicized lowercase " $g$ " (Schneider \& McGrew, 2012; Wasserman, 2012). Called the extended $g_{f}-g_{c}$ theory, Horn's theory was eventually expanded to include nine broad abilities including auditory processing $(\mathrm{G} a)$, quantitative ability $(\mathrm{G} q)$, and reading and writing facility ( $\mathrm{G} r w$; Horn \& Blankson, 2012; McGrew \& Woodcock, 2001).

Carroll and the three-stratum theory. In 1993, John Carroll (1916-2003) summarized his extensive re-factor-analyses of 461 datasets in his book, Human Cognitive Abilities: A Survey of Factor-Analytic Studies. Using consistent principalfactor analyses to discern the broader factors subsuming the variables in previous human cognitive abilities studies, Carroll proposed a three-stratum model with $g$ at the highest, or Stratum III, level. The Stratum II level contained eight broad abilities that were subsumed by $g$, including fluid intelligence $(\mathrm{G} f)$, crystallized intelligence $(\mathrm{Gc})$, general memory and learning (Gsm), broad visual perception (Gv), broad auditory perception $(G a)$, broad retrieval ability $(\mathrm{Gr})$, broad cognitive speediness $(G s)$, and reaction time/decision speed $(\mathrm{G} t)$. At the Stratum I level, Carroll found 69 narrow abilities that were subsumed by the Stratum II broad factors.

Carroll's three-stratum theory provided a comprehensive taxonomy of cognitive 
abilities by integrating the most salient aspects of the previous major theories of cognitive abilities: Spearman's (1904) two-factor theory encompassing the general and specific factors, $g$ and $s$, Thurstone's (1938) theory of primary mental abilities, and Horn and Cattell's extended $\mathrm{g}_{f} \mathrm{~g}_{c}$ theory (Cattell, 1943; Horn \& Blankson, 2012; Horn \& Cattell, 1982) in which narrow abilities were subsumed under their respective broad abilities.

Cattell-Horn-Carroll (CHC) theory. Following Carroll's (1993) seminal work, McGrew (1997) analyzed the standardization sample of the Woodcock-Johnson Psychoeducational Battery - Revised (WJ-R; Woodcock \& Johnson, 1989) to resolve differences between the Horn-Cattell and Carroll models. The WJ-R, a cognitive battery structured on Horn and Cattell's $(1996,1982)$ extended $g_{f}-g_{c}$ theory (Alfonso, Flanagan, \& Radwan, 2005), was the first individually administered, standardized battery linking modern psychometric theory to clinical cognitive assessment (Schneider \& McGrew, 2012). McGrew's solutions from his factor analyses ultimately yielded the Cattell-HornCarroll (CHC) theory of cognitive abilities and included keeping quantitative knowledge $(\mathrm{G} q)$ distinct from fluid reasoning $(\mathrm{G} f)$, maintaining a broad reading and writing knowledge (Grw) factor, subsuming the narrow factor of phonological awareness under the broad auditory processing $(\mathrm{G} a)$ factor, keeping short-term memory under a broad working memory (Gsm) ability, and placing the narrow ability of associative memory under the broad factor of long-term storage and retrieval (Glr) - all issues of inconsistencies between the two theories (McGrew, 1997). Otherwise, McGrew kept Carroll's broad and narrow ability factors as Carroll $(1993,2012)$ had outlined them.

McGrew noted that, for his analysis, he did not attempt to resolve the existence of psychometric $g$ which existed in Carroll's model but not in the Horn-Cattell extended $g_{f}$ - 
$g_{c}$ theory. McGrew (2005) later described how several subsequent studies (e.g., Bickley, Keith, \& Wolfe, 1995; McGrew \& Woodcock, 2001; Taub \& McGrew, 2004) supported a three-stratum model that is a true amalgamation of the Horn-Cattell extended $g_{f}-g_{c}$ and Carroll three-stratum models. Further, results from these reviews opened the door to possible future expansion and elaboration of the theory (Schneider \& McGrew, 2012). McGrew (2005) reflected that the first published definition of "CHC theory" occurred in the WJ III technical manual (McGrew \& Woodcock, 2001, Section F5, Table 8.1), but that the term was originally coined during a 1999 private meeting between Woodcock, Gale H. Roid (the author of the Stanford-Binet Intelligence Scales, Fifth Edition; SB-5; Roid, 2003), Riverside Publishing staff, Horn, and Carroll. According to McGrew (2005), the members of the meeting agreed that "the phrase 'Cattell-HornCarroll theory of cognitive abilities' made significant practical sense, and appropriately recognized the historical order of scholarly contribution of the three primary contributors" (p. 149).

Cattell-Horn-Carroll (CHC) theory provides a comprehensive nomenclature with which to discuss the structure of the intelligence batteries analyzed in this study (McGrew, 1997, 2005; Schneider \& McGrew, 2012), specifically, the WJ III COG (Schrank, 2005; Schrank \& Wendling, 2012), the WJ III ACH (Schrank \& Wendling, 2012), the WAIS-III (see Golay \& Lecerf, 2011, for a factor analysis fitting the French WAIS-III to CHC structure), and WAIS-IV (see Benson, Hulac, \& Kranzler, 2010, for a factor analysis fitting the WAIS-IV to CHC structure). Schneider and McGrew (2012) recently provided updated definitions of the CHC taxonomy (CHC 2.0, see Schneider \& McGrew, 2012) based on their recent reviews of the current literature and reexamination 
of Carroll's (1993) work. The following definitions of CHC $g$, broad, and narrow factors reflect CHC theory as defined by Schneider and McGrew (2012), but within the limitations imposed by the CHC framework of the tests offered by the WJ III (Schrank, 2012), the WAIS-III (Golay \& LeCerf, 2011), and WAIS-IV (Benson, Hulac, \& Kranzler, 2010) as described by the most recent analytical studies of those instruments:

- General Intelligence ( $g$ ) - In CHC, $g$ represents the unitary cause of the positive manifold of the tests within the battery, as envisioned by Carroll (1993).

Schneider and McGrew (2012) encouraged readers to ignore the construct if they reject the idea of $g$.

- Fluid Reasoning (Gf) - G $f$ is defined as the ability to solve unfamiliar problems. Narrow abilities subsumed under $\mathrm{G} f$ include induction (I), defined as the ability to reason from the specific case to make inferences about the general case; deductive or sequential reasoning $(\mathrm{RG})$, defined as the ability to reason from the general case to make inferences about a specific case; and quantitative reasoning (RQ), defined as the ability to use inductive or deductive reasoning with numbers, operators, and symbols. Schneider and McGrew (2012) posited that $\mathrm{G} f$ is the broad ability most highly correlated with $g$ and that inductive reasoning is at the heart of $G f$.

- Working Memory Capacity (Gsm) - Gsm is defined as the ability to encode, hold, and operate on information in awareness. Narrow factors subsumed under Gsm include memory span (MS), defined as the ability to encode, hold, and quickly recall information in the same sequence in which it was given; and working memory capacity (MW), defined as the ability to encode, hold and operate on 
information, even in the event of potential distraction.

- Long-term Storage and Retrieval (Glr) - Glr is defined as the capacity to encode and store information, and then to retrieve it at a later time than is possible to be recalled using Gsm. No narrow factors are measured by more than one subtest in the WJ III or Wechsler scales; therefore, the Glr narrow factors are not discussed here. Only the broad factor of $\mathrm{G} l r$ was analyzed for this study.

- Processing speed (Gs) - Gs is defined as the ability to perform quickly and easily cognitive tasks that are easy enough or have been practiced enough to have become virtually automatic. The narrow ability subsumed under Gs that is measured by at least two tests in this study is perceptual speed $(\mathrm{P})$, defined as the speed with which once can compare relatively simple stimuli for similarities or differences. Schneider and McGrew (2012) suggested that P is at the core of Gs.

- Comprehension-Knowledge $(G c)-\mathrm{Gc}$ is an expression of the extent of one's skills and knowledge that have been acquired by culture and education. Narrow abilities subsumed by $\mathrm{G} c$ and tested by the batteries in this study include general verbal information (KO), defined as the depth and breadth of knowledge considered in the culture important for everyone to know, and lexical knowledge (VL), defined as the extent to which one understands word definitions. Schneider and McGrew (2012) noted that another "narrow" ability, language development, actually seems to be an intermediary skill that encompasses all language abilities working in concert. Although language development may be represented by tests in this study's batteries, its all-encompassing nature makes it difficult to determine its specific effects relative to performance (see Schrank \& Wendling, 
2012 and Flanagan, Alfonso, \& Ortiz, 2012 for differing narrow ability assignments to cognitive tests measuring $\mathrm{Gc}$ ).

-Visual-Spatial Processing $(G v)-\mathrm{G} v$ is defined as the ability to process simulated or imagined images to solve problems. The narrow abilities subsumed under $\mathrm{G} v$ and assessed by this study's batteries include visualization (Vz), defined as the ability to imagine how perceived patterns would look if changed or rotated in space, and visual memory (MV), defined as the ability to encode, store, and quickly recall complex images. Schneider and McGrew (2012) suggested that every cognitive test that measures $\mathrm{G} v$ should include at least one visualization test.

- Auditory Processing ( $\mathrm{Ga})-\mathrm{G} a$ is defined as the ability to notice and process meaningful information in auditory stimuli. In this study's test batteries, $\mathrm{G} a$ was assessed only through one of its narrow abilities, phonetic coding (PC), defined as the ability to process distinct speech phonemes. $\mathrm{PC}$ is also known as phonemic or phonological awareness, or phonological processing.

- Reading and Writing Knowledge (Grw) - Grw is defined as the extent of knowledge related to written language. Although there are several narrow factors subsumed under Grw, including reading decoding (RD), reading comprehension (RC), reading speed (RS), spelling ability (SG), English usage (EU), writing ability (WA), and writing speed (WS), the current study employed only one or fewer tests measuring each of these abilities; therefore, they were analyzed simply as part of the broad Grw factor. Most Grw tests are administered as part of an achievement test battery; indeed, the Grw tests that were analyzed in this study 
are part of the WJ III ACH battery.

- Quantitative Knowledge $(G q)-\mathrm{G} q$ is defined as the extent of knowledge related to mathematics, including acquired knowledge about mathematical concepts such as symbols, operations, computing procedures, and other mathematical skills such as calculator use. As with reading and writing knowledge (Grw), the mathematics tests presented by this study were analyzed only under the broad $\mathrm{G} q$ factor and not by their respective narrow abilities, which include mathematical knowledge (KM) and mathematical achievement (A3). Also similarly to $\mathrm{Grw}, \mathrm{G} q$ tests are administered typically as part of an achievement battery. Most of the $\mathrm{G} q$ tests in this study come from the WJ III ACH; the WAIS-III/IV Arithmetic test, however, was also analyzed as a $\mathrm{G} q$ measure.

Broad abilities explained by CHC theory but not assessed by this study's batteries include Reaction and Decision Speed (Gt), Psychomotor Speed (Gps), Domain-Specific Knowledge (Gkn), Olfactory Abilities (Go), Tactile Abilities (Gh), Kinesthetic Abilities (Gk), and Psychomotor Abilities (Gp; Schneider \& McGrew, 2012). Because these broad abilities are currently not generally included as part of an assessment battery and have not been included as part of the WJ III or WAIS-III/IV, they will not be discussed further. Please see Schneider and McGrew (2012) for a complete review of these CHC broad abilities.

CHC and the current study's test development. As the WJ-R (Woodcock \& Johnson, 1989) was structured to reflect the Horn-Cattell extended $g_{f}-g_{c}$ theory (Alfonso et al., 2005), the WJ III was revised from the WJ-R specifically to reflect the most up-todate CHC theory of its time (Schrank, 2005; Schrank \& Wendling, 2012; Woodcock et 
al., 2001, 2007). Each test of the WJ III is designed to measure one or more narrow cognitive abilities as defined by $\mathrm{CHC}$ theory and can be considered a measure of at least one broad ability (Schrank, 2005; Schrank \& Wendling, 2012).

Even the original Wechsler batteries, which were originally developed atheoretically, have been revised to embrace CHC theory (Drozdick, Wahlstrom, Zhu, \& Weiss, 2012; Keith \& Reynolds, 2010; Wechsler, 2008; Zhu \& Weiss, 2005). Little mention was made of CHC theory in Zhu and Weiss' (2005) description of the WAIS-III. Noting that Wechsler was "more of a clinician and test developer than a theorist" (p. 297), Zhu and Weiss nevertheless named the theories of Spearman and Thorndike as strong influences in Wechsler's development of the WAIS-III. With its verbal and performance scales, the WAIS-III also pays homage to Vernon's verbal/mechanical factor structure (Wasserman, 2012). Despite the predominantly atheoretical underpinnings of the WAIS-III, however, one factor-analytic study discovered that CHCderived factor structure fits the data derived from the French WAIS-III standardization sample better than does the four-factor structure designed by the test developers (Golay \& Lecerf, 2011).

Drozdick and colleagues (2012) reported that the WAIS-IV's revisions bring it into closer alignment with $\mathrm{CHC}$ theory than the WAIS-III. Specifically, test developers changed the Perceptual Organization Index (POI) to the Perceptual Reasoning Index (PRI) and added the fluid reasoning (Gf) subtest Figure Weights to honor empirical support for the importance of fluid reasoning. The developers also added Digit-SpanSequencing to the Digit Span subtest, providing another measure of working memory to support the evidence regarding the importance of the broad ability of working memory 
capacity (Gsm) to cognitive functioning. Additionally, CHC theory's support for processing speed is honored in the WAIS-IV by including the broad processing speed factor (Gs) subtests Coding and Symbol Search in the calculation of the FSIQ (Drozdick et al., 2012). Enhancing the perception of the WJ III and Wechsler scales as valid CHC measures, Flanagan and colleagues embarked on extensive cross-battery assessment research, providing a systematic and valid interpretation method for subtests across batteries based on CHC theory (Alfonso et al, 2005; Flanagan et al., 2012; Flanagan, Fiorello, \& Ortiz, 2010; McGrew \& Flanagan, 1998).

\section{Cognitive-Achievement Relations and Factor Structures}

The development of $\mathrm{CHC}$ theory has fostered a common taxonomy in shaping the discussion regarding cognitive-achievement relationships (McGrew, 2005; Schneider \& McGrew, 2012). Although debate continues regarding the relation between Spearman's (1904) $g$ (Jensen, 1998) and academic achievement, a substantial amount of research supports $g$ 's predominant role over the secondary abilities in accounting for test variance and predicting achievement (e.g., Canivez, 2011; Canivez \& Watkins, 2010; Duckworth, Quinn, \& Tsukayama, 2011; Freberg, Vandiver, Watkins, \& Canivez, 2008; Glutting, Watkins, Konold, \& McDermott, 2006; Glutting, Youngstrom, Ward, Ward, \& Hale, 1997; Johnson, Brouchard, Krueger, McGue, \& Gottesman, 2004; Kotz, Watkins, \& McDermott, 2008; Maller \& McDermott, 1997; McDermott, Fantuzzo, Glutting, Watkins, \& Baggaley, 1992; Oh, Glutting, Watkins, Youngstrom, \& McDermott, 2004; Rohde \& Thompson, 2006; Spinks et al., 2007; Watkins \& Glutting, 2000; Watkins, Glutting, \& Lei, 2007). The targets of these inquiries vary in several respects. Some studies focus on primary and secondary students (i.e., Canivez, 2011; Duckworth et al., 
2011; Freberg, 2008; Glutting et al., 2006; Kotz et al., 2008; McDermott et al., 1992; Oh et al., 2004; Watkins \& Glutting, 2000), whereas others consider college students and adults (i.e., Canivez \& Watkins, 2010; Maller \& McDermott, 1997; Rohde \& Thompson, 2006; Spinks et al., 2007). Diverse cognitive batteries have also been investigated, including the Comprehensive Ability Battery (CAB; Hakstian \& Cattell, 1975; Johnson et al., 2004), the Cognitive Assessment System (CAS; Naglieri \& Das, 1997; Canivez, 2011), the Differential Ability Scales (DAS; Kotz et al., 2008), the Hawaii Battery (HB; DeFries et al., 1974), Raven's Progressive Matrices (Raven, 1941; Johnson et al., 2004), the Mill Hill Vocabulary Scales and Raven's Advanced Progressive Matrices (Raven, Raven, \& Court, 1998; Rohde \& Thompson, 2006), the WAIS (Wechsler, 1955; Johnson et al., 2004), WAIS-Revised (WAIS-R; Wechsler, 1981; Wechsler, Maller \& McDermott, 1997), WAIS-III (Wechsler, 1997; Spinks et al., 2007), WAIS-IV (Wechsler, 2008; Canivez \& Watkins, 2010), Wechsler Abbreviated Scale of Intelligence (WASI; Psychological Corporation, 1999; Duckworth et al., 2011), Wechsler Intelligence Scale for Children-Revised (WISC-R; Wechsler, 1974; McDermott et al., 1992), WISC-Third Edition (WISC-III; Wechsler, 1991; Freberg et al., 2008; Glutting et al., 1997; Oh et al., 2004; Watkins \& Glutting, 2000; Watkins et al., 2007), and WISC-Fourth Edition (WISC-IV; Wechsler, 2003; Glutting et al., 2006; Watkins et al., 2007). Finally, different methods of analysis have been employed, including cluster analysis, multivariate analyses of variance (MANOVA) and multivariate analyses of covariance (MANCOVA; Maller \& McDermott, 1997); exploratory factor analysis (EFA) with Schmid-Leiman (1957) procedure (Canivez, 2011; Canivez \& Watkins, 2010; Johnson et al., 2004); hierarchical multiple regression (Freberg et al., 2008; Glutting et al., 1997; Kotz et al., 
2008; McDermott et al., 1997; Rhode \& Thompson, 2006; Watkins \& Glutting, 2000); linear regression (Spinks et al., 2007); and structural equation modeling (Duckworth et al., 2011; Glutting et al., 2006; Johnson et al., 2004; Oh et al., 2004). These studies all conclude, with some minor caveats, that the additional variances provided by the secondary ability factors are not enough to support either their usefulness or the extra work that would be required to interpret them as achievement predictors. In summary, this body of literature suggests that psychometric $g$ should be the principle metric by which clinicians should assess cognitive performance and predict academic achievement.

Besides this literature's implication that $g$ accounts for the major portion of total and common variance in the cognitive tests studied, some other interesting findings bear mentioning. An examination of correlations among three diverse test batteries (i.e., the Comprehensive Ability Battery , Hawaii Battery, and WAIS) determined almost perfect correlations among the three global factors $(.99, .99$, and 1.00), supporting the existence of an overarching, higher-order global factor among disparate tests (Johnson, Bouchard, Krueger, McGue, \& Gottesman, 2004). In his analysis of the Cognitive Assessment System (CAS; Canivez (2011) found that most of the total and common variance was accounted for by the global second-order factor. With the CAS's first-order factors of Planning, Attention, Simultaneous and Successive processing (PASS; Das, Naglieri, \& Kirby, 1994), however, the measure also demonstrated greater first-order variances than did other intelligence tests (see Canivez, 2011, for a review of these studies), including the WISC-IV, WAIS-IV, Stanford-Binet - Fifth Edition (SB-5; Roid, 2003), Reynolds Intellectual Assessment Scales (RIAS; Reynolds \& Kamphaus, 2003), Wechsler Abbreviated Scale of Intelligence (WASI), and the Wide Range Intelligence Test (WRIT; 
Glutting, Adams, \& Sheslow, 2000). Canivez surmised that because the tests in the CAS were specifically designed to measure PASS dimensions, they generally had lower $g$ loadings than tests in the other measures that are designed to measure more traditional and more highly g-loaded cognitive dimensions.

The predictive ability of the global-ability score over the broad factor index scores has been supported in studies measuring cognitive-achievement relations in students with widely varying broad factor index scores (Freberg et al., 2008; Kotz et al., 2008; Maller \& McDermott, 1997; McDermott et al., 1992). A longitudinal analysis of 6- to13-yearold students assessed for special education eligibility indicated that despite significant variability in first-order factor scores, the full-scale IQ (FSIQ) of the WISC-III successfully predicted future reading and mathematics achievement (Freberg et al., 2008). Refuting the idea that only flat cognitive profiles are valid, and that having a highly variable cognitive profile has less predictability (Fiorello, Hale, Holdnack, Kavanagh, Terrell, \& Long, 2007; Flanagan \& Mascolo, 2005; Kaufman, 1994), neither evaluees' profile variability status nor the interaction between profile variability status and IQ significantly predicted future achievement. Maller and McDermott (1997) produced similar results in a study with the WAIS-R and college students. Another longitudinal study examining the influence of IQ and self-control on future achievement test scores and report card grades also demonstrated the global IQ score's ability to predict future achievement test scores in children (Duckworth, Quinn, \& Tsukayama, 2011). One study demonstrating a strong correlation between mid-life FSIQ and earlier school achievement as measured by the Iowa Tests of Basic Skills (ITBS; Hoover et al., 2003) yielded evidence that the IQ-achievement relation is a remarkably stable construct 
that holds up over time (Spinks et al., 2007).

Canivez and Watkins (2010) determined with hierarchical exploratory factor analyses with Schmid-Leiman transformations (Schmid \& Leiman, 1957) that WAIS-IV subtests loaded appropriately on the manual-proposed first-order factors (Wechsler, 2008), and that these loadings accounted for more variance than did their respective WISC-IV counterparts. After finding that the second-order general factor accounted for the greatest amount of the common and total variance, however, Canivez and Watkins recommended that only the full-scale IQ (FSIQ) be interpreted until future research could bolster the claim that incremental first-order variances were significant enough to differentiate diagnosis. Two additional studies of the WISC-III's and WISC-IV's abilities to predict achievement also found that statistically significant incremental firstorder factor variances over the FSIQ score had no corresponding effects on achievement (Glutting, Watkins, Konold, and McDermott, 2006; Glutting, Youngstrom, Ward, Ward, \& Hale, 1997). These findings again prompted recommendations to interpret only the FSIQ for predictive purposes. Following up on Glutting and colleagues' (1997) WISCIII study, Oh and colleagues (2004) reiterated the suggestion to "heed the law of parsimony" (p. 169) and interpret mainly the FSIQ. They recommended, however, that one should also interpret the WISC-III factors Verbal Comprehension for reading achievement and Freedom from Distractibility for mathematics achievement because they significantly add variances that influence achievement. Other interesting findings regarding these measures include the WISC-III's consistent FSIQ-achievement associations between children referred and not referred for evaluation (Glutting et al., 1997) and the WISC-IV FSIQ's consistent prediction of academic achievement across 
diverse demographic groups (Konold \& Canivez, 2010).

On the other side of the cognitive-achievement debate spectrum, many studies have supported the importance of examining more closely the various lower-order cognitive abilities that are posited to affect academic achievement (e.g., Abu-Hamour, Al Hmouz, Mattar, \& Muhaidat, 2012; Benson, 2008; Bone, Cirino, Morris, \& Morris, 2002; Cirino, Morris, \& Morris, 2002; Fiorello et al., 2007; Flanagan \& Mascolo, 2005; Floyd, McGrew, Barry, Rafael, \& Rogers, 2009; Floyd, McGrew, \& Evans, 2008; Geary, 1993; Gropper \& Tannock 2009; Hale, Dumont, Rackley, \& Elliott, 2008; McGrew, Flanagan, Keith, \& Vanderwood, 1997; McGrew \& Wendling, 2010; Osmon, Braun, \& Plambeck, 2005; Osmon, Smerz, Braun, \& Plambeck, 2006; Parkin \& Beaujean, 2012; Proctor, 2012; Proctor, Floyd, \& Shaver, 2005; Taub, Floyd, Keith, \& McGrew, 2008; Trainin \& Swanson, 2005; Vock, Preckel, \& Holling, 2011). These studies examined the significant first-order factor loadings and predictive effects of cognitive tests with respect to the broad ability factors proposed by Carroll and his three-stratum theory, Cattell's $g_{f}-g_{c}$ theory, current $\mathrm{CHC}$ theory, or with respect to the factors suggested by the individual test manufacturers (e.g., Wechsler, 1955, 1974, 1981, 1991, 1997, 2003, 2008).

In their conclusions from a review of the literature on assessment of adults for learning disabilities, Gregg, Coleman, Davis, Lindstrom, and Hartwig (2006) reported that postsecondary evaluations occur primarily to provide students with documentation to access accommodations. The reviewers concluded that accommodations best serve students if they arise from detailed analyses of individual strengths and weaknesses profiles. This finding is shared by proponents of the cross-battery assessment approach, who posit that appropriate interventions and accommodations will most likely be found 
via a thorough understanding of normative strengths and weaknesses (Fiorello et al, 2007; Flanagan, Alfonso, \& Ortiz, 2012).

Methodological errors have been implicated in the exploration of the cognitiveachievement relations of specific factors, however. For example, Hall, Fiorello, Dumont, Will, Rackley, \& Elliott (2008) used commonality analysis to determine that subcomponent scores better predicted mathematics achievement than the global ability score did on the DAS-II. Schneider's (2008) analysis, however, demonstrated that commonality analysis is a flawed way to partition factor variance. Results from McGrew, Flanagan, Keith, \& Vanderwood's (1997) review offered insights into how specific abilities could validly be explored: Use a battery that assesses the greatest number of abilities or use a cross-battery approach that will facilitate examination of the $\mathrm{G} f-\mathrm{G} c$ abilities, become more focused on the specific assessments related to the given referral question, and do not focus on individual subtests for interpretation but rather on the common variance shared by specific abilities that create opportunities for cluster interpretation according to prevailing intelligence theory. In 1997, McGrew and colleagues had little empirical research to back up their recommendations, and they indicated such in their review. Since then, however, studies including those noted above have supported McGrew and colleagues' recommendations for the study of specific cognitive-achievement relations.

Floyd, Shands, Fawiziya, Bergeron, and McGrew (2009) confirmed the dependability of general factor loadings attributable to test characteristics in a broad variety of test batteries administered to college students. Further, both global and specific variances of the CHC broad factors were explored in a study by Floyd, McGrew, 
Barry, Rafael, and Rogers (2009). In their sample's 14 to 19 and 20 to 39 age groups, comprehension-knowledge ( $\mathrm{G} c)$, long-term storage and retrieval (Glr), and fluid reasoning $(\mathrm{G} f)$ loaded primarily on the general factor and showed higher $g$ loadings than specificity effects. Visual-spatial processing $(\mathrm{G} v)$, auditory processing $(G a)$, and processing speed (Gs) demonstrated primarily specific effects and lower $g$ loadings. Although working-memory capacity (Gsm) measured primarily specific abilities in these age groups, it showed more variability in its g-loadings across other ages. The researchers concluded that their results support CHC theory's promotion of a general factor and more specific and independent broad abilities.

McGrew and Wendling's (2010) meta-analysis of 134 analyses over the last 20 years of cognitive-achievement relations compiled much of the extant literature's findings on the influence of specific $\mathrm{CHC}$ broad and narrow factors across the primary and secondary school ages in basic reading skills (decoding and word recognition skills), reading comprehension (gaining meaning from text), basic mathematics skills (arithmetic and computation skills), and mathematics reasoning (mathematical problem solving skills). Noting that "the primary action is at the narrow ability level" (p. 669), McGrew and Wendling classified findings into "consistency of significance: high ( $\geq 80 \%$ of studies that found significant findings), medium (50\%-79\%), low (30\%-49\%), or tentative/speculative (20-29\%, based on small number of studies, or based on McGrew's exploratory multiple regressions analysis"; p. 659).

In the 14 to19 year-old age group (the highest age group for which analyses were completed), the broad abilities comprehension knowledge ( $\mathrm{Gc}$ ) and working-memory capacity (Gsm) displayed high consistency in predicting basic reading skills. The CHC 
narrow abilities predicting basic reading skills include phonetic coding ( $\mathrm{G} a-\mathrm{PC})$, memory span (Gsm-MS), and working memory (Gsm-MW; medium consistency) Knowledge ( $\mathrm{Gc}-\mathrm{KO}$; medium consistency that increases with age), and perceptual speed (Gs-P; low consistency). The consistent reading comprehension broad abilities include comprehension knowledge ( $\mathrm{G} c$; high consistency) and fluid reasoning ( $\mathrm{G} f$; tentative consistency at higher levels, possibly partially explained by narrow abilities). The reading comprehension narrow abilities include working memory (Gsm-MW), knowledge (Gc-KO), listening ability (Gc-LS), and meaningful memory (Glr-MM; high consistency), memory span (Gsm-MS; medium consistency), and phonetic coding ( $\mathrm{G} a$ PC), naming facility (Glr-NA), and perceptual speed (Gs-P; low consistency).

For basic mathematical skills, the broad abilities comprehension knowledge $(\mathrm{Gc})$, fluid reasoning $(\mathrm{G} f)$, and processing speed (Gs) display medium consistency. Basic mathematics skills narrow abilities include working memory (Gsm-MW) and processing speed (Gs-P) [high - Gs-P may be due to number facility $(\mathrm{G} s-\mathrm{N})$ and phonetic coding ( $\mathrm{G} a$-PC; tentative/speculative)]. Reflecting that achievement in mathematics reasoning will depend, at least partially, on basic mathematical skills proficiency, McGrew and Wendling listed the mathematical reasoning broad abilities for mathematical reasoning at ages 14 to 19: Gc (high consistency), G $f$ (medium consistency), and Gsm (low consistency). Narrow mathematical reasoning abilities for this age group include GsmMW (high consistency), Gs-P (medium consistency), and Ga-PC (low consistency).

McGrew and Wendling speculated that specification error, such as visual-spatial processing $(\mathrm{G} v)$ tests in current batteries not measuring the specific abilities that need to be tapped for reading or mathematics, might be one reason why Gv did not show any 
significance. They noted, however, that visual-spatial memory (Gv-MV) displayed tentative consistency in their review.

Benson (2008) used structural equation modeling with the WJ III standardization sample to determine that $g$, by affecting word reading skill development, strongly influences reading achievement until the sixth grade. Thereafter, $g$ indirectly fluency and comprehension through comprehension knowledge $(\mathrm{Gc})$ and working-memory capacity (Gsm), as reading strategies improve and students increase their verbal knowledge (Gc), and reading material becomes longer and more complex, activating Gsm. Benson's analyses also supported Gs's effects on fluency that increase with age. Taub and colleagues (2008) performed similar analyses to determine that $g$ indirectly affects, and fluid reasoning $(\mathrm{G} f)$, comprehension-knowledge $(\mathrm{Gc})$, and processing speed (Gs) directly affect, mathematical achievement.

Four $\mathrm{CHC}$ broad abilities are implicated in a study on the basic writing skills and written expression achievement of students aged 7 to 18 (Floyd, McGrew, \& Evans, 2008). In their analysis, Floyd and colleagues operationally defined standardized regression coefficients of 0.10 to be practically significant. Their results indicated that comprehension-knowledge $(\mathrm{Gc})$ was consistently the strongest predictor for basic writing skills (standardized regression coefficient of approximately 0.4 ) and written expression (standardized regression coefficient of approximately 0.32 ) for adolescents. Floyd and colleagues surmised that Gc's enhancement of basic writing skills stems from a robust vocabulary and knowledge of the world, and written expression improves with strong verbal reasoning and ability.

Floyd and colleagues (2008) posited that processing speed allows the 
automatization of basic skills to liberate cognitive resources for more complex tasks in basic writing skills. In their simultaneous multiple regression analyses, Floyd and colleagues determined that processing speed moderately predicts basic writing skills until age 17, when it declines, but strongly predicts written expression throughout the school years. Working-memory capacity, which manages conscious verbal information and writing strategy resources simultaneously, is a moderate predictor for both basic writing skills and written expression. Auditory processing, specifically phonetic coding, moderately predicts basic writing skills from 16-17 and written expression from 15-17. Long-term memory and storage, because of the early need to retrieve capitalization, punctuation, and spelling rules, declined in the prediction of basic writing skills from exerting a strong effect at age 7 to having negligible effects by adolescence. Its effects on written expression were similarly negligible. Fluid reasoning and visual-spatial processing also exerted negligible effects on both basic writing skills and written expression.

Of studies that included college students, several involved investigations of mathematical difficulties (e.g., Cirino et al., 2002; Osmon et al., 2006; Proctor, 2012). Examining Geary's (1993) theoretical model of mathematical skill, Cirino and colleagues (2002) found that the two latent domains of "semantic retrieval" and "executiveprocedural" accounted for $17 \%$ of the variance in calculation skills in undergraduate students being assessed for learning disabilities. Geary's third domain, visuospatial ability, was not found to contribute significant incremental variance to calculation skills in these students, echoing McGrew and Wendling's (2010) finding regarding visualspatial processing $(\mathrm{Gv})$. Osmon and colleagues (2006), however, explored the specific 
cognitive abilities implicated in college-age mathematics disabilities and determined that auditory processing, visual-spatial processing, and fluid reasoning directly affect mathematics processes, and long-term storage and retrieval, working-memory capacity, and comprehension-knowledge indirectly affect mathematics processes through $g$. Proctor (2012) found support through multiple regression analyses that mathematical calculation scores are influenced primarily by processing speed and working-memory capacity, and mathematical reasoning scores are influenced by comprehensionknowledge, fluid reasoning, and working-memory capacity. She acknowledged, however, that more work in the realm of narrow abilities is needed to clarify these relations.

Regarding reading problems in college students, Bone and colleagues (2002) studied undergraduate students with and without reading disabilities and found that simple IQ-achievement discrepancy interpretation did not adequately differentiate struggling readers. The narrow ability of phonetic coding ( $\mathrm{G} a-\mathrm{PA})$ differentiated students with and without reading disabilities regardless of whether they had an IQachievement discrepancy. Students who had an IQ-achievement discrepancy but did not have low reading achievement did not have PA deficits. Osmon and colleagues (2005) determined that visual-spatial processing and working-memory capacity directly influence reading ability. Processing speed and working-memory capacity, in addition to semantic processing and word reading, differentiated college students with and without learning disabilities in a study by Trainin and Swanson (2005). The authors noted, however, that achievement across groups was similar, perhaps due to the learning disabilities group's compensatory reliance on verbal abilities, metacognitive learning 
strategies, and help seeking.

Gropper and Tannock (2009) explored specific cognitive challenges in the face of ADHD. Their findings supported the hypotheses that students with ADHD have difficulties with auditory-verbal working memory and that these deficits correspond to a lower grade-point average (GPA). Adults with ADHD also exhibit difficulties with visual working-memory storage capacity (Finke et al., 2011; Gropper \& Tannock, 2009) and visual memory (Shang \& Gau, 2011), resulting in an overall "reduced general attentional capacity" (p. 897, Finke et al, 2011) and lower GPAs (Gropper \& Tannock, 2009; Kraft, 2010).

The studies discussed previously organized themselves around current $\mathrm{CHC}$ theory in which broad and narrow abilities were examined for their influence on achievement. Some studies have explored cognitive-achievement relations vis-à-vis Cattell's $(1963,1967)$ investment theory. Ferrer and McArdle (2004) examined Cattell's (1963, 1987) investment hypothesis that fluid reasoning abilities are invested in the development of comprehension-knowledge/crystallized intelligence and thereby lead to enhanced academic and other life outcomes. Although they did not find support for fluid reasoning's specific time-lagged effect on comprehension-knowledge, as would be predicted by Cattell's theory, Ferrer and McArdle did find that fluid reasoning is a leading indicator of the school achievement constructs of academic knowledge and quantitative ability, constructs Cattell (1987) also construed as measures of crystallized abilities. Ferrer and McArdle's results therefore partially supported Cattell's investment theory but suggested a more complex interplay between fluid reasoning and crystallized intelligence than Cattell had posited. Kan and colleagues' (2011) work also supported 
this finding and added that verbal comprehension may, in fact, mediate fluid reasoning's effect on the comprehension-knowledge broad factor because it predicts crystallized intelligence.

Kaufman, Reynolds, Liu, Kaufman, and McGrew (2012) examined the conorming samples of the WJ III cognitive and achievement batteries (Woodcock, McGrew, \& Mather, 2001a, 2001b), the Kaufman Assessment Battery for Children-2nd edition (KABC-II; Kaufman \& Kaufman, 2004a), and the Kaufman Test of Educational Achievement-2nd edition (KTEA-II; Kaufman \& Kaufman, 2004b) Comprehensive Form. They concluded that "Cognitive $g$," measured by the cognitive abilities tests, and "Achievement $g$," measured by the achievement tests, are highly correlated at .83 but are not unitary. Kaufman and colleagues provided additional evidence that Cognitive $g$ and Achievement $g$ are discrete constructs by noting that their correlations increase with age, and they cited Cattell's (1987) investment hypothesis as one reason why this might be the case.

In sum, cognitive-achievement relations research continues to clarify the associations among $g$, the $\mathrm{CHC}$ broad and narrow abilities, and the achievement domains they are purported to influence, but the issue is far from resolved. Hopefully, the more researchers know about how particular cognitive abilities influence achievement, the better position practitioners will be in finding ways to help struggling students. In light of the recognition that these relations have yet to be definitively determined, future research using valid methodologies should continue to shed light on this noteworthy endeavor. 


\section{The Purported Benefits of Adding Subtest Scores to a Cognitive Battery}

Clarification of the relations among cognitive abilities and academic achievement has been one important outcome of CHC theory (McGrew \& Wendling, 2010). Although there are substantial admonitions in the literature to interpret IQ scores only at the global level (Canivez, 2013; Kahana, Youngstrom, \& Glutting, 2002; Kotz et al., 2008; Nelson \& Canivez, 2012; Watkins \& Glutting, 2000, Watkins et al., 2007; Youngstrom, Kogos, \& Glutting, 1999), McGrew and Wendling concluded from their meta-analysis that "the most important focus for $\mathrm{CHC}$ cognitive-achievement relations is at the narrow ability level” (p. 669) and that future intelligence test construction efforts should be aimed at providing more "validated narrow cognitive ability indicators" (p. 669). Currently, however, intelligence tests rarely provide more than one subtest per narrow ability, which undermines valid interpretation at that level. The CHC cross-battery assessment approach (Flanagan et al., 2012; Flanagan, Fiorello, \& Ortiz, 2010; Flanagan, Ortiz, \& Alfonso, 2007) has been endorsed as a method to obtain additional information about broad and narrow ability performance by aggregating subtest scores from different intelligence batteries. Joint confirmatory factor analyses have explored the CHC broad and narrow abilities included in major intelligence tests (Keith, Kranzler, \& Flanagan, 2001; Phelps et al., 2005; Sanders, McIntosh, Dunham, Rothlisberg, \& Finch, 2007) and support combining tests from different batteries to measure the broad and narrow abilities more comprehensively.

In current test batteries, subtest scores, each typically reflecting a unique narrow ability, are combined to form a more reliable and valid composite score that purportedly measures the subsuming CHC broad ability (McGrew, 1997; Schneider, 2013). Some 
proponents of the $\mathrm{CHC}$ cross-battery approach advise not interpreting the composite score if two or more subtests in the composite differ substantially (Fiorello, Hale, \& Wycott, 2012). There are several reasons why subtest scores within a composite might differ, including unique narrow abilities being assessed for which there are specific variances that are not shared by the common factor in the composite, the subtests each loading differently on the common factor, and other moderators affecting factor loadings (Schneider, 2011). Because other moderators (e.g., evaluee fatigue or perceptions, test administrator characteristics, test administration anomalies) can affect loadings, the clinician might choose to administer an additional subtest if there is reason to think current scores might not validly reflect the evaluee's abilities in the given subtest area. Schneider (2013) highlighted, however, why variability within the composite does not singularly invalidate the composite score. Typically, subtest scores differ because of differences in specific influences and error. It is the common (and presumably constructrelevant) influence that makes the scores similar to each other. Specific influences and error are just as likely to raise a subtest score as they are to lower the subtest score. On average (over the long term), they tend to cancel each other out. Thus, the best estimate of the common (and presumably construct-relevant) influence is the average of the subtest scores. Therefore, the predictive validity of the composite is likely to remain the same regardless of the discrepancy or consistency of the subtest scores (Watkins et al., 2007). Further, following the logic of not interpreting composite scores due to subtest variability would preclude interpreting subtest scores if items within the subtest were highly variable. Schneider (2011) concluded that the suggestion to avoid composite interpretation when subtest scores are variable disregards the need to extract the true 
score variance from the error variance, which is the main reason why subtest scores are not interpreted separately.

In summary, there are legitimate reasons to supplement a core battery with additional subtests, including comprehensively measuring narrow ability constructs not adequately addressed by the core battery and testing the hypothesis that divergent scores might be attributable to non-test specific error. Some researchers who advocate a crossbattery assessment approach suggest that composite scores in which subtest scores are highly variable should not be interpreted, but should be amended with additional tests to provide incremental validity to the composite score (Fiorello et al., 2012; Flanagan et al., 2007; Flanagan et al., 2010, Flanagan et al., 2012). A statistical inquiry into withincomposite differences, however, illustrates that the composite score is likely to reflect the true score regardless of the similarity or difference of the subtest scores (Schneider, 2011). Testing this hypothesis empirically will illuminate the incremental value of additional test time and effort.

\section{Disability Service Utilization on Achievement}

The passage of federal legislation Section 504 of the Rehabilitation Act in 1973 and the Americans with Disabilities Act (ADA) in 1990 (reauthorized in 2008), which requires colleges to provide appropriate access for all students with disabilities, has greatly increased postsecondary access for students with disabilities (Cory, 2011; Hadley, 2011). Wilson, Getzel, and Brown's (2000) review of disability services at a university in the eastern United States indicated that 60 percent of students requesting services wanted support for psychological or learning disabilities. The range of psychological disabilities accommodated by university disabilities offices includes ADHD 
(approximately 2-8\% of college students; DuPaul, Weyandt, O'Dell, \& Varejao, 2009); mental health impairments, including the anxiety disorders, depressive disorder, bipolar disorder, obsessive-compulsive disorder, panic disorder, post-traumatic stress disorder (PTSD), schizophrenia, and seasonal affective disorder (SAD; between 15-30\%;

Goldman, Rye, \& Sirovatka, 1999; Eisenberg, Golberstein, \& Gollust, 2007); learning disabilities (approximately $5 \%$ of college students; Murray \& Wren, 2003; Orr \& Hammig, 2009); and high functioning autism spectrum disorder (HFASD; between .7 and $1.9 \%$ of college students; White, Ollendick, \& Gray, 2011). Recommendations to enhance disability support services and optimize the university disability resource center's performance focus on student training and advising (including help with course selection and scheduling, tutoring, mentoring, academic skills assessment, and training), self-advocacy training, disability-related counseling, disability-related support groups, accommodation support services (including scribes, readers, test aides and proctors, and classroom assistants), and robust student and service data collection to assess and track parameters such as student demographics, service utilization, achievement outcomes, and unmet demand (Wilson, Getzel, \& Brown, 2000). Stodden, Brown, and Roberts (2011) found that student outcomes are enhanced to the extent that a university disability resource office successfully negotiates and administers these varied time- and personnelintensive tasks.

Unlike elementary and high school where IDEA 2004 ensures every student with a disability shall be identified and provided accommodations or interventions to ensure a free and appropriate public education in the least restricted environment (Office of Special Education Programs, 2012), college students with suspected or diagnosed 
disabilities must initiate their own processes and identify themselves, as well as provide adequate documentation regarding their disabilities and resulting accommodations and service needs (Hadley, 2011).

Robust student mental health requires the ability to think, act, and socialize independently. These capacities are important factors as one transitions from the nurturing environment of high school where services were presented to the student, to proactively acquiring the disability support needed to achieve goals (Stodden, Whelley, Chang, \& Harding, 2001). One qualitative study indicated that successful students with disabilities share certain behavioral and emotional strategies when requesting academic accommodations, including accepting their disability, negotiating with their instructors with adroit interpersonal skills to receive accommodations that will maximize their academic potential, and downplaying their disability status to the broader college community (Barnard-Brak, Lechtenberger, \& Lan, 2010). Recognizing that much of the research on student access to disability services has been qualitative rather than quantitative, Barnard-Brak, Davis, Tate, and Sulak (2009) conducted a quantitative study to analyze the factors that would predict students' likelihood of accessing services. The researchers found that students' requests for and access to services depended on their acceptance of and attitude toward their disability, their attitude about receiving accommodations, and their belief about whether their request for accommodations would be honored in a welcoming and supportive environment. Developing the Attitudes Toward Requesting Accommodations (ATRA) rating scale to discern the nature of a college student's acceptance of his or her disability and feelings about requesting accommodations among college students with disabilities, Barnard-Brak, Sulak, Tate, \& 
Lechtenberger (2010) supported the validity of their previous studies relating student attitudes and service access. Colleges that fully embrace disability as a vital aspect of campus diversity will likely have students who perceive campus personnel as responsive to their needs, making it more probable that students will be open and willing to access services and use accommodation letters to gain needed supports (Cory, 2011).

Students' perceptions about the openness of the university to supporting students with disabilities are also shaped by their interactions with faculty and staff who vary with respect to their willingness to accommodate students (Barnard-Brak \& Lan, 2010; Murray, Lombardi, \& Wren, 2011). The range of "reasonable accommodations" depends on individual student needs and should be negotiated between faculty, student, and disability resource office personnel on an individual basis (Cory, 2011). The accommodation letter can be a good starting point from which the student can discuss specific needs with the professor (Cory, 2011). It is incumbent upon the mental health practitioners in the university disability resource office to provide competent and ethical counseling and coaching to students to help them develop skillsets that allow them to negotiate successfully for optimal accommodations (Cornish, Gorgens, Monson, Olkin, Palombi, \& Abels, 2008). Disability resource office staff can also train faculty and staff about the university's responsibility to adhere to the ADA mandate to provide appropriate access, the proper role of accommodations to enhance learning for individuals with disabilities (Murray et al., 2011), and suggestions for universal design to individualize instruction (Orr \& Hammig, 2009). Stodden and colleagues (2011) concluded from their climate assessment of the university environment for students with disabilities that professional faculty development and increasing positive interactions between faculty, 
staff, and students with disabilities are needed to improve student academic outcomes.

Getzel and Thoma (2005) conducted a qualitative study in which they convened focus groups of successful college students with disabilities, 41 percent of whom experienced learning or other psychological disabilities. One overarching theme in this group was that students' development of metacognitive and organizational strategies played a critical role in their confidence and success in self-determination. Specific skills that assist students in their development include the ability to appraise their strengths and weaknesses realistically, negotiate optimal accommodations with faculty and staff, become knowledgeable about the supports and services that are available to them, and access those supports when necessary. These skills can all be taught within a coaching/ counseling context provided by the disability resource office.

ADHD is currently estimated to affect approximately 4.4 percent of the adult population, although up to 16 percent exhibit subthreshold criteria for ADHD (Kraft, 2010). Of the adult population with ADHD, only half were diagnosed as children. DuPaul, Weyandt, O'Dell, and Varejao (2009) reported that nearly a fourth of college students with disabilities are diagnosed with ADHD, with many of them being diagnosed after they started attending college. Many of these students struggle academically (Reaser, Prevatt, Petscher, \& Proctor, 2007). A meta-analysis by Frazier, Youngstrom, Glutting and Watkins (2007) highlighted the "moderate to large discrepancy" (p. 59) in academic achievement between students with and without ADHD and noted that these results are most significant when comparing results from standardized achievement tests. This study also uncovered moderate to large effects of ADHD symptoms on GPA, indicating a more universal effect of ADHD on student achievement. Adults with ADHD 
demonstrate difficulties with planning and attentional-set shifting (McLean et al., 2004). Further, Glutting, Youngstrom, and Watkins (2005) found that ADHD symptoms, as self reported by college students, can be reliably factored into the three dimensions of inattention, hyperactivity, and impulsivity. The researchers noted that the effects of ADHD tended to decrease with age, perhaps due to students with more severe symptoms dropping out of school, because symptoms tend to subside as individuals mature into adults (Barkley, 2010), or because students learn to compensate for their difficulties by learning metacognitive strategies to check their work or obtain help when they need it (Manalo, Ede, \& Won-Toi, 2010). Nevertheless, Frazier and colleagues (2007) discovered that both student and parent ratings of student inattentiveness predicted academic probation status. Inattention also predicted poorer study skills, social adjustment, and academic achievement in a study of college ADHD samples in China and the United States (Norvalitis, Sun, \& Zhang, 2009).

Although they readily admitted that medication and behavioral training were the primary interventions suggested for children with ADHD, Goldstein and Naglieri (2008) found evidence to support the value of cognitive training and planning strategy instruction to develop metacognitive and self-regulatory skills. An important role of the disability resource office is to enhance students' self-determination skills and ultimate independence by providing coaching or counseling to increase student confidence and competence in these skills (Hadley, 2011). Indeed, students with disabilities reported in one survey that they preferred to learn how to self-advocate rather than have someone in the university do it for them (Stodden et al., 2001). In one qualitative study, students with ADHD reported positive effects from coaching (Parker \& Boutelle, 2009). 
Specifically, learning time management and organizational skills in a personalized, nonjudgmental, and self-directed endeavor helped the students reach important goals and become more self-confident and autonomous.

With 20 percent of the overall American population affected by mental health disorders (Goldman, Rye, \& Sirovatka, 1999), college students at risk for psychiatric disabilities have been estimated as high as 30 percent, with 15 percent of the students at a large Midwestern university receiving counseling or medication for a psychiatric condition in a year and another 15 percent reporting symptoms but not seeking help (Eisenberg, 2007). Fostering resilience positively influences college students with psychiatric disabilities and helps them stay in school (Hartley, 2010). Disability office counselors and coaches can help students build resilience by enhancing trust through the therapeutic alliance, facilitating resilience factors such as stress management and cognitive behavioral techniques to ameliorate anxious and depressive symptoms, and providing academic support by teaching and promoting study, time management, and help-seeking skills (Hadley, 2011; Hartley, 2010). Other supports include academic coaching related to specific course content, remedial support for cognitive challenges related to attention, working memory, planning, and problem solving (Manalo, 2010; Reaser et al., 2007), and training in relaxation and mindfulness techniques to reduce anxiety and depression affiliated with learning problems and pressure to produce (Prevatt, Welles, Juijun, \& Proctor, 2010; Zylowska \& Siegel, 2012).

With the finding that GPA is related not only to cognitive ability but also to procrastination and study avoidance in students with learning disabilities, interventions to reduce procrastination in this group are indicated (Murray \& Wren, 2003). Carter (as 
cited in Webberman, 2011) advocated the development of a structured supportive relationship of mutual accountability and integrity between coaches and students to give students an increased sense of order. Sessions are more directive in the beginning and gradually become student driven as the student develops a keener sense of autonomy and self determination. In a study including college students with ADHD and learning disabilities, academic coaching significantly improved academic outcomes (Allsop, Minskoff, \& Bolt, 2005). Measured in terms of GPA post-intervention compared to GPA pre-intervention, improvement was attributed qualitatively to the therapeutic alliance between the coach and the student and the student's ability to use learned academic strategies independently. Two factors identified as hampering achievement goals included cognitive or achievement deficits that made learning the strategies needed for improvement difficult and emotional or medication difficulties that limited attention or motivation during sessions.

White and colleagues' (2011) study of college students identified with high functioning autism suggests that many of these students are diagnosed in college but suffered from difficulties with social anxiety, depression, and aggression prior to their admittance to the university. Remarking that still little is known about how best to serve this group, White and colleagues recommended coaching strategies that ameliorate difficulties with time management, promote self-advocacy, reduce distractibility, and help resolve interpersonal problems and loneliness to facilitate these students' adjustment to university life. In short, strategies similar to those advocated for students with ADHD and learning disabilities may be attuned to enhance achievement outcomes for students with autism spectrum disorders. 
In summary, university disability offices are called upon to provide a range of supports and services for students requesting them, as mandated by the ADA. This review has demonstrated several factors related to enhanced learning outcomes, including fostering student independence and self-advocacy skills; providing coaching strategies to enhance metacognitive, time management, organizational, and self-regulation skills; counseling to manage psychiatric symptoms and enhance disability acceptance, and raising awareness and acceptance in the broader university community. Students with disabilities who use services generally show enhanced academic achievement in the form of improved GPAs, but successful service utilization may depend on cognitive ability and mental health status at the time services are rendered. Additional analyses of these relations will elucidate the importance of these variables on students' academic success.

\section{The Present Study}

The present study examined the relations among tests of cognitive ability, academic achievement, and mental health measures in a sample of referred college students. The research aim and specific hypotheses for this study were as follows:

\section{Research Aim}

Distinctive cognitive and achievement profiles can be obtained by clarifying the various abilities that were measured in this dataset. Because everyone has a unique profile and different groups have different profiles (Glutting \& Watkins, 1997; Kamphaus, Winsor, Rowe, \& Kim, 2005), profiles can be compared for individual and group differences. This report would be unable to describe every possible group available in the dataset, but I explored some of the groups for whom parameters could be reliably estimated. Analyses were provided for the cognitive and achievement profiles of 
the largest demographic groups in the dataset, as well as of the largest diagnostic groups, including students who were given no formal diagnosis (i.e., no disability was identified).

It would be an exercise in circular reasoning to compare diagnostic profiles for explanatory purposes when those same profiles were used as part of the diagnostic decision-making process. Therefore, no attempt at an explanatory account of the diagnostic conditions was made. Having good diagnostic descriptions is nevertheless valuable. Descriptions can inform diagnostic prototypes - heuristics about what a prototypical case might look like. Westen, Shedley, and Bradley (2006) found that empirical prototypes of clients with personality disorders assisted clinicians in predicting meaningful variables such as adaptive functioning and treatment response better than did diagnoses derived from the Diagnostic and Statistical Manual of Mental Disorders Fourth Edition - Text Revision (DSM-IV-TR). Similar results have recently been found with empirical prototypes of individuals with mood and anxiety disorders (DeFife et al., 2013).

There is another reason that it is useful to have good descriptions of cognitive profiles of people with certain disorders, even if the cognitive profiles were used, in part, to diagnose the disorder. Suppose that it is a shared clinical myth that people with ADHD have extremely poor auditory processing but that an auditory processing deficit, in truth, has no predictive validity in the diagnosis of ADHD. Even if auditory processing deficits are used (incorrectly) as indictors of ADHD in the diagnostic process, the average person diagnosed with ADHD will have a better auditory processing score than the shared clinical myth suggests that person would have. If the new norms influence the shared clinical myth (i.e., that an auditory processing deficit is not as strong 
an indicator as previously believed), the next descriptive study of cognitive abilities of people with ADHD will show that an auditory processing deficit is an even weaker indicator of ADHD. Provided that new descriptive studies influence practitioners' schemas about the prototypical case of ADHD, several rounds of new descriptive studies of the cognitive abilities of people with ADHD will eventually result in auditory processing being dropped as an indicator of ADHD. Thus, descriptive studies of symptoms of disorders can have a corrective influence on future diagnostic procedures.

ADHD. Descriptive comparisons of the students of this dataset may also inform treatment or accommodation options as well as future work in the field. For example, students with ADHD have a greater risk of lower academic achievement levels than students without ADHD (DuPaul, Weyandt, O’Dell, \& Varejo, 2009; Frazier, Youngstrom, Glutting, and Watkins, 2007). Further, several studies indicate that students with ADHD are more likely than their non-affected peers to have working memory, visual memory, and processing speed difficulties, but findings are inconsistent across studies (Finke et al., 2011; Gropper \& Tannock, 2009; Nigg et al., 2005; Shang \& Gau, 2011).

Learning disabilities. Some information is available on the typical profiles of students diagnosed with different types of learning disabilities (e.g., Compton, Fuchs, Fuchs, Lambert, \& Hamlett, 2012; Murray \& Wren, 2003). Mrazik, Bender, and Makovichuk (2010) found working and auditory memory deficits in college students who met the DSM-IV criteria for Reading Disorder and Disorder of Written Expression. As with the profile analyses of students with ADHD, these data will inform how study findings compare with findings from this sample. 
Emotional disorders. Regarding the cognitive profiles of students diagnosed with mood disorders, a recent literature review illustrated the fairly consistent finding that individuals with depression perform more poorly than their non-affected peers on intelligence tests due to difficulties with verbal and spatial memory and attention (Francomano, Bonanno, Fuca, La Placa, \& La Barbera, 2011). Castaneda, TuulioHenriksson, Marttunen, Suvisaari, and Lonnqvist (2008) found that individuals with depression who were not responsive to medication treatment exhibited poorer global functioning on cognitive tests than did those who were responsive to the anti-depressant medication. Profile analyses of students diagnosed with mood and anxiety disorders may additionally inform our understanding of and potential treatment options for these individuals.

Foreign language learning difficulties. Many students enter the disabilities resource center requesting an exemption from the university's foreign language requirement. After a board reviews the student's petition, "substitution" status is conferred (allowing the student to replace the language class with a culture class) depending on the extent to which the student has attempted a foreign language without success in high school or college classes, compared to his performance in other classes. The profile picture for these students, however, is unclear (Collins, 2012, personal communication). Some evidence exists that students who struggle with foreign language acquisition also exhibit deficits relative to non-affected peers on cognitive and achievement tests measuring phonetic coding and general native language ability (Carroll, 1990; Ferrari \& Palladino, 2007; Robinson, 2001; Sasaki, 2012; Sparks, Patton, Ganschow, Humbach, \& Javorsky, 2006), working memory (Andersson, 2010; Gilabert 
\& Munoz, 2010; Riesiewicz, 2008; Safar \& Komos, 2008), inductive reasoning (Carroll, 1990; Riesiewicz, 2008; Silva \& White, 1993), spelling (Scott, Bell, \& McCallum, 2009; Sparks et al., 2006), and vocabulary (Riesiewicz, 2008). Examining the cognitive and achievement profiles of students who requested a foreign language exemption will clarify an empirical prototype of this group.

\section{Hypothesis 1 - Cognitive-Achievement Tests and CHC Factor Analysis}

The Cattell-Horn-Carroll (CHC) theory of cognitive abilities is the current gold standard of our understanding of cognitive functioning (McGrew, 1997, 2005; Schneider \& McGrew, 2012). The CHC model provides a factor model structure and comprehensive nomenclature with which to discuss the structure of the intelligence batteries. Within the CHC model, cognitive abilities have been organized into three strata: the global intelligence factor, up to 10 broad factors encompassed by the global factor, and more than 70 narrow factors under the broad factors. Research is ongoing regarding the $\mathrm{CHC}$ taxonomy, however, and the conversation continues to evolve about the nature and description of cognitive abilities (Schneider \& McGrew, 2012).

The cognitive tests examined in this study are appropriate instruments for measuring cognitive abilities within the $\mathrm{CHC}$ nomenclature. Whereas the WJ III cognitive and achievement batteries were designed specifically with $\mathrm{CHC}$ theory in mind, the WAIS-III has been criticized for its lack of theoretical basis (Schrank \& Wendling, 2012; Zhu \& Weiss, 2005). Several independent studies, however, indirectly support the WAIS-III's alignment with CHC factors (see Zhu \& Weiss, 2005, for a review). With the strengthening empirical support for CHC theory, the WAIS-IV was specifically revised from the WAIS-III to measure aspects of cognitive functioning that are more aligned with 
CHC theory (Drozdick, Wahlstrom, Shu, \& Weiss, 2012). Many of the cognitive tests offered by the WJ III and the WAIS batteries, however, are not pure tests of the cognitive abilities they purport to measure (Drozdick et al., 2012; Schrank \& Wendling, 2012) and load onto more than one factor. There is also continuing discussion about how tests load onto specific narrow factors (Flanagan, Alfonso, \& Ortiz, 2012; McGrew, 2011).

I hypothesized that the results of analyses conducted with this sample would support current $\mathrm{CHC}$ theory's factor model of latent cognitive abilities. Studies have compared the model fit of the $\mathrm{CHC}$ broad factor taxonomy with the standardization samples of well-known and validated intelligence batteries, including the French WAISIII (Golay \& Cerf, 2011), the WAIS-IV (Benson, Hulac, \& Kranzler, 2010), and the WJ III (Floyd, McGrew, Barry, Rafael, and Rogers, 2009). Unlike these researchers' focus on the standardization samples, however, I examined a sample of college students with suspected learning disabilities or psychological disorders that interfere with academic functioning. Confirmatory factor analyses were used to compare the fit of this dataset's WAIS-III, WAIS-IV, and WJ III test scores to the fit of scores from the respective standardization samples in relation to the $\mathrm{CHC}$ models. The current analyses were expected to clarify the extent to which this dataset's cognitive and achievement subtest scores relate to the $\mathrm{CHC}$ factors they are purported to measure. I expected that, consistent with previous studies, the structure of cognitive abilities observed in this dataset would be comparable to that observed in the standardization samples for each of the cognitive batteries.

Figures 1 and 2 illustrate the first measurement model to be tested. Both datasets 
include cognitive tests from their respective WAIS batteries and from the WJ III Tests of Cognitive Abilities (WJ III COG). Figure 1 lists the cognitive tests from the WAIS-III database $(N=1,040)$ that was generated from 2000 to 2010, and Figure 2 indicates the tests from the WAIS-IV database $(\mathrm{N}=252)$ that was generated from 2010 through December 2012.

Several caveats regarding these models should be considered. First, these models list only the narrow factors measured by at least two tests of the current dataset even though more than $70 \mathrm{CHC}$ narrow factors have been found to support cognitive functioning (Schneider \& McGrew, 2012). Narrow factors for which only one test is available have been subsumed by their respective broad factors. Additionally, although Figures 1 and 2 represent a model well supported by some current studies (Benson, Hulac, \& Kranzler, 2010; Floyd, McGrew, Barry, Rafael, \& Rogers, 2009; Golay \& Cerf, 2011; Schrank \& Wendling, 2012), inconsistencies found in some factor loadings (e.g., Benson, Hulac, \& Kranzler, 2010; McGrew \& Wendling, 2010) suggest minor alterations to the model could and should be tested. Further, the WAIS batteries' subtest Arithmetic has been excluded from this model because it aligns more readily as an achievement measure (Phelps, McGrew, Knopik, \& Ford, 2005). 


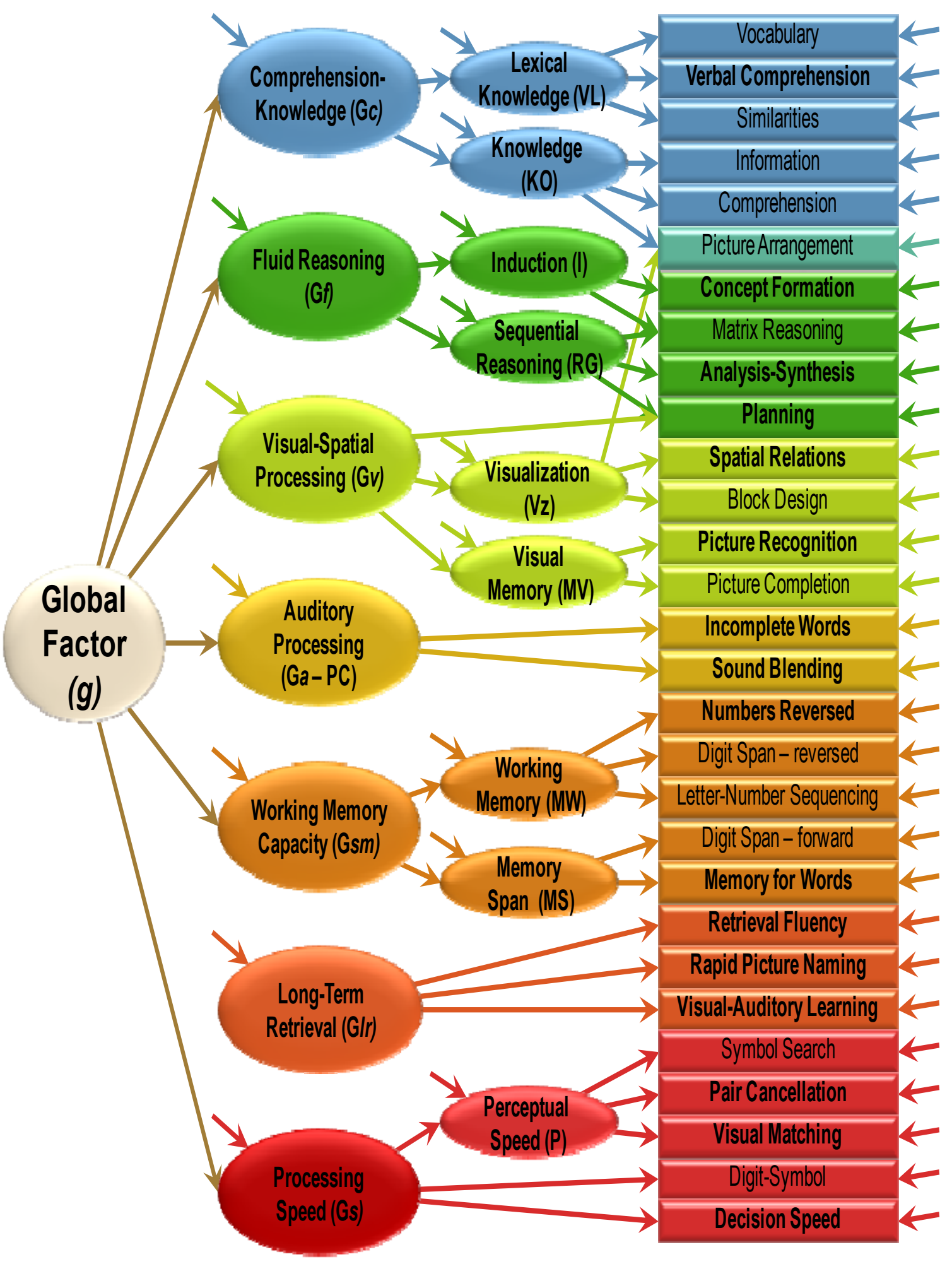

Figure 1. Measurement model of WAIS-III and WJ III COG with CHC broad factors and narrow factors measured by least two subtests. WJ III subtests are noted in bold. 


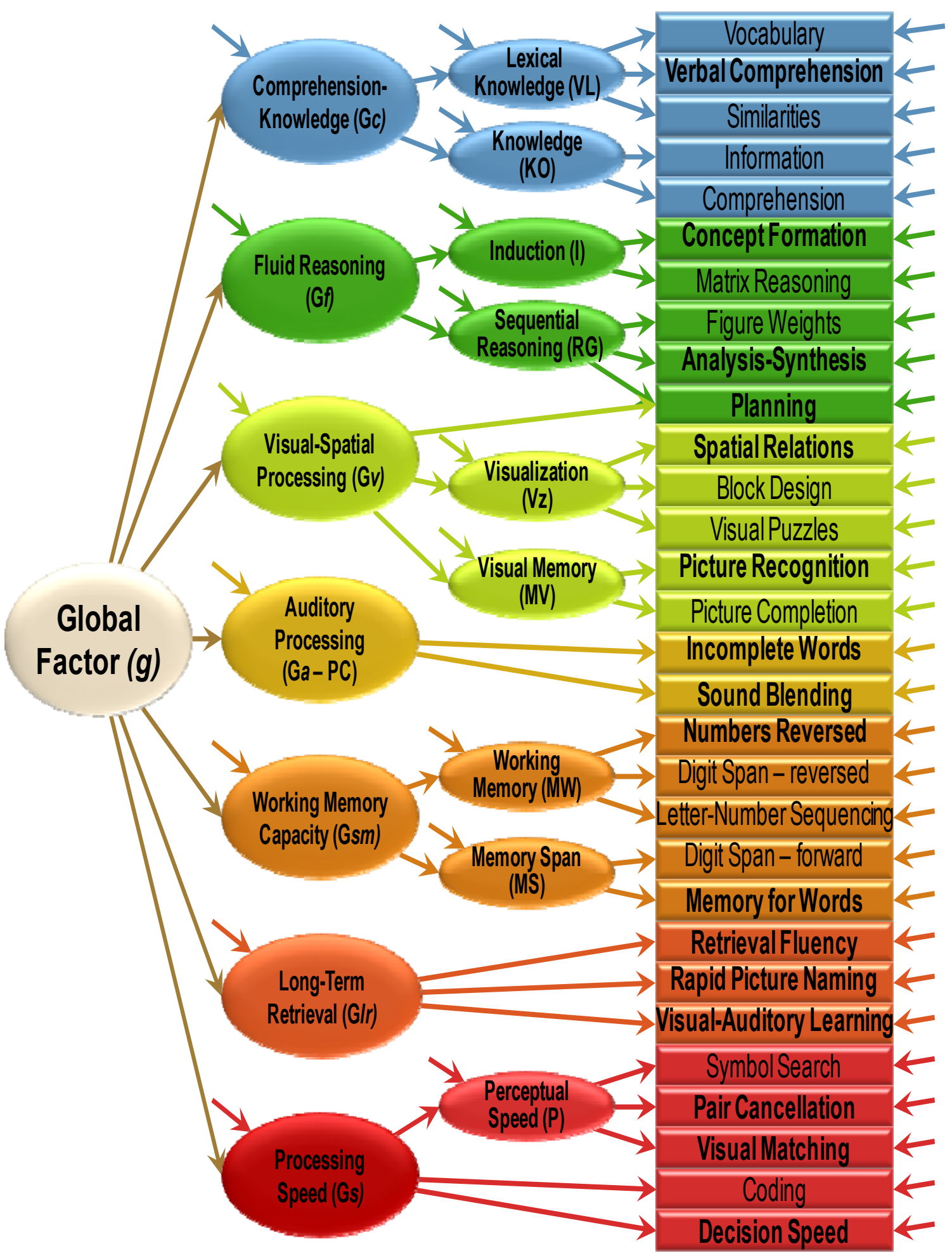

Figure 2. Measurement model of WAIS-IV and WJ III COG with CHC broad factors and narrow factors measured by least two subtests. WJ III subtests are noted in bold. 
CHC theory was founded in part on work from Cattell's (1943) work on fluid and crystallized intelligence $\left(g_{f} g_{c}\right)$ theory. Cattell originally hypothesized that $g$ was actually two separate general factors: $g_{c}$, crystallized intelligence that comprises what an individual has learned through formal education and culture, and $g_{f}$, fluid intelligence that represents a more neurologically based novel reasoning facility that is relatively independent of culture or education. Figures 3 and 4 illustrate an alternative measurement model in which the WJ III Tests of Achievement (WJ III ACH) and the WAIS Arithmetic test are considered within the broad $\mathrm{CHC}$ factors of reading and writing ability $(\mathrm{Grw})$ and quantitative reasoning $(\mathrm{G} q)$ in a two primary-factor model reminiscent of Cattell's work. 


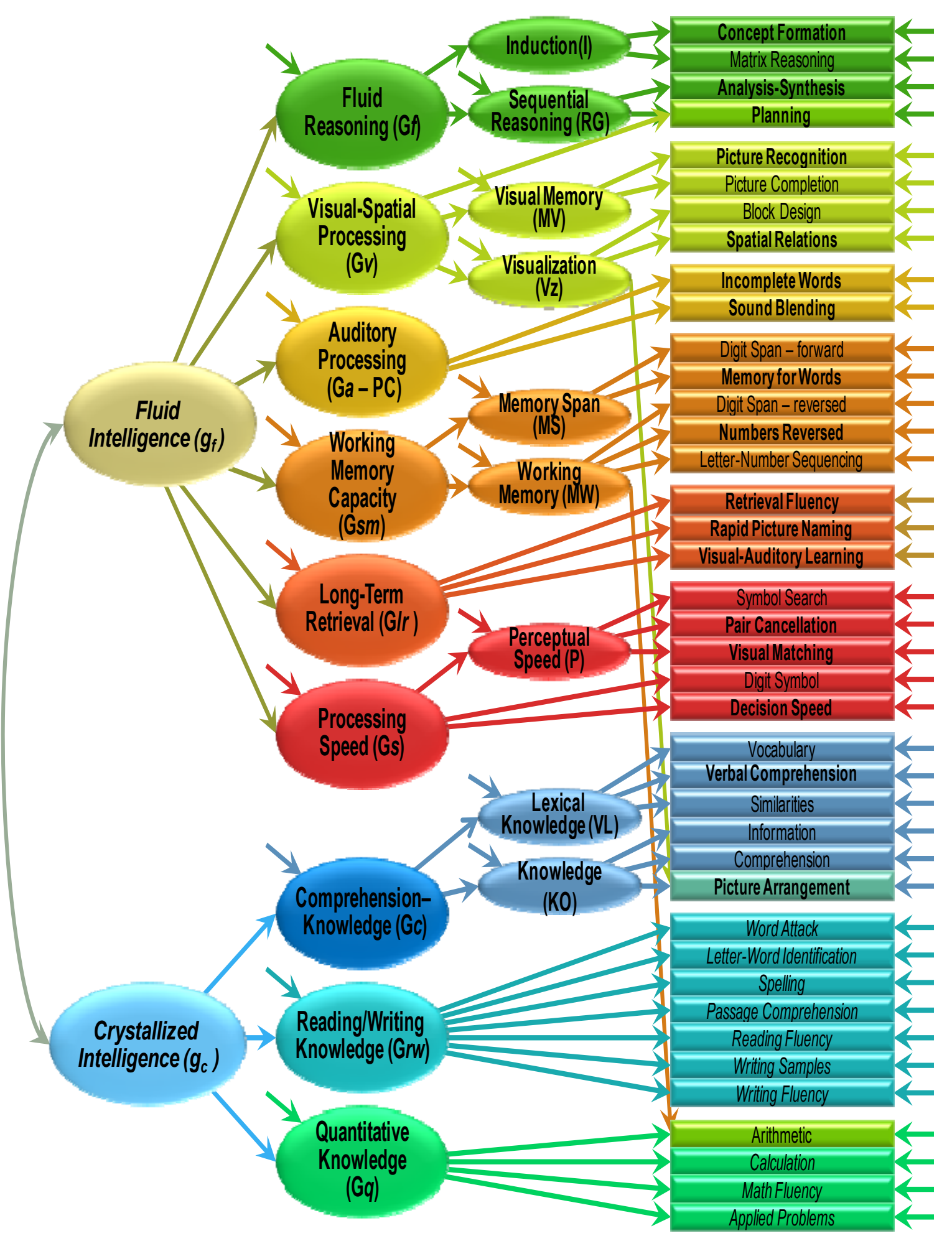

Figure 3. WAIS-III, WJ III COG, and WJ III ACH in $g_{f} g_{c}$ measurement model. WJ III COG tests are in bold; WJ III ACH tests are in italics. 


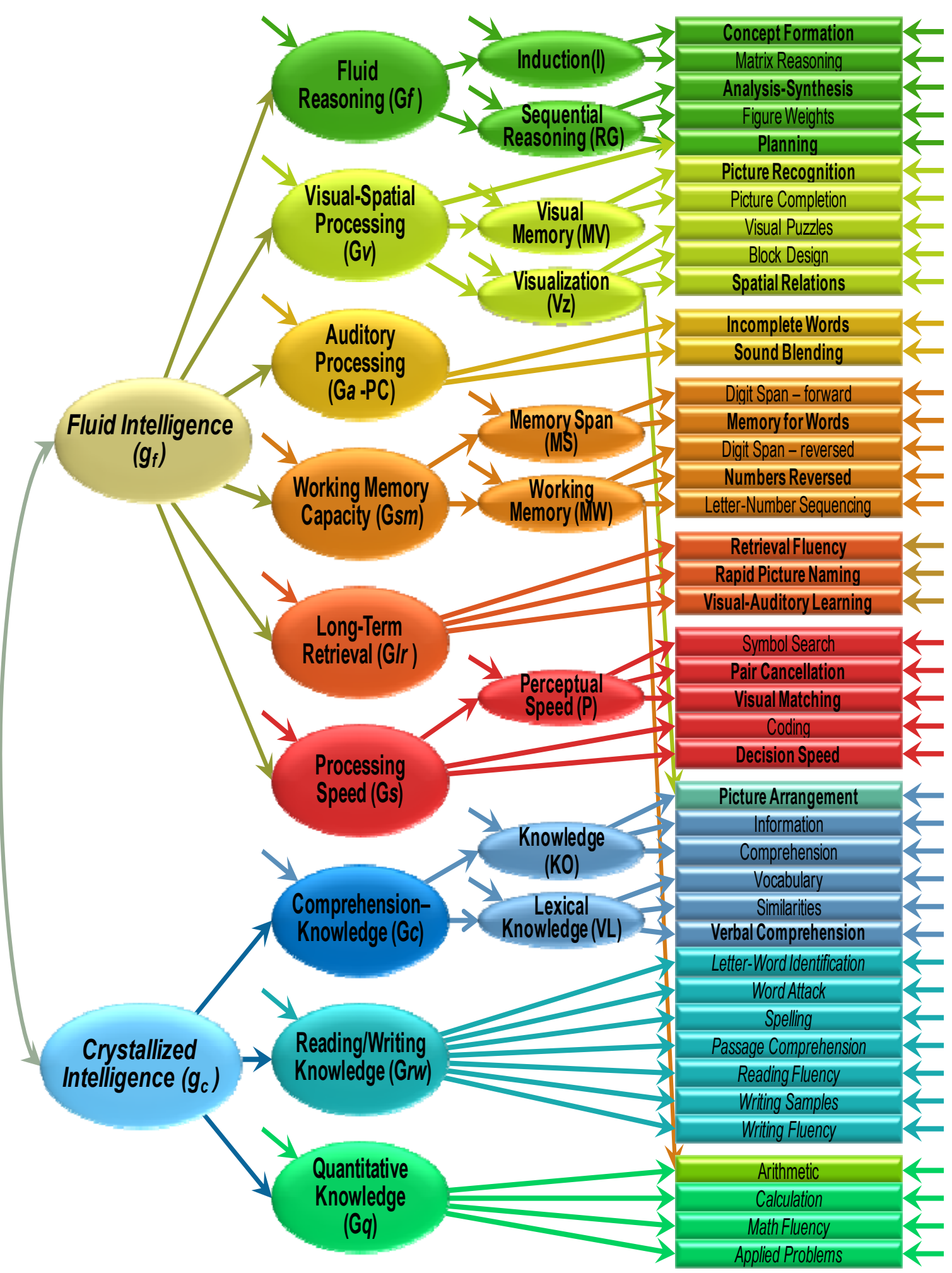

Figure 4. WAIS-IV, WJ III COG, and WJ III ACH in $g_{f} g_{c}$ measurement model. WJ III COG tests are in bold; WJ III ACH tests are in italics. 


\section{Hypothesis 2 - Cognitive-Achievement Relations}

Several recent studies have implicated $\mathrm{CHC}$ broad and narrow factors in the development of academic competency in school-aged populations (see McGrew \& Wendling, 2010, for a meta-analysis of 134 studies regarding reading and mathematics performance, and Floyd, Evans, \& McGrew, 2008, for a study regarding writing), but relatively fewer studies have investigated these relations in a college sample with suspected disabilities (e.g., Canivez \& Watkins, 2010; Gropper \& Tannock, 2009; Maller \& McDermott, 1997; Rohde \& Thompson, 2006; Spinks, Arndt, Caspers, Yucuis, McKirgan, Pfalzgraf, \& Waterman, 2007; Osmon, Braun, \& Plambeck, 2005; Osmon, Smerz, Braun, \& Plambeck, 2006; Proctor, 2012; Trainin \& Swanson, 2005). Using structural equation modeling, I analyzed the cognitive-achievement relations of this sample group within a $\mathrm{CHC}$ theory framework to determine whether the relations found in other studies fit this sample. Figures 5 through 8 illustrate structural models that were used to test the possible cognitive-achievement relations of this sample. The analyses from McGrew and Wendling (2010) and Floyd and colleagues 2008 reflect performances of the analyzed tests' standardization samples of school-aged children. The studies regarding college populations varied somewhat in their results. For example, Osmon and colleagues (2006) attributed mathematical processes to be directly influenced by the CHC broad factors of auditory processing $(\mathrm{G} a)$, visual-spatial processing $(\mathrm{G} v)$, and fluid reasoning $(\mathrm{G} f)$; and by the indirect factors of long-term memory and retrieval $(\mathrm{G} l r)$, working-memory capacity (Gsm), and comprehension-knowledge (Gc) through the global intelligence factor $g$. Proctor (2012) found support for the direct influences of processing speed $(\mathrm{G} s)$ and working-memory capacity (Gsm) on mathematical calculation, and the 
direct influences of comprehension-knowledge, fluid reasoning, and working memory capacity on mathematical reasoning. Therefore, the cognitive-achievement relations in this sample were expected to differ slightly from those found in the other studies, not only because of age differences between this sample and school-aged samples, but also because findings in other studies have been somewhat variable.

McGrew and Wendling (2010) and Floyd and colleagues (2008) highlighted the cognitive factors responsible for the achievement skills measured by the WJ III ACH, but they did not elaborate on whether individual cognitive abilities are directly responsible for complex skills or whether simple skills mediate in any way the relation between cognitive abilities and complex skills. Further, some studies find indirect influences of some cognitive abilities only through the global factor $g$. This analysis examined, for this sample, the direct contribution of cognitive abilities in the acquisition of complex skills and the extent to which their contribution is indirect through the acquisition of basic skills or through the global factor.

Although Figures 5 through 8 illustrate only structural models, they are intended to represent measurement models derived from the cognitive tests that load onto the various broad and narrow factors. The structural models were illustrated for simplicity's sake. In all model representations, all cognitive tests were analyzed to determine any possible loadings onto the various broad and narrow factors. Figures 5 and 6 represent models, one each for reading/writing and mathematics, in which there is no mediation of basic skills on cognitive abilities' influences on advanced skills. Figures 7 and 8 each show an example of how $\mathrm{g}$, broad, and narrow factors were tested to find the relations among basic and advanced skills in reading/writing and mathematics, respectively. 


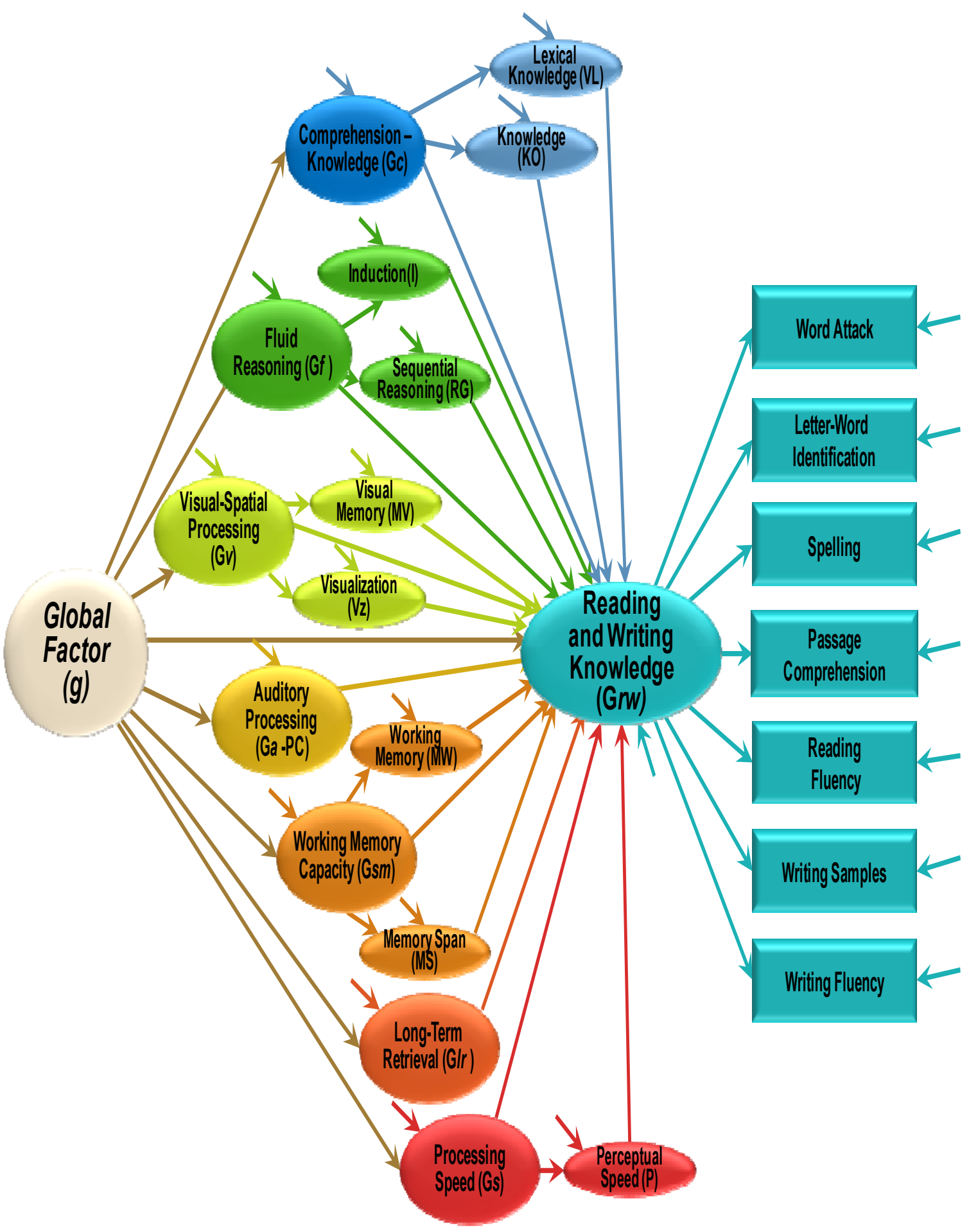

Figure 5. Hypothesis $2 \mathrm{a}(1)$ : For reading and writing achievement, there is no mediation of basic skills in the relation between cognitive abilities and advanced skills. This model tests all broad and narrow factors measured by the cognitive tests. 


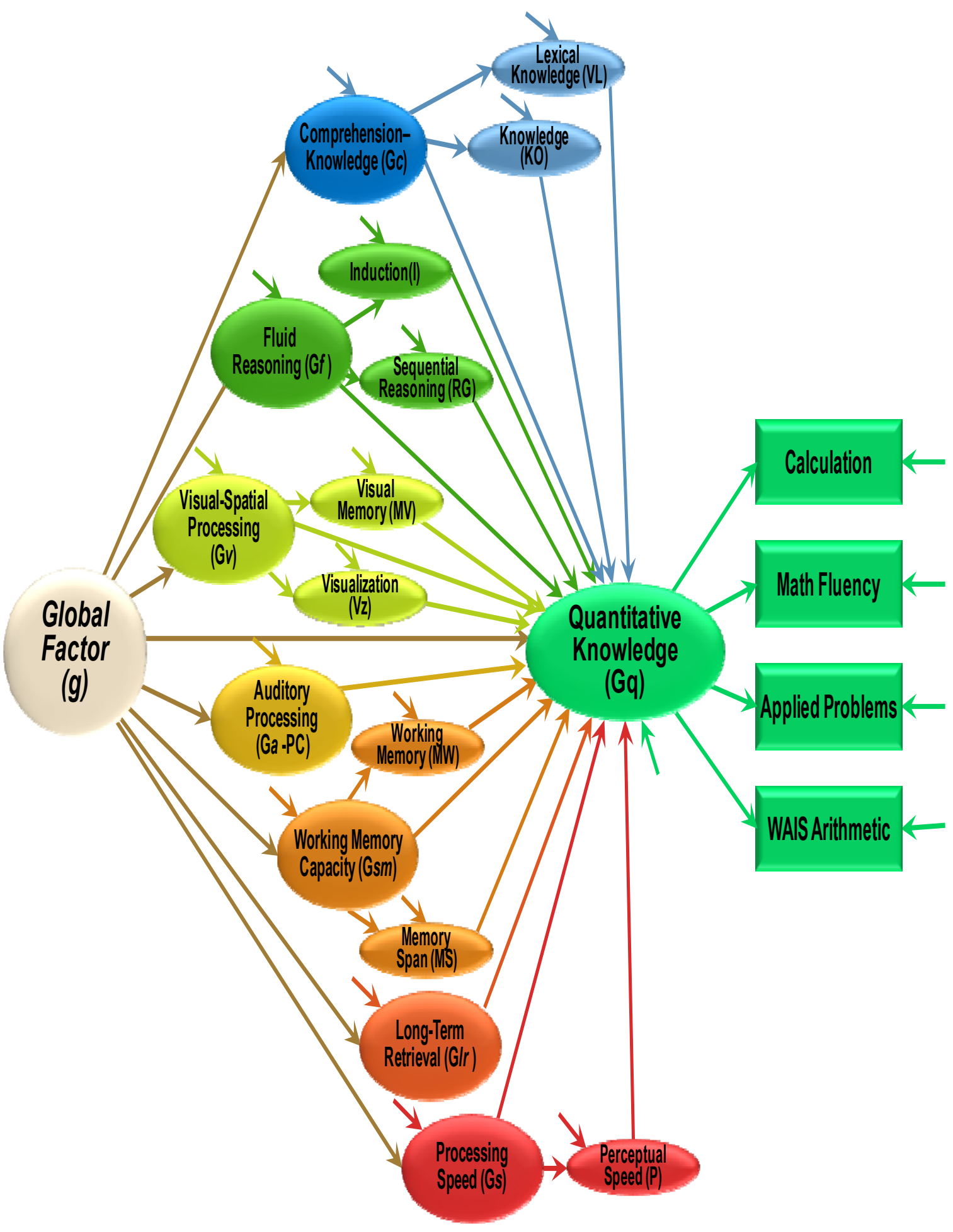

Figure 6. Hypothesis 2a(2): For mathematics achievement, there is no mediation of basic skills in the relations between cognitive abilities and advanced skills. This model includes all broad and narrow factors measured by the cognitive tests. 


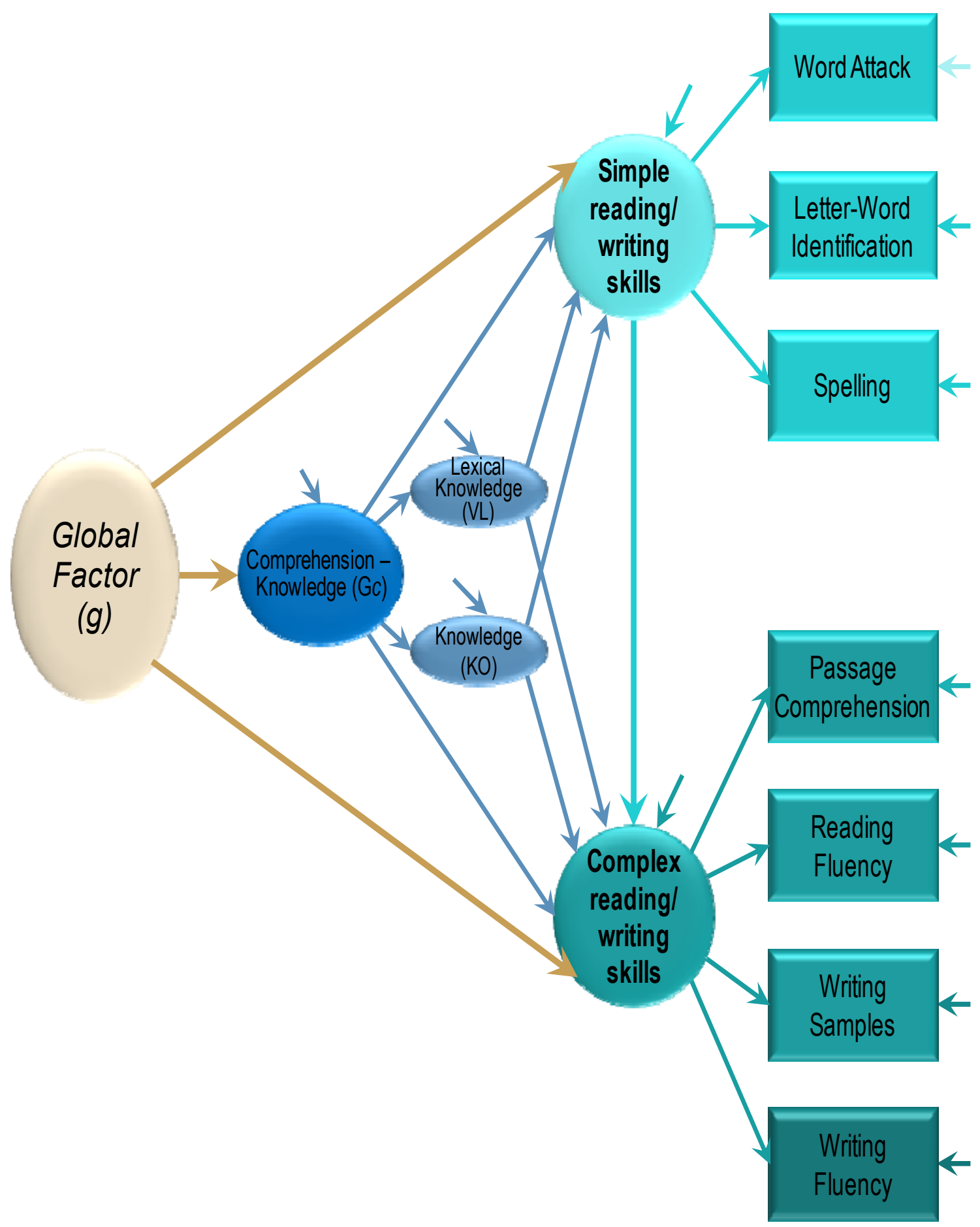

Figure 7. Hypothesis 2b(1): For reading/writing achievement, the extent to which basic skills mediate the relations between cognitive abilities and advanced skills was tested. This model includes one example of a CHC broad factor and narrow factors measured by the cognitive tests. All other factors were similarly tested. 


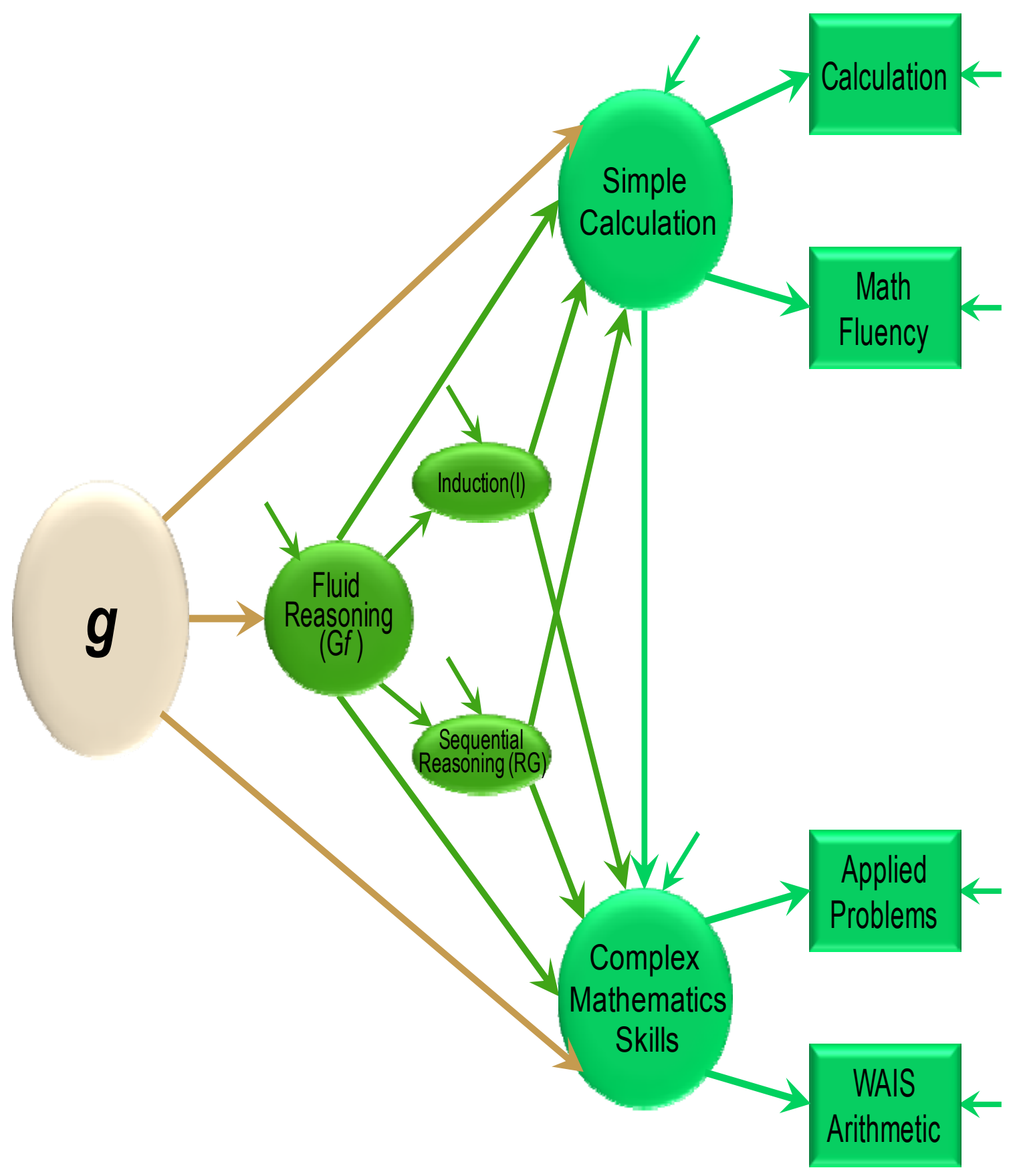

Figure 8. Hypothesis 2b(2): For mathematics achievement, the extent to which basic skills mediate the relations between cognitive abilities and advanced skills was tested. This model includes one example of a $\mathrm{CHC}$ broad factor and narrow factors measured by the cognitive tests. All other factors were similarly tested. 


\section{Hypothesis 3 - Consistent Incremental Validity of Additional Test Scores Regardless of Subtest Score Variability}

When two subtest scores within a composite are discrepant, some assessment experts recommend administering additional tests to validate the composite score (Fiorello, Hale, \& Wycoff, 2012; Flanagan, Alfonso, \& Ortiz, 2012). During cognitive assessment, clinicians often choose not to rely on the composite score if two or more subtests within the composite differ markedly (Schneider, in press), and they often administer additional subtests to enhance the validity of the composite score.

Alternatively, clinicians finding consistent scores within a composite often assume their composite score is valid and do not test further. Schneider (2011a) demonstrated, however, how additional assessment is just as likely to enhance the validity of consistent scores as discrepant scores. There are several reasons composite subtest scores might differ, including unique narrow ability factors that are not shared by the common factor, differences in subtest loadings on the common factor, and possible moderators that might affect factor loadings.

I hypothesized that the composite score will be similarly predictive of the composite ability assessed regardless of the discrepancy between the two scores. The current dataset allowed this hypothesis to be tested because scores from both cognitive batteries measuring similar CHC narrow and broad abilities could be compared. Consistent and discrepant scores within a composite could be compared to subtest scores in another battery to determine the incremental validity of the additional scores in both cases. The extent to which additional subtests change the original composite score may suggest in which circumstances their administration is worth the clinician's and client's 
time and effort. Using cross product regression analysis, four specific comparisons were used to determine the extent of incremental validity when additional subtests assessing the same broad factors are added. Table 1 lists the comparable subtests from each battery.

Table 1

Comparable Cognitive Abilities Subtests and Their Broad-Narrow Factors

\begin{tabular}{llc}
\hline \multicolumn{1}{c}{ WAIS-III/IV Subtest } & WJ III COG Subtest & Broad-Narrow Abilities \\
\hline Matrix Reasoning & Concept Formation & Gf-I \\
WAIS-IV Figure Weights & Analysis-Synthesis & Gf-RG/RQ \\
Digit Span - Digits Forward & Memory for Words & Gsm-MS \\
Digit Span - Digits Backward & Numbers Reversed & Gsm-MW \\
Letter-Number Sequencing & & Gsm-MW \\
\hline
\end{tabular}

Note. Figure Weights administered with the WAIS-IV only. G $f=$ Fluid Reasoning; Gsm = Short-Term Memory; I = Induction; RG/RQ = Sequential Reasoning/ Quantitative Reasoning; MS = Memory Span; MW = Working Memory Capacity.

\section{Hypothesis 4 - The Benefit of Service Utilization on GPA}

Taking advantage of offered services can help college students with ADHD, learning disabilities, and psychological disorders more successfully negotiate their college curricula (Allsop, Minskoff, and Bolt, 2005). Effects are often influenced, however, by certain characteristics of the student receiving the services. Allsop and colleagues (2005) found support for three elements that influence student outcomes that were examined in this dataset: students' cognitive abilities and initial academic achievement, psychological health, and dosage of services, defined as whether the student accessed an accommodation letter to receive classroom accommodations and by the number of coaching or counseling sessions the student attended at the disability resources center. I hypothesized that students' cognitive abilities, the extent of students' initial psychological difficulties, and the extent to which students accessed services at the 
disability resources center would predict the positive effects of a student's use of the disabilities center's resources. I proposed using structural equation modeling to determine the degree to which the following variables influenced academic outcomes over time, measured by students' grade point average (GPA) slopes for three semesters post-assessment:

- Cognitive abilities, measured by the WAIS-III/IV full-scale IQ (FSIQ) and WJ III General Intellectual Ability (GIA)

- Achievement, measured by the WJ III ACH and initial GPA

- Initial psychological health, as assessed by the Symptom Checklist-90Revised (SCL-90-R; Derogatis, 1994), Beck Depression Inventory, Second Edition (BDI-II; Beck, Steer, \& Brown, 1996), Conners Adult ADHD Rating Scale (CAARS; Conners, Erhardt, \& Sparrow,1998) Self-Concept Scale, and whether a Structured Clinical Interview for the DSM-IV Patient Edition, with Psychotic Screen (SCID-I/P W/ PSY SCREEN; First, Spitzer, Gibbon, \& Williams, 2002) was administered

- Dosage, assessed by the extent to which the student accessed services offered by the disability resource center and/or picked up a letter from the disability center allowing the student to receive academic accommodations (accommodation letter)

Figure 9 illustrates the hypothesized model for the effects of service utilization on college academic achievement. The model illustrates the direct effects of cognitive ability on standardized achievement and initial GPA, the direct effects of initial mental health on initial GPA and coaching attendance, and the indirect influence that cognitive 
ability and initial achievement had on service utilization's ability to affect GPA slope.

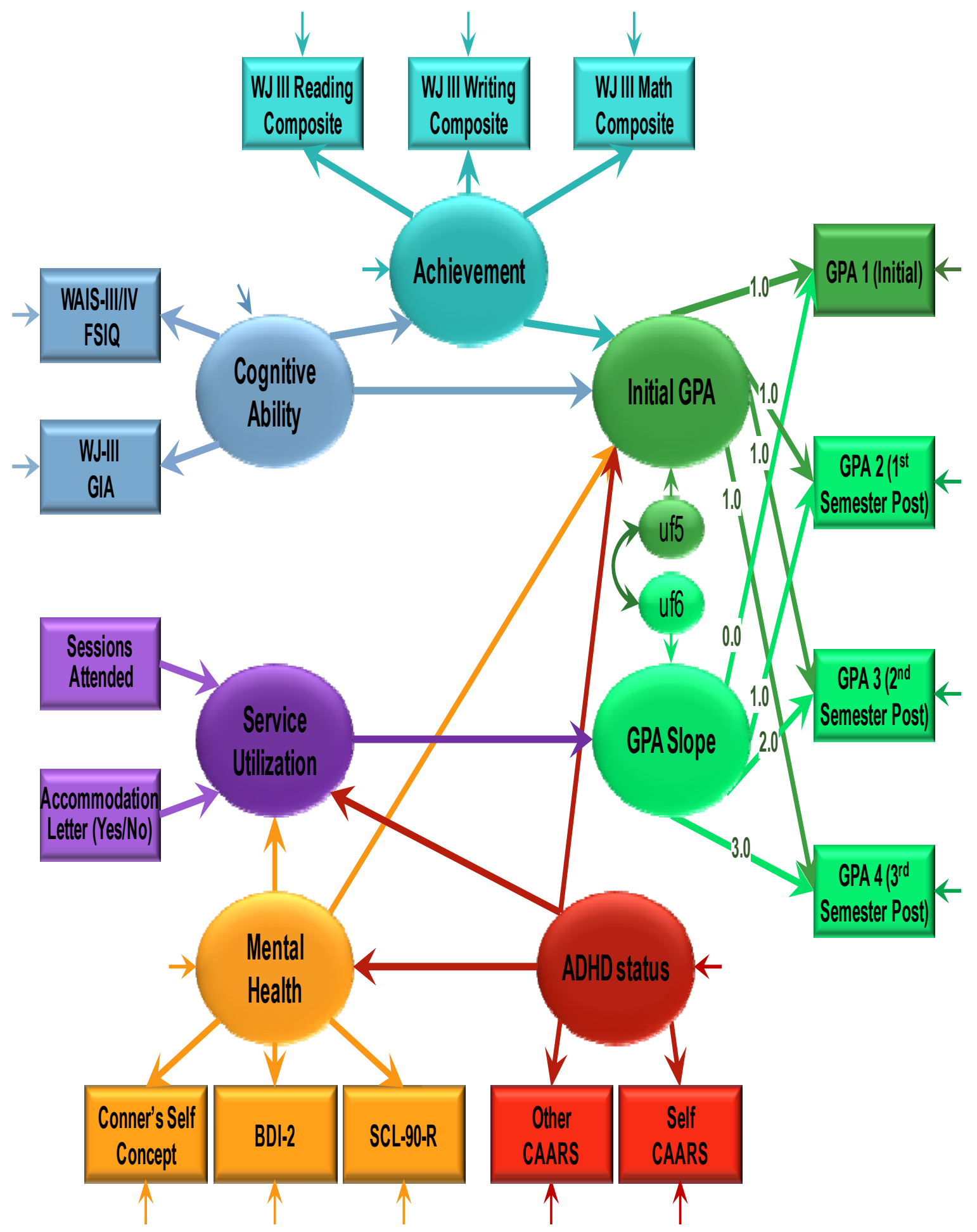

Figure 9. Hypothesized model of service utilization's effects on GPA slope. 


\section{CHAPTER III}

\section{METHOD}

\section{Participants}

This study used de-identified archival data obtained from a database of the disabilities resource center of a large, Midwestern, public university. Table 2 provides descriptive data for the sample. Demographic data and test scores were obtained from 2000 to 2012 from undergraduate and graduate college students self-referred or referred by university faculty or other staff for a comprehensive neuropsychological evaluation of attention, learning, or psychological difficulties. In addition, depending on psychological symptom severity, clients were given either a structured clinical interview or rating scales that assessed general emotional functioning and possible symptoms of depression. Participants provided consent at the time of their evaluations to have their results used anonymously in research. Because students were tested as part of a comprehensive evaluation and not primarily for research purposes, however, test order was not counterbalanced. 
Table 2

Groups Profiled by Cognitive, Achievement, and Diagnostic Tests

\begin{tabular}{lcc}
\hline \multicolumn{1}{c}{ Group } & $n$ & Percent \\
\hline Demographic Groups & & \\
Male & 706 & 54.6 \\
Female & 586 & 45.4 \\
Caucasian & 779 & 60.3 \\
African American & 191 & 14.8 \\
Asian/Indian & 183 & 14.2 \\
Latino & 133 & 10.3 \\
& & Percent of Students \\
Diagnoses (Includes comorbid diagnoses) & & Diagnosed \\
ADHD-Combined Type (ADHD-C) & 455 & 35.2 \\
Depression & 325 & 27.5 \\
ADHD-Inattentive Type (ADHD-I) & 314 & 24.3 \\
Anxiety & 249 & 19.3 \\
Verbal Learning Disability (VLD) & 203 & 15.7 \\
No Diagnosis & 141 & 10.9 \\
Nonverbal Learning Disability (NVLD) & 137 & 10.6 \\
Foreign Language Learning Difficulty (FLLD) & 86 & 6.7 \\
\hline
\end{tabular}

Each evaluation was conducted over eight to twelve hours by clinical or school psychology graduate student clinicians or postdoctoral fellows who had received formal course training in standardized test administration, scoring, and interpretation. The graduate clinicians also received ongoing assessment training and supervision by the director of the center, a licensed clinical psychologist with expertise in assessment and rehabilitation.

Students with suspected disabilities were administered one of two standard batteries depending on the severity of their psychological symptoms as assessed by a preliminary academic screening interview conducted by a graduate clinician. Upon completion of the 90-120 minute interview, the interviewer and center director discussed results and made a joint decision whether to administer a more structured clinical 
interview. For more severely symptomatic students for whom additional testing was indicated, SCID-I/P W/ PSY SCREEN modules assessing substance use, mood, anxiety, psychotic, and eating disorders, were administered in addition to the cognitive and other assessment measures. Clinicians also supplemented the SCID-I/P W/ PSY SCREEN modules with relevant sections from the Personality Disorder Interview for DSM-IV (PDI-IV; Widiger, Mangine, Corbitt, Ellis, \& Thomas, 1995) to assess additional symptoms of schizophrenia or emotion regulation difficulties, such as affective instability, difficulty controlling anger, and impulsivity. If, in the judgment of the clinical director and interviewer, psychological symptoms presented during the initial academic screening did not warrant a full clinical interview, clinicians administered the SCL-90-R and the BDI-II rating scales to evaluate students' general level of psychological distress and possible symptoms of depression. Several students, particularly during the time when the WAIS-III was used, were administered both the SCID and the rating scales.

Student clients were diagnosed with one or more disorders based on diagnostic criteria from the DSM-IV-TR (American Psychiatric Association, 2000). Diagnoses were made by the director of the center by using clinical judgment to interpret results of the student client's academic screening interview and comprehensive evaluation.

Selection criteria for this study included student clients of the center who received a complete battery of tests regardless of emotional symptom status. Exclusion criteria included students who did not receive both cognitive batteries and the achievement measure. Participants were identified from the database as having completed the WAIS- 
III or WAIS-IV, selected subtests of the WJ III COG and WJ III ACH, and the CAARS. The final sample $(N=1,292)$ included self- or other-referred students who met the study criteria. The WAIS-III dataset included the test scores from 1,040 students $(80.5 \%)$, and the WAIS-IV dataset consisted of the test scores from 252 students (19.5\%). An exploration of the demographic data revealed that the mean age of participants was 23.27 years $(S D=5.28)$. Men comprised $54.6 \%$ of the sample $(N=706)$ and women comprised $45.4 \%(N=586)$. Table 3 presents descriptive statistics on the diagnostic groups for this sample.

As an outcome of their evaluations, students were diagnosed with ADHD, Verbal (similar to DSM-IV Reading Disorder or Disorder of Written Expression), Nonverbal or other Learning Disability, mood or other psychological Disorder, or no disability. Regarding diagnoses of ADHD, 769 students (59.5\%) met DSM-IV diagnostic criteria for a primary diagnosis of ADHD, including 455 students (35.2\%) with ADHD-C, and 314 students (24.3\%) with ADHD-I. Diagnoses are reported in Table 3. The number of diagnoses exceeds the sample number because a sizable number of students were diagnosed with multiple disorders. 
Table 3

Diagnoses Represented in the Sample $(N=1,982)$

\begin{tabular}{lcr}
\hline \multicolumn{1}{c}{ Diagnosis } & Frequency & Percent \\
\hline ADHD, Combined (ADHD-C) & 455 & 22.96 \\
Major Depressive Disorder (MDD) & 331 & 16.70 \\
ADHD, Primarily Inattentive Type (ADHD-I) & 314 & 15.84 \\
Generalized Anxiety Disorder (GAD) & 249 & 12.56 \\
Verbal Learning Disability (VLD) & 203 & 10.24 \\
No Diagnosis & 141 & 7.11 \\
Nonverbal Learning Disability (NVLD) & 137 & 6.91 \\
Cognitive Disability NOS & 31 & 1.27 \\
Substance Use Disorder & 17 & 0.86 \\
Asperger's Syndrome & 16 & 0.82 \\
Bipolar Disorder & 13 & 0.66 \\
Reading Disorder & 12 & 0.61 \\
Eating Disorder & 9 & 0.45 \\
Personality Disorder & 9 & 0.45 \\
Learning Disability NOS & 8 & 0.40 \\
Math Disorder & 8 & 0.40 \\
Sleep Disorder & 6 & 0.30 \\
Adjustment Disorder & 4 & 0.20 \\
Obsessive-Compulsive Disorder (OCD) & 4 & 0.20 \\
Schizophrenia & 3 & 0.15 \\
ADHD, Hyperactive-Impulsive (ADHD-HI) & 3 & 0.15 \\
Psychosis & 3 & 0.15 \\
Disorder of Written Expression & 3 & 0.15 \\
Paranoia & 1 & 0.05 \\
Tourette's Syndrome & 1 & 0.05 \\
Speech Disorder & 1 & 0.05 \\
\hline Note. ADHD=Atention deficit hyperactivity & & \\
\hline
\end{tabular}

Note. $\mathrm{ADHD}=$ Attention deficit hyperactivity disorder; NOS $=$ Not otherwise specified

\section{Measures}

Measures were selected based on their reliability and validity with the college student population. Evaluations included a comprehensive battery of neuropsychological measures including the WAIS-III or, when it became available, the WAIS-IV, selected subtests of the WJ III COG and WJ III ACH, the long-form versions of the CAARS Self and Observer Reports (CAARS-S:L and CAARS-O:L, respectively); the SCL-90-R), the 
BDI-II, and The Trail Making Tests A and B (Reitan, 1986), tests of overall brain function.

\section{Woodcock-Johnson III (WJ III)}

WJ III subtests administered to this sample included the co-normed, individually administered WJ III COG and WJ III ACH. The WJ III COG is commonly used to assess general intellectual ability, seven broad $\mathrm{CHC}$ abilities, and many narrow aspects of the broad abilities. The WJ III COG is an updated version of the Woodcock-Johnson Psychoeducational Battery-Revised Tests of Cognitive Ability (WJ-R COG), the development of which was guided by one of the precursors of $\mathrm{CHC}$ theory, $\mathrm{G} f$ - $\mathrm{G} c$ theory (Mather \& Woodcock, 2001c).

The first cognitive assessment measure based specifically on CHC theory, the WJ III COG comprises a Standard Battery which includes seven standard and three supplemental subtests, and an Extended Battery which includes 10 subtests (Mather \& Woodcock, 2001b). Specific subsets of subtests within the Standard and Extended Batteries measure the Stratum III General Intellectual Ability (GIA; psychometric “ $g$ ”), seven Stratum II broad abilities including fluid reasoning $(\mathrm{G} f)$, comprehensionknowledge $(\mathrm{Gc})$, visual-spatial processing $(\mathrm{G} v)$, auditory processing $(\mathrm{G} a)$, long-term storage and retrieval $(\mathrm{G} l r)$, short-term memory $(\mathrm{G} s m)$, processing speed $(\mathrm{G} s)$, and an array of Stratum I narrow abilities, described in Table 4.

Each Stratum II cluster is assessed by at least two subtests that measure qualitatively different Stratum I abilities. Although each subtest of the WJ III COG was originally designed to measure only one Stratum II broad ability, subsequent factor 
analytic studies have demonstrated that several subtests tap into additional broad factors (McGrew, 2011; Schneider \& McGrew, 2012). Further, although each subtest measures at least one Stratum I narrow ability, several subtests tap into more than one (Schrank \& Wendling, 2009; Schrank \& Wendling, 2012). Of the 20 subtests available in the Standard and Extended batteries of the WJ III COG, 16 were administered to this sample and are described in Table 4. Omitted subtests include Auditory Working Memory, Visual-Auditory Learning-Delayed, General Information, and Auditory Attention.

The WJ III ACH consists of a Standard and Extended Battery and is available in two forms (A and B). Only selected subtests from Form A were administered to this sample. Although the WJ III ACH subtests cover five broad curricular clusters including reading, mathematics, written language, oral language, and academic knowledge, only subtests from the reading, mathematics, and written language areas were administered to this sample. Each cluster contains subtests that measure basic skills, fluency, and application. Of the 22 available subtests, this sample was administered 9 of 12 subtests from the Standard Battery: Letter-Word Identification, Reading Fluency, Passage Comprehension, Calculation, Math Fluency, Applied Problems, Spelling, Writing Fluency, and Writing Samples; and 1 of 10 subtests from the Extended Battery: Word Attack. Descriptions of the WJ III ACH subtests are found in Table 5.

The WJ III was originally normed on a comparatively large sample of more than 8,800 examinees from age 2 to $90+$ years. This sample provided a balanced cross-section of individuals that approximated the 2000 U.S. Census as closely as possible on variables such as gender, race/ethnicity, and geographic area (Schrank, McGrew, \& Woodcock, 
2001). The WJ III Normative Update (WJ III NU) was created in 2007 from 8,782 of the original WJ III norming subjects by recalculating normative data and updating norm construction of the WJ III based on population changes reflected in the 2005 census. This update was representative with regard to variables including census region, community size, gender, race, whether the examinee was Hispanic or foreign born, type of college, adult occupational status, and adult educational level. The WJ III NU also provides information regarding the validity of inferred cognitive processes based on updated research (McGrew, Schrank, \& Woodcock, 2007). The WJ III measures administered to this sample were scored using the WJ III NU norms.

The WJ III NU technical manual (McGrew, Schrank, \& Woodcock, 2007) and independent reviews of the WJ III COG and WJ III ACH (Cizek, 2003; Sandoval, 2003) indicate that subtest and composite scores are reliable and valid measures of cognitive abilities and achievement for all the ages tested in the sample. Odd and even split-half procedures determined the WJ III NU median reliability scores for all but the speeded tests and tests with multiple-point scoring systems (Schrank \& Wendling, 2012). The reliabilities for speeded tests and tests with multiple-point scoring systems were determined using Rasch analysis procedures. Only two cognitive tests (Picture Recognition at .76 and Planning at .74) and one achievement test (Writing Samples at .75 ) exhibit median reliabilities less than .80 . All but three median cluster reliabilities are .90 or higher. The lowest median cluster reliabilities include Long-Term Retrieval (.88), Visual-Spatial Thinking (.81), and Short-Term Memory (.88; Schrank \& Wendling, 2012). Median reliabilities for all cluster scores and for the WJ III cognitive and 
achievement subtests administered to this study's sample are listed in Table 6.

A major objective of WJ III development was to align the battery with CHC theory and decrease subtest variances not attributable to the narrow ability being measured by a particular subtest (Schrank \& Wendling, 2012). The content and construct validities of the WJ III have been supported by numerous exploratory and confirmatory factor analyses with tests from the WJ III and other well-established cognitive and achievement batteries (Keith \& Reynolds, 2010; McGrew, Schrank, \& Woodcock, 2007; Schrank \& Wendling, 2012). McGrew and Woodcock (2001c) reported solid evidence in the technical manual for the efficacy of the WJ III to identify students with learning disabilities and provide helpful diagnostic information in the assessment of students with ADHD, giftedness, and intellectual disabilities. Studies indicating high correlations between WJ III subtests that measure similar abilities and lower correlations between subtests measuring different abilities suggest strong internal structural validity (McGrew \& Woodcock, 2001b). For example, in the WJ III NU 20 to 39 year-old age group (the age group with the most relevance for this study's sample), the correlation between the two Gc subtests of Verbal Comprehension and Information is .84, but the correlation between the Gc subtest Verbal Comprehension and the Gs subtest Decision Speed is only .32 (McGrew, Schrank, \& Woodcock, 2007). Studies that include comparisons of the WJ III subtests to similar and dissimilar constructs in other intelligence and achievement measures also demonstrate strong convergent, divergent, and concurrent validity (Mather \& Woodcock, 2001c) 


\section{Wechsler Adult Intelligence Scales (WAIS)}

Graduate clinicians administered the complete core and supplementary subtests of the WAIS-III to this sample until 2010, when they began to administer all but the Cancellation subtest of the WAIS-IV. The WAIS-III and WAIS-IV are individually administered, norm-referenced tests commonly used to measure general intellectual ability. As the precursor of the WAIS-IV, the WAIS-III is composed of 14 subtests of which 11 are used to compute the FSIQ. The WAIS-IV comprises 15 subtests of which 10 core subtests are used to compute the FSIQ. The FSIQ and indexes each have a mean of 100 and a standard deviation of 15 . Subtest scaled scores have a mean of 10 and a standard deviation of 3 (Lichtenberger \& Kaufman, 2009). In addition to the FSIQ, the WAIS-III subtests were constructed to measure four distinct cognitive domains (Zhu \& Weiss, 2005). The Verbal Comprehension Index comprises the subtests Vocabulary, Similarities, Information, and Comprehension. As a measure of comprehensionknowledge $(\mathrm{Gc})$, the Verbal Comprehension Index assesses one's ability to reason using previously acquired information through the use of lexical knowledge and verbal reasoning. The Perceptual Organization Index employs the subtests of Block Design, Matrix Reasoning, Picture Completion, and the supplemental test of Picture Arrangement to assess the non-verbal skills of fluid reasoning, spatial processing, and visual-motor integration.

The Working Memory Index encompasses the tests of Arithmetic, Digit Span, and Letter-Number Sequencing to assess one's ability to manipulate temporarily stored incoming information. The Processing Speed Index is composed of the subtests Digit- 
Symbol Coding and Symbol Search and is a measure of the rapidity with which one can perform easy tasks accurately. Object Assembly is an optional subtest that was not used in the calculation of index scores but could contribute to the FSIQ. It assesses one's ability to fit together, within a time limit, pieces of a puzzle to form a meaningful whole (Zhu \& Weiss, 2005). Object Assembly was not administered to this sample.

Most of the WAIS-III subtests remain in the WAIS-IV; some new subtests have been added, however, and some old subtests were removed in the construction of the WAIS-IV. New subtests for the WAIS-IV include three new subtests in the newly named Perceptual Reasoning Index (PRI, replacing the POI): Visual Puzzles, a variation of the WAIS-III Object Assembly subtest; and Figure Weights and Cancellation, added as supplemental subtests. Cancellation was not administered to most of this sample.

Additional modifications to the WAIS-IV include the deletion of Picture Arrangement and Object Assembly to reduce the emphasis on time bonus points and decrease the potential confound of test motor demands (Lichtenberger \& Kaufman, 2009). Some minor optional WAIS-III scores were also omitted from the WAIS-IV (i.e., Digit-Symbol-Copy and Digit-Symbol-Incident Learning) while others were added (i.e., Process scores in Block Design, Digit Span, and Letter-Number Sequencing) to promote qualitative assessment of test-taking performance. Digit-Symbol Coding became simply Coding and the tests are similar. Regardless of the changes made to the WAIS-IV, correlations to the WAIS-III remain between .84 to .91 on the subtests and .94 for the FSIQ (Drozdick, Wahlstrom, Zhu, \& Weiss, 2012). The WAIS-III and WAIS-IV tests and the CHC factors on which they load are described in Table 4. 
The WAIS-III and WAIS-IV were standardized on samples of 2,450 and 2,200 adults, respectively, divided into 13 age bands and closely approximating the U.S. Census on such demographic variables as age, gender, race and ethnicity, educational level, and geographic region (The Psychological Corporation, 1997; Drozdick et al., 2012). Both batteries exhibit excellent psychometric properties (Zhu \& Weiss, 2005; Drozdick et al., 2012). Both WAIS measures exhibit strong reliability, with internal-consistency reliability coefficients in the .90 range for index scores and .98 for the FSIQ. Core subtest-level internal-consistency reliability coefficients are also strong, in the $.80 \mathrm{~s}$ or .90 s for the normative group and from .80 to .90 for clinical samples. The measures demonstrate test-retest reliability coefficients ranging from .87 to .96 and subtest stability coefficients between .74 and .90 (The Psychological Corporation, 1997; Drozdick et al., 2012). Similarly, the measures' construct validities are supported by factor-analytic studies which support the test creators' four cognitive factors of Verbal Comprehension Index (VCI), Perceptual Organization/Perceptual Reasoning Indices (POI/PRI), Working Memory Index (WMI), and Processing Speed Index (PSI) (Drozdick et al., 2012; Zhu \& Weiss, 2005). Additionally, the content validity of the WAIS-IV has been enhanced by revisions that better reflect the $\mathrm{CHC}$ theoretical framework (Drozdick et al., 2012; Schneider \& McGrew, 2012), although factor analytic studies on the WAIS-III also indicate a structure that is aligned with CHC theory (Golay \& Lecerf, 2011). Other independent confirmatory factor-analytic research demonstrates the WAIS-IV's alignment with CHC theory (Benson, Hulac, \& Kranzler, 2010). 
Table 4

Relations of Wechsler and WJ III COG Tests and CHC Abilities

\begin{tabular}{|c|c|c|}
\hline $\begin{array}{l}\text { Subtest }(\mathrm{CHC} \\
\text { Broad Ability) }\end{array}$ & $\begin{array}{l}\text { CHC Narrow } \\
\text { Ability }\end{array}$ & $\begin{array}{c}\text { Inferred Cognitive Processes Measured/Test } \\
\text { Requirements }\end{array}$ \\
\hline $\begin{array}{l}\text { WJ III Verbal } \\
\text { Comprehension } \\
(\mathrm{Gc})\end{array}$ & $\begin{array}{l}\text { Lexical knowledge/ } \\
\text { Language } \\
\text { Development (VL- } \\
\text { LD)** }\end{array}$ & $\begin{array}{l}\text { Includes four subtests requiring verbal } \\
\text { responses: Picture Vocabulary assesses } \\
\text { lexical knowledge by requiring evaluee to } \\
\text { recognize and identify pictures; Synonyms } \\
\text { and Antonyms assess vocabulary knowledge } \\
\text { by requiring evaluee to activate, access, and } \\
\text { match semantic knowledge; and Verbal } \\
\text { Analogies assesses analogical reasoning skills } \\
\text { using lexical knowledge. }\end{array}$ \\
\hline $\begin{array}{l}\text { WAIS-III/IV } \\
\text { Vocabulary }(\mathrm{Gc})\end{array}$ & $\begin{array}{l}\text { Lexical knowledge/ } \\
\text { Language } \\
\text { Development (VL- } \\
\text { LD)** }\end{array}$ & $\begin{array}{l}\text { Assesses vocabulary understanding in terms } \\
\text { of correct word meanings by requiring } \\
\text { evaluee to define orally presented words. }\end{array}$ \\
\hline $\begin{array}{l}\text { WAIS-III/IV } \\
\text { Information }(\mathrm{Gc})\end{array}$ & $\begin{array}{l}\text { General verbal } \\
\text { information (KO); } \\
\text { Lexical knowledge/ } \\
\text { Language } \\
\text { Development (VL- } \\
\text { LD)** }\end{array}$ & $\begin{array}{l}\text { Assesses range of general knowledge and } \\
\text { language understanding by requiring evaluee } \\
\text { to give brief answers to questions about a } \\
\text { variety of general knowledge topics. }\end{array}$ \\
\hline $\begin{array}{l}\text { WAIS-III/IV } \\
\text { Comprehension } \\
(\mathrm{Gc})\end{array}$ & $\begin{array}{l}\text { General verbal } \\
\text { information (KO); } \\
\text { Lexical knowledge/ } \\
\text { Language } \\
\text { Development (VL- } \\
\text { LD)** }\end{array}$ & $\begin{array}{l}\text { Assesses range of general knowledge, } \\
\text { practical reasoning and judgment, and } \\
\text { language understanding by requiring evaluee } \\
\text { to answer questions pertaining to everyday } \\
\text { problems and social situations. }\end{array}$ \\
\hline $\begin{array}{l}\text { WAIS-III/IV } \\
\text { Similarities }(\mathrm{Gc})\end{array}$ & $\begin{array}{l}\text { Lexical knowledge/ } \\
\text { Language } \\
\text { Development (VL- } \\
\text { LD)** }\end{array}$ & $\begin{array}{l}\text { Assesses language understanding and verbal } \\
\text { reasoning by requiring evaluee to explain the } \\
\text { similarity between a pair of orally presented } \\
\text { words. }\end{array}$ \\
\hline
\end{tabular}

Assesses inductive reasoning ability by

WJ III Concept

Formation $(\mathrm{G} f)$

Induction (I)

WAIS-III/IV

Matrix Induction (I)

Reasoning (Gf) requiring evaluee to identify, categorize, and switch rules when given feedback of response correctness.

Assesses inductive reasoning ability by requiring evaluee to identify the missing part of a series of visually presented matrices. 
Table 4

Relations of Wechsler and WJ III COG Tests and CHC Abilities, continued

\begin{tabular}{|c|c|c|}
\hline $\begin{array}{l}\text { Subtest (CHC } \\
\text { Broad Ability) }\end{array}$ & $\begin{array}{l}\text { CHC Narrow } \\
\text { Ability }\end{array}$ & $\begin{array}{c}\text { Inferred Cognitive Processes Measured/Test } \\
\text { Requirements }\end{array}$ \\
\hline $\begin{array}{l}\text { WJ III Analysis- } \\
\text { Synthesis (Gf) }\end{array}$ & $\begin{array}{l}\text { General sequential } \\
\text { reasoning (RG); } \\
\text { Quantitative } \\
\text { reasoning (RQ) }\end{array}$ & $\begin{array}{l}\text { Assesses deductive reasoning ability by } \\
\text { requiring evaluee to determine the missing } \\
\text { components of symbolic puzzles and evaluee } \\
\text { is given feedback regarding response } \\
\text { correctness. }\end{array}$ \\
\hline $\begin{array}{l}\text { WAIS-IV Figure } \\
\text { Weights (Gf) }\end{array}$ & $\begin{array}{l}\text { General sequential } \\
\text { reasoning }(\mathrm{RG}) \text {; } \\
\text { Quantitative } \\
\text { reasoning (RQ) }\end{array}$ & $\begin{array}{l}\text { Assesses quantitative and analogical } \\
\text { reasoning, mental flexibility, and set shifting } \\
\text { ability by requiring evaluee to determine } \\
\text { numerical relationships between shapes and } \\
\text { select the correct response option with a } \\
\text { specified time limit. }\end{array}$ \\
\hline $\begin{array}{l}\text { WJ III Planning } \\
(\mathrm{G} f, \mathrm{G} v)\end{array}$ & $\begin{array}{l}\text { General sequential } \\
\text { reasoning (Gf- } \\
\mathrm{RG}) ; \text { Spatial } \\
\text { scanning }(\mathrm{G} v-\mathrm{SS})\end{array}$ & $\begin{array}{l}\text { Assesses means-end analysis by requiring } \\
\text { evaluee to trace a pattern without removing } \\
\text { the pencil from the paper or retracing any } \\
\text { lines. }\end{array}$ \\
\hline $\begin{array}{l}\text { WAIS-III/IV } \\
\text { Arithmetic (Gf; } \\
\text { Gsm) }\end{array}$ & $\begin{array}{l}\text { Quantitative } \\
\text { reasoning (Gf- } \\
\text { RQ); Working } \\
\text { memory capacity } \\
\text { (Gsm-MW) }\end{array}$ & $\begin{array}{l}\text { Assesses numerical reasoning ability and } \\
\text { auditory working memory by requiring } \\
\text { evaluee to solve a series of orally presented } \\
\text { arithmetic problems within a specified time } \\
\text { limit. }\end{array}$ \\
\hline $\begin{array}{l}\text { WJ III Spatial } \\
\text { Relations }(\mathrm{G} v)\end{array}$ & Visualization (Vz) & $\begin{array}{l}\text { Assesses visual feature/image detection, } \\
\text { matching, and manipulation in space by } \\
\text { requiring evaluee to identify correct subsets } \\
\text { of pieces that form target shapes. }\end{array}$ \\
\hline $\begin{array}{l}\text { WAIS-III/IV } \\
\text { Block Design } \\
(\mathrm{G} v)\end{array}$ & Visualization (Vz) & $\begin{array}{l}\text { Assesses visual feature detection, matching, } \\
\text { and manipulation in space by requiring } \\
\text { evaluee to reproduce a series of designs on } \\
\text { blocks that have been visually presented on } \\
\text { paper. }\end{array}$ \\
\hline $\begin{array}{l}\text { WAIS-IV Visual } \\
\text { Puzzles }(\mathrm{G} v)\end{array}$ & Visualization (Vz) & $\begin{array}{l}\text { Assesses visual feature detection, matching, } \\
\text { and manipulation by requiring evaluee to } \\
\text { reproduce a geometric image by choosing the } \\
\text { correct subset of visually presented pieces. }\end{array}$ \\
\hline
\end{tabular}


Table 4

Relations of Wechsler and WJ III COG Tests and CHC Abilities, continued

\begin{tabular}{|c|c|c|}
\hline $\begin{array}{l}\text { Subtest (CHC } \\
\text { Broad Ability) }\end{array}$ & $\begin{array}{l}\text { CHC Narrow } \\
\text { Ability }\end{array}$ & $\begin{array}{c}\text { Inferred Cognitive Processes Measured/Test } \\
\text { Requirements }\end{array}$ \\
\hline $\begin{array}{l}\text { WJ III Picture } \\
\text { Recognition }(\mathrm{G} v)\end{array}$ & $\begin{array}{l}\text { Visual memory } \\
(\mathrm{MV})\end{array}$ & $\begin{array}{l}\text { Assesses the formation of memories and } \\
\text { ability to match visual stimuli to stored } \\
\text { representations by requiring evaluee to } \\
\text { identify a subset of previously presented } \\
\text { pictures within a field of similar distracting } \\
\text { pictures. }\end{array}$ \\
\hline $\begin{array}{l}\text { WAIS-III/IV } \\
\text { Picture } \\
\text { Completion }(\mathrm{G} v)\end{array}$ & $\begin{array}{l}\text { Visual memory } \\
(\mathrm{MV})\end{array}$ & $\begin{array}{l}\text { Assesses ability to recognize or recall } \\
\text { previously formed and stored mental images } \\
\text { by requiring evaluee to identify a part that is } \\
\text { missing from a common picture. }\end{array}$ \\
\hline $\begin{array}{l}\text { WJ III Sound } \\
\text { Blending }(\mathrm{G} a \text {; } \\
\mathrm{Gc})\end{array}$ & $\begin{array}{l}\text { Phonetic coding } \\
\text { (PC); Lexical } \\
\text { knowledge (VL) }\end{array}$ & $\begin{array}{l}\text { Assesses ability to match sound sequences to } \\
\text { stored, accessed, and recalled lexical } \\
\text { knowledge by requiring evaluee to synthesize } \\
\text { dictated discrete language sounds } \\
\text { (phonemes). }\end{array}$ \\
\hline $\begin{array}{l}\text { WJ III } \\
\text { Incomplete } \\
\text { Words }(\mathrm{G} a)\end{array}$ & $\begin{array}{l}\text { Phonetic coding } \\
\text { (PC) }\end{array}$ & $\begin{array}{l}\text { Assesses auditory analysis and closure of } \\
\text { acoustic sequences by requiring evaluee to } \\
\text { identify dictated words that have missing } \\
\text { phonemes. }\end{array}$ \\
\hline $\begin{array}{l}\text { WJ III Visual- } \\
\text { Auditory } \\
\text { Learning }(\mathrm{G} l r)\end{array}$ & $\begin{array}{l}\text { Associative } \\
\text { memory (MA); } \\
\text { Meaningful } \\
\text { memory (MM); } \\
\text { Glr-Learning } \\
\text { Efficiency** }\end{array}$ & $\begin{array}{l}\text { Assesses learning and recall of visual- } \\
\text { auditory associations by requiring evaluee to } \\
\text { learn and recall pictographic representations } \\
\text { of orally presented words. }\end{array}$ \\
\hline $\begin{array}{l}\text { WJ III Retrieval } \\
\text { Fluency }(\mathrm{G} l r)\end{array}$ & $\begin{array}{l}\text { Ideational fluency } \\
\text { (FI); Naming } \\
\text { facility (NA); Glr- } \\
\text { Retrieval } \\
\text { Fluency*** }\end{array}$ & $\begin{array}{l}\text { Assesses the recognition, retrieval fluency, } \\
\text { and oral production of examples of a semantic } \\
\text { category by requiring evaluee to name } \\
\text { verbally as many examples as possible from a } \\
\text { given well-known category. }\end{array}$ \\
\hline $\begin{array}{l}\text { WJ III Rapid } \\
\text { Picture Naming } \\
(\mathrm{G} s ; \mathrm{G} l r)\end{array}$ & $\begin{array}{l}\text { Glr- Retrieval } \\
\text { Fluency***; } \\
\text { Naming facility } \\
\text { (NA) }\end{array}$ & $\begin{array}{l}\text { Assesses speed, retrieval fluency, and oral } \\
\text { production of recognized objects by requiring } \\
\text { evaluee to retrieve from memory and orally } \\
\text { name as rapidly as possible drawings of well- } \\
\text { known objects. }\end{array}$ \\
\hline
\end{tabular}


Table 4

Relations of Wechsler and WJ III COG Tests and CHC Abilities, continued

\begin{tabular}{|c|c|c|}
\hline $\begin{array}{l}\text { Subtest (CHC } \\
\text { Broad Ability) }\end{array}$ & $\begin{array}{l}\text { CHC Narrow } \\
\text { Ability }\end{array}$ & $\begin{array}{c}\text { Inferred Cognitive Processes Measured/Test } \\
\text { Requirements }\end{array}$ \\
\hline $\begin{array}{l}\text { WJ III Decision } \\
\text { Speed } \\
(\mathrm{G} s ; \mathrm{G} l r)\end{array}$ & $\begin{array}{l}\text { Glr-Retrieval } \\
\text { Fluency*** }\end{array}$ & $\begin{array}{l}\text { Assesses speed of recognizing objects and } \\
\text { making symbolic/semantic comparisons by } \\
\text { requiring evaluee to locate and circle as } \\
\text { quickly as possible the two most conceptually } \\
\text { similar pictures in a row of pictures. }\end{array}$ \\
\hline $\begin{array}{l}\text { WJ III Visual } \\
\text { Matching (Gs) }\end{array}$ & $\begin{array}{l}\text { Perceptual speed } \\
(\mathrm{P}) \text {; Number } \\
\text { facility }(\mathrm{N})\end{array}$ & $\begin{array}{l}\text { Assesses speed at which visual symbols can } \\
\text { be perceived and matched by requiring } \\
\text { evaluee to locate and circle as quickly as } \\
\text { possible two identical numbers in a given row } \\
\text { of numbers. }\end{array}$ \\
\hline
\end{tabular}

WJ III Pair

Cancellation

$(\mathrm{G} s)$

Perceptual speed

Assesses vigilance and attentional control by requiring evaluee to identify and circle as quickly as possible occurrences of a repeated pattern within rows of similar but unidentical distracters.

Assesses speed and accuracy of search,

WAIS-III/IV

Symbol Search

(Gs)

Perceptual speed

(P) comparison, and identification of visual elements separated in a visual field by requiring evaluee to scan as quickly as possible a row of symbols and identify matching symbols in the group.

Assesses speed and accuracy of search,

WAIS-III

Cancellation

(Gs)

Perceptual speed comparison, and identification of visual elements presented side-by-side by requiring evaluee to scan and mark target pictures within a time limit.

Assesses rapid performance of an easy task

WAIS-III/IV Rate of test taking Coding (Gs) (R9)

WJ III Numbers Working memory Reversed (Gsm; $\mathrm{Gs}$ ) capacity (GsmMW); Number facility $(\mathrm{G} s-\mathrm{N})$ by requiring evaluee to draw symbols that are paired with a series of numbers according to a key.

Assesses ability to temporarily encode, maintain, and manipulate numerical information by requiring evaluee to hold an increasing span of numbers in immediate awareness while reversing the sequence. 
Table 4

Relations of Wechsler and WJ III COG Tests and CHC Abilities, continued

\begin{tabular}{|c|c|c|}
\hline $\begin{array}{l}\text { Subtest (CHC } \\
\text { Broad Ability) }\end{array}$ & $\begin{array}{l}\text { CHC Narrow } \\
\text { Ability }\end{array}$ & $\begin{array}{c}\text { Inferred Cognitive Processes Measured/Test } \\
\text { Requirements }\end{array}$ \\
\hline $\begin{array}{l}\text { WAIS-III/IV } \\
\text { Digit Span- } \\
\text { Reversed (Gsm; } \\
\text { Gs) }\end{array}$ & $\begin{array}{l}\text { Working memory } \\
\text { capacity (MW); } \\
\text { Number facility }\end{array}$ & $\begin{array}{l}\text { Assesses ability to temporarily encode, } \\
\text { maintain, and manipulate numerical } \\
\text { information by requiring evaluee to hold an } \\
\text { increasing span of numbers in immediate } \\
\text { awareness while reversing the sequence. }\end{array}$ \\
\hline $\begin{array}{l}\text { WAIS-III/IV } \\
\text { Letter-Number } \\
\text { Sequencing } \\
\text { (Gsm) }\end{array}$ & $\begin{array}{l}\text { Working memory } \\
\text { capacity (MW) }\end{array}$ & $\begin{array}{l}\text { Assesses ability to temporarily encode, } \\
\text { maintain, and manipulate information by } \\
\text { requiring evaluee to orally recall numbers in } \\
\text { ascending order and letters in alphabetical } \\
\text { order after hearing sequences of random } \\
\text { letters and numbers. }\end{array}$ \\
\hline $\begin{array}{l}\text { WJ III Memory } \\
\text { for Words (Gsm) }\end{array}$ & Memory span (MS) & $\begin{array}{l}\text { Assesses ability to encode, maintain, and } \\
\text { immediately recall temporally ordered } \\
\text { elements by requiring evaluee to repeat in the } \\
\text { correct sequence an orally presented list of } \\
\text { unrelated words. }\end{array}$ \\
\hline $\begin{array}{l}\text { WAIS-III/IV } \\
\text { Digit Span - } \\
\text { Forward (Gsm) }\end{array}$ & Memory span (MS) & $\begin{array}{l}\text { Assesses ability to encode, maintain, and } \\
\text { immediately recall temporally ordered } \\
\text { elements by requiring evaluee to repeat in the } \\
\text { correct sequence an orally presented list of } \\
\text { numbers. }\end{array}$ \\
\hline
\end{tabular}

Note. Narrow abilities measured by only one test are subsumed under the corresponding CHC broad ability.

* Also denotes an intermediate ability of Language Development between CHC Broad and Narrow abilities of $\mathrm{Gc}$ and $\mathrm{VC} / \mathrm{LD}$, respectively.

** Denotes an intermediate ability between CHC Broad and Narrow abilities, tapping efficiency of encoding, storing, and recalling more information than can be retained in Gsm (Schneider \& McGrew, 2012).

***Denotes an intermediate ability between CHC Broad and Narrow abilities, tapping lexical access speed (McGrew, 2011; Schneider \& McGrew, 2012). 
Table 5

Relations of WJ III ACH Tests, Related Curricular Areas, and CHC Abilities

Test/Curricular CHC Broad/ Area Narrow Ability

\begin{tabular}{|c|c|c|}
\hline $\begin{array}{l}\text { Letter-Word } \\
\text { Identification/ } \\
\text { Reading }\end{array}$ & $\begin{array}{l}\text { Grw/Reading } \\
\text { decoding (RD) }\end{array}$ & $\begin{array}{l}\text { Assesses word identification skill by } \\
\text { requiring evaluee to identify and read } \\
\text { isolated letters and words. }\end{array}$ \\
\hline $\begin{array}{l}\text { Reading } \\
\text { Fluency/Reading }\end{array}$ & $\begin{array}{l}\text { Gs; Grw/Reading } \\
\text { speed (RS); } \\
\text { Semantic processing } \\
\text { speed (Gs-P) }\end{array}$ & $\begin{array}{l}\text { Assesses skill at quickly reading and } \\
\text { understanding simple sentences by } \\
\text { requiring evaluee to circle "yes" or "no" } \\
\text { within a time limit to a question about } \\
\text { each sentence read. }\end{array}$ \\
\hline $\begin{array}{l}\text { Calculation/ } \\
\text { Mathematics }\end{array}$ & $\begin{array}{l}\text { Gq/Mathematical } \\
\text { achievement (A3) }\end{array}$ & $\begin{array}{l}\text { Assesses calculation knowledge and skill } \\
\text { by requiring evaluee to solve increasingly } \\
\text { complex mathematics equations. }\end{array}$ \\
\hline $\begin{array}{l}\text { Math Fluency/ } \\
\text { Mathematics }\end{array}$ & $\begin{array}{l}\text { Gq/Mathematical } \\
\text { achievement (A3); } \\
\text { Number facility (Gs- } \\
\text { N) }\end{array}$ & $\begin{array}{l}\text { Assesses ability to solve single-digit } \\
\text { addition, subtraction, and multiplication } \\
\text { facts quickly. }\end{array}$ \\
\hline Spelling/Spelling & $\begin{array}{l}\text { Grw/Spelling ability } \\
\text { (SG) }\end{array}$ & $\begin{array}{l}\text { Assesses ability to write orally presented } \\
\text { words correctly. }\end{array}$ \\
\hline $\begin{array}{l}\text { Writing Fluency/ } \\
\text { Writing }\end{array}$ & $\begin{array}{l}\text { Grw/Writing ability } \\
\text { (WA); Writing speed } \\
\text { (Gps-WS) }\end{array}$ & $\begin{array}{l}\text { Assesses ability to create and write short, } \\
\text { simple sentences quickly by requiring } \\
\text { evaluee to use three visually presented } \\
\text { words and relate each sentence to a } \\
\text { stimulus picture within a time limit. }\end{array}$ \\
\hline $\begin{array}{l}\text { Passage } \\
\text { Comprehension/ } \\
\text { Reading }\end{array}$ & $\begin{array}{l}\text { Grw / Reading } \\
\text { comprehension }(\mathrm{RC})\end{array}$ & $\begin{array}{l}\text { Assesses reading comprehension by } \\
\text { requiring evaluee to read a short passage } \\
\text { and supply a missing word that makes } \\
\text { contextual sense. }\end{array}$ \\
\hline $\begin{array}{l}\text { Applied Problems/ } \\
\text { Mathematics }\end{array}$ & $\begin{array}{l}\text { Gq/Quantitative } \\
\text { reasoning; } \\
\text { Mathematical } \\
\text { achievement (A3); } \\
\text { Mathematical } \\
\text { knowledge (KM) }\end{array}$ & $\begin{array}{l}\text { Assesses mathematical knowledge and } \\
\text { reasoning skills by requiring evaluee to } \\
\text { complete verbally presented practical } \\
\text { story problems. }\end{array}$ \\
\hline
\end{tabular}


Table 5

Relations of WJ III ACH Tests, Related Curricular Areas, and CHC Abilities, continued Test/Curricular CHC Broad/ Area Narrow Ability

Test Description/Requirement

\begin{tabular}{lll}
\hline $\begin{array}{l}\text { Writing } \\
\text { Samples/Writing }\end{array}$ & $\begin{array}{l}\text { Grw/Writing } \\
\text { ability (WA) }\end{array}$ & $\begin{array}{l}\text { Assesses expressive writing for expressive } \\
\text { quality, but not for basic writing skills such } \\
\text { as spelling or punctuation, by requiring } \\
\text { evaluee to write sentences in response to } \\
\text { increasingly complex prompts. }\end{array}$ \\
$\begin{array}{l}\text { Gord } \\
\text { Attack/Reading }\end{array}$ & $\begin{array}{l}\text { Gecoding (RD) } \\
\text { Phonetic coding } \\
(\mathrm{G} a \text {-PC) }\end{array}$ & $\begin{array}{l}\text { Assesses decoding skills by requiring } \\
\text { evaluee to pronounce visually presented } \\
\text { phonically regular nonwords. }\end{array}$ \\
$\begin{array}{l}\text { Letter-Word } \\
\text { Identification/ } \\
\text { Reading }\end{array}$ & $\begin{array}{l}\text { Grw/Reading } \\
\text { decoding (RD) }\end{array}$ & $\begin{array}{l}\text { Assesses word identification skill by } \\
\text { requiring evaluee to identify and read } \\
\text { isolated letters and words. }\end{array}$ \\
\hline
\end{tabular}

Table 6

Subtest/Cluster Median Reliability Statistics of the WJ III NU Cognitive and Achievement Subtests

\begin{tabular}{lcc}
\multicolumn{1}{c}{ Test or Cluster } & Median $r_{11}$ & Median SEM (SS) \\
\hline Cognitive Standard Battery & & \\
\hline Verbal Comprehension & .92 & 4.24 \\
Visual-Auditory Learning & .86 & 5.61 \\
Spatial Relations & .81 & 6.54 \\
Sound Blending & .89 & 4.97 \\
Concept Formation & .94 & 3.67 \\
Visual Matching & .88 & 5.24 \\
Numbers Reversed & .87 & 5.41 \\
Incomplete Words & .81 & 6.54 \\
Cognitive Extended Battery & & \\
Retrieval Fluency & .85 & 5.81 \\
Picture Recognition & .76 & 7.35 \\
Analysis-Synthesis & .90 & 4.74 \\
Decision Speed & .88 & 5.16 \\
Memory for Words & .80 & 6.71 \\
Rapid Picture Naming & .97 & 2.51 \\
Planning & .74 & 7.65 \\
Pair Cancellation & .96 & 2.92
\end{tabular}


Table 6

Subtest/Cluster Median Reliability Statistics of the WJ III NU Cognitive and Achievement Subtests, continued

\begin{tabular}{|c|c|c|}
\hline Test or Cluster & Median $r_{11}$ & Median SEM (SS) \\
\hline \multicolumn{3}{|l|}{ Achievement Standard Battery } \\
\hline Letter-Word Identification & .94 & 3.67 \\
\hline Reading Fluency & .95 & 3.27 \\
\hline Calculation & .86 & 5.61 \\
\hline Math Fluency & .98 & 2.36 \\
\hline Spelling & .90 & 4.74 \\
\hline Writing Fluency & .83 & 6.24 \\
\hline Passage Comprehension & .88 & 5.20 \\
\hline Applied Problems & .93 & 3.97 \\
\hline Writing Samples & .75 & 7.52 \\
\hline \multicolumn{3}{|l|}{ Achievement Extended Battery } \\
\hline Word Attack & .87 & 5.41 \\
\hline \multicolumn{3}{|l|}{ Cognitive Cluster Standard Battery } \\
\hline General Intellectual Ability - Standard & .97 & 2.60 \\
\hline Verbal Ability - Standard & .92 & 4.24 \\
\hline Thinking Ability - Standard & .95 & 3.35 \\
\hline Cognitive Efficiency - Standard & .91 & 4.50 \\
\hline Phonemic Awareness (PC) & .90 & 4.74 \\
\hline Working Memory (WM) & .91 & 4.50 \\
\hline \multicolumn{3}{|l|}{ Cognitive Cluster Extended Battery } \\
\hline General Intellectual Battery & .98 & 2.12 \\
\hline Verbal Ability - Extended & .95 & 3.35 \\
\hline Thinking Ability - Extended & .96 & 3.00 \\
\hline Cognitive Efficiency - Extended & .92 & 4.24 \\
\hline Comprehension-Knowledge ( $G c)$ & .95 & 3.35 \\
\hline Long-Term Retrieval (Glr) & .88 & 5.20 \\
\hline Visual-Spatial Thinking (Gv) & .81 & 6.54 \\
\hline Auditory Processing $(G a)$ & .91 & 4.50 \\
\hline Fluid Reasoning $(G f)$ & .95 & 3.35 \\
\hline Processing Speed (Gs) & .92 & 4.24 \\
\hline Short-Term Memory (Gsm) & .88 & 5.20 \\
\hline Broad Attention & .94 & 3.67 \\
\hline Cognitive Fluency & .96 & 3.00 \\
\hline Executive Processes & .96 & 3.00 \\
\hline Phonemic Awareness (PC) & .90 & 4.74 \\
\hline
\end{tabular}


Table 6

Subtest/Cluster Median Reliability Statistics of the WJ III NU Cognitive and Achievement Subtests, continued

\begin{tabular}{lcc}
\multicolumn{1}{c}{ Test or Cluster } & Median $r_{11}$ & Median SEM (SS) \\
\hline Standard Battery Achievement Cluster & & \\
\hline Total Achievement & .98 & 2.12 \\
Broad Reading & .96 & 3.00 \\
Broad Math & .95 & 3.35 \\
Broad Written Language & .92 & 4.24 \\
\hline
\end{tabular}

Note. Copyright (C) 2007 by the Riverside Publishing company. Woodcock-Johnson ${ }^{\circledR}$ III Normative Update (WJ III ${ }^{\circledR}$ NU, WJIII NU Technical Manual reproduced with permission of the publisher. All rights reserved.

\section{Conners' Adult ADHD Rating Scales (CAARS)}

The CAARS are reliable and valid measures of ADHD-related behaviors and symptoms in adults ages 18 to 50+ (Conners et al., 1999). The measures are available in two types of forms: the self-report CAARS:S to be completed by the client, and the observer report CAARS:O to be completed by someone who knows the client well, such as a friend or family member. The two forms assess the same behaviors and difficulties and contain identical scales, subscales, and indexes. The CAARS is available in long (66 items, CAARS:S:L or CAARS:O:L) and short (23 items, CAARS:S:S or CAARS:O:S) forms. In this sample, students were given the long-form versions of both the CAARS:S:L and the CAARS:O:L. They were instructed to complete the CAARS:S:L themselves during the evaluation and have a friend or family member complete the CAARS:O:L at another time and mail it to the center in a self-addressed stamped envelope that was given to the student along with the CAARS:O:L.

The long versions of the CAARS contain empirically derived scales that assess a wide range of behavior challenges including inattention and memory problems, 
hyperactivity, impulsivity, and poor self-concept. The Problems with Self Concept Scale has been found to be related to feelings of hopelessness, low self esteem, anxiety, and self-isolation and might indicate symptoms of comorbid depression, but more work is needed to clarify this scale with respect to its relationship with major depressive disorder (Conners, Erhardt, Epstein, Parker, Sitarenios, \& Sparrow, 1999).

The CAARS indicated good reliability for all age groups as measured by internal consistency, mean inter-item correlations, test-retest, and standard error of measurement/prediction. Tables $7,8,9,10$, and 11 show summaries of reliability information for 18-29 year olds.

Table 7

Internal Reliability Coefficients for CAARS:S:L and CAARS:O:L for 18-29 Year Olds

\begin{tabular}{lcccc}
\hline & \multicolumn{2}{c}{ CAARS:S:L } & \multicolumn{2}{c}{ CAARS:O:L } \\
& Men & Women & Men & Women \\
& $(N=117)$ & $(N=144)$ & $(N=136)$ & $(N=131)$ \\
\hline Inattention/Memory Problems & .88 & .89 & .87 & .88 \\
Hyperactivity/Restlessness & .86 & .87 & .92 & .91 \\
Impulsivity/Emotional Lability & .88 & .87 & .90 & .91 \\
Problems with Self Concept & .82 & .81 & .84 & .80 \\
ADHD Index & .81 & .84 & .86 & .89 \\
DSM-Inattentive Symptoms & .64 & .65 & .82 & .80 \\
DSM-Hyperactive/Impulsive Symptoms & .78 & .86 & .90 & .89 \\
\hline
\end{tabular}

Note. Copyright (C) 1998, Multi-Health Systems Inc. All rights reserved. In the U.S.A., P.O. Box 950, North Tonawanda, NY 14120-0950, (800) 456-3003. In Canada, 3770 Victoria Park Ave., Toronto, ON M2H 3M6, (800) 268-6011. International, +1-416492-2627. Fax, +1-416-492-3343 or (888) 540-4484. 
Table 8

Mean Inter-Item Correlations for CAARS:S:L and CAARS:O:L for 18-29 Year Olds

\begin{tabular}{lcccc}
\hline \multirow{1}{c}{ Scale } & \multicolumn{2}{c}{ CAARS:S:L } & \multicolumn{2}{c}{ CAARS:O:L } \\
& Men & Women & Men & Women \\
& $(N=117)$ & $(N=144)$ & $(N=136)$ & $(N=131)$ \\
\hline Inattention/Memory Problems & .40 & .40 & .41 & .48 \\
Hyperactivity/Restlessness & .38 & .42 & .38 & .39 \\
Impulsivity/Emotional Lability & .35 & .38 & .49 & .46 \\
Problems with Self Concept & .57 & .54 & .59 & .55 \\
ADHD Index & .28 & .27 & .31 & .26 \\
DSM-Inattentive Symptoms & .33 & .41 & .42 & .48 \\
DSM-Hyperactive/Impulsive Symptoms & .20 & .27 & .35 & .34 \\
DSM-Total ADHD Symptoms & .19 & .29 & .35 & .32 \\
\hline
\end{tabular}

Note. Copyright (C) 1998, Multi-Health Systems Inc. All rights reserved. In the U.S.A., P.O. Box 950, North Tonawanda, NY 14120-0950, (800) 456-3003. In Canada, 3770 Victoria Park Ave., Toronto, ON M2H 3M6, (800) 268-6011. International, +1-416492-2627. Fax, +1-416-492-3343 or (888) 540-4484.

Table 9

Test-Retest Correlations for CAARS:S:L and CAARS:O:L-Ages and Sexes Combined Scale CAARS:S:L $(N=61) \quad$ CAARS:O:L $(N=50)$ (One-Month Interval) (Two-Week Interval)

\begin{tabular}{lll}
\hline Inattention/Memory Problems & $.88^{*}$ & $.95^{*}$ \\
Hyperactivity/Restlessness & $.90^{*}$ & $.90^{*}$ \\
Impulsivity/Emotional Lability & $.80^{*}$ & $.90^{*}$ \\
Problems with Self Concept & $.91^{*}$ & $.87^{*}$ \\
ADHD Index & $.90^{*}$ & $.89^{*}$ \\
DSM-Inattentive Symptoms & $\mathrm{N} / \mathrm{A}$ & $.95^{*}$ \\
DSM-Hyperactive/Impulsive Symptoms & $\mathrm{N} / \mathrm{A}$ & $.90^{*}$ \\
DSM-Total ADHD Symptoms & $\mathrm{N} / \mathrm{A}$ & $.95^{*}$
\end{tabular}

Note. Copyright (C) 1998, Multi-Health Systems Inc. All rights reserved. In the U.S.A., P.O. Box 950, North Tonawanda, NY 14120-0950, (800) 456-3003. In Canada, 3770 Victoria Park Ave., Toronto, ON M2H 3M6, (800) 268-6011. International, +1-416492-2627. Fax, +1-416-492-3343 or (888) 540-4484.

$* p<.05$. The self-report test-retest study used a preliminary version of the CAARS$\mathrm{S}: \mathrm{L}$ with fewer items than the final CAARS forms. Therefore, test-retest correlations are available only on the main clinical scales. 
Table 10

Standard Error of Measurement Scores for CAARS:S:L and CAARS:O:L for 18-29 Year Olds

\begin{tabular}{lcccc}
\hline & \multicolumn{2}{c}{ CAARS:S:L } & \multicolumn{2}{c}{ CAARS:O:L } \\
\multicolumn{1}{c}{ Scale } & Men & Women & Men & Women \\
& $(N=117)$ & $(N=144)$ & $(N=136)$ & $(N=131)$ \\
\hline Inattention/Memory Problems & 2.23 & 2.13 & 2.31 & 2.11 \\
Hyperactivity/Restlessness & 2.54 & 2.39 & 2.47 & 2.42 \\
Impulsivity/Emotional Lability & 2.50 & 2.25 & 2.22 & 2.20 \\
Problems with Self Concept & 1.48 & 1.49 & 1.37 & 1.42 \\
ADHD Index & 2.64 & 2.60 & 2.58 & 2.57 \\
DSM-Inattentive Symptoms & 1.67 & 1.80 & 2.01 & 1.95 \\
DSM-Hyperactive/Impulsive Symptoms & 2.40 & 2.21 & 2.16 & 2.15 \\
DSM-Total ADHD Symptoms & 3.02 & 2.94 & 3.05 & 3.08 \\
\hline
\end{tabular}

Note. Copyright (C) 1998, Multi-Health Systems Inc. All rights reserved. In the U.S.A., P.O. Box 950, North Tonawanda, NY 14120-0950, (800) 456-3003. In Canada, 3770 Victoria Park Ave., Toronto, ON M2H 3M6, (800) 268-6011. International, +1-416492-2627. Fax, +1-416-492-3343 or (888) 540-4484.

Table 11

Standard Error of Prediction (SEP) Scores for CAARS:S:L and CAARS:O:L for 18-29 Year Olds

\begin{tabular}{lcccc}
\hline & \multicolumn{2}{c}{ CAARS:S:L } & \multicolumn{2}{c}{ CAARS:O:L } \\
& Men & Women & Men & Women \\
& $(N=117)$ & $(N=144)$ & $(N=136)$ & $(N=131)$ \\
\hline Inattention/Memory Problems & 2.33 & 2.23 & 1.56 & 1.67 \\
Hyperactivity/Restlessness & 2.32 & 2.28 & 2.16 & 2.21 \\
Impulsivity/Emotional Lability & 2.99 & 2.79 & 2.49 & 2.32 \\
Problems with Self Concept & 1.28 & 1.24 & 1.56 & 1.48 \\
ADHD Index & 1.97 & 1.89 & 2.14 & 1.90 \\
DSM-Inattentive Symptoms & N/A & N/A & 1.20 & 1.32 \\
DSM-Hyperactive/Impulsive Symptoms & N/A & N/A & 1.61 & 1.52 \\
DSM-Total ADHD Symptoms & N/A & N/A & 2.16 & 2.08 \\
\hline
\end{tabular}

Note. Copyright (C) 1998, Multi-Health Systems Inc. All rights reserved. In the U.S.A., P.O. Box 950, North Tonawanda, NY 14120-0950, (800) 456-3003. In Canada, 3770 Victoria Park Ave., Toronto, ON M2H 3M6, (800) 268-6011. International, +1-416492-2627. Fax, +1-416-492-3343 or (888) 540-4484. SEP scores are based on CAARS test-retest reliability estimates and are therefore calculated from the test-retest values presented in Table 9. Scores are therefore unavailable for DSM-related measures. 
Validity information presented in the CAARS manual (Conners et al., 1999) indicates good factorial, discriminant, and convergent validity. A CFA of the various instruments showed good model fit with the four-factor structure of the CAARS-S:L subscales of Inattention/Memory Problems, Hyperactivity/ Restlessness, Impulsivity/Emotional Lability, and Problems with Self-Concept: The Non-Normed Fit Index (NNFI) was .983, and the Comparative Fit Index (CFI) was .984. Intercorrelations of the subscales support the multidimensional nature of the CAARS (Conners et al., 1999). Further, a discriminant validity evaluation indicated that the CAARS correctly classified persons with ADHD and non-ADHD controls $85 \%$ of the time. Regarding construct validity, the CAARS was compared to the Wender Utah Rating Scale (WURS; Ward et al., 1993), a scale assessing retrospective childhood symptomology. The scales would be expected to be associated with one another given the developmental nature of ADHD. As expected, Pearson product-moment correlations between the WURS total score and each of the four CAARS subscales were significant $(p<.01)$. Correlations between the self and observer reports ranged between .41 to .68 for the identical subscales, indicating an adequate relationship between the self and observer scales (Conners et al., 1999).

\section{Symptom Checklist-90-R (SCL-90-R)}

The SCL-90-R was administered to the study sample as a self-report psychological symptom inventory screening. The SCL-90-R was designed to assess a wide variety of psychopathological symptoms through nine subscales (Somatization, ObsessiveCompulsive, Interpersonal Sensitivity, Depression, Anxiety, Hostility, Phobic Anxiety, 
Paranoid Ideation, and Psychoticism) and three global indices: the Global Severity Index (GSI), the Positive Symptom Distress Index (PSDI), and the Positive Symptom Total (PST). The GSI is computed by summing all the scores on the nine symptom dimensions and additional items and then dividing the sum by the total number of responses (Derogatis, 1994). Although the author cited his own work to validate the nine symptom dimensions of the scale (Derogatis, 1994), subsequent independent factor analyses demonstrated strong loadings on only one primary factor, supporting the instrument as a valid measure of only one global dimension of psychological distress, reflected by the GSI (Brophy, Norvell, \& Kiluk, 1988; Schmitz, Hartkamp, Kruse, Franke, Reister, \& Tress, 2000; Schmitz, Kruse, Heckrath, Alberti, \& Tress, 1999; Vallejo, Jordán, Diaz, Comeche, \& Ortega, 2007). For this reason, only the GSI was reported in this study's sample as a general screening for psychological distress.

Originally normed on four samples of heterogeneous psychiatric and nonpsychiatric groups, the SCL-90-R exhibits adequate internal consistency and test-retest reliability. Internal consistency coefficient alphas for each of the nine dimensions of the instrument ranged from .77 to .90 with most alphas around .85 . The test-retest coefficients ranged from a low of .68 (Somatization was retested at 10 weeks for psychiatric outpatients) to .90 (Phobic Anxiety was retested at one week for heterogeneous psychiatric outpatients; Derogatis, 1994). Subsequent independent studies also attest to the high internal consistency of the SCL-90-R (Schmitz, Hartkamp, Kruse, Franke, Reister, \& Tress, 2000).

Validity studies support the SCL-90-R's ability to differentiate between subjects 
who have and have not been diagnosed with a psychological disorder (Schmitz et al., 2000; Schmitz, Kruse, Heckrath, Alberti, \& Tress, 1999; Vallejo, Jordán, Diaz, Comeche, \& Ortega, 2007). Brophy, Norvell, and Kiluk (1988) supported the convergent validity of the SCL-90-R when they found significant correlations between the BDI and relevant SCL-90-R symptom scales, and between scales of the MMPI and those of the SCL-90-R. High concurrent validity was found when comparing subscales of the SCL-90-R with the Inventory of Interpersonal Problems (IIP-C) and the General Health Questionnaire (GHQ-12; Vallejo, Jordán, Diaz, Comeche, \& Ortega, 2007), as well as comparing the SCL-90 as an overall psychological distress indicator with the Structured Clinical Interview for DSM Disorders (SCID; Schmitz, Kruse, Heckrath, Alberti, \& Tress; 1999). Numerous additional studies attesting to the convergent and divergent validity of the SCL-90-R are described in the technical manual (Derogatis, 1994).

\section{Beck Depression Inventory - Second Edition (BDI-II)}

The BDI-II is a 21-item self-report measure to assess depression severity in adolescents and adults aged 13 years and older (Beck, Steer, \& Brown, 1996). The BDIII was developed to correspond to criteria for depressive disorders listed in the DSM-IV (1994). Since its inception 35 years ago, the BDI has been widely used to assess symptom severity in clinical populations as well as to screen for possible depression in non-diagnosed individuals.

The current iteration of the BDI, the BDI-II, was created using item and factor analyses from a pilot-study revision of the BDI, the BDI-IA (Beck et al., 1996). Several items from the BDI-IA were revised to reflect analyses results, to accommodate the 
accumulating psychometric data of the previous 35 years, and to align more closely with the diagnostic criteria of the DSM-IV. Psychometric information of the BDI-II was gathered from the administration of the instrument to 500 psychiatric outpatients (317 women, 183 men; ages 13-86 years, 91\% White, 4\% African American, 4\% Asian American, and 1\% Hispanic) and to 120 college students as a comparative normal group (67 women, 53 men; mean age of 19.58 years $(S D=1.84)$, primarily White). The measure demonstrated good internal consistency, with coefficient alphas for corrected item-total correlations of .92 and .93 , respectively, for the clinical and control groups, and significance of $p<.05$ for all 21 items. Test-retest stability, based on a subsample of the clinical group being retested one week after the first administration, was demonstrated with a significant correlation of $.93(p<.001)$. Validity studies indicated good convergent and discriminant validity and are summarized in Table 11. In particular, good discriminant validity was shown between the BDI-II and measures of anxiety.

Table 12

Convergent/Discriminant Validity Correlations Between BDI-II and Other Measures

\begin{tabular}{lc}
\hline Scale & $r$ \\
\hline Beck Hopelessness Scale $(N=158)$ & .68 \\
Scale for Suicide Ideation $(N=158)$ & .37 \\
Beck Anxiety Inventory $(N=297)$ & .60 \\
Revised Hamilton Psychiatric Rating Scale for Depression $(N=87)$ & .71 \\
Revised Hamilton Anxiety Rating Scale $(N=87)$ & .47 \\
\hline
\end{tabular}

Note. Reproduced from the Manual of the Beck Depression Inventory-II (BDI-II). Copyright 1996 Aaron T. Beck. Reproduced with permission of the publisher NCS Pearson, Inc. All rights reserved. "Beck Depression Inventory" and (BDI) are trademarks, in the US and/or other countries, of Pearson Education, Inc. or its affiliate(s). All correlations were significant $<.001$, one-tailed test, after a Bonferroni adjustment of alpha/5 (Beck et al., 1996). 
Factor analyses of the BDI-II indicated a two-factor solution with healthy correlations between the two oblique factors of .66 $(p<.001)$ for the clinical sample and $.62(p<.001)$ for the control group (Beck et al., 1996). For the clinical group, items loading on the first factor included the Somatic/Affective symptoms of Loss of Pleasure, Crying, Agitation, Loss of Interest, Indecisiveness, Loss of Energy, Changes in Sleeping Pattern, Irritability, Changes in Appetite, Concentration Difficulty, Tiredness or Fatigue, and Loss of Interest in Sex. The other factor represented a Cognitive aspect of depression and comprised the items Sadness, Pessimism, Past Failure, Guilty Feelings, Punishment Feelings, Self-Dislike, Self-Criticalness, Suicidal Thoughts or Wishes, and Worthlessness. The control group of college students indicated a somewhat different factor structure: Because some items loaded on the two factors differently, the factors were considered for the control group to represent Somatic and Cognitive/Affective aspects of depression. Items switching factors for the control group included Loss of Pleasure, Crying, Agitation, Loss of Interest, Indecisiveness, and Irritability. Loss of Interest in Sex did not load significantly on either factor for the control group. A factor matching procedure indicated that, although the BDI-II comprises two highly correlated cognitive-affective and somatic aspects, particular affective symptoms may change loadings depending on the sample. Nevertheless, Beck and colleagues' (1996) discriminant validity study indicates that people diagnosed with depressive disorders obtain higher scores than individuals in the control group, and individuals with more severe symptomology obtain higher scores than individuals who exhibit less severe symptoms. 


\section{CHAPTER IV}

\section{RESULTS}

The purpose of this chapter is to present the results of analyses of the WAIS-III and WAIS-IV, the WJ III cognitive and achievement tests, the CAARS self- and otherrated scales, the BDI-II, and the SCL-90-R that were administered to a sample of college students with suspected disabilities. Through cross-tabulation chi-square analyses, independent sample t-tests, and one-way ANOVAs, descriptive analyses of the demographic and diagnostic groups are presented first to address the initial research aim of this dissertation. Results of calibration and validation factor analyses, using a randomized approximate 50/50 split of the combined WAIS-III and WAIS-IV dataset, are described regarding the first three hypotheses. The results of structural equation modeling that examines the fourth hypothesis are presented. Discussion, including implications and limitations of the findings, as well as suggestions for future research, are presented in Chapter Five.

Two variations of the two initial datasets were ultimately used for this study: Congruent with the initial research aim, the WAIS-III and WAIS-IV datasets were combined into one large dataset which was used to describe the participants with respect to the demographic and diagnostic variables. This combined dataset was then divided using a randomization procedure to provide independent datasets for calibration and 
validation purposes to test the hypothesized factor models. The WAIS-III and WAIS-IV datasets were combined also because dividing the WAIS-IV dataset alone into two datasets would have reduced the sample sizes for the calibration and validation studies, thereby providing an inadequate sample size to test the many parameters of the hypothesized models (Fabrigar, 1999; MacCallum, Widaman, Zhang, \& Hong, 1999). In the initial descriptive information provided in Tables $13-27$, however, information from all three datasets is provided to show the differences among the datasets. Although the WAIS-III and WAIS-IV did show some differences in variable means, the differences did not preclude the ability to combine the datasets to provide more robust factor analyses.

\section{Research Aim - Descriptive Analyses}

For the WAIS-III, WAIS-IV, and combined datasets, means, standard errors of the means, standard deviations, skew, and kurtosis of all measures are presented in Tables 13 - 27. Tables 13,14 , and 15 provide descriptive information regarding the WAIS-III calibration dataset with respect to the WAIS-III and WJ III cognitive and achievement variables. Tables 16,17 , and 18 provide similar information with respect to the WAISIV calibration dataset. Tables 19 and 20 describe the CAARS self- and other-rating scales for each calibration dataset. Information regarding the BDI-II and SCL-90-R in the WAIS-III and WAIS-IV calibration datasets is provided in Tables 21 and 22. Tables $23-27$ provide similar information for the combined WAIS-III/IV calibration dataset.

With the exception of the Planning subtest of the WJ III COG, score distributions from all three datasets appear to be relatively normal, with skew and kurtosis between 0 and 1 for most variables (Burdenski, 2000). As the main exception to this normality, 
Planning demonstrated a somewhat skewed $(2.47,2.20,2.32)$ and leptokurtic $(6.72,3.91$, 5.49) distribution in all three exploratory datasets. Another noteworthy finding upon examination of the dataset descriptive statistics is the discrepancy between the WAISIII/IV full-scale IQ (FSIQ) and the WJ III COG General Intellectual Ability (GIA) score on all three datasets. A paired-samples t-test indicated that the combined database WAIS mean score of $113.75(\mathrm{SD}=13.47)$ significantly exceeded the WJ III mean score of 104.24 $(\mathrm{SD}=12.24)$ by a full 9.5 points, $t(1138)=37.75, p<.001$.

As noted in Chapters I and II, one research aim of this study is to describe the students undergoing evaluation at the disabilities resource center. The age of participants ranged from 16.97 to 57.05 years $(M=27.27, S D=5.28)$. Age was non-normally distributed, with a skew of $2.46(S E=0.08)$ and kurtosis of $8.22(S E=0.16)$. As proposed, the data were also explored with respect to students' gender, ethnicity, diagnostic status, and whether they had requested a non-primary language substitution. 
Table 13

Means, Standard Errors, Standard Deviations, Skew and Kurtosis for WAIS-III FSIQ and Subtests of the WAIS-III Calibration Dataset

\begin{tabular}{lcccccc}
\hline FSIQ*/Subtest** & $n$ & $\mathrm{M}$ & $\mathrm{SE}$ & $\mathrm{SD}$ & Skew & Kurtosis \\
\hline FSIQ & 454 & 114.49 & 0.63 & 13.32 & -0.27 & 0.63 \\
Vocabulary & 454 & 13.32 & 0.14 & 2.93 & -0.35 & 0.28 \\
Similarities & 454 & 12.25 & 0.14 & 2.97 & 0.08 & -0.62 \\
Arithmetic & 454 & 12.13 & 0.12 & 2.60 & -0.38 & 0.47 \\
Digit Span & 454 & 11.22 & 0.13 & 2.87 & 0.46 & -0.33 \\
Information & 454 & 12.85 & 0.12 & 2.62 & -0.61 & 0.43 \\
Comprehension & 454 & 12.80 & 0.13 & 2.78 & -0.01 & 0.06 \\
Letter-Number Sequencing & 454 & 11.49 & 0.14 & 2.92 & 0.39 & -0.42 \\
Picture Completion & 454 & 10.41 & 0.15 & 3.13 & 0.03 & -0.52 \\
Digit Symbol & 454 & 10.45 & 0.13 & 2.83 & 0.25 & -0.20 \\
Block Design & 454 & 12.29 & 0.14 & 3.05 & -0.01 & -0.23 \\
Matrix Reasoning & 454 & 13.25 & 0.11 & 2.38 & -0.37 & -0.24 \\
Picture Arrangement & 454 & 11.57 & 0.13 & 2.76 & 0.20 & -0.41 \\
Symbol Search & 454 & 11.29 & 0.13 & 2.86 & 0.32 & 0.35 \\
\hline Note. WAIS-III Wech & & & & & &
\end{tabular}

Note. WAIS-III $=$ Wechsler Adult Intelligence Scale - Third Edition. FSIQ $=$ FullScale Intelligence Quotient. $\mathrm{M}=$ Mean. $\mathrm{SE}=$ Standard Error of the Mean. SD = Standard Deviation. *WAIS-III FSIQ has a Standard Score M = 100, SD = 15 . **Subtests have an Index Score $\mathrm{M}=10, \mathrm{SD}=3$. 
Table 14

Means, Standard Errors, Standard Deviations, Skew and Kurtosis for WJ III Cognitive GIA and Subtests of the WAIS-III Calibration Dataset

\begin{tabular}{lcccccc}
\hline GIA/Subtest* & $n$ & $\mathrm{M}$ & $\mathrm{SE}$ & $\mathrm{SD}$ & Skew & Kurtosis \\
\hline GIA & 451 & 104.57 & 0.57 & 12.17 & 0.18 & 0.57 \\
Verbal Comprehension & 396 & 103.85 & 0.62 & 12.27 & -0.28 & 0.96 \\
Visual-Auditory Learning & 449 & 100.59 & 0.83 & 17.55 & -0.09 & 0.80 \\
Spatial Relations & 394 & 106.85 & 0.52 & 10.33 & 0.11 & -0.01 \\
Sound Blending & 448 & 105.24 & 0.62 & 13.08 & -0.34 & 0.98 \\
Concept Formation & 448 & 107.12 & 0.57 & 11.99 & -0.20 & -0.11 \\
Visual Matching & 449 & 101.99 & 0.73 & 15.52 & 0.11 & 1.54 \\
Numbers Reversed & 362 & 104.73 & 0.80 & 15.22 & -0.03 & -0.06 \\
Incomplete Words & 446 & 107.05 & 0.68 & 14.44 & 0.12 & 1.30 \\
Retrieval Fluency & 394 & 98.60 & 0.51 & 10.18 & 0.03 & 0.43 \\
Picture Recognition & 445 & 102.96 & 0.57 & 12.12 & 0.41 & 1.36 \\
Analysis Synthesis & 449 & 109.55 & 0.64 & 13.48 & 0.17 & 0.32 \\
Decision Speed & 394 & 101.97 & 0.79 & 15.65 & 0.09 & 0.19 \\
Memory for Words & 448 & 103.45 & 0.65 & 13.66 & 0.03 & 0.81 \\
Rapid Picture Naming & 388 & 97.34 & 0.83 & 16.33 & 0.16 & 1.07 \\
Planning & 393 & 112.75 & 1.11 & 22.06 & 2.47 & 6.72 \\
Pair Cancellation & 393 & 100.56 & 0.71 & 14.01 & -0.21 & 0.27 \\
\hline Note. WJ & & & & & 0.16 \\
\hline
\end{tabular}

Note. WJ III = Woodcock-Johnson Tests of Cognitive Abilities - Third Edition. GIA = General Intellectual Ability. $\mathrm{M}=$ Mean. $\mathrm{SE}=$ Standard Error of the Mean. SD = Standard Deviation. *WJ III Cognitive GIA and subtests have a Standard Score M= $100, \mathrm{SD}=15$. 
Table 15

Means, Standard Errors, Standard Deviations, Skew and Kurtosis for WJ III Achievement Subtests of the WAIS-III Calibration Dataset

\begin{tabular}{lcccccc}
\hline Subtest* & $n$ & $\mathrm{M}$ & $\mathrm{SE}$ & $\mathrm{SD}$ & Skew & Kurtosis \\
\hline Letter-Word Identification & 456 & 104.82 & 0.53 & 11.26 & 0.70 & 2.41 \\
Reading Fluency & 396 & 102.14 & 0.75 & 14.83 & 0.37 & 0.10 \\
Calculation & 449 & 111.34 & 0.70 & 14.77 & 0.00 & -0.11 \\
Passage Comprehension & 455 & 105.68 & 0.49 & 10.55 & 0.03 & 0.47 \\
Math Fluency & 396 & 100.02 & 0.66 & 13.12 & 0.05 & 0.08 \\
Spelling & 398 & 107.19 & 0.51 & 10.13 & 0.09 & 0.52 \\
Writing Fluency & 445 & 106.59 & 0.68 & 14.45 & 0.07 & 1.32 \\
Applied Problems & 454 & 107.93 & 0.62 & 13.30 & 0.54 & 0.17 \\
Writing Samples & 456 & 109.37 & 0.77 & 16.55 & 0.59 & 2.05 \\
Word Attack & 439 & 101.59 & 0.52 & 10.99 & 0.09 & 0.95
\end{tabular}

Note. WJ III Achievement $=$ Woodcock-Johnson Tests of Achievement - Third

Edition. WAIS-III = Wechsler Adult Intelligence Scale - Third Edition. M = Mean. $\mathrm{SE}=$ Standard Error of the Mean. SD $=$ Standard Deviation. *WJ III Achievement subtests have a Standard Score $\mathrm{M}=100, \mathrm{SD}=15$. 
Table 16

Means, Standard Errors, Standard Deviations, Skew and Kurtosis for Subtests of the WAIS-IV Calibration Dataset

\begin{tabular}{|c|c|c|c|c|c|c|}
\hline FSIQ*/Subtest** & $n$ & M & SE & SD & Skew & Kurtosis \\
\hline FSIQ & 120 & 109.66 & 1.30 & 14.21 & -0.04 & -0.82 \\
\hline Similarities & 120 & 11.78 & 0.30 & 3.29 & -0.31 & -0.37 \\
\hline Vocabulary & 120 & 12.43 & 0.30 & 3.25 & -0.27 & -0.01 \\
\hline Information & 120 & 12.17 & 0.26 & 2.86 & -0.16 & -0.67 \\
\hline Comprehension & 120 & 11.91 & 0.27 & 2.98 & 0.03 & -0.58 \\
\hline Block Design & 120 & 10.82 & 0.32 & 3.53 & 0.03 & -0.50 \\
\hline Matrix Reasoning & 120 & 12.06 & 0.26 & 2.87 & -0.38 & 0.15 \\
\hline Visual Puzzles & 119 & 11.06 & 0.27 & 3.00 & 0.07 & -0.80 \\
\hline Figure Weights & 118 & 12.71 & 0.28 & 3.04 & -0.27 & -0.22 \\
\hline Picture Completion & 119 & 9.43 & 0.28 & 3.03 & -0.04 & -0.58 \\
\hline Digit Span & 120 & 11.07 & 0.26 & 2.87 & 0.14 & -0.29 \\
\hline Arithmetic & 120 & 11.88 & 0.27 & 2.97 & -0.22 & -0.23 \\
\hline Letter-Number Sequencing & 120 & 11.52 & 0.31 & 3.35 & 0.82 & 0.01 \\
\hline Symbol Search & 120 & 10.13 & 0.30 & 3.30 & 0.21 & 0.20 \\
\hline Coding & 120 & 10.52 & 0.27 & 3.01 & 0.16 & -0.53 \\
\hline Cancellation & 35 & 10.11 & 0.55 & 3.27 & 0.56 & -0.42 \\
\hline
\end{tabular}

Note. WAIS-IV $=$ Wechsler Adult Intelligence Scale - Fourth Edition. M = Mean. SE

$=$ Standard Error of the Mean. SD $=$ Standard Deviation. FSIQ $=$ Full Scale

Intelligence Quotient. *WAIS-IV FSIQ has a Standard Score $\mathrm{M}=100, \mathrm{SD}=15$.

$* *$ Subtests have an Index Score $\mathrm{M}=10, \mathrm{SD}=3$. 
Table 17

Means, Standard Errors, Standard Deviations, Skew and Kurtosis for WJ III Cognitive GIA and Subtests of the WAIS-IV Calibration Dataset

\begin{tabular}{|c|c|c|c|c|c|c|}
\hline GIA/Subtest* & $n$ & $\mathrm{M}$ & $\mathrm{SE}$ & SD & Skew & Kurtosis \\
\hline GIA & 117 & 103.14 & 1.17 & 12.63 & -0.17 & -0.82 \\
\hline Verbal Comprehension & 117 & 104.88 & 1.12 & 12.10 & -0.33 & -0.47 \\
\hline Visual-Auditory Learning & 118 & 96.87 & 1.92 & 20.86 & -0.44 & 0.36 \\
\hline Spatial Relations & 118 & 104.28 & 1.01 & 10.99 & -0.50 & -0.21 \\
\hline Sound Blending & 118 & 102.98 & 1.18 & 12.87 & -0.17 & 0.11 \\
\hline Concept Formation & 118 & 109.30 & 1.07 & 11.60 & -0.51 & -0.20 \\
\hline Visual Matching & 118 & 100.02 & 1.46 & 15.84 & 0.15 & 1.09 \\
\hline Numbers Reversed & 118 & 105.67 & 1.26 & 13.69 & -0.06 & -0.37 \\
\hline Incomplete Words & 116 & 104.91 & 1.32 & 14.17 & 0.03 & -0.28 \\
\hline Retrieval Fluency & 118 & 95.08 & 1.06 & 11.51 & -0.11 & 0.14 \\
\hline Picture Recognition & 118 & 100.94 & 1.00 & 10.82 & 0.50 & -0.10 \\
\hline Analysis-Synthesis & 118 & 112.84 & 1.29 & 14.06 & -0.24 & 0.06 \\
\hline Decision Speed & 118 & 101.70 & 1.53 & 16.63 & 0.21 & 0.37 \\
\hline Memory for Words & 117 & 100.79 & 1.18 & 12.80 & -0.20 & -0.38 \\
\hline Rapid Picture Naming & 118 & 94.10 & 1.67 & 18.14 & -0.02 & -0.96 \\
\hline Planning & 117 & 113.91 & 2.58 & 27.96 & 2.20 & 3.91 \\
\hline Pair Cancellation & 117 & 96.03 & 1.47 & 15.85 & 0.21 & -0.47 \\
\hline
\end{tabular}

Note. WJ III Cognitive $=$ Woodcock-Johnson Tests of Cognitive Abilities - Third Edition. WAIS-IV $=$ Wechsler Adult Intelligence Scale - Fourth Edition. GIA = General Intellectual Ability. $\mathrm{M}=$ Mean. $\mathrm{SE}=$ Standard Error of the Mean. SD $=$ Standard Deviation. *WJ III Cognitive GIA and subtests have a Standard Score $\mathrm{M}=100, \mathrm{SD}=15$. 
Table 18

Means, Standard Errors, Standard Deviations, Skew and Kurtosis for WJ III Achievement Subtests of the WAIS-IV Calibration Dataset

\begin{tabular}{lcccccc}
\hline Subtest* & $n$ & $\mathrm{M}$ & $\mathrm{SE}$ & $\mathrm{SD}$ & Skew & Kurtosis \\
\hline Letter-Word Identification & 118 & 104.30 & 0.71 & 7.70 & -0.11 & -0.57 \\
Reading Fluency & 118 & 101.95 & 1.27 & 13.78 & 0.35 & 0.33 \\
Calculation & 118 & 111.74 & 1.34 & 14.51 & -0.29 & -0.15 \\
Passage Comprehension & 118 & 105.81 & 0.96 & 10.41 & 0.11 & 0.23 \\
Math Fluency & 118 & 101.14 & 1.35 & 14.71 & 0.06 & 0.39 \\
Spelling & 118 & 108.22 & 0.79 & 8.54 & -0.39 & 0.19 \\
Writing Fluency & 118 & 107.00 & 1.31 & 14.23 & 0.56 & 0.39 \\
Applied Problems & 118 & 108.67 & 1.11 & 12.06 & -0.75 & 0.32 \\
Writing Samples & 118 & 108.18 & 1.06 & 11.55 & 0.41 & 1.39 \\
Word Attack & 117 & 103.74 & 0.92 & 9.91 & -0.38 & 0.11
\end{tabular}

Note. WJ III Achievement $=$ Woodcock-Johnson Tests of Achievement - Third

Edition. WAIS-IV $=$ Wechsler Adult Intelligence Scale - Fourth Edition. $M=$ Mean. $\mathrm{SE}=$ Standard Error of the Mean. SD = Standard Deviation. *WJ III Achievement subtests have a Standard Score $\mathrm{M}=100, \mathrm{SD}=15$. 
Table 19

Means, Standard Errors, Standard Deviations, Skew and Kurtosis for FSIQ and Subtests of the Combined WAIS-III/IV Calibration Dataset

\begin{tabular}{lrrrrrr}
\hline FSIQ*/Subtest** & $n$ & \multicolumn{1}{c}{$\mathrm{M}$} & $\mathrm{SE}$ & $\mathrm{SD}$ & Skew & Kurtosis \\
\hline FSIQ & 573 & 113.75 & 0.56 & 13.47 & -0.01 & -0.34 \\
Vocabulary & 573 & 13.12 & 0.13 & 3.03 & -0.35 & 0.19 \\
Similarities & 573 & 12.18 & 0.13 & 3.08 & -0.05 & -0.38 \\
Arithmetic & 573 & 12.07 & 0.11 & 2.65 & -0.37 & 0.39 \\
Digit Span & 573 & 11.16 & 0.12 & 2.91 & 0.38 & -0.27 \\
$\quad$ D.S. Forward (Raw) & 469 & 10.75 & 0.10 & 2.22 & 0.00 & -0.50 \\
D.S. Backward (Raw) & 469 & 8.51 & 0.12 & 2.53 & 0.27 & -0.29 \\
Information & 573 & 12.74 & 0.11 & 2.72 & -0.48 & 0.11 \\
Comprehension & 572 & 12.65 & 0.12 & 2.83 & 0.02 & -0.05 \\
Letter-Number Sequencing & 573 & 11.42 & 0.13 & 3.04 & 0.44 & -0.26 \\
Picture Completion & 573 & 10.27 & 0.13 & 3.11 & 0.07 & -0.48 \\
Coding (Digit Symbol) & 573 & 10.57 & 0.12 & 2.86 & 0.22 & -0.32 \\
Block Design & 573 & 12.08 & 0.13 & 3.13 & -0.03 & -0.24 \\
Matrix Reasoning & 573 & 13.03 & 0.10 & 2.50 & -0.37 & -0.15 \\
Picture Arrangement & 454 & 11.57 & 0.13 & 2.76 & 0.20 & -0.41 \\
Visual Puzzles & 117 & 11.42 & 0.27 & 2.87 & 0.03 & -0.89 \\
Figure Weights & 116 & 12.67 & 0.27 & 2.92 & -0.19 & -0.23 \\
Symbol Search & 573 & 11.09 & 0.12 & 2.92 & 0.21 & 0.37 \\
Cancellation & 33 & 10.61 & 0.55 & 3.18 & 0.47 & -0.72 \\
\hline Note. WAIS-II/IV W & & & & & & \\
\hline
\end{tabular}

Note. WAIS-III/IV = Wechsler Adult Intelligence Scale - Third/Fourth Editions. FSIQ $=$ Full-Scale Intelligence Quotient. $\mathrm{M}=$ Mean $. \mathrm{SE}=$ Standard Error of the Mean. $\mathrm{SD}=$ Standard Deviation. *WAIS-III/IV FSIQs have a Standard Score M = $100, \mathrm{SD}=15 . * *$ Subtests have an Index Score $\mathrm{M}=10, \mathrm{SD}=3$. 
Table 20

Means, Standard Errors, Standard Deviations, Skew and Kurtosis for WJ III Cognitive GIA and Subtests of the Combined WAIS-III/IV Calibration Dataset

\begin{tabular}{lcccccc}
\hline GIA/Subtest* & \multicolumn{1}{c}{$n$} & $\mathrm{M}$ & $\mathrm{SE}$ & $\mathrm{SD}$ & Skew & Kurtosis \\
\hline GIA & 566 & 104.24 & 0.51 & 12.24 & -0.04 & 0.02 \\
Verbal Comprehension & 511 & 103.98 & 0.55 & 12.40 & -0.31 & 0.65 \\
Visual-Auditory Learning & 564 & 100.00 & 0.75 & 17.89 & -0.18 & 0.81 \\
Spatial Relations & 509 & 106.05 & 0.50 & 11.36 & -1.17 & 8.41 \\
Sound Blending & 563 & 104.57 & 0.55 & 13.05 & -0.29 & 0.81 \\
Concept Formation & 563 & 107.67 & 0.50 & 11.90 & -0.24 & -0.25 \\
Visual Matching & 564 & 102.10 & 0.64 & 15.31 & 0.07 & 1.44 \\
Numbers Reversed & 477 & 104.70 & 0.69 & 15.06 & -0.11 & 0.03 \\
Incomplete Words & 560 & 106.62 & 0.61 & 14.44 & 0.06 & 1.14 \\
Retrieval Fluency & 509 & 97.99 & 0.47 & 10.70 & -0.16 & 0.42 \\
Picture Recognition & 560 & 102.44 & 0.50 & 11.84 & 0.43 & 1.24 \\
Analysis Synthesis & 564 & 110.01 & 0.57 & 13.47 & 0.06 & 0.28 \\
Decision Speed & 509 & 102.17 & 0.69 & 15.59 & 0.14 & 0.30 \\
Memory for Words & 562 & 102.26 & 0.41 & 13.89 & -0.22 & 1.27 \\
Rapid Picture Naming & 503 & 97.37 & 0.76 & 16.98 & 0.08 & 0.52 \\
Planning & 506 & 113.21 & 1.05 & 23.60 & 2.32 & 5.49 \\
Pair Cancellation & 507 & 99.93 & 0.62 & 14.04 & -0.16 & 0.14 \\
\hline Note. WJ III Cognitive Wo0 & & & & & & \\
\hline
\end{tabular}

Note. WJ III Cognitive $=$ Woodcock-Johnson Tests of Cognitive Abilities - Third Edition. WAIS-III/IV = Wechsler Adult Intelligence Scale - Third/Fourth Editions. $\mathrm{GIA}=$ General Intellectual Ability. $\mathrm{M}=$ Mean. $\mathrm{SE}=$ Standard Error of the Mean . $\mathrm{SD}=$ Standard Deviation. *WJ III GIA and subtests have a Standard Score $\mathrm{M}=100$, $\mathrm{SD}=15$. 
Table 21

Means, Standard Errors, Standard Deviations, Skew and Kurtosis for the WJ III Achievement Tests of the WAIS-III/IV Combined Calibration Dataset

\begin{tabular}{lcccccr}
\hline Subtest* & $n$ & $\mathrm{M}$ & $\mathrm{SE}$ & $\mathrm{SD}$ & Skew & Kurtosis \\
\hline Letter-Word Identification & 572 & 104.72 & 0.45 & 10.86 & 0.72 & 3.11 \\
Reading Fluency & 512 & 102.18 & 0.64 & 14.54 & 0.33 & 0.18 \\
Calculation & 565 & 111.48 & 0.62 & 14.75 & -0.10 & 0.12 \\
Passage Comprehension & 512 & 100.27 & 0.59 & 13.27 & -0.07 & 0.00 \\
Math Fluency & 514 & 107.36 & 0.44 & 9.94 & -0.04 & 0.62 \\
Spelling & 561 & 106.85 & 0.61 & 14.38 & 0.06 & 1.02 \\
Writing Fluency & 571 & 105.60 & 0.44 & 10.50 & 0.04 & 0.41 \\
Applied Problems & 570 & 108.04 & 0.55 & 13.04 & 0.44 & 0.06 \\
Writing Samples & 572 & 108.88 & 0.66 & 15.73 & 0.57 & 2.36 \\
Word Attack & 555 & 101.97 & 0.46 & 10.85 & -0.02 & 0.78
\end{tabular}

Note. WJ III Achievement $=$ Woodcock-Johnson Tests of Achievement - Third Edition. WAIS-III/IV = Wechsler Adult Intelligence Scale - Third/Fourth Editions. $\mathrm{M}=$ Mean. $\mathrm{SE}=$ Standard Error of the Mean. SD = Standard Deviation. *WJ III Achievement subtests have a Standard Score $\mathrm{M}=100, \mathrm{SD}=15$. 
Table 22

Means, Standard Errors, Standard Deviations, Skew and Kurtosis for Rating Scale Items of the Self-and Other-Rated CAARS of the WAIS-III Calibration Dataset

\begin{tabular}{|c|c|c|c|c|c|c|}
\hline Scale* & $n$ & M & $\mathrm{SE}$ & SD & Skew & Kurtosis \\
\hline $\begin{array}{l}\text { S-A - Inattention/Memory } \\
\text { Problems }\end{array}$ & 458 & 64.06 & 0.61 & 12.95 & -0.38 & -0.53 \\
\hline $\begin{array}{c}\text { S-B - Hyperactivity/ } \\
\text { Restlessness }\end{array}$ & 458 & 54.95 & 0.56 & 11.94 & 0.09 & -0.72 \\
\hline $\begin{array}{l}\mathrm{S}-\mathrm{C}-\text { Impulsivity/ } \\
\text { Emotional Lability }\end{array}$ & 458 & 52.78 & 0.54 & 11.48 & 0.32 & -0.37 \\
\hline $\begin{array}{l}\text { S-D - Problems with Self- } \\
\text { Concept }\end{array}$ & 458 & 55.36 & 0.56 & 11.89 & -0.10 & -0.69 \\
\hline $\begin{array}{l}\text { S-E - DSM-IV Inattentive } \\
\text { Symptoms }\end{array}$ & 458 & 72.96 & 0.69 & 14.84 & -0.70 & -0.46 \\
\hline $\begin{array}{c}\text { S-F - DSM-IV Hyperactive- } \\
\text { Impulsive Symptoms }\end{array}$ & 457 & 57.51 & 0.70 & 15.07 & 0.24 & -0.83 \\
\hline $\begin{array}{l}\text { S-G - DSM-IV ADHD } \\
\text { Symptoms Total }\end{array}$ & 457 & 68.62 & 0.71 & 15.27 & -0.46 & -0.67 \\
\hline S-H - ADHD Index & 457 & 59.30 & 0.50 & 10.66 & -0.35 & -0.04 \\
\hline $\begin{array}{l}\text { O-A - Inattention/ Memory } \\
\text { Problems }\end{array}$ & 262 & 61.92 & 0.81 & 13.11 & -0.16 & -0.97 \\
\hline $\begin{array}{c}\text { O-B - Hyperactivity/ } \\
\text { Restlessness }\end{array}$ & 262 & 54.12 & 0.78 & 12.56 & 0.29 & -0.93 \\
\hline $\begin{array}{l}\text { O-C - Impulsivity/ } \\
\text { Emotional Lability }\end{array}$ & 262 & 52.72 & 0.66 & 10.65 & 0.38 & -0.45 \\
\hline $\begin{array}{l}\text { O-D - Problems with Self- } \\
\text { Concept }\end{array}$ & 262 & 55.10 & 0.71 & 11.54 & 0.30 & -0.96 \\
\hline $\begin{array}{l}\text { O-E - DSM-IV Inattentive } \\
\text { Symptoms }\end{array}$ & 262 & 61.28 & 0.74 & 11.90 & -0.22 & -0.79 \\
\hline $\begin{array}{c}\text { O-F - DSM-IV Hyperactive- } \\
\text { Impulsive Symptoms }\end{array}$ & 262 & 54.72 & 0.80 & 12.97 & 0.49 & -0.56 \\
\hline $\begin{array}{l}\text { O-G - DSM-IV ADHD } \\
\text { Symptoms Total }\end{array}$ & 262 & 59.53 & 0.75 & 12.20 & 0.08 & -0.64 \\
\hline $\mathrm{O}-\mathrm{H}$ - ADHD Index & 262 & 58.62 & 0.70 & 11.30 & 0.00 & -0.59 \\
\hline
\end{tabular}


Table 23

Means, Standard Errors, Standard Deviations, Skew and Kurtosis for Rating Scale Items of the Self-and Other-Rated CAARS from the WAIS-IV Calibration Dataset

\begin{tabular}{|c|c|c|c|c|c|c|}
\hline Scale* & $n$ & M & $\mathrm{SE}$ & SD & Skew & Kurtosis \\
\hline $\begin{array}{c}\text { S-A - Inattention/Memory } \\
\text { Problems }\end{array}$ & 120.00 & 65.15 & 1.22 & 13.40 & -0.50 & -0.57 \\
\hline $\begin{array}{c}\text { S-B - Hyperactivity/ } \\
\text { Restlessness }\end{array}$ & 120.00 & 56.08 & 1.12 & 12.26 & -0.18 & -0.96 \\
\hline $\begin{array}{l}\text { S-C - Impulsivity/ } \\
\text { Emotional Lability }\end{array}$ & 120.00 & 53.02 & 1.21 & 13.27 & 0.33 & -0.91 \\
\hline $\begin{array}{l}\text { S-D - Problems with Self- } \\
\text { Concept }\end{array}$ & 120.00 & 56.16 & 1.18 & 12.98 & 0.09 & -1.25 \\
\hline $\begin{array}{l}\text { S-E - DSM-IV Inattentive } \\
\text { Symptoms }\end{array}$ & 120.00 & 74.71 & 1.34 & 14.71 & -0.87 & -0.14 \\
\hline $\begin{array}{c}\text { S-F - DSM-IV Hyperactive- } \\
\text { Impulsive Symptoms }\end{array}$ & 120.00 & 59.02 & 1.43 & 15.67 & 0.02 & -1.03 \\
\hline $\begin{array}{r}\text { S-G - DSM-IV ADHD } \\
\text { Symptoms Total }\end{array}$ & 120.00 & 70.03 & 1.46 & 15.99 & -0.56 & -0.57 \\
\hline S-H - ADHD Index & 120.00 & 60.17 & 1.13 & 12.41 & -0.20 & -0.71 \\
\hline $\begin{array}{l}\text { O-A - Inattention/ Memory } \\
\text { Problems }\end{array}$ & 98.00 & 62.18 & 1.53 & 15.12 & -0.30 & -0.96 \\
\hline $\begin{array}{c}\text { O-B - Hyperactivity/ } \\
\text { Restlessness }\end{array}$ & 98.00 & 56.85 & 1.34 & 13.22 & 0.19 & -1.09 \\
\hline $\begin{array}{l}\text { O-C - Impulsivity/ } \\
\text { Emotional Lability }\end{array}$ & 98.00 & 52.10 & 1.14 & 11.25 & 0.39 & -0.43 \\
\hline $\begin{array}{l}\text { O-D - Problems with Self- } \\
\text { Concept }\end{array}$ & 98.00 & 54.28 & 1.28 & 12.66 & 0.29 & -1.17 \\
\hline $\begin{array}{l}\text { O-E - DSM-IV Inattentive } \\
\text { Symptoms }\end{array}$ & 98.00 & 61.69 & 1.38 & 13.64 & -0.27 & -0.91 \\
\hline $\begin{aligned} \text { O-F - DSM-IV } & \\
& \text { Hyperactive- } \\
& \text { Impulsive Symptoms }\end{aligned}$ & 98.00 & 57.24 & 1.42 & 14.07 & 0.25 & -1.05 \\
\hline $\begin{array}{l}\text { O-G - DSM-IV ADHD } \\
\text { Symptoms Total }\end{array}$ & 98.00 & 60.70 & 1.40 & 13.88 & -0.13 & -0.83 \\
\hline $\mathrm{O}-\mathrm{H}$ - ADHD Index & 98.00 & 59.22 & 1.32 & 13.06 & -0.19 & -0.86 \\
\hline
\end{tabular}


Table 24

Means, Standard Errors, Standard Deviations, Skew and Kurtosis for Rating Scale Items of the Self-and Other-Rated CAARS from the WAIS-III/IV Combined Calibration Dataset

\begin{tabular}{|c|c|c|c|c|c|c|}
\hline Scale* & $n$ & M & SE & SD & Skew & Kurtosis \\
\hline $\begin{array}{l}\text { S-A - Inattention/Memory } \\
\text { Problems }\end{array}$ & 574 & 64.21 & 0.55 & 13.08 & -0.39 & -0.52 \\
\hline $\begin{array}{c}\text { S-B - Hyperactivity/ } \\
\text { Restlessness }\end{array}$ & 574 & 55.30 & 0.50 & 12.03 & 0.03 & -0.78 \\
\hline $\begin{array}{l}\text { S-C - Impulsivity/ Emotional } \\
\text { Lability }\end{array}$ & 574 & 52.92 & 0.49 & 11.69 & 0.34 & -0.42 \\
\hline $\begin{array}{l}\text { S-D - Problems with Self- } \\
\text { Concept }\end{array}$ & 574 & 55.55 & 0.50 & 12.03 & -0.08 & -0.83 \\
\hline $\begin{array}{l}\text { S-E - DSM-IV Inattentive } \\
\text { Symptoms }\end{array}$ & 574 & 73.22 & 0.62 & 14.75 & -0.72 & -0.41 \\
\hline $\begin{array}{c}\text { S-F - DSM-IV Hyperactive- } \\
\text { Impulsive Symptoms }\end{array}$ & 573 & 57.92 & 0.63 & 14.98 & 0.17 & -0.83 \\
\hline $\begin{array}{l}\text { S-G - DSM-IV ADHD } \\
\text { Symptoms Total }\end{array}$ & 573 & 69.01 & 0.64 & 15.22 & -0.50 & -0.58 \\
\hline S-H - ADHD Index & 573 & 59.57 & 0.46 & 10.93 & -0.35 & -0.14 \\
\hline $\begin{array}{l}\text { O-A - Inattention/ Memory } \\
\text { Problems }\end{array}$ & 354 & 62.10 & 0.72 & 13.53 & -0.24 & -0.91 \\
\hline $\begin{array}{c}\text { O-B - Hyperactivity/ } \\
\text { Restlessness }\end{array}$ & 354 & 54.85 & 0.69 & 13.03 & 0.27 & -1.02 \\
\hline $\begin{array}{l}\text { O-C - Impulsivity/ } \\
\text { Emotional Lability }\end{array}$ & 354 & 52.71 & 0.57 & 10.67 & 0.34 & -0.43 \\
\hline $\begin{array}{l}\text { O-D - Problems with Self- } \\
\text { Concept }\end{array}$ & 354 & 54.94 & 0.62 & 11.72 & 0.26 & -0.97 \\
\hline $\begin{array}{l}\text { O-E - DSM-IV Inattentive } \\
\text { Symptoms }\end{array}$ & 354 & 61.31 & 0.66 & 12.33 & -0.25 & -0.77 \\
\hline $\begin{array}{c}\text { O-F - DSM-IV Hyperactive- } \\
\text { Impulsive Symptoms }\end{array}$ & 354 & 55.27 & 0.70 & 13.12 & 0.44 & -0.63 \\
\hline $\begin{array}{l}\text { O-G - DSM-IV ADHD } \\
\text { Symptoms Total }\end{array}$ & 354 & 59.75 & 0.66 & 12.51 & 0.02 & -0.66 \\
\hline O-H - ADHD Index & 354 & 58.88 & 0.62 & 11.71 & -0.07 & -0.62 \\
\hline \multicolumn{7}{|c|}{$\begin{array}{l}\text { Note. CAARS = Conners Adult ADHD Rating Scale. WAIS-III/IV = Wechsler } \\
\text { Adult Intelligence Scale }- \text { Third/Fourth Editions. } \mathrm{M}=\mathrm{Mean} . \mathrm{SE}=\text { Standard Error of } \\
\text { the Mean. SD }=\text { Standard Deviation. } \mathrm{S}=\text { Self-Rated. } \mathrm{O}=\text { Other Rated. CAARS } \\
\text { Rating Scales have a T-score } \mathrm{M}=50, \mathrm{SD}=10 \mathrm{CAARS}=\text { Conners Adult ADHD } \\
\text { Rating Scale. SE }=\text { Standard Error of the Mean. SD = Standard Deviation. } \\
\text { *CAARS Rating Scales have a T-score } \mathrm{M}=50, \mathrm{SD}=10 .\end{array}$} \\
\hline
\end{tabular}


Table 25

Means, Standard Errors, Standard Deviations, Skew and Kurtosis for the BDI-II Total Score and SCL-90-R GSI of the WAIS-III Calibration Dataset

\begin{tabular}{lcccccc}
\hline Scale* & $n$ & $\mathrm{M}$ & $\mathrm{SE}$ & $\mathrm{SD}$ & Skew & Kurtosis \\
\hline BDI-II Total Score & 433 & 13.76 & 0.49 & 10.17 & 0.86 & 0.31 \\
SCL-90-R GSI & 405 & 58.83 & 0.64 & 12.83 & -0.17 & -0.93 \\
\hline
\end{tabular}

Note. BDI-II = Beck Depression Inventory - Second Edition. SCL-90-R GSI = Symptom Checklist-90-Revised Global Severity Index. WAIS-III = Wechsler Adult Intelligence Scale - Third Edition. $\mathrm{M}=$ Mean. $\mathrm{SE}=$ Standard Error of the Mean. SD $=$ Standard Deviation. ${ }^{*}$ Cut score guidelines for the BDI-II Total Score: $0-13$ is considered minimal range, 14-19 is mild, 20-28 is moderate, and 29-63 is severe for symptoms of depression; the GSI has a T-score $\mathrm{M}=50, \mathrm{SD}=10$.

Table 26

Means, Standard Errors, Standard Deviations, Skew and Kurtosis for the BDI-II Total Score and SCL-90-R GSI of the WAIS-IV Calibration Dataset

\begin{tabular}{lcccrrc}
\hline Scale* & $n$ & M & SE & SD & Skew & Kurtosis \\
\hline BDI-II Total Score & 95 & 13.60 & 1.00 & 9.76 & 0.97 & 0.76 \\
SCL-90-R GSI & 92 & 60.74 & 1.05 & 10.10 & -0.28 & -0.28 \\
\hline
\end{tabular}

Note. BDI-II = Beck Depression Inventory - Second Edition. SCL-90-R GSI = Symptom Checklist-90-Revised Global Severity Index. WAIS-IV = Wechsler Adult Intelligence Scale - Fourth Edition. $\mathrm{M}=$ Mean. $\mathrm{SE}=$ Standard Error of the Mean. $\mathrm{SD}=$ Standard Deviation. ${ }^{*}$ Cut score guidelines for the BDI-II Total Score: $0-13$ is considered minimal range, 14-19 is mild, 20-28 is moderate, and 29-63 is severe for symptoms of depression; the GSI has a T-score $\mathrm{M}=50, \mathrm{SD}=10$.

Table 27

Means, Standard Errors, Standard Deviations, Skew and Kurtosis for BDI-II Total Score and SCL-90-R GSI of the WAIS-III/IV Combined Calibration Dataset

\begin{tabular}{lcccccc}
\hline Scale* & $n$ & $\mathrm{M}$ & $\mathrm{SE}$ & $\mathrm{SD}$ & Skew & Kurtosis \\
\hline BDI-II Total Score & 527 & 14.02 & 0.45 & 10.31 & 0.84 & 0.29 \\
SCL-90-R GSI & 494 & 59.18 & 0.57 & 12.66 & -0.21 & -0.88 \\
\hline
\end{tabular}

Note. BDI-II = Beck Depression Inventory - Second Edition. SCL-90-R GSI = Symptom Checklist-90-Revised Global Severity Index. $M=$ Mean. SE = Standard Error of the Mean. SD = Standard Deviation. *Cut score guidelines for the BDI-II Total score: $0-13$ is considered minimal range, $14-19$ is mild, 20-28 is moderate, and 29-63 is severe for symptoms of depression. **The GSI has a T-score $\mathrm{M}=50, \mathrm{SD}=10$. 


\section{Gender}

Tables 28 - 33 provide descriptive information for the combined dataset WAISIII/IV test scores, the WJ III Cognitive and Achievement test scores, the CAARS selfand other-rating scales, and the BDI-II and SCL-90-R GSI rating scale scores by gender. Independent samples $t$-tests indicated that men scored significantly higher than women on the WAIS-III/IV FSIQ, but not on the WJ III GIA. Men also performed significantly better than women on every test of quantitative reasoning, including Arithmetic, Calculation, Applied Problems, and Math Fluency. This last finding is interesting in that the women significantly bested the men in all other fluency and speed tasks, including Reading and Writing Fluency, Retrieval Fluency, Rapid Picture Naming, Coding, and Symbol Search. Although the women also outperformed the men in the auditory processing task of Sound Blending and the visual-spatial memory task of Picture Recognition, they fared more poorly against the men in the working-memory capacity tasks of Digit Span, Letter-Number Sequencing, and Numbers Reversed; the visualspatial processing tasks of Block Design, Picture Arrangement, and Planning; and the comprehension-knowledge tasks of Information and Verbal Comprehension.

Table 28

Means, Standard Errors, Standard Deviations, and t-test Results of the Combined WAIS-III/IV FSIQ and Subtests by Gender

\begin{tabular}{llccccccr}
\hline FSIQ*/Subtest** & Gender & $n$ & $\mathrm{M}$ & $\mathrm{SE}$ & $\mathrm{SD}$ & $t$ & $\mathrm{df}$ & $p$ \\
\hline FSIQ & Female & 531 & 111.41 & 0.54 & 12.40 & & & \\
& Male & 652 & 113.73 & 0.56 & 14.41 & -2.97 & 1177 & .003 \\
Vocabulary & Female & 531 & 13.02 & 0.12 & 2.87 & & & \\
& Male & 653 & 12.89 & 0.13 & 3.27 & 0.73 & 1176 & .465 \\
Similarities & Female & 531 & 12.05 & 0.12 & 2.84 & & & \\
& Male & 653 & 12.08 & 0.12 & 3.17 & -0.17 & 1171 & .865
\end{tabular}


Table 28

Means, Standard Errors, Standard Deviations, and t-test Results of the Combined WAIS-III/IV FSIQ and Subtests by Gender, continued

\begin{tabular}{|c|c|c|c|c|c|c|c|c|}
\hline FSIQ*/ Subtest** & Gender & $n$ & M & $\mathrm{SE}$ & SD & $t$ & $\mathrm{df}$ & $p$ \\
\hline \multirow{2}{*}{ Arithmetic } & Female & 531 & 11.23 & 0.11 & 2.58 & & & \\
\hline & Male & 653 & 12.52 & 0.11 & 2.71 & -8.35 & 1182 & $<.001$ \\
\hline \multirow[t]{2}{*}{ Digit Span } & Female & 531 & 10.70 & 0.12 & 2.75 & & & \\
\hline & Male & 653 & 11.20 & 0.12 & 3.05 & -2.93 & 1182 & $<.001$ \\
\hline \multirow[t]{2}{*}{ Information } & Female & 531 & 12.08 & 0.11 & 2.55 & & & \\
\hline & Male & 653 & 13.19 & 0.11 & 2.82 & -7.08 & 1182 & $<.001$ \\
\hline \multirow[t]{2}{*}{ Comprehension } & Female & 530 & 12.48 & 0.12 & 2.74 & & & \\
\hline & Male & 651 & 12.77 & 0.12 & 3.01 & -1.77 & 1164 & .080 \\
\hline \multirow{2}{*}{$\begin{array}{l}\text { Letter-Number } \\
\text { Sequencing }\end{array}$} & Female & 531 & 10.85 & 0.13 & 2.89 & & & \\
\hline & Male & 653 & 11.59 & 0.13 & 3.28 & -4.09 & 1174 & $<.001$ \\
\hline \multirow{2}{*}{$\begin{array}{l}\text { Picture } \\
\text { Completion }\end{array}$} & Female & 530 & 10.23 & 0.13 & 2.90 & & & \\
\hline & Male & 652 & 10.11 & 0.12 & 3.16 & 0.68 & 1163 & .499 \\
\hline \multirow[t]{2}{*}{ Coding } & Female & 531 & 11.09 & 0.12 & 2.81 & & & \\
\hline & Male & 653 & 9.95 & 0.11 & 2.87 & 6.87 & 1182 & $<.001$ \\
\hline \multirow[t]{2}{*}{ Block Design } & Female & 531 & 11.35 & 0.13 & 2.99 & & & \\
\hline & Male & 653 & 12.18 & 0.13 & 3.34 & -4.53 & 1171 & $<.001$ \\
\hline \multirow{2}{*}{$\begin{array}{l}\text { Matrix } \\
\text { Reasoning }\end{array}$} & Female & 531 & 12.69 & 0.11 & 2.50 & & & \\
\hline & Male & 652 & 12.93 & 0.11 & 2.72 & -1.56 & 1181 & .119 \\
\hline \multirow{2}{*}{$\begin{array}{l}\text { Picture } \\
\text { Arrangement }\end{array}$} & Female & 424 & 11.13 & 0.14 & 2.81 & & & \\
\hline & Male & 510 & 11.60 & 0.13 & 2.88 & -2.50 & 932 & .013 \\
\hline \multirow[t]{2}{*}{ Visual Puzzles } & Female & 105 & 11.21 & 0.27 & 2.73 & & & \\
\hline & Male & 142 & 10.88 & 0.25 & 2.98 & 0.89 & 245 & .375 \\
\hline \multirow[t]{2}{*}{ Figure Weights } & Female & 105 & 12.32 & 0.26 & 2.67 & & & \\
\hline & Male & 141 & 12.57 & 0.26 & 3.10 & -0.67 & 244 & .507 \\
\hline \multirow[t]{2}{*}{ Symbol Search } & Female & 531 & 11.29 & 0.13 & 3.02 & & & \\
\hline & Male & 652 & 10.68 & 0.12 & 2.96 & 3.52 & 1181 & $<.001$ \\
\hline \multirow[t]{2}{*}{ Cancellation } & Female & 39 & 9.90 & 0.51 & 3.20 & & & \\
\hline & Male & 28 & 9.21 & 0.54 & 2.86 & 0.90 & 65 & .371 \\
\hline
\end{tabular}

Note. WAIS-III/IV $=$ Wechsler Adult Intelligence Scale - Third/Fourth Editions. FSIQ = Full-Scale Intelligence Quotient. $\mathrm{M}=$ Mean. $\mathrm{SE}=$ Standard Error of the Mean. SD $=$ Standard Deviation. $\mathrm{df}=$ degrees of freedom. *WAIS-III/IV FSIQs have a Standard Score $\mathrm{M}=100, \mathrm{SD}=15$. $* *$ Subtests have an Index Score $\mathrm{M}=10, \mathrm{SD}=3$. 
Table 29

Means, Standard Errors, Standard Deviations, and t-test Results of the Combined WAIS-III/IV Dataset's WJ III GIA and Subtests by Gender

\begin{tabular}{|c|c|c|c|c|c|c|c|c|}
\hline GIA/ Subtest* & Gender & $n$ & M & $\mathrm{SE}$ & $\mathrm{SD}$ & $t$ & df & $p$ \\
\hline GIA & $\begin{array}{l}\text { Female } \\
\text { Male }\end{array}$ & $\begin{array}{l}520 \\
644\end{array}$ & $\begin{array}{l}102.70 \\
103.21\end{array}$ & $\begin{array}{l}0.50 \\
0.50\end{array}$ & $\begin{array}{l}11.48 \\
12.77\end{array}$ & -0.71 & 1162 & .479 \\
\hline $\begin{array}{l}\text { Verbal } \\
\text { Comprehension }\end{array}$ & $\begin{array}{l}\text { Female } \\
\text { Male }\end{array}$ & $\begin{array}{l}474 \\
584\end{array}$ & $\begin{array}{l}102.13 \\
104.32\end{array}$ & $\begin{array}{l}0.55 \\
0.52\end{array}$ & $\begin{array}{l}11.94 \\
12.47\end{array}$ & -2.89 & 1056 & .004 \\
\hline $\begin{array}{l}\text { Visual-Auditory } \\
\text { Learning }\end{array}$ & $\begin{array}{l}\text { Female } \\
\text { Male }\end{array}$ & $\begin{array}{l}522 \\
642\end{array}$ & $\begin{array}{l}99.19 \\
98.29\end{array}$ & $\begin{array}{l}0.83 \\
0.72\end{array}$ & $\begin{array}{l}18.94 \\
18.20\end{array}$ & 0.82 & 1162 & .412 \\
\hline $\begin{array}{l}\text { Spatial } \\
\text { Relations }\end{array}$ & $\begin{array}{l}\text { Female } \\
\text { Male }\end{array}$ & $\begin{array}{l}473 \\
581\end{array}$ & $\begin{array}{l}104.59 \\
105.57\end{array}$ & $\begin{array}{l}0.49 \\
0.48\end{array}$ & $\begin{array}{l}10.67 \\
11.63\end{array}$ & -1.42 & 1052 & .155 \\
\hline $\begin{array}{l}\text { Sound } \\
\text { Blending }\end{array}$ & $\begin{array}{l}\text { Female } \\
\text { Male }\end{array}$ & $\begin{array}{l}520 \\
643\end{array}$ & $\begin{array}{l}105.24 \\
103.24\end{array}$ & $\begin{array}{l}0.53 \\
0.54\end{array}$ & $\begin{array}{l}12.05 \\
13.61\end{array}$ & 2.66 & 1152 & .008 \\
\hline $\begin{array}{l}\text { Concept } \\
\text { Formation }\end{array}$ & $\begin{array}{l}\text { Female } \\
\text { Male }\end{array}$ & $\begin{array}{l}520 \\
645\end{array}$ & $\begin{array}{l}107.34 \\
107.21\end{array}$ & $\begin{array}{l}0.49 \\
0.50\end{array}$ & $\begin{array}{l}11.23 \\
12.78\end{array}$ & 0.18 & 1154 & .860 \\
\hline $\begin{array}{l}\text { Visual } \\
\text { Matching }\end{array}$ & $\begin{array}{l}\text { Female } \\
\text { Male }\end{array}$ & $\begin{array}{l}523 \\
645\end{array}$ & $\begin{array}{l}101.80 \\
100.29\end{array}$ & $\begin{array}{l}0.70 \\
0.61\end{array}$ & $\begin{array}{l}15.90 \\
15.52\end{array}$ & 1.64 & 1166 & .102 \\
\hline $\begin{array}{l}\text { Numbers } \\
\text { Reversed }\end{array}$ & $\begin{array}{l}\text { Female } \\
\text { Male }\end{array}$ & $\begin{array}{l}442 \\
547\end{array}$ & $\begin{array}{l}101.83 \\
104.93\end{array}$ & $\begin{array}{l}0.67 \\
0.65\end{array}$ & $\begin{array}{l}14.09 \\
15.25\end{array}$ & -3.28 & 987 & .001 \\
\hline $\begin{array}{l}\text { Incomplete } \\
\text { Words }\end{array}$ & $\begin{array}{l}\text { Female } \\
\text { Male }\end{array}$ & $\begin{array}{l}517 \\
637\end{array}$ & $\begin{array}{l}106.30 \\
105.55\end{array}$ & $\begin{array}{l}0.60 \\
0.61\end{array}$ & $\begin{array}{l}13.56 \\
15.44\end{array}$ & 0.86 & 1145 & .383 \\
\hline $\begin{array}{l}\text { Retrieval } \\
\text { Fluency }\end{array}$ & $\begin{array}{l}\text { Female } \\
\text { Male }\end{array}$ & $\begin{array}{l}473 \\
582\end{array}$ & $\begin{array}{r}100.07 \\
95.73\end{array}$ & $\begin{array}{l}0.48 \\
0.48\end{array}$ & $\begin{array}{l}10.53 \\
11.46\end{array}$ & 6.34 & 1053 & $<.001$ \\
\hline $\begin{array}{l}\text { Picture } \\
\text { Recognition }\end{array}$ & $\begin{array}{l}\text { Female } \\
\text { Male }\end{array}$ & $\begin{array}{l}518 \\
642\end{array}$ & $\begin{array}{r}103.92 \\
99.65\end{array}$ & $\begin{array}{l}0.51 \\
0.47\end{array}$ & $\begin{array}{l}11.52 \\
11.97\end{array}$ & 6.14 & 1158 & $<.001$ \\
\hline $\begin{array}{l}\text { Analysis- } \\
\text { Synthesis }\end{array}$ & $\begin{array}{l}\text { Female } \\
\text { Male }\end{array}$ & $\begin{array}{l}520 \\
643\end{array}$ & $\begin{array}{l}108.46 \\
110.10\end{array}$ & $\begin{array}{l}0.55 \\
0.57\end{array}$ & $\begin{array}{l}12.51 \\
14.34\end{array}$ & -2.08 & 1154 & .038 \\
\hline $\begin{array}{l}\text { Decision } \\
\text { Speed }\end{array}$ & $\begin{array}{l}\text { Female } \\
\text { Male }\end{array}$ & $\begin{array}{l}474 \\
582\end{array}$ & $\begin{array}{r}103.62 \\
99.88\end{array}$ & $\begin{array}{l}0.72 \\
0.68\end{array}$ & $\begin{array}{l}15.57 \\
16.50\end{array}$ & 3.75 & 1054 & $<.001$ \\
\hline $\begin{array}{l}\text { Memory for } \\
\text { Words }\end{array}$ & $\begin{array}{l}\text { Female } \\
\text { Male }\end{array}$ & $\begin{array}{l}519 \\
643\end{array}$ & $\begin{array}{l}102.97 \\
102.95\end{array}$ & $\begin{array}{l}1.62 \\
0.54\end{array}$ & $\begin{array}{l}36.96 \\
13.69\end{array}$ & 0.01 & 1160 & .992 \\
\hline $\begin{array}{l}\text { Rapid Picture } \\
\text { Naming }\end{array}$ & $\begin{array}{l}\text { Female } \\
\text { Male }\end{array}$ & $\begin{array}{l}470 \\
581\end{array}$ & $\begin{array}{l}98.68 \\
94.35\end{array}$ & $\begin{array}{l}0.71 \\
0.71\end{array}$ & $\begin{array}{l}15.39 \\
17.01\end{array}$ & 4.33 & 1036 & $<.001$ \\
\hline Planning & $\begin{array}{l}\text { Female } \\
\text { Male }\end{array}$ & $\begin{array}{l}470 \\
577\end{array}$ & $\begin{array}{l}110.43 \\
114.57\end{array}$ & $\begin{array}{l}0.99 \\
1.09\end{array}$ & $\begin{array}{l}21.36 \\
26.13\end{array}$ & -2.82 & 1045 & .005 \\
\hline $\begin{array}{l}\text { Pair } \\
\text { Cancellation }\end{array}$ & $\begin{array}{l}\text { Female } \\
\text { Male }\end{array}$ & $\begin{array}{l}474 \\
582\end{array}$ & $\begin{array}{l}99.29 \\
99.50\end{array}$ & $\begin{array}{l}0.67 \\
0.62\end{array}$ & $\begin{array}{l}14.51 \\
15.04\end{array}$ & -0.24 & 1054 & .815 \\
\hline
\end{tabular}


Table 29

Means, Standard Errors, Standard Deviations, and t-test Results of the Combined WAIS-III/IV Dataset's WJ III GIA and Subtests by Gender, continued

Note. WJ III = Woodcock-Johnson Tests of Cognitive Abilities - Third Edition. GIA = General Intellectual Ability. $M=$ Mean. $S E=$ Standard Error of the Mean. SD = Standard Deviation. $\mathrm{df}=$ degrees of freedom. *WJ III Cognitive GIA and subtests have a Standard Score $\mathrm{M}=100, \mathrm{SD}=15$.

Table 30

Means, Standard Errors, Standard Deviations, and t-test Results of the Combined WAIS-III/IV Dataset's WJ III Achievement Subtests by Gender

\begin{tabular}{|c|c|c|c|c|c|c|c|c|}
\hline Subtest* & Gender & $n$ & $\mathrm{M}$ & SE & SD & $t$ & $\mathrm{df}$ & $p$ \\
\hline \multirow{2}{*}{$\begin{array}{l}\text { Letter-Word } \\
\text { Identification }\end{array}$} & Female & 524 & 104.20 & 0.44 & 10.01 & & & \\
\hline & Male & 649 & 103.76 & 0.44 & 11.30 & 0.71 & 1161 & .476 \\
\hline \multirow{2}{*}{$\begin{array}{l}\text { Reading } \\
\text { Fluency }\end{array}$} & Female & 475 & 103.15 & 0.68 & 14.83 & & & \\
\hline & Male & 584 & 99.78 & 0.59 & 14.22 & 3.75 & 1057 & $<.001$ \\
\hline \multirow[t]{2}{*}{ Calculation } & Female & 520 & 108.21 & 0.60 & 13.59 & & & \\
\hline & Male & 644 & 113.18 & 0.65 & 16.47 & -5.64 & 1161 & $<.001$ \\
\hline \multirow[t]{2}{*}{ Math Fluency } & Female & 475 & 99.10 & 0.62 & 13.57 & & & \\
\hline & Male & 583 & 101.42 & 0.59 & 14.34 & -2.69 & 1056 & .007 \\
\hline \multirow[t]{2}{*}{ Spelling } & Female & 473 & 107.34 & 0.44 & 9.60 & & & \\
\hline & Male & 587 & 106.01 & 0.45 & 10.87 & 2.12 & 1049 & .035 \\
\hline \multirow{2}{*}{$\begin{array}{l}\text { Writing } \\
\text { Fluency }\end{array}$} & Female & 522 & 111.00 & 0.61 & 14.02 & & & \\
\hline & Male & 635 & 104.42 & 0.56 & 14.03 & 7.95 & 1155 & $<.001$ \\
\hline \multirow{2}{*}{$\begin{array}{l}\text { Passage } \\
\text { Comprehension }\end{array}$} & Female & 523 & 104.21 & 0.45 & 10.36 & & & \\
\hline & Male & 648 & 105.24 & 0.44 & 11.13 & -1.63 & 1169 & .104 \\
\hline \multirow{2}{*}{$\begin{array}{l}\text { Applied } \\
\text { Problems }\end{array}$} & Female & 526 & 103.39 & 0.49 & 11.29 & & & \\
\hline & Male & 642 & 110.02 & 0.55 & 13.86 & -8.90 & 1166 & $<.001$ \\
\hline \multirow{2}{*}{$\begin{array}{l}\text { Writing } \\
\text { Samples }\end{array}$} & Female & 525 & 109.45 & 0.67 & 15.31 & & & \\
\hline & Male & 647 & 107.93 & 0.66 & 16.70 & 1.61 & 1170 & .109 \\
\hline \multirow[t]{2}{*}{ Word Attack } & Female & 519 & 101.59 & 0.49 & 11.17 & & & \\
\hline & Male & 626 & 102.28 & 0.45 & 11.27 & -1.04 & 1143 & .297 \\
\hline
\end{tabular}

Note. WJ III Achievement = Woodcock-Johnson Tests of Achievement - Third Edition. WAIS-III/IV = Wechsler Adult Intelligence Scale - Third/Fourth Editions. $M=$ Mean. $\mathrm{SE}=$ Standard Error of the Mean. $\mathrm{SD}=$ Standard Deviation. $\mathrm{df}=$ degrees of freedom. *WJ III Achievement subtests have a Standard Score M $=100, \mathrm{SD}=15$. 
Regarding the Conners Adult ADHD Rating Scale with which clinicians can assess self- and other-reported symptoms of ADHD, men exhibited significantly higher mean scores than women only on the self-rated DSM-IV scales of Inattentive Symptoms, Hyperactive/Impulsive Symptoms, and total ADHD Symptoms. As illustrated in Table 31 , women scored significantly higher in every other category except self-reported Problems with Self Concept and other-reported DSM-IV Inattentive Symptoms, in which there was no evidence that men's and women's scores were significantly different.

Table 31

Means, Standard Errors, Standard Deviations, and t-test Results of the CAARS Selfand Other-rated Scales of the Combined WAIS-III/IV Dataset by Gender

\begin{tabular}{llccccccc}
\hline Scale* & Gender & $n$ & $\mathrm{M}$ & $\mathrm{SE}$ & $\mathrm{SD}$ & $t$ & $\mathrm{df}$ & $p$ \\
\hline S-A - Inattention/ & Female & 532 & 66.07 & 0.60 & 13.84 & & & \\
Memory Problems & Male & 651 & 62.00 & 0.51 & 12.97 & 5.17 & 1103 & $<.001$ \\
S-B - Hyperactivity/ & Female & 532 & 57.40 & 0.53 & 12.22 & & & \\
Restlessness & Male & 651 & 53.37 & 0.46 & 11.85 & 5.74 & 1181 & $<.001$ \\
S-C - Impulsivity/ & Female & 532 & 55.86 & 0.57 & 13.17 & & & \\
Emotional Lability & Male & 651 & 51.08 & 0.46 & 11.74 & 6.52 & 1075 & $<.001$ \\
S-D - Problems with & Female & 532 & 55.80 & 0.53 & 12.22 & & & \\
Self Concept & Male & 651 & 54.79 & 0.46 & 11.75 & 1.45 & 1181 & .148 \\
S-E - DSM-IV & Female & 532 & 70.33 & 0.63 & 14.52 & & & \\
Inattentive Symptoms & Male & 651 & 74.88 & 0.61 & 15.44 & -5.21 & 1158 & $<.001$ \\
S-F - DSM-IV Hyperactive- & Female & 532 & 56.51 & 0.64 & 14.81 & & & \\
Impulsive Symptoms & Male & 650 & 58.79 & 0.62 & 15.89 & -2.53 & 1180 & .012 \\
S-G- DSM-IV & Female & 532 & 65.90 & 0.64 & 14.82 & & & \\
ADHD Symptoms & Male & 650 & 70.74 & 0.63 & 16.06 & -5.38 & 1163 & $<.001$ \\
S-H - ADHD & Female & 532 & 61.51 & 0.49 & 11.25 & & & \\
Index & Male & 650 & 57.68 & 0.45 & 11.51 & 5.74 & 1180 & $<.001$ \\
O-A - Inattention/ & Female & 328 & 63.32 & 0.76 & 13.75 & & & \\
Memory Problems & Male & 401 & 60.17 & 0.70 & 14.00 & 3.05 & 727 & .002 \\
O-B - Hyperactivity/ & Female & 328 & 56.46 & 0.72 & 12.97 & & & \\
Restlessness & Male & 401 & 53.13 & 0.64 & 12.88 & 3.46 & 727 & .001 \\
O-C - Impulsivity/ & Female & 328 & 54.79 & 0.63 & 11.37 & & & \\
Emotional Lability & Male & 401 & 50.29 & 0.52 & 10.38 & 5.59 & 727 & $<.001$
\end{tabular}


Table 31

Means, Standard Errors, Standard Deviations, and t-test Results of the CAARS Self-and Other-rated Scales of the Combined WAIS-III/IV Dataset by Gender, continued

\begin{tabular}{llccccccc}
\hline Scale* & Gender & $n$ & $\mathrm{M}$ & $\mathrm{SE}$ & $\mathrm{SD}$ & $t$ & df & $p$ \\
\hline O-D - Problems with & Female & 328 & 56.34 & 0.66 & 11.99 & & & \\
Self Concept & Male & 401 & 53.51 & 0.58 & 11.63 & 3.23 & 727 & .001 \\
O- E - DSM-IV & Female & 328 & 61.86 & 0.67 & 12.10 & & & \\
Inattentive Symptoms & Male & 401 & 60.62 & 0.65 & 13.07 & 1.31 & 727 & .189 \\
O-F - DSM-IV Hyperactive- & Female & 329 & 56.90 & 0.74 & 13.49 & & & \\
Impulsive Symptoms & Male & 401 & 53.22 & 0.63 & 12.56 & 3.81 & 728 & $<.001$ \\
O-G - DSM-IV & Female & 328 & 61.45 & 0.73 & 13.30 & & & \\
ADHD Symptoms & Male & 401 & 57.86 & 0.61 & 12.25 & 3.79 & 727 & $<.001$ \\
O-H ADHD & Female & 328 & 61.09 & 0.68 & 12.23 & & & \\
Index & Male & 401 & 56.30 & 0.56 & 11.15 & 5.52 & 727 & $<.001$ \\
\hline
\end{tabular}

Note. CAARS $=$ Conners Adult ADHD Rating Scale. WAIS-III/IV = Wechsler Adult Intelligence Scale - Third/Fourth Editions. $\mathrm{M}=$ Mean. $\mathrm{SE}=$ Standard Error of the Mean. $\mathrm{SD}=$ Standard Deviation. $\mathrm{df}=$ degrees of freedom. $\mathrm{S}=$ Self-Rated Scale. $\mathrm{O}=$ Other Rated Scale. ${ }^{*}$ CAARS Rating Scales have a T-score $\mathrm{M}=50, \mathrm{SD}=10$.

As shown in Table 32, there was no evidence of significant gender differences in the SCL-90-R scores and the BDI-II total score means only trended toward significance, with the women scoring slightly higher than the men.

Table 32

Means, Standard Errors, Standard Deviations, and t-test Results of the Combined WAISIII/IV Dataset's BDI-II Total Score and SCL-90-R GSI by Gender

\begin{tabular}{llcccrcrc}
\hline Scale* & Gender & $n$ & M & SE & SD & $t$ & df & $p$ \\
\hline BDI-II Total Score & Female & 482 & 14.33 & 0.49 & 10.70 & & & \\
& Male & 596 & 13.10 & 0.41 & 9.98 & 1.91 & 997 & .055 \\
SCL-90-R GSI & Female & 454 & 58.28 & 0.55 & 11.78 & & & \\
& Male & 557 & 58.92 & 0.56 & 13.26 & -0.81 & 1001 & .420 \\
\hline
\end{tabular}

Note. WAIS-III/IV = Wechsler Adult Intelligence Scale - Third/Fourth Editions. BDI-II $=$ Beck Depression Inventory - Second Edition. SCL-90-R GSI = Symptom Checklist90-Revised Global Severity Index. $\mathrm{M}=$ Mean. SE = Standard Error of the Mean. SD = Standard Deviation. $\mathrm{df}=$ degrees of freedom. ${ }^{*}$ Cut score guidelines for the BDI-II Total Score: 0-13 is considered minimal range, 14-19 is mild, 20-28 is moderate, and 2963 is severe for symptoms of depression; the GSI has a T-score $\mathrm{M}=50, \mathrm{SD}=10$. 
Table 33 shows results of a cross-tabulation chi square analysis of diagnosis by gender. After considering gender differences in the number of diagnoses of any disorder (a higher proportion of women than men were diagnosed), gender differences are noted in the proportion of men and women given a particular diagnosis compared with the number of men and women not given that diagnosis. Also, the proportion of students given a primary diagnosis of a particular disorder was compared to the proportion of students given a secondary diagnosis of that disorder. Between men and women, only depression and anxiety produced significant results, with both disorders showing higher proportions of diagnoses for women than for men, although the difference in overall depression only trended toward significance. However, whereas men were diagnosed proportionally higher with primary depression, women received proportionally more secondary diagnoses. Women also received proportionally more diagnoses of anxiety, but no proportional differences between men and women were seen between primary and secondary anxiety diagnoses. 
Table 33

Cross-tabulations of Diagnosis and Gender from the WAIS-III/IV Combined Dataset

\begin{tabular}{|c|c|c|c|c|c|c|c|c|c|c|}
\hline \multicolumn{2}{|c|}{ Diagnosis } & \multicolumn{4}{|c|}{ Gender } & \multirow[t]{3}{*}{ Total } & \multirow[t]{3}{*}{$\%$} & \multirow[t]{3}{*}{$\chi^{2}$} & \multirow[t]{3}{*}{ df } & \multirow[t]{3}{*}{$p$} \\
\hline & & \multicolumn{2}{|c|}{ Female } & \multicolumn{2}{|c|}{$\underline{\text { Male }}$} & & & & & \\
\hline & & $n$ & $\%$ & $n$ & $\%$ & & & & & \\
\hline Overall & No & 51 & 8.7 & 90 & 12.7 & 141 & 10.9 & & & \\
\hline Diagnosis & Yes & 535 & 91.3 & 616 & 87.3 & 1151 & 89.1 & 5.39 & 1 & .020 \\
\hline \multirow[t]{4}{*}{ Depression } & No & 421 & 71.8 & 540 & 76.5 & 961 & 74.4 & & & \\
\hline & Yes & 165 & 28.2 & 166 & 23.5 & 331 & 25.6 & 3.62 & 1 & .057 \\
\hline & Primary & 15 & 2.6 & 34 & 4.8 & 49 & 3.8 & & & \\
\hline & Secondary & 150 & 25.6 & 132 & 18.7 & 282 & 21.8 & 12.21 & 2 & .002 \\
\hline \multirow[t]{4}{*}{ Anxiety } & No & 458 & 78.3 & 584 & 80.7 & 1043 & 80.7 & & & \\
\hline & Yes & 127 & 21.7 & 122 & 17.3 & 249 & 19.3 & 3.97 & 1 & .046 \\
\hline & Primary & 35 & 6.0 & 31 & 4.4 & 66 & 5.1 & & & \\
\hline & Secondary & 93 & 15.9 & 91 & 12.9 & 184 & 14.2 & 4.39 & 2 & .111 \\
\hline Total & & 586 & 100.0 & 706 & 100.0 & 1292 & 100.0 & & & \\
\hline
\end{tabular}

Note. WAIS-III/IV = Wechsler Adult Intelligence Scale - Third/Fourth Editions. Table compares men and women regarding any diagnosis, depression diagnosis (yes or no) and depression as a primary diagnosis versus depression as a secondary diagnosis. $\%=$ percentage of students in the database. $\chi^{2}=$ chi square. $\mathrm{df}=$ degrees of freedom.

\section{Ethnicity}

Tables 34 - 38 illustrate score differences by ethnicity for the WAIS-III, WJ III, CAARS, BDI-II, and SCL-90-R. All cognitive subtests exhibited significant ethnic group differences except Cancellation on the WAIS-IV (for which there may have been too few students to provide a valid analysis), and Visual Matching and Memory for Words on the WJ-III COG. All WJ III achievement subtests exhibited significant main ethnic group differences. On the CAARS other-rated scale, only the Hyperactivity/ Restlessness and DSM-IV Hyperactive/Impulsive Symptoms scales did not show significant ethnic group differences. All other CAARS scales, the BDI-II total score, and the SCL-90-R GSI exhibited significant group differences. 
As indicated in Tables $34-36$, Scheffé's post hoc analyses revealed that the African American group obtained significantly lower scores than all other ethnic groups on both the WAIS-III/IV FSIQ and WJ III COG GIA. Reflecting the lower scores, African Americans as a group performed more poorly than the other groups on the comprehension-knowledge subtests of Information and Verbal Comprehension; the fluid reasoning subtests of Matrix Reasoning, Concept Formation, and Analysis Synthesis; the visual-spatial processing subtests of Block Design, Visual Puzzles, and Spatial Relations; the working-memory capacity subtest Letter-Number Sequencing; the processing speed subtest Symbol Search, and the auditory processing subtest Sound Blending. The African American group also performed more poorly than other groups on all achievement subtests except Reading Fluency, on which their scores shared the low group with the Latino group's scores. In several subtests, the scores from Asians/Indians, Caucasians, and Latinos formed a single high group and scores from African Americans formed the low group: Information (comprehension-knowledge), Matrix Reasoning and AnalysisSynthesis (fluid reasoning), Letter-Number Sequencing (working-memory capacity), Visual Puzzles and Spatial Relations (visual-spatial processing), Symbol Search (processing speed), and the achievement subtests Spelling, Writing Fluency, and Word Attack (reading and writing knowledge).

There were no subtests in which the African American group performed singularly better than any other group. As a group, African Americans performed as well as or better than other groups only on the fluency measures of Retrieval Fluency (longterm storage and retrieval) and Rapid Picture Naming (processing speed), in which their 
scores shared the high group with those of Caucasians; and on Picture Recognition (visual-spatial processing), in which their scores shared the high group with those of Caucasians and Latinos.

Also noteworthy in Tables $34-36$ is that the Caucasian group performed in the highest group on almost all subtests. The few exceptions include the three subtests in which there were no significant group differences: Visual Matching (visual-spatial processing), Memory for Words (working-memory capacity), and Cancellation (processing speed); and the achievement subtests of Calculation, Math Fluency, and Applied Problems (all quantitative knowledge), in which the Asian/Indian group performed best. The Caucasian group performed better than all other groups on the comprehension-knowledge subtests Vocabulary, Similarities, and Verbal Comprehension, the auditory processing subtest Sound Blending, the processing speed subtest Coding; and on the achievement reading and writing knowledge subtest Writing Samples. The Caucasian and Asian/Indian groups' scores shared the high group on the WAIS-III/IV FSIQ and WJ III GIA (in which Asian/Indian group scores, although in the high group with the Caucasian group's scores, were also similar to Latino group scores), the comprehension-knowledge subtest Comprehension, the fluid reasoning subtest Concept Formation, the visual-spatial processing subtest Block Design, the workingmemory capacity subtests Digit Span and Numbers Reversed, the quantitative knowledge/fluid reasoning/working memory capacity subtest Arithmetic, and the reading and writing knowledge achievement subtests Letter-Word Identification, Reading Fluency, and Passage Comprehension. 
For many cognitive and achievement subtests, Latinos found themselves in a middle group, often scoring higher than or equal to African Americans or lower than or equal to Caucasians. The Latino group's scores comprised the middle of three groups in both the FSIQ and GIA and were similar to the Asian/Indian group's scores. Although there were no subtests in which Latinos found themselves singularly in the high group, their scores often shared the high group with the scores of other ethnic groups. Latinos' scores shared the high group with those of the Caucasian and Asian/Indian groups on the comprehension-knowledge subtest Information; fluid reasoning subtests Matrix Reasoning and Figure Weights; visual-spatial processing subtests Picture Arrangement, Visual Puzzles, and Spatial Relations; working-memory capacity subtest Letter-Number Sequencing; and processing speed subtest Symbol Search. The Latino group's scores also shared the high group with the Caucasian group's scores on the comprehensionknowledge/visual-spatial processing subtest Picture Completion.

Although the Latino group also found itself in the high group on the visual-spatial processing subtests Picture Recognition and Planning, auditory processing subtest Incomplete Words, long-term storage and retrieval subtest Visual-Auditory Learning, and processing speed subtest Pair Cancellation, its scores in these subtests were not significantly different from the low group's scores. Other Latino group scores that were similar to both the low and high groups include scores from the fluid reasoning subtest Figure Weights, the visual-spatial processing subtest Picture Recognition, the auditory processing subtest Incomplete Words, the working-memory capacity subtests Digit Span and Numbers Reversed, the processing speed subtest Rapid Picture Naming; and the 
achievement reading and writing knowledge subtest Reading Fluency (shared with African Americans' scores), and the quantitative knowledge subtest Math Fluency (shared with African Americans' and Caucasians' scores). Latinos shared the middle of three groups in the comprehension-knowledge subtests Vocabulary, Similarities, Comprehension, and Verbal Comprehension; the fluid reasoning subtest Concept Formation, the visual-spatial processing subtests Block Design and Picture Completion; the auditory processing subtest Sound Blending, the working-memory capacity subtests Digit Span and Numbers Reversed, the achievement reading and writing knowledge subtests Letter-Word Identification, Passage Comprehension, and Writing Samples; and the quantitative knowledge subtests Arithmetic and Calculation.

The Latino group's scores were never singularly in the lowest group but shared the low group with the African American group (and with dissimilar scores from the high group) in the processing speed subtests Coding, Retrieval Fluency, and Rapid Picture Naming; and in the achievement subtests Reading Fluency and Math Fluency. The scores of the WJ III subtest Applied Problems separated into four distinct groups, of which the Latino group fell second lowest. 
Table 34

WAIS-III/IV Combined Dataset ANOVA Results for WAIS-III/IV FSIQ and Subtests by Ethnicity

\begin{tabular}{|c|c|c|c|c|c|c|}
\hline $\begin{array}{l}\text { FSIQ*/ } \\
\text { Subtest** }\end{array}$ & Ethnicity & $n$ & M & SE & $\mathrm{SD}$ & $\begin{array}{c}\text { Group } \\
\text { Differences }\end{array}$ \\
\hline FSIQ & $\begin{array}{l}\text { African American } \\
\text { Asian/Indian } \\
\text { Caucasian } \\
\text { Latino } \\
\text { Total }\end{array}$ & $\begin{array}{r}174 \\
171 \\
703 \\
129 \\
1177\end{array}$ & $\begin{array}{l}101.64^{\mathrm{a}} \\
113.50^{\mathrm{b}, \mathrm{c}} \\
115.62^{\mathrm{c}} \\
110.34^{\mathrm{b}} \\
112.67\end{array}$ & $\begin{array}{l}0.99 \\
1.00 \\
0.48 \\
1.05 \\
0.40\end{array}$ & $\begin{array}{l}13.02 \\
13.03 \\
12.72 \\
11.91 \\
13.61\end{array}$ & $\begin{array}{c}F(3,1173)= \\
57.92, p<.001\end{array}$ \\
\hline Vocabulary & $\begin{array}{l}\text { African American } \\
\text { Asian/Indian } \\
\text { Caucasian } \\
\text { Latino } \\
\text { Total }\end{array}$ & $\begin{array}{r}174 \\
171 \\
704 \\
129 \\
1178\end{array}$ & $\begin{array}{l}10.90^{\mathrm{a}} \\
12.77^{\mathrm{b}} \\
13.58^{\mathrm{c}} \\
12.47^{\mathrm{b}} \\
12.95\end{array}$ & $\begin{array}{l}0.24 \\
0.25 \\
0.11 \\
0.26 \\
0.09\end{array}$ & $\begin{array}{l}3.23 \\
3.22 \\
2.83 \\
2.93 \\
3.10\end{array}$ & $\begin{array}{c}F(3,1174)= \\
39.71, p<.001\end{array}$ \\
\hline Similarities & $\begin{array}{l}\text { African American } \\
\text { Asian/Indian } \\
\text { Caucasian } \\
\text { Latino } \\
\text { Total }\end{array}$ & $\begin{array}{r}174 \\
171 \\
704 \\
129 \\
1178\end{array}$ & $\begin{array}{l}10.34^{\mathrm{a}} \\
12.15^{\mathrm{b}} \\
12.55^{\mathrm{c}} \\
11.71^{\mathrm{b}} \\
12.07\end{array}$ & $\begin{array}{l}0.24 \\
0.24 \\
0.11 \\
0.27 \\
0.09\end{array}$ & $\begin{array}{l}3.23 \\
3.13 \\
2.89 \\
3.07 \\
3.03\end{array}$ & $\begin{array}{c}F(3,1174)= \\
27.10, p<.001\end{array}$ \\
\hline Arithmetic & $\begin{array}{l}\text { African American } \\
\text { Asian/Indian } \\
\text { Caucasian } \\
\text { Latino } \\
\text { Total }\end{array}$ & $\begin{array}{r}174 \\
171 \\
704 \\
129 \\
1178\end{array}$ & $\begin{array}{l}10.12^{\mathrm{a}} \\
12.78^{\mathrm{c}} \\
12.25^{\mathrm{b}, \mathrm{c}} \\
11.56^{\mathrm{b}} \\
11.94\end{array}$ & $\begin{array}{l}0.23 \\
0.18 \\
0.10 \\
0.23 \\
0.08\end{array}$ & $\begin{array}{l}2.97 \\
2.33 \\
2.58 \\
2.58 \\
2.73\end{array}$ & $\begin{array}{c}F(3,1174)= \\
38.43, p<.001\end{array}$ \\
\hline Digit Span & $\begin{array}{l}\text { African American } \\
\text { Asian/Indian } \\
\text { Caucasian } \\
\text { Latino } \\
\text { Total }\end{array}$ & $\begin{array}{r}174 \\
171 \\
704 \\
129 \\
1178\end{array}$ & $\begin{array}{l}10.09^{\mathrm{a}} \\
11.48^{\mathrm{c}} \\
11.13^{\mathrm{b}, \mathrm{c}} \\
10.61^{\mathrm{a}, \mathrm{b}} \\
10.97\end{array}$ & $\begin{array}{l}0.20 \\
0.23 \\
0.11 \\
0.24 \\
0.09\end{array}$ & $\begin{array}{l}2.69 \\
3.05 \\
2.94 \\
2.72 \\
2.93\end{array}$ & $\begin{array}{c}F(3,1174)= \\
8.49, \quad p<.001\end{array}$ \\
\hline Information & $\begin{array}{l}\text { African American } \\
\text { Asian/Indian } \\
\text { Caucasian } \\
\text { Latino } \\
\text { Total }\end{array}$ & $\begin{array}{r}174 \\
171 \\
704 \\
129 \\
1178\end{array}$ & $\begin{array}{l}10.82^{\mathrm{a}} \\
12.88^{\mathrm{b}} \\
13.14^{\mathrm{b}} \\
12.50^{\mathrm{b}} \\
12.69\end{array}$ & $\begin{array}{l}0.23 \\
0.20 \\
0.10 \\
0.22 \\
0.08\end{array}$ & $\begin{array}{l}3.06 \\
2.67 \\
2.55 \\
2.53 \\
2.76\end{array}$ & $\begin{array}{c}F(3,1174)= \\
36.09, p<.001\end{array}$ \\
\hline Comprehension & $\begin{array}{l}\text { African American } \\
\text { Asian/Indian } \\
\text { Caucasian } \\
\text { Latino } \\
\text { Total }\end{array}$ & $\begin{array}{r}173 \\
171 \\
702 \\
129 \\
1175\end{array}$ & $\begin{array}{l}11.12^{\mathrm{a}} \\
12.41^{\mathrm{b}, \mathrm{c}} \\
13.19^{\mathrm{c}} \\
11.97^{\mathrm{b}} \\
12.64\end{array}$ & $\begin{array}{l}0.24 \\
0.22 \\
0.10 \\
0.24 \\
0.08\end{array}$ & $\begin{array}{l}3.18 \\
2.94 \\
2.68 \\
2.70 \\
2.90\end{array}$ & $\begin{array}{c}F(3,1171)= \\
28.90, p<.001\end{array}$ \\
\hline
\end{tabular}


Table 34

WAIS-III/IV Combined Dataset ANOVA Results for WAIS-III/IV FSIQ and Subtests by Ethnicity, continued

\begin{tabular}{|c|c|c|c|c|c|c|}
\hline $\begin{array}{l}\text { FSIQ*/ } \\
\text { Subtest** }\end{array}$ & Ethnicity & $n$ & $\mathrm{M}$ & SE & SD & $\begin{array}{c}\text { Group } \\
\text { Differences }\end{array}$ \\
\hline $\begin{array}{l}\text { Letter- } \\
\text { Number } \\
\text { Sequencing }\end{array}$ & $\begin{array}{l}\text { African American } \\
\text { Asian/Indian } \\
\text { Caucasian } \\
\text { Latino } \\
\text { Total }\end{array}$ & $\begin{array}{r}174 \\
171 \\
704 \\
129 \\
1178\end{array}$ & $\begin{array}{l}9.87^{\mathrm{a}} \\
11.53^{\mathrm{b}} \\
11.59^{\mathrm{b}} \\
10.91^{\mathrm{b}} \\
11.25\end{array}$ & $\begin{array}{l}0.21 \\
0.25 \\
0.12 \\
0.24 \\
0.09\end{array}$ & $\begin{array}{l}2.82 \\
3.29 \\
3.14 \\
2.76 \\
3.13\end{array}$ & $\begin{array}{c}F(3,1174)= \\
15.60, p<.001\end{array}$ \\
\hline $\begin{array}{l}\text { Picture } \\
\text { Completion }\end{array}$ & $\begin{array}{l}\text { African American } \\
\text { Asian/Indian } \\
\text { Caucasian } \\
\text { Latino } \\
\text { Total }\end{array}$ & $\begin{array}{r}174 \\
170 \\
703 \\
129 \\
1176\end{array}$ & $\begin{array}{c}8.94^{\mathrm{a}} \\
9.59^{\mathrm{a}, \mathrm{b}} \\
10.65^{\mathrm{c}} \\
9.88^{\mathrm{b}, \mathrm{c}} \\
10.16\end{array}$ & $\begin{array}{l}0.23 \\
0.23 \\
0.11 \\
0.26 \\
0.09\end{array}$ & $\begin{array}{l}3.07 \\
3.03 \\
2.95 \\
2.95 \\
3.05\end{array}$ & $\begin{array}{c}F(3,1172)= \\
18.49, p<.001\end{array}$ \\
\hline Coding & $\begin{array}{l}\text { African American } \\
\text { Asian/Indian } \\
\text { Caucasian } \\
\text { Latino } \\
\text { Total }\end{array}$ & $\begin{array}{r}174 \\
171 \\
704 \\
129 \\
1178\end{array}$ & & & $\begin{array}{l}2.68 \\
3.15 \\
2.91 \\
2.54 \\
2.90\end{array}$ & $\begin{array}{c}F(3,1174)= \\
6.05, p<.001\end{array}$ \\
\hline Block Design & $\begin{array}{l}\text { African American } \\
\text { Asian/Indian } \\
\text { Caucasian } \\
\text { Latino } \\
\text { Total }\end{array}$ & $\begin{array}{r}174 \\
171 \\
704 \\
129 \\
1178\end{array}$ & $\begin{array}{l}8.94^{\mathrm{a}} \\
11.92^{\mathrm{b}, \mathrm{c}} \\
12.52^{\mathrm{c}} \\
11.63^{\mathrm{b}} \\
11.80\end{array}$ & $\begin{array}{l}0.21 \\
0.22 \\
0.12 \\
0.23 \\
0.09\end{array}$ & $\begin{array}{l}2.72 \\
2.84 \\
3.11 \\
2.66 \\
3.21\end{array}$ & $\begin{array}{c}F(3,1174)= \\
67.49, p<.001\end{array}$ \\
\hline $\begin{array}{l}\text { Matrix } \\
\text { Reasoning }\end{array}$ & $\begin{array}{l}\text { African American } \\
\text { Asian/Indian } \\
\text { Caucasian } \\
\text { Latino } \\
\text { Total }\end{array}$ & $\begin{array}{r}174 \\
171 \\
703 \\
129 \\
1177\end{array}$ & $\begin{array}{l}11.39^{\mathrm{a}} \\
12.78^{\mathrm{b}} \\
13.19^{\mathrm{b}} \\
12.74^{\mathrm{b}} \\
12.82\end{array}$ & & $\begin{array}{l}2.82 \\
2.67 \\
2.44 \\
2.64 \\
2.63\end{array}$ & $\begin{array}{c}F(3,1173)= \\
23.38, p<.001\end{array}$ \\
\hline $\begin{array}{l}\text { Picture } \\
\text { Arrangement }\end{array}$ & $\begin{array}{l}\text { African American } \\
\text { Asian/Indian } \\
\text { Caucasian } \\
\text { Latino } \\
\text { Total }\end{array}$ & $\begin{array}{l}139 \\
116 \\
571 \\
102 \\
928\end{array}$ & $\begin{array}{l}10.55^{\mathrm{a}} \\
11.05^{\mathrm{a}, \mathrm{b}} \\
11.62^{\mathrm{b}} \\
11.49^{\mathrm{b}} \\
11.38\end{array}$ & $\begin{array}{l}0.25 \\
0.28 \\
0.12 \\
0.26 \\
0.09\end{array}$ & $\begin{array}{l}2.90 \\
2.97 \\
2.82 \\
2.65 \\
2.86\end{array}$ & $\begin{array}{c}F(3,924)= \\
5.86, p=.001\end{array}$ \\
\hline Visual Puzzles & $\begin{array}{l}\text { African American } \\
\text { Asian/Indian } \\
\text { Caucasian } \\
\text { Latino } \\
\text { Total }\end{array}$ & $\begin{array}{r}35 \\
54 \\
131 \\
27 \\
247\end{array}$ & $\begin{array}{r}8.80^{\mathrm{a}} \\
11.41^{\mathrm{b}} \\
11.36^{\mathrm{b}} \\
11.48^{\mathrm{b}} \\
11.02\end{array}$ & $\begin{array}{l}0.37 \\
0.36 \\
0.25 \\
0.54 \\
0.18\end{array}$ & $\begin{array}{l}2.17 \\
2.64 \\
2.91 \\
2.82 \\
2.88\end{array}$ & $\begin{array}{c}F(3,243)= \\
8.88, p<.001\end{array}$ \\
\hline
\end{tabular}


Table 34

WAIS-III/IV Combined Dataset ANOVA Results for WAIS-III/IV FSIQ and Subtests by Ethnicity, continued

\begin{tabular}{|c|c|c|c|c|c|c|}
\hline $\begin{array}{l}\text { FSIQ*/ } \\
\text { Subtest** }\end{array}$ & Ethnicity & $n$ & M & $\mathrm{SE}$ & SD & $\begin{array}{c}\text { Group } \\
\text { Differences }\end{array}$ \\
\hline \multirow[t]{5}{*}{ Figure Weights } & African American & 35 & $10.66^{\mathrm{a}}$ & 0.53 & 3.16 & \\
\hline & Asian/Indian & 54 & $13.39^{b}$ & 0.38 & 2.80 & \\
\hline & Caucasian & 130 & $12.66^{\mathrm{b}}$ & 0.24 & 2.69 & \\
\hline & Latino & 27 & $12.04^{\mathrm{a}, \mathrm{b}}$ & 0.58 & 2.99 & $F(3,242)=$ \\
\hline & Total & 246 & 12.47 & 0.19 & 2.92 & $7.15, p<.001$ \\
\hline \multirow[t]{5}{*}{ Symbol Search } & African American & 174 & $9.83^{\mathrm{a}}$ & 0.20 & 2.62 & \\
\hline & Asian/Indian & 171 & $11.12^{b}$ & 0.26 & 3.45 & \\
\hline & Caucasian & 703 & $11.22^{\mathrm{b}}$ & 0.11 & 2.99 & \\
\hline & Latino & 129 & $10.78^{b}$ & 0.23 & 2.62 & $F(3,1173)=$ \\
\hline & Total & 1177 & 10.95 & 0.09 & 3.01 & $10.60, p<.001$ \\
\hline \multirow[t]{5}{*}{ Cancellation } & African American & 9 & $9.33^{\mathrm{a}}$ & 1.17 & 3.50 & \\
\hline & Asian/Indian & 14 & $9.57^{\mathrm{a}}$ & 0.86 & 3.20 & \\
\hline & Caucasian & 35 & $9.69^{\mathrm{a}}$ & 0.45 & 2.64 & \\
\hline & Latino & 9 & $9.67^{\mathrm{a}}$ & 1.43 & 4.30 & $F(3,63)=$ \\
\hline & Total & 67 & 9.61 & 0.37 & 3.06 & $0.03, p=.992$ \\
\hline
\end{tabular}

Note. WAIS-III/IV = Wechsler Adult Intelligence Scale - Third/Fourth Editions. FSIQ $=$ Full-Scale Intelligence Quotient. $\mathrm{M}=$ Mean. $\mathrm{SE}=$ Standard Error of the Mean. SD = Standard Deviation. *WAIS-III/IV FSIQs have a Standard Score M = 100, SD = 15. $* *$ Subtests have an Index Score $\mathrm{M}=10, \mathrm{SD}=3$. Means sharing the same superscript are not significantly different from each other (Scheffé's, $p<0.05$ ). 
Table 35

WAIS-III/IV Combined Dataset ANOVA Results for the WJ III Cognitive GIA and Subtests by Ethnicity

\begin{tabular}{|c|c|c|c|c|c|c|}
\hline GIA/ Subtest* & Ethnicity & $n$ & M & $\mathrm{SE}$ & SD & Group Differences \\
\hline GIA & $\begin{array}{l}\text { African American } \\
\text { Asian/Indian } \\
\text { Caucasian } \\
\text { Latino } \\
\text { Total }\end{array}$ & $\begin{array}{r}172 \\
166 \\
696 \\
125 \\
1159\end{array}$ & $\begin{array}{l}94.37^{\mathrm{a}} \\
102.59^{\mathrm{b}, \mathrm{c}} \\
105.60^{\mathrm{c}} \\
100.86^{\mathrm{b}} \\
102.99\end{array}$ & $\begin{array}{l}0.93 \\
0.91 \\
0.43 \\
1.06 \\
0.36\end{array}$ & $\begin{array}{l}12.20 \\
11.73 \\
11.30 \\
11.85 \\
12.20\end{array}$ & $\begin{array}{c}F(3,1155)= \\
45.25, p<.001\end{array}$ \\
\hline $\begin{array}{l}\text { Verbal } \\
\text { Comprehension }\end{array}$ & $\begin{array}{l}\text { African American } \\
\text { Asian/Indian } \\
\text { Caucasian } \\
\text { Latino } \\
\text { Total }\end{array}$ & $\begin{array}{r}156 \\
154 \\
624 \\
119 \\
1053\end{array}$ & $\begin{array}{l}93.80^{\mathrm{a}} \\
101.95^{\mathrm{b}} \\
106.61^{\mathrm{c}} \\
100.47^{\mathrm{b}} \\
103.33\end{array}$ & $\begin{array}{l}1.01 \\
0.93 \\
0.44 \\
1.10 \\
0.38\end{array}$ & $\begin{array}{l}12.63 \\
11.58 \\
10.95 \\
11.98 \\
12.29\end{array}$ & $\begin{array}{c}F(3,1049)= \\
56.55, p<.001\end{array}$ \\
\hline $\begin{array}{l}\text { Visual- } \\
\text { Auditory } \\
\text { Learning }\end{array}$ & $\begin{array}{l}\text { African American } \\
\text { Asian/Indian } \\
\text { Caucasian } \\
\text { Latino } \\
\text { Total }\end{array}$ & $\begin{array}{r}171 \\
166 \\
696 \\
126 \\
1159\end{array}$ & $\begin{array}{c}92.31^{\mathrm{a}} \\
98.19^{\mathrm{b}} \\
100.77^{\mathrm{b}} \\
97.25^{\mathrm{a}, \mathrm{b}} \\
98.77\end{array}$ & $\begin{array}{l}1.36 \\
1.49 \\
0.65 \\
1.93 \\
0.54\end{array}$ & $\begin{array}{l}17.85 \\
19.21 \\
17.23 \\
21.71 \\
18.36\end{array}$ & $\begin{array}{c}F(3,1155)= \\
10.41, p<.001\end{array}$ \\
\hline $\begin{array}{l}\text { Spatial } \\
\text { Relations }\end{array}$ & $\begin{array}{l}\text { African American } \\
\text { Asian/Indian } \\
\text { Caucasian } \\
\text { Latino } \\
\text { Total }\end{array}$ & $\begin{array}{r}156 \\
153 \\
621 \\
119 \\
1049\end{array}$ & $\begin{array}{l}98.13^{\mathrm{a}} \\
106.27^{\mathrm{b}} \\
106.62^{\mathrm{b}} \\
104.94^{\mathrm{b}} \\
105.12\end{array}$ & $\begin{array}{l}0.89 \\
0.83 \\
0.45 \\
0.80 \\
0.35\end{array}$ & $\begin{array}{r}11.06 \\
10.25 \\
11.29 \\
8.76 \\
11.23\end{array}$ & $\begin{array}{c}F(3,1045)= \\
26.13, p<.001\end{array}$ \\
\hline $\begin{array}{l}\text { Sound } \\
\text { Blending }\end{array}$ & $\begin{array}{l}\text { African American } \\
\text { Asian/Indian } \\
\text { Caucasian } \\
\text { Latino } \\
\text { Total }\end{array}$ & $\begin{array}{r}171 \\
166 \\
696 \\
125 \\
1158\end{array}$ & $\begin{array}{l}95.60^{\mathrm{a}} \\
100.87^{\mathrm{b}} \\
107.22^{\mathrm{c}} \\
103.18^{\mathrm{b}} \\
104.16\end{array}$ & $\begin{array}{l}0.92 \\
1.04 \\
0.44 \\
1.23 \\
0.38\end{array}$ & $\begin{array}{l}12.06 \\
13.46 \\
11.70 \\
13.78 \\
12.96\end{array}$ & $\begin{array}{c}F(3,1154)= \\
46.46, p<.001\end{array}$ \\
\hline $\begin{array}{l}\text { Concept } \\
\text { Formation }\end{array}$ & $\begin{array}{l}\text { African American } \\
\text { Asian/Indian } \\
\text { Caucasian } \\
\text { Latino } \\
\text { Total }\end{array}$ & $\begin{array}{r}171 \\
167 \\
697 \\
125 \\
1160\end{array}$ & $\begin{array}{l}100.57^{\mathrm{a}} \\
107.20^{\mathrm{b}, \mathrm{c}} \\
109.48^{\mathrm{c}} \\
104.28^{\mathrm{b}} \\
107.28\end{array}$ & $\begin{array}{l}0.97 \\
0.86 \\
0.44 \\
1.01 \\
0.36\end{array}$ & $\begin{array}{l}12.64 \\
11.12 \\
11.66 \\
11.34 \\
12.13\end{array}$ & $\begin{array}{c}F(3,1156)= \\
29.65, p<.001\end{array}$ \\
\hline $\begin{array}{l}\text { Visual } \\
\text { Matching }\end{array}$ & $\begin{array}{l}\text { African American } \\
\text { Asian/Indian } \\
\text { Caucasian } \\
\text { Latino } \\
\text { Total }\end{array}$ & $\begin{array}{r}171 \\
168 \\
698 \\
126 \\
1163\end{array}$ & $\begin{array}{r}99.46 \\
102.76 \\
101.26 \\
98.81 \\
100.95\end{array}$ & $\begin{array}{l}1.18 \\
1.33 \\
0.59 \\
1.27 \\
0.46\end{array}$ & $\begin{array}{l}15.45 \\
17.29 \\
15.60 \\
14.29 \\
15.72\end{array}$ & $\begin{array}{c}F(3,1159)= \\
2.13, p<.094\end{array}$ \\
\hline
\end{tabular}


Table 35

WAIS-III/IV Combined Dataset ANOVA Results for the WJ III Cognitive GIA and Subtests by Ethnicity, continued

\begin{tabular}{|c|c|c|c|c|c|c|}
\hline GIA/ Subtest* & Ethnicity & $n$ & M & SE & SD & Group Differences \\
\hline $\begin{array}{l}\text { Numbers } \\
\text { Reversed }\end{array}$ & $\begin{array}{l}\text { African American } \\
\text { Asian/Indian } \\
\text { Caucasian } \\
\text { Latino } \\
\text { Total }\end{array}$ & $\begin{array}{l}147 \\
149 \\
571 \\
117 \\
984\end{array}$ & $\begin{array}{c}99.01^{\mathrm{a}} \\
108.02^{\mathrm{c}} \\
103.81^{\mathrm{b}, \mathrm{c}} \\
102.21^{\mathrm{a}, \mathrm{b}} \\
103.54\end{array}$ & $\begin{array}{l}1.22 \\
1.20 \\
0.61 \\
1.36 \\
0.47\end{array}$ & $\begin{array}{l}14.74 \\
14.63 \\
14.58 \\
14.74 \\
14.82\end{array}$ & $\begin{array}{c}F(3,980)= \\
9.73, p<.001\end{array}$ \\
\hline $\begin{array}{l}\text { Incomplete } \\
\text { Words }\end{array}$ & $\begin{array}{l}\text { African American } \\
\text { Asian/Indian } \\
\text { Caucasian } \\
\text { Latino } \\
\text { Total }\end{array}$ & $\begin{array}{r}170 \\
163 \\
692 \\
124 \\
1149\end{array}$ & $\begin{array}{l}101.87^{\mathrm{a}} \\
101.02^{\mathrm{a}} \\
108.26^{\mathrm{b}} \\
104.49^{\mathrm{a}, \mathrm{b}} \\
105.88\end{array}$ & $\begin{array}{l}1.00 \\
1.20 \\
0.54 \\
1.36 \\
0.43\end{array}$ & $\begin{array}{l}13.03 \\
15.36 \\
14.26 \\
15.13 \\
14.65\end{array}$ & $\begin{array}{c}F(3,1145)= \\
17.38, p<.001\end{array}$ \\
\hline $\begin{array}{l}\text { Retrieval } \\
\text { Fluency }\end{array}$ & $\begin{array}{l}\text { African American } \\
\text { Asian/Indian } \\
\text { Caucasian } \\
\text { Latino } \\
\text { Total }\end{array}$ & $\begin{array}{r}156 \\
154 \\
621 \\
119 \\
1050\end{array}$ & $\begin{array}{l}96.50^{\mathrm{a} \cdot \mathrm{b}} \\
95.22^{\mathrm{a}} \\
99.20^{\mathrm{b}} \\
94.40^{\mathrm{a}} \\
97.67\end{array}$ & $\begin{array}{l}0.90 \\
0.84 \\
0.45 \\
1.09 \\
0.35\end{array}$ & $\begin{array}{l}11.24 \\
10.44 \\
11.10 \\
11.92 \\
11.27\end{array}$ & $\begin{array}{c}F(3,1046)= \\
10.39, p<.001\end{array}$ \\
\hline $\begin{array}{l}\text { Picture } \\
\text { Recognition }\end{array}$ & $\begin{array}{l}\text { African American } \\
\text { Asian/Indian } \\
\text { Caucasian } \\
\text { Latino } \\
\text { Total }\end{array}$ & $\begin{array}{r}170 \\
167 \\
692 \\
126 \\
1155\end{array}$ & $\begin{array}{c}99.51^{\mathrm{a}, \mathrm{b}} \\
98.66^{\mathrm{a}} \\
102.81^{\mathrm{b}} \\
100.99^{\mathrm{a}, \mathrm{b}} \\
101.53\end{array}$ & $\begin{array}{l}0.82 \\
0.91 \\
0.47 \\
0.97 \\
0.35\end{array}$ & $\begin{array}{l}10.68 \\
11.77 \\
12.32 \\
10.88 \\
11.96\end{array}$ & $\begin{array}{c}F(3,1151)= \\
7.68, p<.001\end{array}$ \\
\hline $\begin{array}{l}\text { Analysis- } \\
\text { Synthesis }\end{array}$ & $\begin{array}{l}\text { African American } \\
\text { Asian/Indian } \\
\text { Caucasian } \\
\text { Latino } \\
\text { Total }\end{array}$ & $\begin{array}{r}171 \\
166 \\
695 \\
126 \\
1158\end{array}$ & $\begin{array}{l}101.37^{\mathrm{a}} \\
113.13^{\mathrm{b}} \\
110.44^{\mathrm{b}} \\
109.34^{\mathrm{b}} \\
109.37^{2}\end{array}$ & $\begin{array}{l}1.18 \\
1.03 \\
0.47 \\
1.20 \\
0.40\end{array}$ & $\begin{array}{l}15.49 \\
13.23 \\
12.41 \\
13.49 \\
13.58\end{array}$ & $\begin{array}{c}F(3,1154)= \\
27.16, p<.001\end{array}$ \\
\hline $\begin{array}{l}\text { Decision } \\
\text { Speed }\end{array}$ & $\begin{array}{l}\text { African American } \\
\text { Asian/Indian } \\
\text { Caucasian } \\
\text { Latino } \\
\text { Total }\end{array}$ & $\begin{array}{r}156 \\
154 \\
622 \\
119 \\
1051\end{array}$ & $\begin{array}{c}97.55^{\mathrm{a}} \\
99.67^{\mathrm{a}, \mathrm{b}} \\
103.33^{\mathrm{b}} \\
99.75^{\mathrm{a}, \mathrm{b}} \\
101.53\end{array}$ & $\begin{array}{l}1.27 \\
1.32 \\
0.65 \\
1.34 \\
0.50\end{array}$ & $\begin{array}{l}15.85 \\
16.39 \\
16.30 \\
14.57 \\
16.20\end{array}$ & $\begin{array}{c}F(3,1047)= \\
6.98, p<.001\end{array}$ \\
\hline $\begin{array}{l}\text { Memory for } \\
\text { Words }\end{array}$ & $\begin{array}{l}\text { African American } \\
\text { Asian/Indian } \\
\text { Caucasian } \\
\text { Latino } \\
\text { Total }\end{array}$ & $\begin{array}{r}171 \\
166 \\
695 \\
125 \\
1157\end{array}$ & $\begin{array}{l}96.53^{\mathrm{a}} \\
102.39^{\mathrm{b}} \\
103.89^{\mathrm{b}} \\
100.38^{\mathrm{a}, \mathrm{b}} \\
102.92\end{array}$ & $\begin{array}{l}1.19 \\
0.91 \\
0.52 \\
1.22 \\
0.79\end{array}$ & $\begin{array}{l}15.58 \\
11.69 \\
13.61 \\
13.59 \\
13.89\end{array}$ & $\begin{array}{c}F(3,1153)= \\
14.12, p<.001\end{array}$ \\
\hline
\end{tabular}


Table 35

WAIS-III/IV Combined Dataset ANOVA Results for the WJ III Cognitive GIA and Subtests by Ethnicity, continued

\begin{tabular}{llccccc}
\hline GIA/ Subtest* & \multicolumn{1}{c}{ Ethnicity } & $n$ & $\mathrm{M}$ & $\mathrm{SE}$ & $\mathrm{SD}$ & Group Differences \\
\hline Rapid & African American & 155 & $96.06^{\mathrm{b}}$ & 1.21 & 15.07 & \\
Picture & Asian/Indian & 154 & $91.18^{\mathrm{a}}$ & 1.38 & 17.11 & \\
Naming & Caucasian & 618 & $98.61^{\mathrm{b}}$ & 0.66 & 16.28 & \\
& Latino & 119 & $91.01^{\mathrm{a}}$ & 1.45 & 15.81 & $F(3,1042)=$ \\
& Total & 1046 & 96.27 & 0.51 & 16.47 & $13.57, p<.001$ \\
Planning & African American & 156 & $104.51^{\mathrm{a}}$ & 1.28 & 15.93 & \\
& Asian/Indian & 150 & $114.47^{\mathrm{b}}$ & 1.98 & 24.31 & \\
& Caucasian & 617 & $114.66^{\mathrm{b}}$ & 1.04 & 25.95 & \\
& Latino & 119 & $111.25^{\mathrm{a}, \mathrm{b}}$ & 1.99 & 21.67 & $F(3,1038)=$ \\
Pair & Total & 1042 & 112.72 & 0.75 & 24.23 & $7.84, p<.001$ \\
Cancellation & African American & 155 & $96.34^{\mathrm{a}}$ & 1.15 & 14.33 & \\
& Asian/Indian & 155 & $99.64^{\mathrm{a}, \mathrm{b}}$ & 1.37 & 17.12 & \\
& Caucasian & 621 & $100.42^{\mathrm{b}}$ & 0.57 & 14.30 & \\
& Latino & 120 & $97.23^{\mathrm{a}, \mathrm{b}}$ & 1.29 & 14.11 & $F(3,1047)=$ \\
& Total & 1051 & 99.34 & 0.46 & 14.80 & $4.10, p=.007$ \\
\hline
\end{tabular}

Note. WAIS-III/IV $=$ Wechsler Adult Intelligence Scale - Third/Fourth Editions. WJ III $=$ Woodcock-Johnson Tests of Cognitive Abilities - Third Edition. GIA = General Intellectual Ability. $\mathrm{M}=$ Mean. $\mathrm{SE}=$ Standard Error of the Mean. $\mathrm{SD}=$ Standard Deviation. *WJ III has a Standard Score $\mathrm{M}=100, \mathrm{SD}=15$. Means sharing the same superscript are not significantly different from each other (Scheffé's, $p<0.05$ ). 
Table 36

WAIS-III/IV Combined Dataset ANOVA Results for the WJ III Achievement Subtests by Ethnicity

\begin{tabular}{|c|c|c|c|c|c|c|}
\hline Subtest* & Gender & $n$ & $\mathrm{M}$ & $\mathrm{SE}$ & SD & $\begin{array}{c}\text { Group } \\
\text { Differences }\end{array}$ \\
\hline \multirow{5}{*}{$\begin{array}{l}\text { Letter-Word } \\
\text { Identification }\end{array}$} & African American & 170 & $97.22^{\mathrm{a}}$ & 0.87 & 11.38 & \\
\hline & Asian/Indian & 166 & $104.10^{\mathrm{b}, \mathrm{c}}$ & 0.70 & 9.05 & \\
\hline & Caucasian & 705 & $105.83^{\mathrm{c}}$ & 0.40 & 10.57 & \\
\hline & Latino & 127 & $102.18^{b}$ & 0.80 & 9.03 & $F(3,1164)=$ \\
\hline & Total & 1168 & 103.93 & 0.31 & 10.75 & $33.02, p<.001$ \\
\hline \multirow{5}{*}{$\begin{array}{l}\text { Reading } \\
\text { Fluency }\end{array}$} & African American & 154 & $95.52^{\mathrm{a}}$ & 1.17 & 14.54 & \\
\hline & Asian/Indian & 154 & $102.19^{b}$ & 1.18 & 14.65 & \\
\hline & Caucasian & 626 & $103.17^{\mathrm{b}}$ & 0.57 & 14.36 & \\
\hline & Latino & 120 & $97.63^{\mathrm{a}}$ & 1.22 & 13.35 & $F(3,1050)=$ \\
\hline & Total & 1054 & 101.28 & 0.45 & 14.59 & $14.74, p<.001$ \\
\hline \multirow[t]{5}{*}{ Calculation } & African American & 167 & $101.74^{\mathrm{a}}$ & 1.25 & 16.16 & \\
\hline & Asian/Indian & 166 & $118.43^{\mathrm{c}}$ & 1.16 & 14.96 & \\
\hline & Caucasian & 699 & $111.73^{\mathrm{b}}$ & 0.55 & 14.57 & \\
\hline & Latino & 127 & $109.08^{\mathrm{b}}$ & 1.21 & 13.64 & $F(3,1155)=$ \\
\hline & Total & 1159 & 110.96 & 0.45 & 15.45 & $37.14, p<.001$ \\
\hline \multirow[t]{5}{*}{ Math Fluency } & African American & 153 & $97.31^{\mathrm{a}}$ & 1.14 & 14.04 & \\
\hline & Asian/Indian & 154 & $107.19^{\mathrm{b}}$ & 1.33 & 16.49 & \\
\hline & Caucasian & 626 & $100.15^{\mathrm{a}}$ & 0.51 & 12.81 & \\
\hline & Latino & 120 & $96.79^{\mathrm{a}}$ & 1.29 & 14.14 & $F(3,1049)=$ \\
\hline & Total & 1053 & 100.39 & 0.43 & 14.06 & $17.95, p<.001$ \\
\hline \multirow[t]{5}{*}{ Spelling } & African American & 154 & $100.89^{\mathrm{a}}$ & 0.95 & 11.82 & \\
\hline & Asian/Indian & 153 & $108.84^{\mathrm{b}}$ & 0.77 & 9.52 & \\
\hline & Caucasian & 628 & $107.60^{\mathrm{b}}$ & 0.39 & 9.82 & \\
\hline & Latino & 120 & $105.83^{b}$ & 0.87 & 9.52 & $F(3,1051)=$ \\
\hline & Total & 1055 & 106.60 & 0.32 & 10.35 & $21.36, p<.001$ \\
\hline Writing & African American & 166 & $100.39^{\mathrm{a}}$ & 1.19 & 15.32 & \\
\hline \multirow[t]{4}{*}{ Fluency } & Asian/Indian & 167 & $108.69^{\mathrm{b}}$ & 1.17 & 15.15 & \\
\hline & Caucasian & 693 & $109.10^{\mathrm{b}}$ & 0.53 & 13.93 & \\
\hline & Latino & 126 & $105.08^{b}$ & 1.02 & 11.43 & $F(3,1148)=$ \\
\hline & Total & 1152 & 107.35 & 0.42 & 14.39 & $18.72, p<.001$ \\
\hline Passage & African American & 169 & $95.95^{\mathrm{a}}$ & 0.88 & 11.44 & \\
\hline \multirow{4}{*}{ Comprehension } & Asian/Indian & 166 & $104.69^{b, c}$ & 0.78 & 10.06 & \\
\hline & Caucasian & 704 & $107.42^{\mathrm{c}}$ & 0.37 & 9.70 & \\
\hline & Latino & 127 & $102.06^{\mathrm{b}}$ & 0.88 & 9.97 & $F(3,1162)=$ \\
\hline & Total & 1166 & 104.78 & 0.32 & 10.82 & $62.72, p<.001$ \\
\hline
\end{tabular}


Table 36

WAIS-III/IV Combined Dataset ANOVA Results for the WJ III Achievement Subtests by Ethnicity, continued

\begin{tabular}{|c|c|c|c|c|c|c|}
\hline Subtest* & Gender & $n$ & M & $\mathrm{SE}$ & SD & $\begin{array}{c}\text { Group } \\
\text { Differences }\end{array}$ \\
\hline Applied & African American & 168 & $96.85^{\mathrm{a}}$ & 0.94 & 12.16 & \\
\hline \multirow[t]{4}{*}{ Problems } & Asian/Indian & 165 & $112.35^{\mathrm{d}}$ & 0.89 & 11.43 & \\
\hline & Caucasian & 704 & $108.72^{c}$ & 0.48 & 12.67 & \\
\hline & Latino & 127 & $104.24^{\mathrm{b}}$ & 1.04 & 11.71 & $F(3,1160)=$ \\
\hline & Total & 1164 & 107.03 & 0.39 & 13.16 & $54.99, p<.001$ \\
\hline Writing & African American & 169 & $98.14^{\mathrm{a}}$ & 1.09 & 14.13 & \\
\hline \multirow[t]{4}{*}{ Samples } & Asian/Indian & 166 & $106.61^{\mathrm{b}}$ & 1.12 & 14.41 & \\
\hline & Caucasian & 705 & $112.02^{\mathrm{c}}$ & 0.60 & 15.94 & \\
\hline & Latino & 127 & $105.83^{b}$ & 1.29 & 14.56 & $F(3,1163)=$ \\
\hline & Total & 1167 & 108.57 & 0.47 & 16.09 & $40.19,<.001$ \\
\hline \multirow[t]{5}{*}{ Word Attack } & African American & 161 & $96.29^{\mathrm{a}}$ & 0.95 & 12.03 & \\
\hline & Asian/Indian & 162 & $104.30^{\mathrm{b}}$ & 0.87 & 11.08 & \\
\hline & Caucasian & 692 & $102.72^{b}$ & 0.41 & 10.85 & \\
\hline & Latino & 125 & $101.94^{\mathrm{b}}$ & 0.91 & 10.20 & $F(3,1136)=$ \\
\hline & Total & 1140 & 101.95 & 0.33 & 11.23 & $17.86, p<.001$ \\
\hline
\end{tabular}

Note. WAIS-III/IV $=$ Wechsler Adult Intelligence Scale - Third/Fourth Editions. WJ $\mathrm{III}=$ Woodcock-Johnson Tests of Achievement - Third Edition. $\mathrm{M}=$ Mean. SE $=$ Standard Error of the Mean. SD = Standard Deviation. *WJ III has a Standard Score $\mathrm{M}=100, \mathrm{SD}=15$. Means sharing the same superscript are not significantly different from each other (Scheffé's, $p<0.05$ ).

Table 37 exhibits the mean scale scores by ethnicity of the CAARS self- and other-rated scales. The Asian/Indian group scored in the highest group in all subscales except the other-rated subscales Hyperactivity/Restlessness and DSM-IV Hyperactive/ Impulsive Symptoms - both subscales in which there were no significant differences among groups. The Asian/Indian group, however, often shared the high group with other ethnic groups. In the self-rated subscales Inattention/Memory Problems, Impulsivity/ Emotional Lability, DSM-IV Inattentive Symptoms, DSM-IV ADHD Symptoms Total, and ADHD Index, Latinos shared the high group with Asians/Indians, although only in 
Inattention/Memory Problems, DSM-IV Inattentive Symptoms, and DSM-IV ADHD

Symptoms Total did the mean scores approach or exceed clinical significance (i.e., $\geq 70$ ).

Mean scores in the "At Risk" category (60-69) included the middle and high groups of the self-rated Inattention/Memory Problems and ADHD Index subscales.

African Americans scored consistently in the lowest groups; that is, they generally rated themselves and were rated by others with the fewest ADHD symptoms of all ethnic groups. Although African Americans shared this low group with other ethnic groups in many of the other-rated scales, their scores were uniquely low in six of the eight selfrated subscales. Only on the self-rated subscales of Hyperactivity/ Restlessness and Impulsivity/Emotional Lability did African Americans share the low group with Caucasians. Again, the Latino group typically scored in the middle groups, often also sharing the high group with the Asian/Indian group, such as in the self-rated subtests Inattention/Memory Problems, Impulsivity/Emotional Lability, DSM-IV Inattentive Symptoms, DSM-IV ADHD Symptoms Total, and ADHD Index. Neither Caucasians nor Latinos singularly represented any group, but shared either the low group with the African Americans or the high group with the Asians/Indians. There were no significant differences between the Caucasian and Latino groups on any of the self- or other-rated CAARS subscales. 
Table 37

WAIS-III/IV Combined Dataset ANOVA Results for the CAARS Self-and Other-rated Scales by Ethnicity

\begin{tabular}{|c|c|c|c|c|c|c|}
\hline Scale* & Ethnicity & $n$ & M & $\mathrm{SE}$ & SD & $\begin{array}{c}\text { Group } \\
\text { Differences }\end{array}$ \\
\hline $\begin{array}{l}\text { S-CAARS A } \\
\text { Inattention/ } \\
\text { Memory } \\
\text { Problems }\end{array}$ & $\begin{array}{l}\text { African American } \\
\text { Asian/Indian } \\
\text { Caucasian } \\
\text { Latino } \\
\text { Total }\end{array}$ & $\begin{array}{r}173 \\
169 \\
705 \\
130 \\
1177\end{array}$ & $\begin{array}{l}57.51^{\mathrm{a}} \\
68.15^{\mathrm{c}} \\
64.03^{\mathrm{b}} \\
65.71^{\mathrm{b}, \mathrm{c}} \\
63.85\end{array}$ & $\begin{array}{l}1.16 \\
0.91 \\
0.50 \\
1.06 \\
0.39\end{array}$ & $\begin{array}{l}15.31 \\
11.84 \\
13.16 \\
12.04 \\
13.52\end{array}$ & $\begin{array}{c}F(3,1173)= \\
20.15, p<.001\end{array}$ \\
\hline $\begin{array}{l}\text { S-CAARS B } \\
\text { Hyperactivity/ } \\
\text { Restlessness }\end{array}$ & $\begin{array}{l}\text { African American } \\
\text { Asian/Indian } \\
\text { Caucasian } \\
\text { Latino } \\
\text { Total }\end{array}$ & $\begin{array}{r}173 \\
169 \\
705 \\
130 \\
1177\end{array}$ & $\begin{array}{l}52.51^{\mathrm{a}} \\
57.40^{\mathrm{b}} \\
55.15^{\mathrm{a}, \mathrm{b}} \\
56.29^{\mathrm{b}} \\
55.21\end{array}$ & $\begin{array}{l}0.99 \\
0.89 \\
0.46 \\
1.02 \\
0.35\end{array}$ & $\begin{array}{l}13.03 \\
11.58 \\
12.10 \\
11.62 \\
12.18\end{array}$ & $\begin{array}{c}F(3,1173)= \\
5.05, p=.002\end{array}$ \\
\hline $\begin{array}{l}\text { S-CAARS C } \\
\text { Impulsivity/ } \\
\text { Emotional } \\
\text { Lability }\end{array}$ & $\begin{array}{l}\text { African American } \\
\text { Asian/Indian } \\
\text { Caucasian } \\
\text { Latino } \\
\text { Total }\end{array}$ & $\begin{array}{r}173 \\
169 \\
705 \\
130 \\
1177\end{array}$ & $\begin{array}{l}49.75^{\mathrm{a}} \\
56.78^{\mathrm{c}} \\
53.09^{\mathrm{a}, \mathrm{b}} \\
54.28^{\mathrm{b}, \mathrm{c}} \\
53.26\end{array}$ & $\begin{array}{l}1.02 \\
0.95 \\
0.46 \\
1.07 \\
0.37\end{array}$ & & $\begin{array}{c}F(3,1173)= \\
9.34, p<.001\end{array}$ \\
\hline $\begin{array}{l}\text { S-CAARS D } \\
\text { Problems } \\
\text { with Self } \\
\text { Concept }\end{array}$ & $\begin{array}{l}\text { African American } \\
\text { Asian/Indian } \\
\text { Caucasian } \\
\text { Latino } \\
\text { Total }\end{array}$ & $\begin{array}{r}173 \\
169 \\
705 \\
130 \\
1177\end{array}$ & $\begin{array}{l}50.47^{\mathrm{a}} \\
59.74^{\mathrm{c}} \\
55.27^{\mathrm{b}} \\
55.95^{\mathrm{b}} \\
55.28\end{array}$ & $\begin{array}{l}0.94 \\
0.89 \\
0.44 \\
0.97 \\
0.35\end{array}$ & $\begin{array}{l}12.35 \\
11.54 \\
11.71 \\
11.04 \\
11.96\end{array}$ & $\begin{array}{c}F(3,1173)= \\
18.03, p<.001\end{array}$ \\
\hline $\begin{array}{l}\text { S-CAARS E } \\
\text { DSM-IV } \\
\text { Inattentive } \\
\text { Symptoms }\end{array}$ & $\begin{array}{l}\text { African Americar } \\
\text { Asian/Indian } \\
\text { Caucasian } \\
\text { Latino } \\
\text { Total }\end{array}$ & $\begin{array}{r}173 \\
169 \\
705 \\
130 \\
1177\end{array}$ & $\begin{array}{l}65.58^{\mathrm{a}} \\
77.75^{\mathrm{c}} \\
73.14^{\mathrm{b}} \\
74.96^{\mathrm{b}, \mathrm{c}} \\
72.89\end{array}$ & $\begin{array}{l}1.34 \\
1.01 \\
0.55 \\
1.22 \\
0.44\end{array}$ & $\begin{array}{l}17.61 \\
13.19 \\
14.56 \\
13.86 \\
15.16\end{array}$ & $\begin{array}{c}F(3,1173)= \\
21.11, p<.001\end{array}$ \\
\hline $\begin{array}{l}\text { S-CAARS F } \\
\text { DSM-IV } \\
\text { Hyperactive/ } \\
\text { Impulsive } \\
\text { Symptoms }\end{array}$ & $\begin{array}{l}\text { African American } \\
\text { Asian/Indian } \\
\text { Caucasian } \\
\text { Latino } \\
\text { Total }\end{array}$ & $\begin{array}{r}173 \\
169 \\
704 \\
130 \\
1176\end{array}$ & $\begin{array}{l}53.37^{\mathrm{a}} \\
60.59^{\mathrm{b}} \\
57.86^{\mathrm{b}} \\
59.95^{\mathrm{b}} \\
57.82\end{array}$ & $\begin{array}{l}1.24 \\
1.17 \\
0.57 \\
1.30 \\
0.45\end{array}$ & $\begin{array}{l}16.33 \\
15.20 \\
15.18 \\
14.86 \\
15.45\end{array}$ & $\begin{array}{c}F(3,1172)= \\
7.54, p<.001\end{array}$ \\
\hline $\begin{array}{l}\text { S-CAARS G } \\
\text { DSM-IV } \\
\text { ADHD } \\
\text { Symptoms } \\
\text { Total }\end{array}$ & $\begin{array}{l}\text { African American } \\
\text { Asian/Indian } \\
\text { Caucasian } \\
\text { Latino } \\
\text { Total }\end{array}$ & $\begin{array}{r}173 \\
169 \\
704 \\
130 \\
1176\end{array}$ & $\begin{array}{l}61.42^{\mathrm{a}} \\
73.34^{\mathrm{c}} \\
68.79^{\mathrm{b}} \\
71.21^{\mathrm{b}, \mathrm{c}} \\
68.63\end{array}$ & $\begin{array}{l}1.35 \\
1.11 \\
0.56 \\
1.30 \\
0.46\end{array}$ & $\begin{array}{l}17.77 \\
14.41 \\
14.97 \\
14.80 \\
15.67\end{array}$ & $\begin{array}{c}F(3,1172)= \\
19.34, p<.001\end{array}$ \\
\hline
\end{tabular}


Table 37

WAIS-III/IV Combined Dataset ANOVA Results for the CAARS Self- and Other-rated Scales by Ethnicity, continued

\begin{tabular}{|c|c|c|c|c|c|c|}
\hline Scale* & Ethnicity & $n$ & $\mathrm{M}$ & SE & SD & $\begin{array}{c}\text { Group } \\
\text { Differences }\end{array}$ \\
\hline $\begin{array}{l}\text { S-CAARS H } \\
\text { ADHD } \\
\text { Index }\end{array}$ & $\begin{array}{l}\text { African American } \\
\text { Asian/Indian } \\
\text { Caucasian } \\
\text { Latino } \\
\text { Total }\end{array}$ & $\begin{array}{r}173 \\
169 \\
704 \\
130 \\
1176\end{array}$ & $\begin{array}{l}53.90^{\mathrm{a}} \\
63.98^{\mathrm{c}} \\
59.39^{\mathrm{b}} \\
61.13^{\mathrm{b}, \mathrm{c}} \\
59.43\end{array}$ & $\begin{array}{l}1.04 \\
0.78 \\
0.42 \\
0.89 \\
0.34\end{array}$ & $\begin{array}{l}13.63 \\
10.13 \\
11.01 \\
10.12 \\
11.55\end{array}$ & $\begin{array}{c}F(3,1172)= \\
24.23, p<.001\end{array}$ \\
\hline $\begin{array}{l}\text { O-CAARS A } \\
\text { Inattention/ } \\
\text { Memory } \\
\text { Problems }\end{array}$ & $\begin{array}{l}\text { African American } \\
\text { Asian/Indian } \\
\text { Caucasian } \\
\text { Latino } \\
\text { Total }\end{array}$ & $\begin{array}{r}82 \\
107 \\
456 \\
82 \\
727\end{array}$ & $\begin{array}{l}57.28^{\mathrm{a}} \\
66.13^{\mathrm{b}} \\
61.26^{\mathrm{a}, \mathrm{b}} \\
61.72^{\mathrm{a}, \mathrm{b}} \\
61.58\end{array}$ & $\begin{array}{l}1.58 \\
1.25 \\
0.65 \\
1.50 \\
0.52 \\
\end{array}$ & $\begin{array}{l}14.35 \\
12.95 \\
13.95 \\
13.60 \\
13.97\end{array}$ & $\begin{array}{c}F(3,723)= \\
6.60, p<.001\end{array}$ \\
\hline $\begin{array}{l}\text { O-CAARS B } \\
\text { Hyperactivity/ } \\
\text { Restlessness }\end{array}$ & $\begin{array}{l}\text { African American } \\
\text { Asian/Indian } \\
\text { Caucasian } \\
\text { Latino } \\
\text { Total }\end{array}$ & $\begin{array}{r}82 \\
107 \\
456 \\
82 \\
727\end{array}$ & $\begin{array}{l}53.30 \\
56.96 \\
54.23 \\
55.12 \\
54.63\end{array}$ & $\begin{array}{l}1.60 \\
1.27 \\
0.60 \\
1.41 \\
0.48\end{array}$ & $\begin{array}{l}14.47 \\
13.15 \\
12.75 \\
12.76 \\
13.03\end{array}$ & $\begin{array}{c}F(3,723)= \\
1.629, p=.184\end{array}$ \\
\hline $\begin{array}{l}\text { O-CAARS C } \\
\text { Impulsivity/ } \\
\text { Emotional } \\
\text { Lability }\end{array}$ & $\begin{array}{l}\text { African American } \\
\text { Asian/Indian } \\
\text { Caucasian } \\
\text { Latino } \\
\text { Total }\end{array}$ & $\begin{array}{r}82 \\
107 \\
456 \\
82 \\
727\end{array}$ & $\begin{array}{l}50.17^{\mathrm{a}} \\
54.95^{\mathrm{b}} \\
51.79^{\mathrm{a}, \mathrm{b}} \\
54.06^{\mathrm{a}, \mathrm{b}} \\
52.33\end{array}$ & $\begin{array}{l}1.24 \\
1.10 \\
0.51 \\
1.25 \\
0.41\end{array}$ & $\begin{array}{l}11.21 \\
11.39 \\
10.79 \\
11.36 \\
11.07\end{array}$ & $\begin{array}{c}F(3,723)= \\
4.13, p=.006\end{array}$ \\
\hline $\begin{array}{l}\text { O-CAARS D } \\
\text { Problems } \\
\text { with Self } \\
\text { Concept }\end{array}$ & $\begin{array}{l}\text { African American } \\
\text { Asian/Indian } \\
\text { Caucasian } \\
\text { Latino } \\
\text { Total }\end{array}$ & $\begin{array}{r}82 \\
107 \\
456 \\
82 \\
727\end{array}$ & $\begin{array}{l}52.73^{\mathrm{a}} \\
57.93^{\mathrm{b}} \\
54.62^{\mathrm{a}, \mathrm{b}} \\
53.65^{\mathrm{a} \cdot \mathrm{b}} \\
54.79\end{array}$ & $\begin{array}{l}1.41 \\
1.14 \\
0.54 \\
1.31 \\
0.44 \\
\end{array}$ & $\begin{array}{l}12.74 \\
11.78 \\
11.63 \\
11.84 \\
11.87\end{array}$ & $\begin{array}{c}F(3,723)= \\
3.65, p=.012\end{array}$ \\
\hline $\begin{array}{l}\text { O-CAARS E } \\
\text { DSM-IV } \\
\text { Inattentive } \\
\text { Symptoms }\end{array}$ & $\begin{array}{l}\text { African American } \\
\text { Asian/Indian } \\
\text { Caucasian } \\
\text { Latino } \\
\text { Total }\end{array}$ & $\begin{array}{r}82 \\
107 \\
456 \\
82 \\
727\end{array}$ & $\begin{array}{l}57.71^{\mathrm{a}} \\
64.79^{\mathrm{b}} \\
60.86^{\mathrm{a}, \mathrm{b}} \\
61.82^{\mathrm{a}, \mathrm{b}} \\
61.19\end{array}$ & $\begin{array}{l}1.51 \\
1.19 \\
0.58 \\
1.37 \\
0.47 \\
\end{array}$ & $\begin{array}{l}13.71 \\
12.30 \\
12.43 \\
12.37 \\
12.66\end{array}$ & $\begin{array}{c}F(3,723)= \\
5.20, p=.001\end{array}$ \\
\hline $\begin{array}{l}\text { O-CAARS F } \\
\text { DSM-IV } \\
\text { Hyperactive/ } \\
\text { Impulsive } \\
\text { Symptoms }\end{array}$ & $\begin{array}{l}\text { African American } \\
\text { Asian/Indian } \\
\text { Caucasian } \\
\text { Latino } \\
\text { Total }\end{array}$ & $\begin{array}{r}82 \\
108 \\
456 \\
82 \\
728\end{array}$ & $\begin{array}{l}53.37 \\
56.31 \\
54.57 \\
56.38 \\
54.90\end{array}$ & $\begin{array}{l}1.36 \\
1.35 \\
0.60 \\
1.54 \\
0.49\end{array}$ & $\begin{array}{l}12.30 \\
14.03 \\
12.87 \\
13.98 \\
13.12\end{array}$ & $\begin{array}{c}F(3,724)= \\
1.23, p=.297\end{array}$ \\
\hline
\end{tabular}


Table 37

WAIS-III/IV Combined Dataset ANOVA Results for the CAARS Self-and Other-rated Scales by Ethnicity, continued

\begin{tabular}{|c|c|c|c|c|c|c|}
\hline Scale* & Ethnicity & $n$ & M & $\mathrm{SE}$ & SD & $\begin{array}{c}\text { Group } \\
\text { Differences }\end{array}$ \\
\hline O-CAARS G & African American & 82 & $56.62^{\mathrm{a}}$ & 1.48 & 13.41 & \\
\hline DSM-IV & Asian/Indian & 107 & $62.44^{\mathrm{b}}$ & 1.21 & 12.50 & \\
\hline ADHD & Caucasian & 456 & $59.10^{\mathrm{a}, \mathrm{b}}$ & 0.59 & 12.61 & \\
\hline Symptoms & Latino & 82 & $60.73^{a, b}$ & 1.49 & 13.50 & $F(3,723)=$ \\
\hline Total & Total & 727 & 59.50 & 0.48 & 12.86 & $3.67, p=.012$ \\
\hline O-CAARS H & African American & 82 & $56.30^{\mathrm{a}}$ & 1.47 & 13.31 & \\
\hline ADHD & Asian/Indian & 107 & $61.75^{c}$ & 1.11 & 11.51 & \\
\hline \multirow[t]{3}{*}{ Index } & Caucasian & 456 & $57.97^{\mathrm{a}, \mathrm{b}}$ & 0.54 & 11.52 & \\
\hline & Latino & 82 & $59.09^{\mathrm{a}, \mathrm{b}}$ & 1.35 & 12.24 & $F(3,723)=$ \\
\hline & Total & 727 & 58.47 & 0.44 & 11.89 & $4.01, p=.008$ \\
\hline
\end{tabular}

Note. WAIS-III/IV = Wechsler Adult Intelligence Scale - Third/Fourth Editions. CAARS $=$ Conners' Adult ADHD Rating Scale. $\mathrm{M}=$ Mean. $\mathrm{SE}=$ Standard Error of the Mean. SD = Standard Deviation. $\mathrm{S}=$ Self-Rated Scale. $\mathrm{O}=$ Other-Rated Scale. *CAARS Rating Scales have a T-score $\mathrm{M}=50, \mathrm{SD}=10$. Means sharing the same superscript are not significantly different from each other (Scheffé's, $p<0.05$ ).

Table 38 illustrates the mean score differences on the BDI-II and SCL-90-R. On the BDI-II, the Asian/Indian group scored significantly higher than the Caucasian and African American groups, meaning Asian/Indian students expressed more depressive symptoms than the other two ethnic groups. The Latino group shared the high group with the Asians/Indian group, but its scores were not significantly different from the African American or Caucasian group scores. A similar scoring scenario occurred with the SCL90-R: the Asian/Indian group scored significantly higher than the African American and Caucasian groups, and the Latino group scored similarly to the other three groups. 
Table 38

WAIS-III/IV Combined Dataset ANOVA Results for the BDI-II Total Score and SCL90-R GSI by Ethnicity

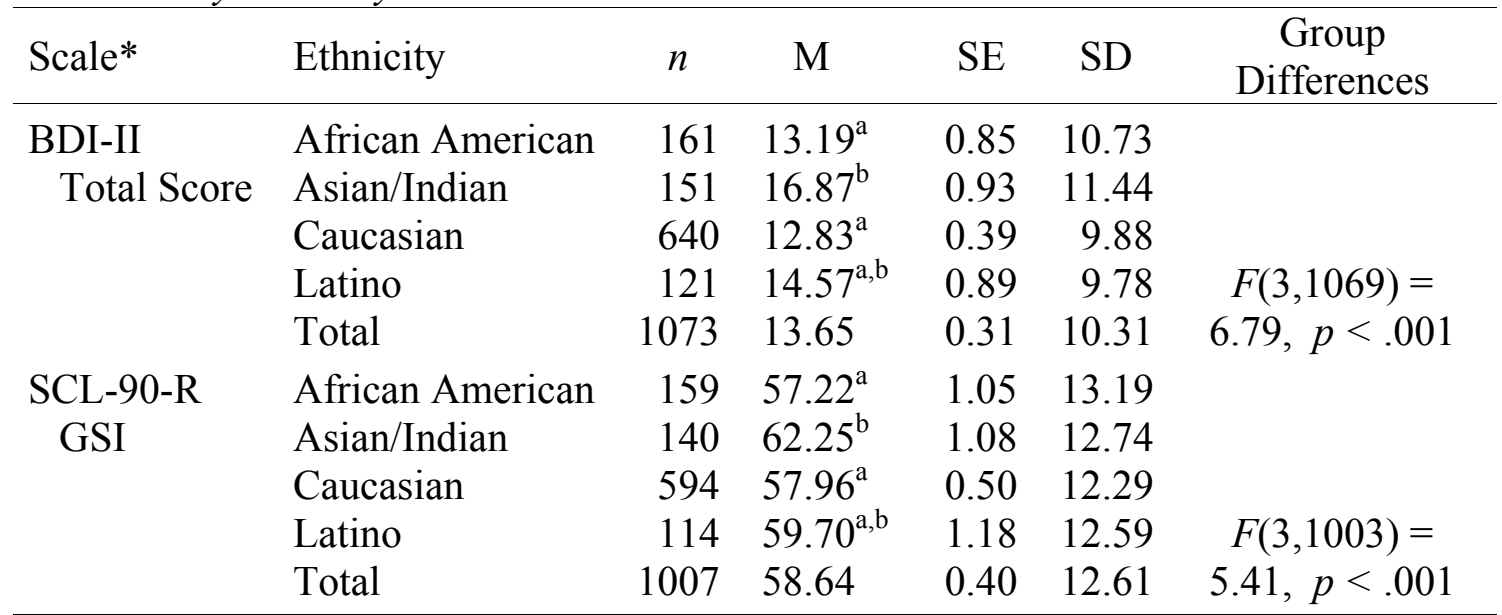

Note. WAIS-III/IV = Wechsler Adult Intelligence Scale - Third/Fourth Editions.

BDI-II = Beck Depression Inventory - Second Edition. SCL-90-R GSI = Symptom Checklist-90-Revised Global Severity Index. $\mathrm{M}=$ Mean. $\mathrm{SE}=$ Standard Error of the Mean. SD = Standard Deviation. *Cut score guidelines for the BDI-II Total Score: 013 is considered minimal range, $14-19$ is mild, 20-28 is moderate, and 29-63 is severe for symptoms of depression; the GSI has a T-score $\mathrm{M}=50, \mathrm{SD}=10$. Means sharing the same superscript are not significantly different from each other (Scheffé's, $p<0.05$ ).

Table 39 represents a cross-tabulation chi square analysis of diagnosis by ethnicity, as Table 33 did for diagnosis by gender. Diagnosis was compared to No Diagnosis, and Primary Diagnosis was compared to Secondary Diagnosis by ethnicity for only the diagnoses that showed significant differences. There was no evidence of significant differences among ethnic groups in the proportional rates of diagnosis for either depression or anxiety, or for primary versus secondary diagnoses of those two disorders.

Consistent with the CAARS scores, a smaller proportion of African Americans were diagnosed with ADHD-Combined (ADHD-C) than were any of the other ethnic groups. Although this ratio also held true for the primary diagnosis of ADHD-C, there 
was no evidence of significant differences in the proportion of secondary diagnoses of ADHD-C. Regarding ADHD-Inattentive (ADHD-I), the proportion of African American diagnoses were fewer than the proportion of Asian/Indian diagnoses, but there was no evidence of significant differences between these two groups and the Caucasian and Latino groups. This relation again held for the primary diagnosis of ADHD-I but not for the secondary diagnosis, in which, again, there was no evidence of significant differences among groups.

For Verbal Learning Disability (VLD), African Americans were diagnosed in proportionally higher numbers than Caucasians, who, in turn, were diagnosed at a higher rate than Asians/Indians. Latinos were diagnosed at a rate that fell between Caucasians and Asians/Indians, but there was no evidence of significant differences between Latinos' proportion of diagnoses and the proportions of Caucasian or Asian/Indian diagnoses. Significant differences were found for the proportion of Nonverbal Learning Disability (NVLD) only between African Americans, who obtained the highest proportion of diagnoses, and Latinos, who had the lowest proportion. The proportions of NVLD diagnoses for Caucasians and Asians/Indians did not differ significantly from the proportions of the other two groups. Similar proportions were found for the Primary diagnosis of NVLD, but for Secondary NVLD, only Latinos differed from the other three groups with a lower proportion of Latinos diagnosed with NVLD. Sample size was extremely small, however, making statistical comparisons potentially invalid. 
Table 39

Cross-tabulations of Diagnosis and Ethnicity from the WAIS-III/IV Combined Dataset

\begin{tabular}{|c|c|c|c|c|c|c|c|c|c|c|c|c|c|c|}
\hline \multicolumn{2}{|c|}{ Diagnosis } & \multicolumn{8}{|c|}{ Ethnicity } & \multirow[t]{3}{*}{ Total } & \multirow[t]{3}{*}{$\%$} & \multirow[t]{3}{*}{$\chi^{2}$} & \multirow[t]{3}{*}{$\mathrm{df}$} & \multirow[t]{3}{*}{$p$} \\
\hline & & \multicolumn{2}{|c|}{$\begin{array}{c}\text { African } \\
\text { American } \\
\end{array}$} & \multicolumn{2}{|c|}{$\begin{array}{l}\text { Asian/ } \\
\text { Indian }\end{array}$} & \multicolumn{2}{|c|}{$\underline{\text { Caucasian }}$} & \multicolumn{2}{|c|}{$\underline{\text { Latino }}$} & & & & & \\
\hline & & $n$ & $\%$ & $n$ & $\%$ & $n$ & $\%$ & $n$ & $\%$ & & & & & \\
\hline \multirow[t]{4}{*}{ ADHD-C } & No & $146^{\mathrm{a}}$ & 76.4 & $109^{\mathrm{b}}$ & 59.6 & $501^{\mathrm{b}}$ & 64.3 & $77^{\mathrm{b}}$ & 57.9 & 833 & 64.8 & & & \\
\hline & Yes & $45^{\mathrm{a}}$ & 23.6 & $74^{\mathrm{b}}$ & 40.4 & $278^{\mathrm{b}}$ & 35.7 & $56^{\mathrm{b}}$ & 42.1 & 453 & 35.2 & 16.40 & 3 & $<.001$ \\
\hline & Primary & $33^{\mathrm{a}}$ & 17.3 & $62^{\mathrm{b}}$ & 33.9 & $241^{b}$ & 30.9 & $47^{\mathrm{b}}$ & 35.3 & 383 & 29.8 & & & \\
\hline & Secondary & $12^{\mathrm{a}}$ & 6.3 & $12^{\mathrm{a}}$ & 6.6 & $37^{\mathrm{a}}$ & 4.7 & $9^{\mathrm{a}}$ & 5.4 & 70 & 5.4 & 20.35 & 6 & .002 \\
\hline \multirow[t]{4}{*}{ ADHD-I } & No & $156^{\mathrm{a}}$ & 81.7 & $128^{\mathrm{b}}$ & 69.9 & $592^{\mathrm{a}, \mathrm{b}}$ & 76.0 & $96^{\mathrm{a}, \mathrm{b}}$ & 72.2 & 972 & 75.6 & & & \\
\hline & Yes & $35^{\mathrm{a}}$ & 18.3 & $55^{\mathrm{b}}$ & 30.1 & $187^{\mathrm{a}, \mathrm{b}}$ & 24.0 & $37^{\mathrm{a}, \mathrm{b}}$ & 27.8 & 314 & 24.4 & 7.90 & 3 & .048 \\
\hline & Primary & $28^{\mathrm{a}}$ & 14.7 & $53^{\mathrm{b}}$ & 29.0 & $173^{\mathrm{a}, \mathrm{b}}$ & 22.2 & $34^{\mathrm{a}, \mathrm{b}}$ & 25.6 & 288 & 22.4 & & & \\
\hline & Secondary & $7^{\mathrm{a}}$ & 3.7 & $2^{\mathrm{a}}$ & 1.1 & $14^{\mathrm{a}}$ & 1.8 & $3^{\mathrm{a}}$ & 2.3 & 26 & 2.0 & 14.73 & 6 & .022 \\
\hline \multirow[t]{4}{*}{ VLD } & No & $135^{\mathrm{a}}$ & 70.7 & $173^{b}$ & 94.5 & $659^{c}$ & 84.6 & $117^{\mathrm{b}, \mathrm{c}}$ & 88.0 & 1084 & 84.3 & & & \\
\hline & Yes & $56^{\mathrm{a}}$ & 29.3 & $10^{\mathrm{b}}$ & 5.5 & $120^{\mathrm{c}}$ & 15.4 & $16^{\mathrm{b}, \mathrm{c}}$ & 12.0 & 202 & 15.7 & 42.64 & 3 & $<.001$ \\
\hline & Primary & $54^{\mathrm{a}}$ & 70.7 & $7^{\mathrm{b}}$ & 3.8 & $105^{\mathrm{c}}$ & 13.5 & $15^{\mathrm{b}, \mathrm{c}}$ & 11.3 & 181 & 14.1 & & & \\
\hline & Secondary & $2^{\mathrm{a}}$ & 1.0 & $3^{\mathrm{a}}$ & 1.6 & $15^{\mathrm{a}}$ & 1.9 & $1^{\mathrm{a}}$ & 0.8 & 21 & 1.6 & 50.09 & 6 & $<.001$ \\
\hline \multirow[t]{4}{*}{ NVLD } & No & $160^{\mathrm{a}}$ & 83.8 & $167^{\mathrm{a}, \mathrm{b}}$ & 91.3 & $695^{\mathrm{a}, \mathrm{b}}$ & 89.2 & $127^{\mathrm{b}}$ & 95.5 & 1149 & 89.3 & & & \\
\hline & Yes & $31^{\mathrm{a}}$ & 16.2 & $16^{\mathrm{a}, \mathrm{b}}$ & 8.7 & $84^{\mathrm{a}, \mathrm{b}}$ & 10.8 & $6^{\mathrm{b}}$ & 4.5 & 137 & 10.7 & 12.23 & 3 & .007 \\
\hline & Primary & $28^{\mathrm{a}}$ & 14.7 & $13^{\mathrm{a}, \mathrm{b}}$ & 7.1 & $69^{\mathrm{a}, \mathrm{b}}$ & 8.9 & $5^{b}$ & 3.8 & 115 & 8.9 & & & \\
\hline & Secondary & $3^{\mathrm{a}}$ & 1.6 & $3^{\mathrm{a}}$ & 1.6 & $15^{\mathrm{a}}$ & 1.9 & $1^{b}$ & 0.8 & 22 & 1.7 & 13.93 & 6 & .030 \\
\hline Total & & 191 & 100.0 & 183 & 100.0 & 779 & 100.0 & 133 & 100.0 & 1286 & 100.0 & & & \\
\hline
\end{tabular}


Table 39

Cross-tabulations of Diagnosis and Ethnicity from the WAIS-III/IV Combined Dataset, continued

Notes: $\%=$ percentage of total students in the dataset. Table compares numbers of students receiving diagnosis with numbers of students not receiving diagnoses, and numbers of students receiving a primary versus secondary diagnosis. Each superscript letter denotes a subset of Ethnicity categories whose column proportions do not significantly differ from each other at the .05 level. ADHD-C = Attention Deficit Hyperactivity Disorder - Combined Type; ADHD-I = Attention Deficit Hyperactivity Disorder - Inattentive Type, VLD=Verbal Learning Disability; NVLD=Nonverbal Learning Disability. 


\section{Primary Diagnosis}

The mean scores on all batteries were examined for differences by primary diagnosis. Tables $40-44$ illustrate the results of this examination. Only the top six diagnoses and "No Diagnosis" were considered for purposes of this examination. Of the cognitive and achievement batteries, only four subtests demonstrated no significant main effect of diagnosis: the fluid reasoning subtest Figure Weights [perhaps because of small sample size, particularly for the diagnoses of Anxiety, Depression, Nonverbal Learning Disability (NVLD), and verbal learning disability (VLD)], the visual-spatial processing subtest Picture Recognition, which trended toward significance, $(F 6,1094)=1.97, p=$ .068 , and the processing speed subtests Retrieval Fluency, Rapid Picture Naming, and Cancellation (again, perhaps because of small total sample size, $N=65$, no scores for Depression, and only one score for Anxiety). Four additional subtests exhibited main effects for diagnosis but no individual effects after analyses with the more stringent Scheffé post hoc tests: the visual-spatial processing subtests Picture Recognition, Picture Arrangement and Visual Puzzles (perhaps also because of low sample sizes in several of the cells), and the auditory processing subtest Incomplete Words.

Perhaps surprisingly, students receiving No Diagnosis were not in the high group of mean WAIS-III/IV FSIQ scores. That distinction came to students diagnosed with Depression: The mean FSIQ scores of students diagnosed with Depression shared the highest of three groups with the mean FSIQ scores of students diagnosed with ADHDPredominantly Inattentive Type (ADHD-I). The ADHD-I group also shared the middle group with the similarly scored No Diagnosis, ADHD - Predominantly Combined Type 
(ADHD-C), Anxiety, and VLD groups. As with the FSIQ, the Depression and ADHD-I groups shared the highest of two groups for GIA, but their scores differed only from the mean GIA scores of students diagnosed with VLD and NVLD. The Depression group's GIA scores did not significantly differ from those of the No Diagnosis, ADHD-C, or Anxiety groups. In turn, the VLD and NVLD groups shared the low group, but their GIA mean scores also did not differ significantly from scores from the ADHD-C, Anxiety, and No Diagnosis groups.

No diagnostic group singularly outperformed any other diagnostic group on any cognitive or achievement subtest. However, the Depression diagnostic group maintained its position in the highest group on every cognitive and achievement subtest for which significant differences were detected, although its scores were often similar to scores of several other diagnostic groups. This finding contradicts Francomano and colleagues' (2011) research suggesting that students with depression perform more poorly than their non-affected peers on cognitive tests. These findings did not translate to enhanced achievement measured by GPA, however. Although an analysis of variance indicated significant differences among the diagnostic groups on pre-test GPA $(F(6,944)=2.48, p$ $=.022)$, post hoc comparisons using a Bonferroni correction did not indicate a significant difference between the Depression group's GPA and any other group ( $p=1.00)$.

Predictably, the VLD diagnosis portended relatively low mean scores on all the verbal comprehension-knowledge subtests, but the VLD group's mean scores were significantly dissimilar from the scores of all other diagnostic groups on only two cognitive subtests: the comprehension-knowledge subtest Vocabulary and, interestingly, 
the auditory processing subtest Sound Blending. Students diagnosed with VLD appeared to have greater difficulty synthesizing discrete language sounds than the other diagnostic groups did. The VLD diagnosis also seems to have the most deleterious effect of all diagnoses on achievement. Particularly, the VLD group found itself alone in the low group of mean scores on the reading and writing knowledge (Grw) subtests Letter-Word Identification, Passage Comprehension, Reading Fluency, Spelling, and Word Attack. There was no evidence of significant differences in the mean scores of any of the other diagnostic groups (including the No Diagnosis group) on these Grw subtests. The analysis of variance detecting pre-GPA differences among the diagnostic groups $(F(6$, $944)=2.48, p=.022)$ indicated in Bonferroni post hoc analyses that, although the VLD group obtained the lowest GPAs at pre-test of all diagnostic groups, their GPAs differed significantly only from the GPAs of the ADHD-Inattentive group $(p=.039)$. There was no evidence of significant differences in pre-test GPAs between the VLD group and any other group besides the ADHD-Inattentive group, or among any of the other groups.

The NVLD diagnosis also predicted difficulty on several cognitive and achievement subtests. Predictably, students diagnosed with NVLD scored lower than any other group on the visual-spatial processing subtests Picture Completion, Block Design, and Spatial Relations; and on the fluid reasoning subtest Matrix Reasoning. There was no evidence that the other groups differed from each other on their mean scores of these subtests. The NVLD group also obtained the lowest scores on the long-term storage and retrieval subtest Visual-Auditory Learning and the processing speed subtest Symbol Search, but their scores differed significantly only from the scores of the Depression 
group on these two subtests. Although the NVLD diagnosis predicted being in the lowest group in the quantitative knowledge subtests Calculation, Arithmetic, and Applied Problems; the fluid reasoning subtests Concept Formation and Analysis-Synthesis; the visual-spatial processing subtests Visual Matching and Planning; the working-memory capacity subtests Digit Span and Letter-Number Sequencing; and the processing speed subtests Coding, Symbol Search, Decision Speed, and Pair Cancellation, NVLD mean scores were not dissimilar to those of several other diagnostic groups on these subtests. The NVLD group fared as well as all diagnostic groups, and better than the VLD group, on the comprehension-knowledge subtests; they scored in the highest group on Vocabulary. Similar results are exhibited on the reading and writing knowledge achievement subtests, with NVLD group mean scores above the VLD group mean scores on all reading and writing knowledge subtests except Writing Fluency and Writing Samples, in which the NVLD mean scores were similar to the scores of all diagnostic groups.

The No Diagnosis, ADHD-C, ADHD-I, and Anxiety groups exhibited few differences in cognitive or achievement subtest scores. These four groups typically found themselves in the "middle ground": on par with the Depression group but often not dissimilar to the VLD or NVLD groups. Some differences include: Verbal Comprehension (all groups similar except the VLD group, which shared the low group with the No Diagnosis and Anxiety groups), Digit Span (the ADHD-C group shared the low group with all other diagnoses except Depression), and Calculation (the No Diagnosis group differed significantly from the high group, which was shared by all 
diagnoses except VLD and NVLD).

Table 40

WAIS-III/IV Combined Dataset ANOVA Results for WAIS-III/IV FSIQ and Subtests by Primary Diagnosis

\begin{tabular}{|c|c|c|c|c|c|c|}
\hline $\begin{array}{l}\text { FSIQ*/ } \\
\text { Subtest** }\end{array}$ & Diagnosis & $n$ & M & $\mathrm{SE}$ & SD & $\begin{array}{c}\text { Group } \\
\text { Differences }\end{array}$ \\
\hline \multirow[t]{8}{*}{ FSIQ } & No Diagnosis & 131 & $111.62^{\mathrm{a}, \mathrm{b}}$ & 1.22 & 13.99 & \\
\hline & ADHD-C & 363 & $113.02^{\mathrm{a}, \mathrm{b}}$ & 0.67 & 12.74 & \\
\hline & ADHD-I & 283 & $115.53^{b, c}$ & 0.78 & 13.08 & \\
\hline & Anxiety & 60 & $113.15^{\mathrm{a}, \mathrm{b}}$ & 1.56 & 12.07 & \\
\hline & Depression & 39 & $120.33^{c}$ & 2.49 & 15.56 & \\
\hline & NVLD & 103 & $108.40^{\mathrm{a}}$ & 1.41 & 14.31 & \\
\hline & VLD & 141 & $108.71^{\mathrm{a}, \mathrm{b}}$ & 1.14 & 13.51 & $F(6,1113)=$ \\
\hline & Total & 1120 & 112.79 & 0.41 & 13.55 & $8.39, p<.001$ \\
\hline \multirow[t]{8}{*}{ Vocabulary } & No Diagnosis & 131 & $12.61^{\mathrm{b}}$ & 0.26 & 3.01 & \\
\hline & ADHD-C & 363 & $13.07^{\mathrm{b}, \mathrm{c}}$ & 0.15 & 2.89 & \\
\hline & ADHD-I & 283 & $13.45^{b, c}$ & 0.18 & 3.08 & \\
\hline & Anxiety & 60 & $12.93^{\mathrm{b}, \mathrm{c}}$ & 0.36 & 2.75 & \\
\hline & Depression & 40 & $13.20^{\mathrm{b}, \mathrm{c}}$ & 0.56 & 3.53 & \\
\hline & NVLD & 103 & $14.40^{\mathrm{c}}$ & 0.28 & 2.81 & \\
\hline & VLD & 141 & $11.05^{\mathrm{a}}$ & 0.24 & 2.82 & $F(6,1114)=$ \\
\hline & Total & 1121 & 12.98 & 0.09 & 3.07 & $15.62, p<.001$ \\
\hline \multirow[t]{8}{*}{ Similarities } & No Diagnosis & 131 & $11.66^{\mathrm{a}, \mathrm{b}}$ & 0.24 & 2.80 & \\
\hline & ADHD-C & 363 & $12.24^{\mathrm{b}}$ & 0.16 & 3.00 & \\
\hline & ADHD-I & 283 & $12.58^{\mathrm{b}}$ & 0.17 & 2.81 & \\
\hline & Anxiety & 60 & $12.00^{\mathrm{b}}$ & 0.37 & 2.85 & \\
\hline & Depression & 40 & $12.03^{\mathrm{b}}$ & 0.57 & 3.62 & \\
\hline & NVLD & 103 & $13.09^{b}$ & 0.32 & 3.20 & \\
\hline & VLD & 141 & $10.37^{\mathrm{a}}$ & 0.23 & 2.70 & $F(6,1114)=$ \\
\hline & Total & 1121 & 12.08 & 0.09 & 3.02 & $12.04, p<.001$ \\
\hline \multirow[t]{8}{*}{ Arithmetic } & No Diagnosis & 131 & $11.60^{\mathrm{a}, \mathrm{b}, \mathrm{c}}$ & 0.24 & 2.71 & \\
\hline & ADHD-C & 363 & $12.08^{\mathrm{a}, \mathrm{b}, \mathrm{c}}$ & 0.14 & 2.58 & \\
\hline & ADHD-I & 283 & $12.52^{\mathrm{b}, \mathrm{c}}$ & 0.16 & 2.61 & \\
\hline & Anxiety & 60 & $11.87^{\mathrm{a}, \mathrm{b}, \mathrm{c}}$ & 0.28 & 2.19 & \\
\hline & Depression & 40 & $12.65^{\mathrm{c}}$ & 0.54 & 3.41 & \\
\hline & NVLD & 103 & $11.07^{\mathrm{a}}$ & 0.27 & 2.73 & \\
\hline & VLD & 141 & $11.26^{\mathrm{a}, \mathrm{b}}$ & 0.24 & 2.79 & $F(6,1114)=$ \\
\hline & Total & 1121 & 11.95 & 0.08 & 2.70 & $6.63, p<.001$ \\
\hline
\end{tabular}


Table 40

WAIS-III/IV Combined Dataset ANOVA Results for WAIS-III/IV FSIQ and Subtests by Primary Diagnosis, continued

\begin{tabular}{|c|c|c|c|c|c|c|}
\hline $\begin{array}{l}\text { FSIQ*/ } \\
\text { Subtest** }\end{array}$ & Diagnosis & $n$ & M & $\mathrm{SE}$ & SD & $\begin{array}{c}\text { Group } \\
\text { Differences }\end{array}$ \\
\hline \multirow[t]{8}{*}{ Digit Span } & No Diagnosis & 131 & $11.08^{\mathrm{a}, \mathrm{b}}$ & 0.26 & 2.94 & \\
\hline & ADHD-C & 363 & $10.88^{\mathrm{a}}$ & 0.15 & 2.85 & \\
\hline & ADHD-I & 283 & $11.30^{\mathrm{a}, \mathrm{b}}$ & 0.17 & 2.89 & \\
\hline & Anxiety & 60 & $11.57^{\mathrm{a}, \mathrm{b}}$ & 0.34 & 2.63 & \\
\hline & Depression & 40 & $12.43^{b}$ & 0.59 & 3.73 & \\
\hline & NVLD & 103 & $10.73^{\mathrm{a}}$ & 0.29 & 2.89 & \\
\hline & VLD & 141 & $10.21^{\mathrm{a}}$ & 0.23 & 2.75 & $F(6,1114)=$ \\
\hline & Total & 1121 & 11.00 & 0.09 & 2.92 & $4.55, p<.001$ \\
\hline \multirow[t]{8}{*}{ Information } & No Diagnosis & 131 & $12.48^{\mathrm{a}, \mathrm{b}}$ & 0.24 & 2.73 & \\
\hline & ADHD-C & 363 & $12.64^{\mathrm{a}, \mathrm{b}}$ & 0.14 & 2.60 & \\
\hline & ADHD-I & 283 & $13.07^{b}$ & 0.16 & 2.61 & \\
\hline & Anxiety & 60 & $12.33^{\mathrm{a}, \mathrm{b}}$ & 0.30 & 2.33 & \\
\hline & Depression & 40 & $13.63^{b}$ & 0.53 & 3.36 & \\
\hline & NVLD & 103 & $13.45^{\mathrm{b}}$ & 0.27 & 2.74 & \\
\hline & VLD & 141 & $11.39^{\mathrm{a}}$ & 0.25 & 2.97 & $F(6,1114)=$ \\
\hline & Total & 1121 & 12.67 & 0.08 & 2.75 & $8.87, p<.001$ \\
\hline \multirow[t]{8}{*}{ Comprehension } & No Diagnosis & 130 & $12.40^{\mathrm{a}, \mathrm{b}}$ & 0.25 & 2.82 & \\
\hline & ADHD-C & 362 & $12.69^{\mathrm{a}, \mathrm{b}}$ & 0.15 & 2.86 & \\
\hline & ADHD-I & 283 & $12.85^{\mathrm{a}, \mathrm{b}}$ & 0.17 & 2.78 & \\
\hline & Anxiety & 60 & $12.70^{\mathrm{a}, \mathrm{b}}$ & 0.31 & 2.37 & \\
\hline & Depression & 40 & $13.33^{b}$ & 0.60 & 3.77 & \\
\hline & NVLD & 102 & $13.16^{\mathrm{a}, \mathrm{b}}$ & 0.30 & 3.01 & \\
\hline & VLD & 141 & $11.72^{\mathrm{a}}$ & 0.24 & 2.90 & $F(6,1111)=$ \\
\hline & Total & 1118 & 12.64 & 0.09 & 2.89 & $3.77, p=.001$ \\
\hline Letter- & No Diagnosis & 131 & $11.66^{\mathrm{a}, \mathrm{b}}$ & 0.27 & 3.07 & \\
\hline Number & ADHD-C & 363 & $11.12^{\mathrm{a}, \mathrm{b}}$ & 0.16 & 2.99 & \\
\hline \multirow[t]{6}{*}{ Sequencing } & ADHD-I & 283 & $11.55^{\mathrm{a}, \mathrm{b}}$ & 0.19 & 3.20 & \\
\hline & Anxiety & 60 & $11.95^{\mathrm{a}, \mathrm{b}}$ & 0.37 & 2.88 & \\
\hline & Depression & 40 & $12.25^{\mathrm{b}}$ & 0.61 & 3.83 & \\
\hline & NVLD & 103 & $10.52^{\mathrm{a}}$ & 0.30 & 3.01 & \\
\hline & VLD & 141 & $10.70^{\mathrm{a}, \mathrm{b}}$ & 0.26 & 3.06 & $F(6,1114)=$ \\
\hline & Total & 1178 & 11.25 & 0.09 & 3.13 & $3.82, p=.001$ \\
\hline
\end{tabular}


Table 40

WAIS-III/IV Combined Dataset ANOVA Results for WAIS-III/IV FSIQ and Subtests by Primary Diagnosis, continued

\begin{tabular}{|c|c|c|c|c|c|c|}
\hline $\begin{array}{l}\text { FSIQ*/ } \\
\text { Subtest** }\end{array}$ & Diagnosis & $n$ & M & $\mathrm{SE}$ & SD & $\begin{array}{c}\text { Group } \\
\text { Differences }\end{array}$ \\
\hline Picture & No Diagnosis & 131 & $10.27^{b}$ & 0.27 & 3.10 & \\
\hline \multirow[t]{7}{*}{ Completion } & ADHD-C & 363 & $10.21^{\mathrm{b}}$ & 0.15 & 2.88 & \\
\hline & ADHD-I & 282 & $10.40^{\mathrm{b}}$ & 0.17 & 2.83 & \\
\hline & Anxiety & 60 & $10.22^{b}$ & 0.39 & 3.00 & \\
\hline & Depression & 40 & $10.75^{\mathrm{b}}$ & 0.51 & 3.25 & \\
\hline & NVLD & 102 & $8.18^{\mathrm{a}}$ & 0.27 & 2.70 & \\
\hline & VLD & 141 & $10.89^{b}$ & 0.29 & 3.45 & $F(6,1112)=$ \\
\hline & Total & 1119 & 10.19 & 0.09 & 3.04 & $9.56, p<.001$ \\
\hline \multirow[t]{8}{*}{ Coding } & No Diagnosis & 131 & $10.82^{\mathrm{b}}$ & 0.24 & 2.73 & \\
\hline & ADHD-C & 363 & $10.50^{\mathrm{a}, \mathrm{b}}$ & 0.14 & 2.75 & \\
\hline & ADHD-I & 283 & $10.54^{\mathrm{a}, \mathrm{b}}$ & 0.17 & 2.85 & \\
\hline & Anxiety & 60 & $11.02^{b}$ & 0.39 & 3.01 & \\
\hline & Depression & 40 & $11.18^{\mathrm{b}}$ & 0.49 & 3.09 & \\
\hline & NVLD & 103 & $9.23^{\mathrm{a}}$ & 0.33 & 3.31 & \\
\hline & VLD & 141 & $10.65^{a, b}$ & 0.24 & 2.88 & $F(6,1114)=$ \\
\hline & Total & 1121 & 10.50 & 0.09 & 2.90 & $4.39, p<.001$ \\
\hline \multirow[t]{8}{*}{ Block Design } & No Diagnosis & 131 & $11.59^{\mathrm{b}}$ & 0.30 & 3.42 & \\
\hline & ADHD-C & 363 & $12.00^{\mathrm{b}}$ & 0.15 & 2.89 & \\
\hline & ADHD-I & 283 & $12.44^{\mathrm{b}}$ & 0.18 & 3.09 & \\
\hline & Anxiety & 60 & $11.97^{\mathrm{b}}$ & 0.36 & 2.81 & \\
\hline & Depression & 40 & $12.65^{\mathrm{b}}$ & 0.56 & 3.53 & \\
\hline & NVLD & 103 & $9.08^{\mathrm{a}}$ & 0.29 & 2.93 & \\
\hline & VLD & 141 & $12.02^{\mathrm{b}}$ & 0.29 & 3.38 & $F(6,1114)=$ \\
\hline & Total & 1121 & 11.82 & 0.10 & 3.22 & $16.31, p<.001$ \\
\hline Matrix & No Diagnosis & 131 & $12.75^{\mathrm{b}}$ & 0.21 & 2.44 & \\
\hline \multirow[t]{7}{*}{ Reasoning } & ADHD-C & 363 & $12.89^{\mathrm{b}}$ & 0.14 & 2.58 & \\
\hline & ADHD-I & 283 & $13.28^{\mathrm{b}}$ & 0.16 & 2.60 & \\
\hline & Anxiety & 60 & $13.18^{\mathrm{b}}$ & 0.29 & 2.27 & \\
\hline & Depression & 39 & $13.54^{\mathrm{b}}$ & 0.41 & 2.58 & \\
\hline & NVLD & 103 & $10.75^{\mathrm{a}}$ & 0.28 & 2.82 & \\
\hline & VLD & 141 & $13.04^{\mathrm{b}}$ & 0.20 & 2.31 & $F(6,1113)=$ \\
\hline & Total & 1120 & 12.83 & 0.08 & 2.63 & $13.89, p<.001$ \\
\hline
\end{tabular}


Table 40

WAIS-III/IV Combined Dataset ANOVA Results for WAIS-III/IV FSIQ and Subtests by Primary Diagnosis, continued

\begin{tabular}{|c|c|c|c|c|c|c|}
\hline $\begin{array}{l}\text { FSIQ*/ } \\
\text { Subtest** }\end{array}$ & Diagnosis & $n$ & M & $\mathrm{SE}$ & SD & $\begin{array}{c}\text { Group } \\
\text { Differences }\end{array}$ \\
\hline Picture & No Diagnosis & 104 & $11.30^{\mathrm{a}}$ & 0.27 & 2.78 & \\
\hline \multirow[t]{7}{*}{ Arrangement } & ADHD-C & 279 & $11.44^{\mathrm{a}}$ & 0.16 & 2.69 & \\
\hline & ADHD-I & 191 & $11.88^{\mathrm{a}}$ & 0.21 & 2.89 & \\
\hline & Anxiety & 53 & $10.89^{\mathrm{a}}$ & 0.43 & 3.09 & \\
\hline & Depression & 35 & $12.11^{\mathrm{a}}$ & 0.51 & 3.02 & \\
\hline & NVLD & 90 & $10.57^{\mathrm{a}}$ & 0.33 & 3.08 & \\
\hline & VLD & 137 & $11.26^{\mathrm{a}}$ & 0.25 & 2.87 & $F(6,882)=$ \\
\hline & Total & 889 & 11.39 & 0.10 & 2.87 & $2.93, p=.008$ \\
\hline \multirow[t]{8}{*}{ Visual Puzzles } & No Diagnosis & 27 & $10.93^{\mathrm{a}}$ & 0.56 & 2.92 & \\
\hline & ADHD-C & 83 & $10.76^{\mathrm{a}}$ & 0.29 & 2.61 & \\
\hline & ADHD-I & 91 & $11.90^{\mathrm{a}}$ & 0.29 & 2.76 & \\
\hline & Anxiety & 7 & $10.86^{\mathrm{a}}$ & 0.88 & 2.34 & \\
\hline & Depression & 4 & $9.50^{\mathrm{a}}$ & 1.04 & 2.08 & \\
\hline & NVLD & 13 & $7.62^{\mathrm{a}}$ & 0.66 & 2.36 & \\
\hline & VLD & 4 & $10.75^{\mathrm{a}}$ & 2.84 & 5.68 & $F(6,222)=$ \\
\hline & Total & 229 & 11.03 & 0.19 & 2.90 & $5.23, p<.001$ \\
\hline \multirow[t]{8}{*}{ Figure Weights } & No Diagnosis & 26 & 12.54 & 0.56 & 2.85 & \\
\hline & ADHD-C & 83 & 12.42 & 0.31 & 2.83 & \\
\hline & ADHD-I & 91 & 13.02 & 0.29 & 2.77 & \\
\hline & Anxiety & 7 & 12.86 & 1.08 & 2.85 & \\
\hline & Depression & 4 & 11.00 & 0.58 & 1.16 & \\
\hline & NVLD & 13 & 10.92 & 0.98 & 3.52 & \\
\hline & VLD & 4 & 11.00 & 2.74 & 5.48 & $F(6,221)=$ \\
\hline & Total & 228 & 12.55 & 0.19 & 2.91 & $1.52, p=.174$ \\
\hline \multirow[t]{8}{*}{ Symbol Search } & No Diagnosis & 131 & $11.08^{\mathrm{a}, \mathrm{b}}$ & 0.23 & 2.60 & \\
\hline & ADHD-C & 363 & $11.16^{\mathrm{a}, \mathrm{b}}$ & 0.16 & 2.98 & \\
\hline & ADHD-I & 283 & $10.87^{\mathrm{a}, \mathrm{b}}$ & 0.17 & 2.88 & \\
\hline & Anxiety & 60 & $11.48^{\mathrm{a}, \mathrm{b}}$ & 0.42 & 3.26 & \\
\hline & Depression & 39 & $11.90^{\mathrm{b}}$ & 0.50 & 3.13 & \\
\hline & NVLD & 103 & $9.97^{\mathrm{a}}$ & 0.31 & 3.13 & \\
\hline & VLD & 141 & $11.23^{\mathrm{a}, \mathrm{b}}$ & 0.28 & 3.30 & $F(6,1113)=$ \\
\hline & Total & 1120 & 11.02 & 0.09 & 3.01 & $3.28, p=.003$ \\
\hline
\end{tabular}


Table 40

WAIS-III/IV Combined Dataset ANOVA Results for WAIS-III/IV FSIQ and Subtests by Primary Diagnosis, continued

\begin{tabular}{llrrrrc}
\hline $\begin{array}{l}\text { FSIQ*/ } \\
\text { Subtest** }\end{array}$ & Diagnosis & $n$ & M & SE & SD & $\begin{array}{c}\text { Group } \\
\text { Differences }\end{array}$ \\
\hline Cancellation & No Diagnosis & 6 & 10.50 & 1.12 & 2.74 & \\
& ADHD-C & 27 & 10.22 & 0.64 & 3.34 & \\
& ADHD-I & 24 & 9.38 & 0.60 & 2.95 & \\
& Anxiety & 1 & 8.00 & - & - & \\
& Depression & 0 & - & - & - & \\
& NVLD & 3 & 8.67 & 0.88 & 1.53 & \\
& VLD & 1 & 8.00 & - & - & $F(6,56)=$ \\
& Total & 62 & 9.77 & 0.38 & 3.02 & $0.47, p=.796$ \\
\hline
\end{tabular}

Note. WAIS-III/IV = Wechsler Adult Intelligence Scale - Third/Fourth Editions. FSIQ $=$ Full-Scale Intelligence Quotient. $\mathrm{M}=$ Mean. $\mathrm{SE}=$ Standard Error of the Mean. SD $=$ Standard Deviation. *WAIS-III/IV FSIQs have a Standard Score $\mathrm{M}=$ $100, \mathrm{SD}=15 .{ }^{*}$ Subtests have an Index Score $\mathrm{M}=10, \mathrm{SD}=3$. ADHD-C $=$ Attention Deficit Hyperactivity Disorder - Combined Type. ADHD-I = Attention Deficit Hyperactivity Disorder, Predominantly Inattentive Type. NVLD = Nonverbal Learning Disability. VLD = Verbal Learning Disability. Means sharing the same superscript are not significantly different from each other (Scheffé's, $p<0.05$ ).

Table 41

WAIS-III/IV Combined Dataset ANOVA Results for the WJ III Cognitive GIA and Subtests by Primary Diagnosis

\begin{tabular}{llrcccc}
\hline GIA/ Subtest* & \multicolumn{1}{c}{ Diagnosis } & $n$ & $\mathrm{M}$ & $\mathrm{SE}$ & $\mathrm{SD}$ & Group Differences \\
\hline GIA & No Diagnosis & 114 & $103.15^{\mathrm{a}, \mathrm{b}}$ & 1.11 & 11.82 & \\
& ADHD-C & 363 & $103.13^{\mathrm{a}, \mathrm{b}}$ & 0.63 & 12.02 & \\
& ADHD-I & 282 & $106.03^{\mathrm{b}}$ & 0.65 & 10.91 & \\
& Anxiety & 60 & $103.65^{\mathrm{a}, \mathrm{b}}$ & 1.35 & 10.47 & \\
& Depression & 39 & $108.00^{\mathrm{b}}$ & 2.26 & 14.09 & \\
NVLD & 105 & $99.14^{\mathrm{a}}$ & 1.22 & 12.52 & \\
& VLD & 142 & $98.99^{\mathrm{a}}$ & 1.11 & 13.24 & $F(6,1098)=$ \\
& Total & 1105 & 103.16 & 0.37 & 12.19 & $8.66, p<.001$
\end{tabular}


Table 41

WAIS-III/IV Combined Dataset ANOVA Results for the WJ III Cognitive GIA and Subtests by Primary Diagnosis, continued

\begin{tabular}{|c|c|c|c|c|c|c|}
\hline GIA/ Subtest* & Diagnosis & $n$ & $\mathrm{M}$ & $\mathrm{SE}$ & SD & Group Differences \\
\hline Verbal & No Diagnosis & 114 & $101.95^{\mathrm{a}, \mathrm{b}}$ & 1.09 & 11.64 & \\
\hline \multirow[t]{7}{*}{ Comprehension } & ADHD-C & 323 & $103.85^{\mathrm{b}}$ & 0.64 & 11.44 & \\
\hline & ADHD-I & 278 & $106.32^{b}$ & 0.69 & 11.46 & \\
\hline & Anxiety & 53 & $101.96^{\mathrm{a}, \mathrm{b}}$ & 1.37 & 9.93 & \\
\hline & Depression & 38 & $106.97^{\mathrm{b}}$ & 2.21 & 13.64 & \\
\hline & NVLD & 86 & $102.71^{b}$ & 1.35 & 12.51 & \\
\hline & VLD & 108 & $95.92^{\mathrm{a}}$ & 1.34 & 13.93 & $F(6,993)=$ \\
\hline & Total & 1000 & 103.38 & 0.39 & 12.22 & $11.08, p<.001$ \\
\hline Visual- & No Diagnosis & 115 & $96.92^{\mathrm{a}, \mathrm{b}}$ & 1.44 & 15.39 & \\
\hline Auditory & ADHD-C & 362 & $98.40^{\mathrm{a}, \mathrm{b}}$ & 1.00 & 19.06 & \\
\hline \multirow{6}{*}{ Learning } & ADHD-I & 283 & $101.61^{\mathrm{a}, \mathrm{b}}$ & 1.04 & 17.51 & \\
\hline & Anxiety & 60 & $99.23^{\mathrm{a}, \mathrm{b}}$ & 1.88 & 14.53 & \\
\hline & Depression & 39 & $105.21^{\mathrm{b}}$ & 3.54 & 22.08 & \\
\hline & NVLD & 105 & $94.59^{\mathrm{a}}$ & 2.05 & 21.00 & \\
\hline & VLD & 140 & $99.04^{\mathrm{a}, \mathrm{b}}$ & 1.53 & 18.12 & $F(6,1097)=$ \\
\hline & Total & 1104 & 99.07 & 0.55 & 18.39 & $3.04, p<.006$ \\
\hline Spatial & No Diagnosis & 114 & $104.95^{\mathrm{b}}$ & 1.13 & 12.03 & \\
\hline \multirow[t]{7}{*}{ Relations } & ADHD-C & 323 & $104.70^{\mathrm{b}}$ & 0.64 & 11.47 & \\
\hline & ADHD-I & 278 & $107.56^{\mathrm{b}}$ & 0.59 & 9.81 & \\
\hline & Anxiety & 53 & $106.17^{\mathrm{b}}$ & 1.51 & 11.00 & \\
\hline & Depression & 38 & $109.03^{b}$ & 1.33 & 8.19 & \\
\hline & NVLD & 84 & $97.04^{\mathrm{a}}$ & 1.17 & 10.76 & \\
\hline & VLD & 107 & $104.81^{\mathrm{b}}$ & 1.11 & 11.46 & $F(6,990)=$ \\
\hline & Total & 997 & 105.13 & 0.36 & 11.23 & $11.01, p<.001$ \\
\hline Sound & No Diagnosis & 115 & $105.05^{\mathrm{b}}$ & 1.10 & 11.83 & \\
\hline \multirow[t]{7}{*}{ Blending } & ADHD-C & 361 & $104.94^{\mathrm{b}}$ & 0.65 & 12.31 & \\
\hline & ADHD-I & 282 & $106.71^{b}$ & 0.70 & 11.80 & \\
\hline & Anxiety & 60 & $106.00^{\mathrm{b}}$ & 1.68 & 13.02 & \\
\hline & Depression & 38 & $107.18^{\mathrm{b}}$ & 2.16 & 13.30 & \\
\hline & NVLD & 106 & $104.00^{\mathrm{b}}$ & 1.22 & 12.52 & \\
\hline & VLD & 141 & $96.94^{\mathrm{a}}$ & 1.16 & 13.71 & $F(6,1096)=$ \\
\hline & Total & 1103 & 104.42 & 0.38 & 12.74 & $10.78, p<.001$ \\
\hline
\end{tabular}


Table 41

WAIS-III/IV Combined Dataset ANOVA Results for the WJ III Cognitive GIA and Subtests by Primary Diagnosis, continued

\begin{tabular}{|c|c|c|c|c|c|c|}
\hline GIA/ Subtest* & Diagnosis & $n$ & M & SE & SD & Group Differences \\
\hline Concept & No Diagnosis & 114 & $106.48^{\mathrm{a}, \mathrm{b}}$ & 1.16 & 12.38 & \\
\hline \multirow[t]{7}{*}{ Formation } & ADHD-C & 364 & $107.98^{\mathrm{a}, \mathrm{b}}$ & 0.60 & 11.37 & \\
\hline & ADHD-I & 282 & $109.84^{\mathrm{b}}$ & 0.62 & 10.35 & \\
\hline & Anxiety & 60 & $107.28^{\mathrm{a}, \mathrm{b}}$ & 1.54 & 11.90 & \\
\hline & Depression & 39 & $106.44^{\mathrm{a}, \mathrm{b}}$ & 2.07 & 12.91 & \\
\hline & NVLD & 106 & $103.11^{\mathrm{a}}$ & 1.17 & 12.08 & \\
\hline & VLD & 141 & $105.57^{\mathrm{a}, \mathrm{b}}$ & 1.31 & 15.53 & $F(6,1099)=$ \\
\hline & Total & 1106 & 107.43 & 0.37 & 12.12 & $5.04, p<.001$ \\
\hline Visual & No Diagnosis & 115 & $103.34^{\mathrm{b}}$ & 1.43 & 15.35 & \\
\hline \multirow[t]{7}{*}{ Matching } & ADHD-C & 363 & $101.12^{\mathrm{a}, \mathrm{b}}$ & 0.81 & 15.47 & \\
\hline & ADHD-I & 283 & $101.40^{\mathrm{a}, \mathrm{b}}$ & 0.87 & 14.58 & \\
\hline & Anxiety & 60 & $103.83^{\mathrm{b}}$ & 1.78 & 13.82 & \\
\hline & Depression & 39 & $105.59^{\mathrm{b}}$ & 2.81 & 17.57 & \\
\hline & NVLD & 106 & $95.06^{\mathrm{a}}$ & 1.52 & 15.69 & \\
\hline & VLD & 142 & $101.39^{\mathrm{a}, \mathrm{b}}$ & 1.48 & 17.61 & $F(6,1101)=$ \\
\hline & Total & 1108 & 101.18 & 0.47 & 15.66 & $3.94, p=.001$ \\
\hline Numbers & No Diagnosis & 110 & $106.32^{\mathrm{b}}$ & 1.32 & 13.86 & \\
\hline \multirow[t]{7}{*}{ Reversed } & ADHD-C & 296 & $102.29^{\mathrm{a}, \mathrm{b}}$ & 0.83 & 14.20 & \\
\hline & ADHD-I & 278 & $106.27^{\mathrm{b}}$ & 0.86 & 14.34 & \\
\hline & Anxiety & 47 & $104.83^{\mathrm{a}, \mathrm{b}}$ & 1.81 & 12.44 & \\
\hline & Depression & 36 & $108.00^{\mathrm{b}}$ & 2.59 & 15.55 & \\
\hline & NVLD & 76 & $100.33^{\mathrm{a}, \mathrm{b}}$ & 1.87 & 16.27 & \\
\hline & VLD & 91 & $97.45^{\mathrm{a}}$ & 1.64 & 15.64 & \\
\hline & Total & 934 & 103.67 & 0.48 & 14.76 & $F(6,927)=$ \\
\hline Incomplete & No Diagnosis & 109 & $108.38^{\mathrm{a}}$ & 1.17 & 12.20 & $6.60, p<.001$ \\
\hline \multirow{7}{*}{ Words } & ADHD-C & 361 & $105.87^{\mathrm{a}}$ & 0.76 & 14.50 & \\
\hline & ADHD-I & 281 & $108.28^{\mathrm{a}}$ & 0.80 & 13.39 & \\
\hline & Anxiety & 59 & $105.59^{\mathrm{a}}$ & 1.75 & 13.44 & \\
\hline & Depression & 38 & $106.58^{\mathrm{a}}$ & 3.50 & 21.57 & \\
\hline & NVLD & 105 & $105.31^{\mathrm{a}}$ & 1.50 & 15.40 & \\
\hline & VLD & 141 & $101.14^{\mathrm{a}}$ & 1.30 & 15.41 & $F(6,1087)=$ \\
\hline & Total & 1094 & 106.09 & 0.44 & 14.60 & $4.36, \quad p<.001$ \\
\hline
\end{tabular}


Table 41

WAIS-III/IV Combined Dataset ANOVA Results for the WJ III Cognitive GIA and Subtests by Primary Diagnosis, continued

\begin{tabular}{|c|c|c|c|c|c|c|}
\hline GIA/ Subtest* & Diagnosis & $n$ & M & $\mathrm{SE}$ & $\mathrm{SD}$ & Group Differences \\
\hline Retrieval & No Diagnosis & 114 & 97.86 & 1.02 & 10.92 & \\
\hline \multirow[t]{7}{*}{ Fluency } & ADHD-C & 324 & 98.95 & 0.62 & 11.12 & \\
\hline & ADHD-I & 278 & 96.49 & 0.64 & 10.72 & \\
\hline & Anxiety & 52 & 97.40 & 1.40 & 10.10 & \\
\hline & Depression & 38 & 98.37 & 2.66 & 16.38 & \\
\hline & NVLD & 84 & 98.07 & 1.18 & 10.79 & \\
\hline & VLD & 107 & 98.06 & 1.05 & 10.81 & $F(6,990)=$ \\
\hline & Total & 997 & 97.87 & 0.35 & 11.13 & $1.26, p=.272$ \\
\hline Picture & No Diagnosis & 114 & $102.54^{\mathrm{a}}$ & 0.99 & 10.56 & \\
\hline \multirow[t]{7}{*}{ Recognition } & ADHD-C & 361 & $101.96^{\mathrm{a}}$ & 0.62 & 11.82 & \\
\hline & ADHD-I & 282 & $102.12^{\mathrm{a}}$ & 0.69 & 11.58 & \\
\hline & Anxiety & 59 & $102.88^{\mathrm{a}}$ & 1.64 & 12.59 & \\
\hline & Depression & 39 & $102.77^{\mathrm{a}}$ & 2.18 & 13.59 & \\
\hline & NVLD & 106 & $98.08^{\mathrm{a}}$ & 1.39 & 14.26 & \\
\hline & VLD & 140 & $102.05^{\mathrm{a}}$ & 0.97 & 11.42 & $F(6,1094)=$ \\
\hline & Total & 1101 & 101.78 & 0.36 & 11.98 & $1.97, p=.068$ \\
\hline Analysis- & No Diagnosis & 114 & $107.94^{\mathrm{a}, \mathrm{b}}$ & 1.06 & 11.28 & \\
\hline \multirow[t]{7}{*}{ Synthesis } & ADHD-C & 361 & $109.44^{\mathrm{a}, \mathrm{b}, \mathrm{c}}$ & 0.69 & 13.06 & \\
\hline & ADHD-I & 283 & $111.36^{\mathrm{b}, \mathrm{c}}$ & 0.74 & 12.47 & \\
\hline & Anxiety & 60 & $112.52^{\mathrm{b}, \mathrm{c}}$ & 1.77 & 13.68 & \\
\hline & Depression & 39 & $114.97^{\mathrm{c}}$ & 2.40 & 14.99 & \\
\hline & NVLD & 105 & $102.94^{\mathrm{a}}$ & 1.41 & 14.42 & \\
\hline & VLD & 142 & $107.38^{\mathrm{a}, \mathrm{b}}$ & 1.31 & 15.58 & $F(6,1097)=$ \\
\hline & Total & 1104 & 109.26 & 0.41 & 13.57 & $7.55, p<.001$ \\
\hline \multirow[t]{8}{*}{ Decision Speed } & No Diagnosis & 114 & $104.19^{b}$ & 1.39 & 14.89 & \\
\hline & ADHD-C & 324 & $103.25^{\mathrm{a}, \mathrm{b}}$ & 0.90 & 16.18 & \\
\hline & ADHD-I & 278 & $99.83^{\mathrm{a}, \mathrm{b}}$ & 0.93 & 15.49 & \\
\hline & Anxiety & 53 & $105.19^{b}$ & 2.16 & 15.69 & \\
\hline & Depression & 38 & $105.24^{b}$ & 3.17 & 19.53 & \\
\hline & NVLD & 84 & $95.45^{\mathrm{a}}$ & 1.95 & 17.86 & \\
\hline & VLD & 107 & $102.23^{\mathrm{a}, \mathrm{b}}$ & 1.51 & 15.64 & $F(6,991)=$ \\
\hline & Total & 998 & 101.82 & 0.51 & 16.22 & $4.45, p<.001$ \\
\hline
\end{tabular}


Table 41

WAIS-III/IV Combined Dataset ANOVA Results for the WJ III Cognitive GIA and Subtests by Primary Diagnosis, continued

\begin{tabular}{|c|c|c|c|c|c|c|}
\hline GIA/ Subtest* & Diagnosis & $n$ & M & $\mathrm{SE}$ & SD & Group Differences \\
\hline Memory for & No Diagnosis & 114 & $104.27^{\mathrm{a}, \mathrm{b}}$ & 1.34 & 14.33 & \\
\hline \multirow[t]{7}{*}{ Words } & ADHD-C & 362 & $101.74^{\mathrm{a}, \mathrm{b}}$ & 0.72 & 13.74 & \\
\hline & ADHD-I & 281 & $103.59^{\mathrm{a}, \mathrm{b}}$ & 0.79 & 13.22 & \\
\hline & Anxiety & 60 & $104.03^{\mathrm{a}, \mathrm{b}}$ & 1.52 & 11.79 & \\
\hline & Depression & 39 & $106.05^{\mathrm{b}}$ & 2.41 & 15.05 & \\
\hline & NVLD & 105 & $103.15^{\mathrm{a}, \mathrm{b}}$ & 1.33 & 13.65 & \\
\hline & VLD & 141 & $97.37^{\mathrm{a}}$ & 1.33 & 14.19 & $F(6,1095)=$ \\
\hline & Total & 1102 & 103.07 & 0.82 & 27.18 & $4.70, p<.001$ \\
\hline Rapid & No Diagnosis & 114 & 97.15 & 1.40 & 14.93 & \\
\hline Picture & ADHD-C & 322 & 96.84 & 0.94 & 16.86 & \\
\hline \multirow[t]{6}{*}{ Naming } & ADHD-I & 279 & 95.01 & 0.99 & 16.58 & \\
\hline & Anxiety & 53 & 98.36 & 2.30 & 16.76 & \\
\hline & Depression & 37 & 100.86 & 2.51 & 15.28 & \\
\hline & NVLD & 84 & 97.99 & 1.87 & 17.16 & \\
\hline & VLD & 105 & 95.49 & 1.55 & 15.85 & $F(6,987)=$ \\
\hline & Total & 994 & 96.55 & 0.52 & 16.44 & $1.16, p=.324$ \\
\hline \multirow[t]{8}{*}{ Planning } & No Diagnosis & 113 & $112.76^{\mathrm{a}, \mathrm{b}}$ & 2.23 & 23.73 & \\
\hline & ADHD-C & 322 & $110.79^{a, b}$ & 1.25 & 22.37 & \\
\hline & ADHD-I & 276 & $116.95^{\mathrm{a}, \mathrm{b}}$ & 1.68 & 27.85 & \\
\hline & Anxiety & 53 & $115.11^{\mathrm{a}, \mathrm{b}}$ & 3.08 & 22.44 & \\
\hline & Depression & 38 & $120.68^{b}$ & 4.86 & 29.93 & \\
\hline & NVLD & 83 & $104.05^{\mathrm{a}}$ & 1.61 & 14.65 & \\
\hline & VLD & 107 & $110.25^{\mathrm{a}, \mathrm{b}}$ & 1.92 & 19.91 & $F(6,985)=$ \\
\hline & Total & 992 & 112.71 & 0.76 & 24.02 & $4.65, p<.001$ \\
\hline Pair & No Diagnosis & 113 & $101.59^{b}$ & 1.32 & 14.07 & \\
\hline \multirow[t]{7}{*}{ Cancellation } & ADHD-C & 324 & $99.82^{\mathrm{a}, \mathrm{b}}$ & 0.87 & 15.74 & \\
\hline & ADHD-I & 278 & $98.50^{\mathrm{a}, \mathrm{b}}$ & 0.83 & 13.81 & \\
\hline & Anxiety & 53 & $103.19^{b}$ & 1.73 & 12.56 & \\
\hline & Depression & 38 & $103.00^{\mathrm{b}}$ & 2.69 & 16.56 & \\
\hline & NVLD & 83 & $93.54^{\mathrm{a}}$ & 1.42 & 12.91 & \\
\hline & VLD & 109 & $101.18^{\mathrm{a}, \mathrm{b}}$ & 1.51 & 15.81 & $F(6,991)=$ \\
\hline & Total & 998 & 99.58 & 0.47 & 14.83 & $4.05, p=.001$ \\
\hline
\end{tabular}


Table 41

WAIS-III/IV Combined Dataset ANOVA Results for the WJ III Cognitive GIA and Subtests by Primary Diagnosis, continued

Note. WAIS-III/IV = Wechsler Adult Intelligence Scale - Third/Fourth Editions. WJ III $=$ Woodcock-Johnson Tests of Cognitive Abilities - Third Edition. GIA $=$ General Intellectual Ability. $\mathrm{M}=$ Mean. $\mathrm{SE}=$ Standard Error of the Mean. $\mathrm{SD}=$ Standard Deviation. *WJ III has a Standard Score $\mathrm{M}=100, \mathrm{SD}=15$. ADHD-C $=$ Attention Deficit Hyperactivity Disorder - Combined Type. ADHD-I = Attention Deficit Hyperactivity Disorder, Predominantly Inattentive Type. NVLD = Nonverbal Learning Disability.VLD = Verbal Learning Disability. Means sharing the same superscript are not significantly different from each other (Scheffé's, $p<0.05$ ).

Table 42

WAIS-III/IV Combined Dataset ANOVA Results for the WJ III Achievement Subtests by Primary Diagnosis

\begin{tabular}{llrcrrr}
\hline Subtest* & Diagnosis & $n$ & $\mathrm{M}$ & $\mathrm{SE}$ & $\mathrm{SD}$ & Group Differences \\
\hline Letter-Word & No Diagnosis & 115 & $103.44^{\mathrm{b}}$ & 0.80 & 8.62 & \\
Identification & ADHD-C & 366 & $104.32^{\mathrm{b}}$ & 0.55 & 10.45 & \\
& ADHD-I & 282 & $106.43^{\mathrm{b}}$ & 0.55 & 9.23 & \\
& Anxiety & 59 & $103.64^{\mathrm{b}}$ & 1.11 & 8.51 & \\
& Depression & 39 & $105.90^{\mathrm{b}}$ & 1.48 & 9.27 & \\
& NVLD & 107 & $106.91^{\mathrm{b}}$ & 1.11 & 11.44 & \\
& VLD & 145 & $96.29^{\mathrm{a}}$ & 0.99 & 11.95 & $F(6,1106)=$ \\
Reading & Total & 1113 & 103.98 & 0.32 & 10.63 & $18.43, p<.001$ \\
& No Diagnosis & 115 & $103.90^{\mathrm{b}}$ & 1.21 & 12.98 & \\
& ADHD-C & 324 & $101.81^{\mathrm{b}}$ & 0.82 & 14.72 & \\
& ADHD-I & 278 & $102.68^{\mathrm{b}}$ & 0.84 & 13.94 & \\
& Anxiety & 53 & $103.23^{\mathrm{b}}$ & 1.91 & 13.88 & \\
& Depression & 38 & $107.84^{\mathrm{b}}$ & 2.79 & 17.20 & \\
& NVLD & 85 & $103.06^{\mathrm{b}}$ & 1.67 & 15.41 & \\
& VLD & 108 & $90.83^{\mathrm{a}}$ & 1.19 & 12.37 & $F(6,994)=$ \\
& Total & 1001 & $101.52^{\mathrm{b}}$ & 0.46 & 14.68 & $12.63, p<.001$ \\
& No Diagnosis & 115 & $109.03^{\mathrm{a}, \mathrm{b}}$ & 1.35 & 14.50 & \\
& ADHD-C & 361 & $111.34^{\mathrm{b}, \mathrm{c}}$ & 0.77 & 14.66 & \\
& ADHD-I & 283 & $114.33^{\mathrm{b}, \mathrm{c}}$ & 0.81 & 13.70 & \\
& Anxiety & 60 & $113.15^{\mathrm{b}, \mathrm{c}}$ & 1.72 & 13.35 & \\
& Depression & 40 & $118.75^{\mathrm{c}}$ & 2.62 & 16.54 & \\
& NVLD & 102 & $102.25^{\mathrm{a}}$ & 1.57 & 15.84 & \\
& VLD & 143 & $106.73^{\mathrm{a}, \mathrm{b}}$ & 1.49 & 17.78 & $F(6,1097)=$ \\
& Total & 1104 & 110.79 & 0.46 & 15.42 & $12.40, p<.001$ \\
& & & & &
\end{tabular}


Table 42

WAIS-III/IV Combined Dataset ANOVA Results for the WJ III Achievement Subtests by Primary Diagnosis, continued

\begin{tabular}{|c|c|c|c|c|c|c|}
\hline Subtest* & Diagnosis & $n$ & $\mathrm{M}$ & $\mathrm{SE}$ & SD & $\begin{array}{c}\text { Group } \\
\text { Differences }\end{array}$ \\
\hline \multirow[t]{8}{*}{ Math Fluency } & No Diagnosis & 115 & $100.80^{\mathrm{a}, \mathrm{b}, \mathrm{c}}$ & 1.25 & 13.37 & \\
\hline & ADHD-C & 324 & $100.73^{\mathrm{a}, \mathrm{b}, \mathrm{c}}$ & 0.80 & 14.36 & \\
\hline & ADHD-I & 278 & $101.18^{\mathrm{a}, \mathrm{b}, \mathrm{c}}$ & 0.82 & 13.73 & \\
\hline & Anxiety & 52 & $105.38^{\mathrm{c}}$ & 1.80 & 13.01 & \\
\hline & Depression & 39 & $104.51^{b, c}$ & 2.95 & 18.44 & \\
\hline & NVLD & 85 & $97.00^{\mathrm{a}, \mathrm{b}}$ & 1.37 & 12.66 & \\
\hline & VLD & 107 & $95.89^{\mathrm{a}}$ & 1.28 & 13.24 & $F(6,993)=$ \\
\hline & Total & 1000 & 100.42 & 0.45 & 14.08 & $4.58, p<.001$ \\
\hline \multirow[t]{8}{*}{ Spelling } & No Diagnosis & 114 & $106.98^{b}$ & 0.81 & 8.65 & \\
\hline & ADHD-C & 324 & $106.89^{b}$ & 0.52 & 9.34 & \\
\hline & ADHD-I & 277 & $109.64^{\mathrm{b}}$ & 0.52 & 8.72 & \\
\hline & Anxiety & 53 & $107.96^{\mathrm{b}}$ & 1.32 & 9.59 & \\
\hline & Depression & 38 & $108.53^{b}$ & 1.87 & 11.50 & \\
\hline & NVLD & 87 & $107.13^{b}$ & 1.19 & 11.11 & \\
\hline & VLD & 109 & $96.78^{\mathrm{a}}$ & 0.96 & 10.07 & $F(6,995)=$ \\
\hline & Total & 1002 & 106.70 & 0.32 & 10.10 & $24.96, p<.001$ \\
\hline Writing & No Diagnosis & 115 & $109.05^{\mathrm{b}}$ & 1.35 & 14.43 & \\
\hline \multirow[t]{7}{*}{ Fluency } & ADHD-C & 364 & $109.46^{\mathrm{b}}$ & 0.74 & 14.15 & \\
\hline & ADHD-I & 281 & $108.01^{\mathrm{a}, \mathrm{b}}$ & 0.77 & 12.82 & \\
\hline & Anxiety & 57 & $107.93^{\mathrm{a}, \mathrm{b}}$ & 1.95 & 14.72 & \\
\hline & Depression & 39 & $109.38^{\mathrm{b}}$ & 1.75 & 10.91 & \\
\hline & NVLD & 105 & $106.75^{\mathrm{a}, \mathrm{b}}$ & 1.66 & 17.01 & \\
\hline & VLD & 137 & $100.94^{\mathrm{a}}$ & 1.23 & 14.39 & $F(6,1091)=$ \\
\hline & Total & 1098 & 107.64 & 0.43 & 14.33 & $6.55, p<.001$ \\
\hline Passage & No Diagnosis & 115 & $104.37^{b}$ & 0.93 & 10.00 & \\
\hline \multirow[t]{7}{*}{ Comprehension } & ADHD-C & 364 & $105.13^{b}$ & 0.51 & 9.73 & \\
\hline & ADHD-I & 282 & $107.76^{\mathrm{b}}$ & 0.63 & 10.55 & \\
\hline & Anxiety & 58 & $105.41^{\mathrm{b}}$ & 1.39 & 10.60 & \\
\hline & Depression & 39 & $108.31^{b}$ & 1.78 & 11.12 & \\
\hline & NVLD & 107 & $104.78^{b}$ & 1.01 & 10.47 & \\
\hline & VLD & 146 & $97.52^{\mathrm{a}}$ & 0.99 & 11.90 & $F(6,1104)=$ \\
\hline & Total & 1111 & 104.81 & 0.33 & 10.87 & $16.49, p<.001$ \\
\hline
\end{tabular}


Table 42

WAIS-III/IV Combined Dataset ANOVA Results for the WJ III Achievement Subtests by Primary Diagnosis, continued

\begin{tabular}{|c|c|c|c|c|c|c|}
\hline Subtest* & Diagnosis & $n$ & M & $\mathrm{SE}$ & $\mathrm{SD}$ & $\begin{array}{c}\text { Group } \\
\text { Differences }\end{array}$ \\
\hline Applied & No Diagnosis & 114 & $105.52^{\mathrm{a}, \mathrm{b}, \mathrm{c}}$ & 1.20 & 12.83 & \\
\hline \multirow[t]{7}{*}{ Problems } & ADHD-C & 364 & $107.58^{\mathrm{b}, \mathrm{c}}$ & 0.68 & 12.89 & \\
\hline & ADHD-I & 283 & $110.47^{\mathrm{c}}$ & 0.73 & 12.29 & \\
\hline & Anxiety & 56 & $107.98^{\mathrm{b}, \mathrm{c}}$ & 1.53 & 11.42 & \\
\hline & Depression & 39 & $109.15^{\mathrm{b}, \mathrm{c}}$ & 2.11 & 13.19 & \\
\hline & NVLD & 107 & $100.58^{\mathrm{a}}$ & 1.29 & 13.36 & \\
\hline & VLD & 145 & $102.70^{\mathrm{a}, \mathrm{b}}$ & 1.10 & 13.25 & $F(6,1101)=$ \\
\hline & Total & 1108 & 106.87 & 0.39 & 13.12 & $11.33,<.001$ \\
\hline Writing & No Diagnosis & 114 & $105.98^{\mathrm{a}, \mathrm{b}}$ & 1.11 & 11.81 & \\
\hline \multirow[t]{7}{*}{ Samples } & ADHD-C & 366 & $108.49^{\mathrm{a}, \mathrm{b}}$ & 0.82 & 15.62 & \\
\hline & ADHD-I & 281 & $112.14^{\mathrm{b}}$ & 0.84 & 14.07 & \\
\hline & Anxiety & 59 & $113.00^{\mathrm{b}}$ & 2.40 & 18.40 & \\
\hline & Depression & 39 & $110.85^{\mathrm{b}}$ & 2.93 & 18.32 & \\
\hline & NVLD & 107 & $109.34^{\mathrm{a}, \mathrm{b}}$ & 1.83 & 18.90 & \\
\hline & VLD & 146 & $101.69^{\mathrm{a}}$ & 1.46 & 17.58 & $F(6,1105)=$ \\
\hline & Total & 1112 & 108.67 & 0.48 & 16.10 & $8.47, p<.001$ \\
\hline \multirow[t]{8}{*}{ Word Attack } & No Diagnosis & 111 & $102.54^{\mathrm{b}}$ & 1.11 & 9.48 & \\
\hline & ADHD-C & 360 & $102.94^{\mathrm{b}}$ & 0.58 & 10.95 & \\
\hline & ADHD-I & 279 & $104.47^{\mathrm{b}}$ & 0.56 & 9.40 & \\
\hline & Anxiety & 56 & $101.36^{\mathrm{b}}$ & 1.26 & 9.46 & \\
\hline & Depression & 39 & $102.44^{\mathrm{b}}$ & 1.98 & 12.38 & \\
\hline & NVLD & 107 & $101.92^{b}$ & 1.12 & 11.58 & \\
\hline & VLD & 134 & $94.60^{\mathrm{a}}$ & 1.10 & 12.73 & $F(6,1079)=$ \\
\hline & Total & 1086 & 102.06 & 0.34 & 11.09 & $13.65, p<.001$ \\
\hline
\end{tabular}

Note. $\mathrm{M}=$ Mean. $\mathrm{SE}=$ Standard Error of the Mean. $\mathrm{SD}=$ Standard Deviation. ${ }^{*} \mathrm{WJ}$ III has a Standard Score $\mathrm{M}=100, \mathrm{SD}=15$. ADHD-C $=$ Attention Deficit Hyperactivity Disorder - Combined Type. ADHD-I = Attention Deficit Hyperactivity Disorder, Predominantly Inattentive Type. NVLD = Nonverbal Learning Disability. VLD = Verbal Learning Disability. Means sharing the same superscript are not significantly different from each other (Scheffé's, $p<0.05$ ).

As expected, the CAARS self- and other-rating scales significantly differentiated ADHD diagnoses. Since the CAARS is used to help diagnose ADHD, it would have been surprising if these findings were otherwise. Table 43 illustrates the results of the 
analyses of all CAARS subscales by diagnosis. The ADHD-C and ADHD-I groups obtained higher scores than the other diagnosis groups on all self- and other-rated subtests except Problems with Self Concept, for which all diagnoses were similar to at least two other diagnoses on both subscales. Otherwise, the ADHD-C group consistently scored in the highest group on all self- and other-rated subscales, and, also as expected, it did not significantly differ from the ADHD-I group on Inattention/Memory Problems, DSM-IV Inattentive Symptoms, or the ADHD Index. On both the self- and other-rated scales, the ADHD-C group singularly scored higher than any other group on Hyperactivity/ Restlessness, DSM-IV Hyperactive/ Impulsive Symptoms, and DSM-IV ADHD Symptoms Total. On the Impulsivity/Emotional Lability subscale, The ADHD-C group scored highest only on the self-rated subscale; the high group on the other-rated subscale was shared by the ADHD-I and NVLD diagnostic groups. One noteworthy finding concerning the VLD diagnostic group is that its scores very nearly matched the No Diagnosis group with scores in the lowest group on every CAARS subscale. The Anxiety group also shared the VLD and No Diagnosis group as the lowest-rated group for ADHD symptoms on most subscales. The Depression group varied in its responses, typically scoring below ADHD-C but above VLD and No Diagnosis in most instances. Interestingly, the Depression group also obtained the highest mean scores for both scales' Problems with Self Concept subscale, although its mean scores significantly differed from the mean scores of only the No Diagnosis and VLD groups on the self-rated scale, and from the mean score of only the No Diagnosis group on the other-rated scale. 
Table 43

WAIS-III/IV Combined Dataset ANOVA Results for the CAARS Self-and Other-rated Scales by Primary Diagnosis

\begin{tabular}{|c|c|c|c|c|c|c|}
\hline Scale* & Ethnicity & $n$ & M & $\mathrm{SE}$ & SD & $\begin{array}{c}\text { Group } \\
\text { Differences }\end{array}$ \\
\hline S-CAARS A & No Diagnosis & 129 & $55.33^{\mathrm{a}, \mathrm{b}}$ & 1.13 & 12.80 & \\
\hline Inattention/ & ADHD-C & 365 & $69.97^{\mathrm{d}}$ & 0.56 & 10.69 & \\
\hline Memory & ADHD-I & 282 & $69.24^{\mathrm{d}}$ & 0.59 & 9.98 & \\
\hline \multirow[t]{5}{*}{ Problems } & Anxiety & 61 & $57.87^{\mathrm{a}, \mathrm{b}, \mathrm{c}}$ & 1.65 & 12.91 & \\
\hline & Depression & 40 & $62.70^{\mathrm{c}}$ & 1.71 & 10.79 & \\
\hline & NVLD & 105 & $59.95^{\mathrm{b}, \mathrm{c}}$ & 1.49 & 15.24 & \\
\hline & VLD & 145 & $53.23^{\mathrm{a}}$ & 1.03 & 12.44 & $F(6,1120)=$ \\
\hline & Total & 1127 & 64.11 & 0.40 & 13.42 & $63.25, p<.001$ \\
\hline S-CAARS B & No Diagnosis & 129 & $48.88^{\mathrm{a}}$ & 1.04 & 11.80 & \\
\hline Hyperactivity/ & ADHD-C & 365 & $64.42^{\mathrm{b}}$ & 0.51 & 9.67 & \\
\hline \multirow[t]{6}{*}{ Restlessness } & ADHD-I & 282 & $52.87^{\mathrm{a}}$ & 0.54 & 9.03 & \\
\hline & Anxiety & 61 & $49.69^{\mathrm{a}}$ & 1.55 & 12.13 & \\
\hline & Depression & 40 & $50.98^{\mathrm{a}}$ & 2.02 & 12.79 & \\
\hline & NVLD & 105 & $51.44^{\mathrm{a}}$ & 1.16 & 11.88 & \\
\hline & VLD & 145 & $49.73^{\mathrm{a}}$ & 0.86 & 10.34 & $F(6,1120)=$ \\
\hline & Total & 1127 & 55.38 & 0.36 & 12.14 & $71.62, p<.001$ \\
\hline S-CAARS C & No Diagnosis & 129 & $46.54^{\mathrm{a}}$ & 1.04 & 11.81 & \\
\hline Impulsivity/ & ADHD-C & 365 & $59.53^{\mathrm{c}}$ & 0.61 & 11.70 & \\
\hline Emotional & ADHD-I & 282 & $53.52^{\mathrm{b}}$ & 0.66 & 11.06 & \\
\hline \multirow[t]{5}{*}{ Lability } & Anxiety & 61 & $49.25^{\mathrm{a}, \mathrm{b}}$ & 1.43 & 11.19 & \\
\hline & Depression & 40 & $50.85^{\mathrm{a}, \mathrm{b}}$ & 1.92 & 12.14 & \\
\hline & NVLD & 105 & $52.95^{\mathrm{b}}$ & 1.31 & 13.38 & \\
\hline & VLD & 145 & $46.54^{\mathrm{a}}$ & 0.85 & 10.26 & $F(6,1120)=$ \\
\hline & Total & 1127 & 53.39 & 0.37 & 12.54 & $34.98, p<.001$ \\
\hline S-CAARS D & No Diagnosis & 129 & $51.19^{\mathrm{a}, \mathrm{b}}$ & 1.07 & 12.12 & \\
\hline Problems & ADHD-C & 365 & $55.92^{\mathrm{b}, \mathrm{c}}$ & 0.60 & 11.43 & \\
\hline with Self & ADHD-I & 282 & $58.06^{\mathrm{c}}$ & 0.68 & 11.34 & \\
\hline \multirow[t]{5}{*}{ Concept } & Anxiety & 61 & $55.03^{\mathrm{a}, \mathrm{b}, \mathrm{c}}$ & 1.54 & 12.05 & \\
\hline & Depression & 40 & $60.25^{\mathrm{c}}$ & 1.68 & 10.65 & \\
\hline & NVLD & 105 & $56.08^{b, c}$ & 1.28 & 13.10 & \\
\hline & VLD & 145 & $49.74^{\mathrm{a}}$ & 0.93 & 11.20 & $F(6,1120)=$ \\
\hline & Total & 1127 & 55.24 & 0.36 & 11.97 & $12.30, p<.001$ \\
\hline
\end{tabular}


Table 43

WAIS-III/IV Combined Dataset ANOVA Results for the CAARS Self-and Other-rated Scales by Primary Diagnosis, continued

\begin{tabular}{|c|c|c|c|c|c|c|}
\hline Scale* & Ethnicity & $n$ & M & $\mathrm{SE}$ & SD & $\begin{array}{c}\text { Group } \\
\text { Differences }\end{array}$ \\
\hline $\begin{array}{l}\text { S-CAARS E } \\
\text { DSM-IV } \\
\text { Inattentive } \\
\text { Symptoms }\end{array}$ & $\begin{array}{l}\text { No Diagnosis } \\
\text { ADHD-C } \\
\text { ADHD-I } \\
\text { Anxiety } \\
\text { Depression } \\
\text { NVLD } \\
\text { VLD } \\
\text { Total }\end{array}$ & $\begin{array}{r}129 \\
365 \\
282 \\
61 \\
40 \\
105 \\
145 \\
1127\end{array}$ & $\begin{array}{l}63.16^{\mathrm{a}} \\
80.40^{\mathrm{c}} \\
78.97^{\mathrm{c}} \\
64.56^{\mathrm{a}} \\
71.25^{\mathrm{b}} \\
67.31^{\mathrm{a}, \mathrm{b}} \\
61.08^{\mathrm{a}} \\
73.18\end{array}$ & $\begin{array}{l}1.43 \\
0.51 \\
0.58 \\
1.89 \\
2.13 \\
1.75 \\
1.25 \\
0.45\end{array}$ & $\begin{array}{r}16.19 \\
9.77 \\
9.74 \\
14.75 \\
13.49 \\
17.91 \\
15.05 \\
14.96\end{array}$ & $\begin{array}{c}F(6,1120)= \\
73.20, p<.001\end{array}$ \\
\hline $\begin{array}{l}\text { S-CAARS F } \\
\text { DSM-IV } \\
\text { Hyperactive/ } \\
\text { Impulsive } \\
\text { Symptoms }\end{array}$ & $\begin{array}{l}\text { No Diagnosis } \\
\text { ADHD-C } \\
\text { ADHD-I } \\
\text { Anxiety } \\
\text { Depression } \\
\text { NVLD } \\
\text { VLD } \\
\text { Total }\end{array}$ & $\begin{array}{r}129 \\
365 \\
282 \\
61 \\
40 \\
105 \\
144 \\
1126\end{array}$ & $\begin{array}{l}48.67^{\mathrm{a}} \\
69.17^{\mathrm{c}} \\
56.17^{\mathrm{b}} \\
48.93^{\mathrm{a}} \\
52.23^{\mathrm{a}, \mathrm{b}} \\
54.55^{\mathrm{a}, \mathrm{b}} \\
49.67^{\mathrm{a}, \mathrm{b}} \\
58.01\end{array}$ & $\begin{array}{l}1.22 \\
0.66 \\
0.70 \\
1.81 \\
2.12 \\
1.59 \\
1.09 \\
0.46\end{array}$ & $\begin{array}{l}13.83 \\
12.65 \\
11.70 \\
14.17 \\
13.38 \\
16.33 \\
13.10 \\
15.43\end{array}$ & $\begin{array}{c}F(6,1119)= \\
72.96, p<.001\end{array}$ \\
\hline $\begin{array}{l}\text { S-CAARS G } \\
\text { DSM-IV } \\
\text { ADHD } \\
\text { Symptoms } \\
\text { Total }\end{array}$ & $\begin{array}{l}\text { No Diagnosis } \\
\text { ADHD-C } \\
\text { ADHD-I } \\
\text { Anxiety } \\
\text { Depression } \\
\text { NVLD } \\
\text { VLD } \\
\text { Total }\end{array}$ & $\begin{array}{r}129 \\
365 \\
282 \\
61 \\
40 \\
105 \\
144 \\
1126\end{array}$ & $\begin{array}{l}57.63^{\mathrm{a}} \\
78.98^{\mathrm{d}} \\
71.84^{\mathrm{c}} \\
58.36^{\mathrm{a}} \\
65.15^{\mathrm{b}} \\
63.25^{\mathrm{a}, \mathrm{b}} \\
57.14^{\mathrm{a}} \\
68.88\end{array}$ & $\begin{array}{l}1.42 \\
0.54 \\
0.63 \\
1.83 \\
2.04 \\
1.75 \\
1.23 \\
0.46\end{array}$ & $\begin{array}{l}16.11 \\
10.27 \\
10.53 \\
14.27 \\
12.90 \\
17.94 \\
14.72 \\
15.56\end{array}$ & $\begin{array}{c}F(6,1119)= \\
86.83, p<.001\end{array}$ \\
\hline $\begin{array}{l}\text { S-CAARS H } \\
\text { ADHD } \\
\text { Index }\end{array}$ & $\begin{array}{l}\text { No Diagnosis } \\
\text { ADHD-C } \\
\text { ADHD-I } \\
\text { Anxiety } \\
\text { Depression } \\
\text { NVLD } \\
\text { VLD } \\
\text { Total }\end{array}$ & $\begin{array}{r}129 \\
365 \\
282 \\
61 \\
40 \\
105 \\
144 \\
1126\end{array}$ & $\begin{array}{l}52.17^{\mathrm{a}} \\
66.01^{\mathrm{d}} \\
61.32^{\mathrm{c}, \mathrm{d}} \\
54.79^{\mathrm{a}, \mathrm{b}} \\
58.90^{\mathrm{b}, \mathrm{c}} \\
56.30^{\mathrm{a}, \mathrm{b}, \mathrm{c}} \\
51.35^{\mathrm{a}} \\
59.61\end{array}$ & $\begin{array}{l}1.05 \\
0.45 \\
0.52 \\
1.41 \\
1.72 \\
1.28 \\
0.90 \\
0.34\end{array}$ & $\begin{array}{r}11.89 \\
8.56 \\
8.74 \\
11.03 \\
10.89 \\
13.08 \\
10.80 \\
11.47\end{array}$ & $\begin{array}{c}F(6,1119)= \\
58.47, p<.001\end{array}$ \\
\hline
\end{tabular}


Table 43

WAIS-III/IV Combined Dataset ANOVA Results for the CAARS Self-and Other-rated Scales by Primary Diagnosis, continued

\begin{tabular}{|c|c|c|c|c|c|c|}
\hline Scale* & Ethnicity & $n$ & M & $\mathrm{SE}$ & SD & $\begin{array}{c}\text { Group } \\
\text { Differences }\end{array}$ \\
\hline $\begin{array}{l}\text { O-CAARS A } \\
\text { Inattention/ } \\
\text { Memory } \\
\text { Problems }\end{array}$ & $\begin{array}{l}\text { No Diagnosis } \\
\text { ADHD-C } \\
\text { ADHD-I } \\
\text { Anxiety } \\
\text { Depression } \\
\text { NVLD } \\
\text { VLD } \\
\text { Total }\end{array}$ & $\begin{array}{r}77 \\
221 \\
215 \\
39 \\
28 \\
50 \\
62 \\
692\end{array}$ & $\begin{array}{l}52.74^{\mathrm{a}} \\
67.11^{\mathrm{c}} \\
66.27^{\mathrm{b}, \mathrm{c}} \\
54.28^{\mathrm{a}} \\
56.57^{\mathrm{a}} \\
58.54^{\mathrm{a}, \mathrm{b}} \\
51.52^{\mathrm{a}} \\
62.08\end{array}$ & $\begin{array}{l}1.44 \\
0.80 \\
0.78 \\
2.07 \\
2.75 \\
2.24 \\
1.64 \\
0.53\end{array}$ & $\begin{array}{l}12.63 \\
11.87 \\
11.41 \\
12.93 \\
14.54 \\
15.82 \\
12.89 \\
13.83\end{array}$ & $\begin{array}{c}F(6,685)= \\
29.07, p<.001\end{array}$ \\
\hline $\begin{array}{l}\text { O-CAARS B } \\
\text { Hyperactivity/ } \\
\text { Restlessness }\end{array}$ & $\begin{array}{l}\text { No Diagnosis } \\
\text { ADHD-C } \\
\text { ADHD-I } \\
\text { Anxiety } \\
\text { Depression } \\
\text { NVLD } \\
\text { VLD } \\
\text { Total }\end{array}$ & $\begin{array}{r}77 \\
221 \\
215 \\
39 \\
28 \\
50 \\
62 \\
692\end{array}$ & $\begin{array}{l}47.16^{\mathrm{a}} \\
63.58^{\mathrm{b}} \\
52.29^{\mathrm{a}} \\
51.36^{\mathrm{a}} \\
48.82^{\mathrm{a}} \\
53.60^{\mathrm{a}} \\
48.71^{\mathrm{a}} \\
54.90\end{array}$ & $\begin{array}{l}1.15 \\
0.78 \\
0.74 \\
1.94 \\
2.09 \\
2.10 \\
1.40 \\
0.49\end{array}$ & $\begin{array}{l}10.12 \\
11.57 \\
10.84 \\
12.08 \\
11.04 \\
14.82 \\
11.02 \\
12.96\end{array}$ & $\begin{array}{c}F(6,685)= \\
34.11, p<.001\end{array}$ \\
\hline $\begin{array}{l}\text { O-CAARS C } \\
\text { Impulsivity/ } \\
\text { Emotional } \\
\text { Lability }\end{array}$ & $\begin{array}{l}\text { No Diagnosis } \\
\text { ADHD-C } \\
\text { ADHD-I } \\
\text { Anxiety } \\
\text { Depression } \\
\text { NVLD } \\
\text { VLD } \\
\text { Total }\end{array}$ & $\begin{array}{r}77 \\
221 \\
215 \\
39 \\
28 \\
50 \\
62 \\
692\end{array}$ & $\begin{array}{l}46.34^{\mathrm{a}} \\
57.94^{\mathrm{c}} \\
52.00^{\mathrm{a}, \mathrm{b}, \mathrm{c}} \\
48.87^{\mathrm{a}, \mathrm{b}} \\
49.79^{\mathrm{a}, \mathrm{b}} \\
53.64^{\mathrm{b}, \mathrm{c}} \\
45.68^{\mathrm{a}} \\
52.55\end{array}$ & $\begin{array}{l}1.05 \\
0.70 \\
0.68 \\
1.70 \\
2.05 \\
1.72 \\
1.02 \\
0.42\end{array}$ & $\begin{array}{r}9.23 \\
10.37 \\
9.94 \\
10.62 \\
10.85 \\
12.13 \\
8.00 \\
10.96\end{array}$ & $\begin{array}{c}F(6,685)= \\
21.55, p<.001\end{array}$ \\
\hline $\begin{array}{l}\text { O-CAARS D } \\
\text { Problems } \\
\text { with Self } \\
\text { Concept }\end{array}$ & $\begin{array}{l}\text { No Diagnosis } \\
\text { ADHD-C } \\
\text { ADHD-I } \\
\text { Anxiety } \\
\text { Depression } \\
\text { NVLD } \\
\text { VLD } \\
\text { Total }\end{array}$ & $\begin{array}{r}77 \\
221 \\
215 \\
39 \\
28 \\
50 \\
62 \\
692\end{array}$ & $\begin{array}{l}48.51^{\mathrm{a}} \\
56.16^{\mathrm{b}} \\
55.54^{\mathrm{a}, \mathrm{b}} \\
55.08^{\mathrm{a}, \mathrm{b}} \\
58.61^{\mathrm{b}} \\
57.24^{\mathrm{b}} \\
51.85^{\mathrm{a}, \mathrm{b}} \\
54.85^{-}\end{array}$ & $\begin{array}{l}1.16 \\
0.80 \\
0.79 \\
1.91 \\
2.10 \\
1.66 \\
1.56 \\
0.45\end{array}$ & $\begin{array}{l}10.19 \\
11.84 \\
11.57 \\
11.96 \\
11.10 \\
11.71 \\
12.24 \\
11.83\end{array}$ & $\begin{array}{c}F(6,685)= \\
5.98, p<.001\end{array}$ \\
\hline
\end{tabular}


Table 43

WAIS-III/IV Combined Dataset ANOVA Results for the CAARS Self-and Other-rated Scales by Primary Diagnosis, continued

\begin{tabular}{|c|c|c|c|c|c|c|}
\hline Scale* & Ethnicity & $n$ & $\mathrm{M}$ & $\mathrm{SE}$ & SD & $\begin{array}{c}\text { Group } \\
\text { Differences }\end{array}$ \\
\hline O-CAARS E & No Diagnosis & 77 & $54.26^{\mathrm{a}}$ & 1.31 & 11.53 & \\
\hline DSM-IV & ADHD-C & 221 & $67.08^{\mathrm{b}}$ & 0.69 & 10.25 & \\
\hline Inattentive & ADHD-I & 215 & $64.95^{\mathrm{b}}$ & 0.75 & 11.03 & \\
\hline \multirow[t]{5}{*}{ Symptoms } & Anxiety & 39 & $53.03^{\mathrm{a}}$ & 1.93 & 12.06 & \\
\hline & Depression & 28 & $53.07^{\mathrm{a}}$ & 2.31 & 12.21 & \\
\hline & NVLD & 50 & $56.90^{\mathrm{a}}$ & 1.81 & 12.80 & \\
\hline & VLD & 62 & $51.73^{\mathrm{a}}$ & 1.39 & 10.91 & $F(36,685)=$ \\
\hline & Total & 692 & 61.52 & 0.48 & 12.58 & $34.28, p<.001$ \\
\hline O-CAARS F & No Diagnosis & 77 & $46.96^{\mathrm{a}}$ & 1.15 & 10.07 & \\
\hline DSM-IV & ADHD-C & 221 & $63.90^{c}$ & 0.81 & 12.06 & \\
\hline Hyperactive/ & ADHD-I & 216 & $52.76^{\mathrm{a}, \mathrm{b}}$ & 0.75 & 11.08 & \\
\hline Impulsive & Anxiety & 39 & $51.26^{\mathrm{a}, \mathrm{b}}$ & 1.99 & 12.40 & \\
\hline \multirow[t]{4}{*}{ Symptoms } & Depression & 28 & $47.61^{\mathrm{a}, \mathrm{b}}$ & 2.09 & 11.04 & \\
\hline & NVLD & 50 & $54.68^{b}$ & 2.10 & 14.87 & \\
\hline & VLD & 62 & $49.02^{\mathrm{a}, \mathrm{b}}$ & 1.21 & 9.52 & $F(6,685)=$ \\
\hline & Total & 693 & 55.18 & 0.50 & 13.14 & $34.79, p<.001$ \\
\hline O-CAARS G & No Diagnosis & 77 & $50.99^{\mathrm{a}}$ & 1.28 & 11.19 & \\
\hline DSM-IV & ADHD-C & 221 & $67.78^{\mathrm{c}}$ & 0.73 & 10.87 & \\
\hline ADHD & ADHD-I & 215 & $60.48^{b}$ & 0.71 & 10.43 & \\
\hline Symptoms & Anxiety & 39 & $53.49^{\mathrm{a}, \mathrm{b}}$ & 1.98 & 12.35 & \\
\hline \multirow[t]{4}{*}{ Total } & Depression & 28 & $50.68^{\mathrm{a}}$ & 2.12 & 11.22 & \\
\hline & NVLD & 50 & $57.00^{\mathrm{a}, \mathrm{b}}$ & 2.01 & 14.18 & \\
\hline & VLD & 62 & $50.69^{\mathrm{a}}$ & 1.27 & 10.02 & $F(6,685)=$ \\
\hline & Total & 692 & 59.84 & 0.49 & 12.82 & $40.22, p<.001$ \\
\hline O-CAARS H & No Diagnosis & 77 & $49.17^{\mathrm{a}}$ & 1.22 & 10.68 & \\
\hline ADHD & ADHD-C & 221 & $64.94^{\mathrm{d}}$ & 0.71 & 10.54 & \\
\hline \multirow[t]{6}{*}{ Index } & ADHD-I & 215 & $60.20^{\mathrm{c}, \mathrm{d}}$ & 0.63 & 9.29 & \\
\hline & Anxiety & 39 & $53.67^{\mathrm{a}, \mathrm{b}, \mathrm{c}}$ & 1.74 & 10.88 & \\
\hline & Depression & 28 & $53.04^{\mathrm{a}, \mathrm{b}}$ & 2.28 & 12.05 & \\
\hline & NVLD & 50 & $57.52^{\mathrm{b}, \mathrm{c}}$ & 1.79 & 12.66 & \\
\hline & VLD & 62 & $50.71^{\mathrm{a}, \mathrm{b}}$ & 1.31 & 10.34 & $F(6,685)=$ \\
\hline & Total & 692 & 58.78 & 0.45 & 11.81 & $33.76, p<.001$ \\
\hline
\end{tabular}


Table 43

WAIS-III/IV Combined Dataset ANOVA Results for the CAARS Self-and Other-rated Scales by Primary Diagnosis, continued

Note. WAIS-III/IV = Wechsler Adult Intelligence Scale - Third/Fourth Editions. CAARS $=$ Conners Adult ADHD Rating Scale. $\mathrm{M}=$ Mean. $\mathrm{SE}=$ Standard Error of the Mean. $\mathrm{SD}=$ Standard Deviation. $\mathrm{S}=$ Self-Rated Scale. $\mathrm{O}=$ Other-Rated Scale. ADHD-C $=$ Attention Deficit Hyperactivity Disorder - Combined Type. ADHD-I = Attention Deficit Hyperactivity Disorder, Predominantly Inattentive Type. NVLD = Nonverbal Learning Disability. VLD = Verbal Learning Disability. *CAARS Rating Scales have a $\mathrm{T}$-score $\mathrm{M}=50, \mathrm{SD}=10$. Means sharing the same superscript are not significantly different from each other (Scheffé's, $p<0.05$ ).

Table 44 illustrates the results of an examination of the BDI-II and SCL-90-R by diagnosis. As expected, the mean score of the Depression group was significantly higher than the mean scores of the other diagnoses. This finding is not surprising given that the BDI-II is used to help diagnose Depression. The SCL-90-R Global Severity Index (GSI) differentiated only the Depression group, which scored in the high group, from the No Diagnosis and VLD groups. All the other diagnoses shared significance with these two groups. This finding is also not that surprising given that the GSI is simply a measure of overall psychological distress. It makes sense that anyone diagnosed with any of these disorders would experience a certain level of distress. Perhaps the interesting finding here is that the VLD group as a whole did not experience as much distress, as expressed by the GSI, as the other diagnostic groups. Also important to remember is that, for this sample group, many of the more severely distressed individuals were not administered a BDI-II or SCL-90-R but instead were given a structured clinical interview to assess their psychological symptoms. 
Table 44

WAIS-III/IV Combined Dataset ANOVA Results for the BDI-II Total Score and SCL-90-R GSI by Primary Diagnosis

\begin{tabular}{llrrrrr}
\hline Scale* & Ethnicity & $n$ & $\mathrm{M}$ & $\mathrm{SE}$ & $\mathrm{SD}$ & $\begin{array}{c}\text { Group } \\
\text { Differences }\end{array}$ \\
\hline BDI-II & No Diagnosis & 122 & $11.10^{\mathrm{a}}$ & 0.93 & 10.22 & \\
Total Score & ADHD-C & 342 & $14.55^{\mathrm{a}}$ & 0.58 & 10.68 & \\
& ADHD-I & 248 & $14.22^{\mathrm{a}}$ & 0.61 & 9.65 & \\
& Anxiety & 49 & $11.80^{\mathrm{a}}$ & 1.39 & 9.70 & \\
& Depression & 32 & $22.03^{\mathrm{b}}$ & 1.72 & 9.73 & \\
& NVLD & 96 & $14.30^{\mathrm{a}}$ & 1.08 & 10.62 & \\
& VLD & 139 & $10.85^{\mathrm{a}}$ & 0.77 & 9.04 & $F(6,1021)=$ \\
SCL-90-R & Total & 1028 & 13.64 & 0.32 & 10.29 & $7.69, p<.001$ \\
GSI & No Diagnosis & 116 & $53.85^{\mathrm{a}}$ & 1.13 & 12.11 & \\
& ADHD-C & 319 & $60.66^{\mathrm{a}, \mathrm{b}}$ & 0.69 & 12.23 & \\
& ADHD-I & 244 & $59.50^{\mathrm{a}, \mathrm{b}}$ & 0.79 & 12.40 & \\
& Anxiety & 45 & $59.47^{\mathrm{a}, \mathrm{b}}$ & 1.72 & 11.52 & \\
& Depression & 31 & $63.74^{\mathrm{b}}$ & 2.24 & 12.49 & \\
& NVLD & 86 & $59.26^{\mathrm{a}, \mathrm{b}}$ & 1.37 & 12.67 & \\
& VLD & 122 & $54.32^{\mathrm{a}}$ & 1.13 & 12.43 & $F(6,956)=$ \\
& Total & 963 & 58.66 & 0.41 & 12.57 & $8.03, p<.001$ \\
\hline
\end{tabular}

Note. WAIS-III/IV = Wechsler Adult Intelligence Scale - Third/Fourth Editions. BDI-II $=$ Beck Depression Inventory - Second Edition. SCL-90-R GSI = Symptom Checklist90-Revised Global Severity Index. $M=$ Mean. SE = Standard Error of the Mean. SD = Standard Deviation. *Cut score guidelines for the BDI-II Total score: 0-13 is considered minimal range, 14-19 is mild, 20-28 is moderate, and 29-63 is severe for symptoms of depression; the GSI has a T-score $\mathrm{M}=50, \mathrm{SD}=10$. ADHD-C $=$ Attention Deficit Hyperactivity Disorder - Combined Type. ADHD-I = Attention Deficit Hyperactivity Disorder, Predominantly Inattentive Type. NVLD = Nonverbal Learning Disability. $\mathrm{VLD}=$ Verbal Learning Disability. Means sharing the same superscript are not significantly different from each other (Scheffé's, $p<0.05$ ).

\section{Foreign Language Learning Difficulty (FLLD)}

Analyses were conducted on the mean scores of students who were assessed based on whether they had difficulty learning a foreign language and requested a "nonprimary language substitution" (i.e., be exempted from taking a foreign language class and instead take an alternative class regarding a foreign culture). The sample size of this 
group was relatively small $(\mathrm{N}=86)$, but independent sample t-tests were performed on all cognitive and achievement subtests and rating scales to compare the FLLD group to students who came to the disabilities resource center to be tested but did not request this substitution. The FLLD group consisted of 61 men and 25 women. Caucasians represented $67.4 \%$ of the group $(N=58)$, African Americans comprised $24.4 \%(N=21)$, Asians/Indians, $4.7 \%(N=4)$, Native Americans, $2.3 \%(N=2)$, and Latinos, $1.2 \%(N=$ 1). Diagnoses included VLD (39.5\%, $N=34)$, ADHD-C (17.4\%, $N=15)$, NVLD $(16.3 \%, \mathrm{~N}=14)$, ADHD-I $(15.1 \%, N=13)$, other $(7.1 \%, N=6)$, and No Diagnosis $(4.7 \%, N=4)$.

Tables $45-49$ illustrate the results of analyses concerning students with an FLLD. The FLLD students scored significantly lower on the FSIQ and GIA than the non-FLLD students. Consistent with the findings of Robinson (2001) and Sparks and colleagues (2006), they also scored significantly lower on all comprehension-knowledge subtests except Information, where their scores trended lower $(p=.064)$, and Comprehension. Working memory was also a problem, indicated by significantly lower scores by the FLLD students on the working-memory capacity subtests Digit Span, Letter-Number Sequencing, and Numbers Reversed. The FLLD group also experienced difficulties with fluid reasoning, signified by their lower scores on the fluid reasoning subtests Matrix Reasoning, Concept Formation, and Analysis-Synthesis, relative to their peers. Other lower scores included those from the visual-spatial processing subtests Planning and Spatial Relations, the long-term storage and retrieval subtest VisualAuditory Learning, and the auditory-processing subtest Sound Blending. The lower 
cognitive scores were reflected in a universally significant reduction in all achievement scores compared to the non-FLLD group. These findings are consistent with the research described in Chapter II regarding students who struggle learning a foreign language.

Table 45

Means, Standard Errors, Standard Deviations, and t-test Results for Combined WAISIII/IV FSIQ and Subtests by Foreign Language Learning Difficulty (FLLD)

\begin{tabular}{lcrrccccc}
\hline FSIQ*/ Subtest** & FLLD & $n$ & M & SE & SD & $t$ & df & $p$ \\
\hline FSIQ & No & 1104 & 112.99 & 0.41 & 13.57 & & & \\
& Yes & 79 & 108.51 & 1.48 & 13.19 & 2.84 & 1181 & .005 \\
Vocabulary & No & 1105 & 13.01 & 0.09 & 3.09 & & & \\
& Yes & 79 & 12.09 & 0.35 & 3.14 & 2.56 & 1182 & .011 \\
Similarities & No & 1105 & 12.12 & 0.09 & 3.00 & & & \\
& Yes & 79 & 11.38 & 0.36 & 3.21 & 2.11 & 1182 & .035 \\
Arithmetic & No & 1105 & 11.97 & 0.08 & 2.71 & & & \\
& Yes & 79 & 11.53 & 0.33 & 2.89 & 1.38 & 1182 & .167 \\
Digit Span & No & 1105 & 11.02 & 0.09 & 2.95 & & & \\
& Yes & 79 & 10.24 & 0.29 & 2.56 & 2.30 & 1182 & .022 \\
Information & No & 1105 & 12.73 & 0.08 & 2.76 & & & \\
& Yes & 79 & 12.14 & 0.30 & 2.63 & 1.85 & 1182 & .064 \\
Comprehension & No & 1104 & 12.65 & 0.09 & 2.89 & & & \\
& Yes & 77 & 12.49 & 0.33 & 2.93 & 0.46 & 1179 & .644 \\
Letter-Number & No & 1105 & 11.32 & 0.10 & 3.14 & & & \\
Sequencing & Yes & 79 & 10.33 & 0.32 & 2.81 & 2.74 & 1182 & .006 \\
Picture & No & 1103 & 10.19 & 0.09 & 3.06 & & & \\
Completion & Yes & 79 & 9.76 & 0.32 & 2.87 & 1.22 & 1180 & .224 \\
Coding & No & 1105 & 10.53 & 0.09 & 2.90 & & & \\
& Yes & 79 & 9.48 & 0.29 & 2.60 & 3.12 & 1182 & .002 \\
Block Design & No & 1105 & 11.84 & 0.10 & 3.21 & & & \\
& Yes & 79 & 11.39 & 0.37 & 3.28 & 1.19 & 1182 & .234 \\
Matrix & No & 1104 & 12.88 & 0.08 & 2.61 & & & \\
Reasoning & Yes & 79 & 12.10 & 0.31 & 2.76 & 2.54 & 1181 & .011 \\
Picture & No & 873 & 11.39 & 0.10 & 2.87 & & & \\
Arrangement & Yes & 61 & 11.28 & 0.35 & 2.73 & 0.30 & 932 & .763 \\
Visual Puzzles & No & 229 & 11.04 & 0.18 & 2.77 & & & \\
& Yes & 18 & 10.78 & 0.98 & 4.17 & 0.37 & 245 & .711
\end{tabular}


Table 45

Means, Standard Errors, Standard Deviations, and t-test Results for Combined WAISIII/IV FSIQ and Subtests by Foreign Language Learning Difficulty (FLLD), continued

\begin{tabular}{lcrrrrrrr} 
FSIQ*/ Subtest** & FLLD & \multicolumn{1}{c}{$n$} & M & SE & SD & $t$ & df & $p$ \\
\hline Figure Weights & No & 228 & 12.56 & 0.19 & 2.88 & & & \\
& Yes & 18 & 11.33 & 0.79 & 3.36 & 1.72 & 244 & .087 \\
Symbol Search & No & 1104 & 10.98 & 0.09 & 3.02 & & & \\
& Yes & 79 & 10.59 & 0.31 & 2.79 & 1.10 & 1181 & .270 \\
Cancellation & No & 64 & 9.67 & 0.39 & 3.12 & & & \\
& Yes & 3 & 8.33 & 0.33 & 0.58 & 0.74 & 65 & .463 \\
\hline
\end{tabular}

Note. WAIS-III/IV = Wechsler Adult Intelligence Scale - Third/Fourth Editions.

FSIQ = Full-Scale Intelligence Quotient. SE = Standard Error of the Mean. SD = Standard Deviation. $\mathrm{df}=$ degrees of freedom. *WAIS-III/IV FSIQs have a Standard Score $\mathrm{M}=100, \mathrm{SD}=15$. **Subtests have an Index Score $\mathrm{M}=10, \mathrm{SD}=3$. Students are categorized as either requesting ("Yes") or not requesting ("No") a non-primary language substitution.

Table 46

Means, Standard Errors, Standard Deviations, and t-test Results for Combined WAISIII/IV Dataset's WJ III GIA and Subtests by Foreign Language Learning Difficulty (FLLD)

\begin{tabular}{lcrrrrrrr}
\hline GIA/ Subtest* & FLLD & $n$ & $\mathrm{M}$ & SE & SD & $t$ & df & $p$ \\
\hline GIA & No & 1084 & 103.45 & 0.37 & 12.12 & & & \\
& Yes & 80 & 96.63 & 1.31 & 11.70 & 4.87 & 1162 & $<.001$ \\
Verbal & No & 985 & 103.68 & 0.39 & 12.19 & & & \\
Comprehension & Yes & 73 & 98.74 & 1.48 & 12.64 & 3.34 & 1056 & .001 \\
Visual-Auditory & No & 1084 & 99.17 & 0.56 & 18.45 & & & \\
Learning & Yes & 80 & 92.25 & 2.08 & 18.59 & 3.24 & 1162 & .001 \\
Spatial & No & 980 & 105.37 & 0.35 & 11.08 & & & \\
Relations & Yes & 74 & 101.97 & 1.45 & 12.50 & 2.52 & 1052 & .012 \\
Sound & No & 1083 & 104.55 & 0.39 & 12.93 & & & \\
Blending & Yes & 80 & 98.53 & 1.37 & 12.24 & 4.04 & 1161 & $<.001$ \\
Concept & No & 1085 & 107.49 & 0.35 & 11.65 & & & \\
Formation & Yes & 80 & 104.20 & 1.89 & 16.94 & 2.35 & 1163 & .019 \\
Visual & No & 1087 & 101.17 & 0.48 & 15.74 & & & \\
Matching & Yes & 81 & 98.17 & 1.67 & 15.01 & 1.66 & 1166 & .097
\end{tabular}


Table 46

Means, Standard Errors, Standard Deviations, and t-test Results for Combined WAIS-III/IV Dataset's WJ III GIA and Subtests by Foreign Language Learning Difficulty (FLLD), continued

\begin{tabular}{lcrrrrrrr}
\hline GIA/ Subtest* & FLLD & $n$ & M & SE & SD & $t$ & df & $p$ \\
\hline Numbers & No & 923 & 103.93 & 0.48 & 14.58 & & & \\
Reversed & Yes & 66 & 98.11 & 2.09 & 16.94 & 2.72 & 72 & .008 \\
Incomplete & No & 1073 & 106.06 & 0.45 & 14.81 & & & \\
Words & Yes & 81 & 103.60 & 1.31 & 11.75 & 1.78 & 100 & .079 \\
Retrieval & No & 981 & 97.71 & 0.36 & 11.38 & & & \\
Fluency & Yes & 74 & 97.24 & 1.11 & 9.51 & 0.34 & 1053 & .732 \\
Picture & No & 1080 & 101.67 & 0.37 & 12.04 & & & \\
Recognition & Yes & 80 & 100.08 & 1.20 & 10.70 & 1.15 & 1158 & .250 \\
Analysis- & No & 1082 & 109.98 & 0.41 & 13.50 & & & \\
Synthesis & Yes & 81 & 101.20 & 1.32 & 11.90 & 5.69 & 1161 & $<.001$ \\
Decision & No & 982 & 101.62 & 0.52 & 16.28 & & & \\
Speed & Yes & 74 & 100.77 & 1.75 & 15.05 & 0.43 & 1054 & .665 \\
Memory for & No & 1083 & 103.33 & 0.83 & 27.43 & & & \\
Words & Yes & 79 & 97.89 & 1.39 & 12.37 & 1.75 & 1160 & .080 \\
Rapid Picture & No & 976 & 96.34 & 0.52 & 16.38 & & & \\
Naming & Yes & 75 & 95.57 & 1.99 & 17.24 & 0.39 & 1049 & .696 \\
Planning & No & 974 & 113.18 & 0.79 & 24.66 & & & \\
Pair & Yes & 73 & 106.41 & 1.80 & 15.35 & 3.45 & 102 & .001 \\
Cancellation & No & 982 & 99.51 & 0.47 & 14.82 & & & \\
\hline Yes & 74 & 98.08 & 1.68 & 14.47 & 0.80 & 1054 & .425 \\
\hline
\end{tabular}

Note. WAIS-III/IV $=$ Wechsler Adult Intelligence Scale - Third/Fourth Editions. $\mathrm{SE}=$ Standard Error of the Mean. $\mathrm{SD}=$ Standard Deviation. $\mathrm{df}=$ degrees of freedom. GIA $=$ General Intellectual Ability. $*$ WJ III has a Standard Score M= $100, \mathrm{SD}=15$. Students are categorized as either requesting ("Yes") or not requesting ("No") a non-primary language substitution. 
Table 47

Means, Standard Errors, Standard Deviations, and t-test Results for Combined WAIS-III/IV Dataset's WJ III Achievement Subtests by Foreign Language Learning Difficulty (FLLD)

\begin{tabular}{|c|c|c|c|c|c|c|c|c|}
\hline Subtest* & FLLD & $n$ & M & $\mathrm{SE}$ & $\mathrm{SD}$ & $t$ & df & $p$ \\
\hline \multirow{2}{*}{$\begin{array}{l}\text { Letter-Word } \\
\text { Identification }\end{array}$} & No & 1093 & 104.37 & 0.33 & 10.75 & & & \\
\hline & Yes & 80 & 98.29 & 1.00 & 8.97 & 4.94 & 1171 & $<.001$ \\
\hline \multirow{2}{*}{$\begin{array}{l}\text { Reading } \\
\text { Fluency }\end{array}$} & No & 986 & 101.70 & 0.47 & 14.64 & & & \\
\hline & Yes & 73 & 95.74 & 1.49 & 12.73 & 3.39 & 1057 & .001 \\
\hline \multirow[t]{2}{*}{ Calculation } & No & 1085 & 111.59 & 0.47 & 15.37 & & & \\
\hline & Yes & 79 & 102.19 & 1.55 & 13.75 & 5.29 & 1162 & $<.001$ \\
\hline \multirow[t]{2}{*}{ Math Fluency } & No & 985 & 100.76 & 0.45 & 14.02 & & & \\
\hline & Yes & 73 & 95.26 & 1.56 & 13.33 & 3.24 & 1056 & .001 \\
\hline \multirow[t]{2}{*}{ Spelling } & No & 987 & 107.10 & 0.32 & 10.17 & & & \\
\hline & Yes & 73 & 99.96 & 1.21 & 10.29 & 5.78 & 1058 & $<.001$ \\
\hline \multirow{2}{*}{$\begin{array}{l}\text { Writing } \\
\text { Fluency }\end{array}$} & No & 1078 & & & & & & \\
\hline & Yes & 79 & 100.96 & 1.50 & 13.32 & 4.14 & 1155 & $<.001$ \\
\hline \multirow{2}{*}{$\begin{array}{l}\text { Passage } \\
\text { Comprehension }\end{array}$} & No & 1091 & 105.05 & 0.33 & 10.81 & & & \\
\hline & Yes & 80 & 101.09 & 1.12 & 10.01 & 3.18 & 1169 & .001 \\
\hline \multirow{2}{*}{$\begin{array}{l}\text { Applied } \\
\text { Problems }\end{array}$} & No & 1089 & 107.48 & 0.40 & 13.23 & & & \\
\hline & Yes & 79 & 100.84 & 1.21 & 10.71 & 4.36 & 1166 & $<.001$ \\
\hline \multirow{2}{*}{$\begin{array}{l}\text { Writing } \\
\text { Samples }\end{array}$} & No & 1092 & 109.05 & 0.49 & 16.07 & & & \\
\hline & Yes & 80 & 102.66 & 1.73 & 15.43 & 3.44 & 1170 & .001 \\
\hline \multirow[t]{2}{*}{ Word Attack } & No & 1066 & 102.44 & 0.34 & 11.14 & & & \\
\hline & Yes & 79 & 95.61 & 1.19 & 10.54 & 5.28 & 1143 & $<.001$ \\
\hline
\end{tabular}

Note. WAIS-III/IV = Wechsler Adult Intelligence Scale - Third/Fourth Editions. $\mathrm{M}$ $=$ Mean. $\mathrm{SE}=$ Standard Error of the Mean. $\mathrm{SD}=$ Standard Deviation. $\mathrm{df}=$ degrees of freedom. *WJ III has a Standard Score $\mathrm{M}=100, \mathrm{SD}=15$. Students are categorized as either requesting ("Yes") or not requesting ("No") a non-primary language substitution.

Tables 48 and 49 exhibit the results regarding the social-emotional functioning of students with FLLD. The FLLD students scored significantly lower on all CAARS subscales than their non-FLLD counterparts, indicating that, as a group, they typically do not experience as much ADHD-type difficulty as the non-FLLD group does. This finding is interesting considering that approximately one third of the FLLD group was 
diagnosed with ADHD. Only the mean subscale scores from the DSM-IV Inattentive

Symptoms and DSM-IV ADHD symptoms reached "At Risk" status with mean scores of $65.00(S D=16.97)$ and $61.94(S D=17.42)$, respectively.

Table 48

Means, Standard Errors, Standard Deviations, and t-test Results of the CAARS Selfand Other-rated Scales of the Combined WAIS-III/IV Dataset by Foreign Language Learning Difficulty (FLLD)

\begin{tabular}{lcrcccccc}
\hline Scale* & FLLD & \multicolumn{1}{c}{$n$} & M & SE & SD & $t$ & df & $p$ \\
\hline S-A - Inattention/ & No & 1105 & 64.36 & 0.40 & 13.38 & & & \\
Memory Problems & Yes & 78 & 56.36 & 1.51 & 13.36 & 5.10 & 1181 & $<.001$ \\
S-B - Hyperactivity/ & No & 1105 & 55.54 & 0.37 & 12.14 & & & \\
Restlessness & Yes & 78 & 50.10 & 1.32 & 11.68 & 3.83 & 1181 & $<.001$ \\
S-C - Impulsivity/ & No & 1105 & 53.61 & 0.38 & 12.67 & & & \\
Emotional Lability & Yes & 78 & 47.90 & 1.21 & 10.71 & 3.88 & 1181 & $<.001$ \\
S-D - Problems with & No & 1105 & 55.54 & 0.36 & 11.81 & & & \\
Self Concept & Yes & 78 & 50.99 & 1.51 & 13.36 & 3.26 & 1181 & .001 \\
S-E - DSM-IV & No & 1105 & 73.38 & 0.45 & 14.91 & & & \\
Inattentive Symptoms & Yes & 78 & 65.00 & 1.92 & 16.97 & 4.75 & 1181 & $<.001$ \\
S-F - DSM-IV Hyperactive & No & 1105 & 58.05 & 0.46 & 15.35 & & & \\
Impulsive Symptoms & Yes & 77 & 53.58 & 1.87 & 16.39 & 2.46 & 1180 & .014 \\
S-G - DSM-IV & No & 1105 & 69.02 & 0.47 & 15.47 & & & \\
ADHD Symptoms & Yes & 77 & 61.94 & 1.99 & 17.42 & 3.48 & 85 & .001 \\
S-H - ADHD & No & 1105 & 59.83 & 0.34 & 11.40 & & & \\
Index & Yes & 77 & 53.26 & 1.37 & 11.98 & 4.87 & 1180 & $<.001$ \\
O-A - Inattention/ & No & 690 & 54.88 & 0.52 & 13.76 & & & \\
Memory Problems & Yes & 39 & 50.08 & 2.39 & 14.95 & 3.98 & 727 & $<.001$ \\
O-B - Hyperactivity/ & No & 690 & 52.53 & 0.49 & 12.94 & & & \\
Restlessness & Yes & 39 & 48.49 & 2.20 & 13.71 & 2.25 & 727 & .025 \\
O-C - Impulsivity/ & No & 690 & 55.05 & 0.42 & 11.15 & & & \\
Emotional Lability & Yes & 39 & 50.03 & 1.37 & 8.53 & 2.23 & 727 & .026 \\
O-D - Problems with & No & 690 & 61.59 & 0.45 & 11.76 & & & \\
Self Concept & Yes & 39 & 54.00 & 2.07 & 12.90 & 2.58 & 727 & .010 \\
O- E - DSM-IV & No & 690 & 55.08 & 0.48 & 12.58 & & & \\
Inattentive Symptoms & Yes & 39 & 51.28 & 1.89 & 11.82 & 3.67 & 727 & $<.001$ \\
& & & & & & & &
\end{tabular}


Table 48

Means, Standard Errors, Standard Deviations, and t-test Results of the CAARS Selfand Other-rated Scales of the Combined WAIS-III/IV Dataset by Foreign Language Learning Difficulty (FLLD), continued

\begin{tabular}{lcrcccccc}
\hline Scale* & FLLD & \multicolumn{1}{c}{$n$} & M & SE & SD & $t$ & df & $p$ \\
\hline O-F - DSM-IV Hyperactive & No & 691 & 59.82 & 0.50 & 13.20 & & & \\
Impulsive Symptoms & Yes & 39 & 53.36 & 1.73 & 10.81 & 2.11 & 45 & .041 \\
O-G - DSM-IV & No & 690 & 58.68 & 0.49 & 12.88 & & & \\
ADHD Symptoms & Yes & 39 & 54.88 & 1.71 & 10.67 & 3.07 & 727 & .002 \\
O-H ADHD & No & 690 & 50.08 & 0.45 & 11.79 & & & \\
Index & Yes & 39 & 52.53 & 2.07 & 12.90 & 2.18 & 727 & .030 \\
\hline
\end{tabular}

Note. $\mathrm{SE}=$ Standard Error of the Mean. $\mathrm{S}=$ Self-rated scale. $\mathrm{O}=$ Other-rated scale. *CAARS Rating Scales have a T-score $\mathrm{M}=50, \mathrm{SD}=10$. Students are categorized as either requesting ("Yes") or not requesting ("No") a non-primary language substitution.

As can be seen in Table 49, The FLLD group trended lower with respect to their mean score on the BDI-II $(M=11.30, S D=8.71)$, relative to their peers $(M=13.81, S D$ $=10.41), t(1076)=1.95, p=.051$. The SCL-90-R GSI mean score did not significantly differ from the mean score of the non-FLLD group. In sum, although the FLLD group faces obvious difficulties with respect to their cognitive challenges and lowered achievement compared to the non-FLLD group, they appear to face fewer socialemotional and behavioral challenges.

Table 49

Means, Standard Errors, Standard Deviations, and t-test Results of the Combined WAIS-III/IV Dataset's BDI-II Total Score and SCL-90-R GSI by Foreign Language Learning Difficulty (FLLD)

\begin{tabular}{lcrccrccc}
\hline Scale* & FLLD & \multicolumn{1}{c}{$n$} & M & SE & \multicolumn{1}{c}{ SD } & $t$ & df & $p$ \\
\hline BDI-II & No & 1009 & 13.81 & 0.33 & 10.41 & & & \\
$\quad$ Total Score & Yes & 69 & 11.30 & 1.05 & 8.71 & 1.95 & 1076 & .051 \\
SCL-90-R & No & 944 & 58.61 & 0.41 & 12.56 & & & \\
$\quad$ GSI & Yes & 67 & 58.93 & 1.64 & 13.40 & -0.20 & 1009 & .843 \\
\hline
\end{tabular}


Table 49

Means, Standard Errors, Standard Deviations, and t-test Results of the Combined WAIS-III/IV Dataset's BDI-II Total Score and SCL-90-R GSI by Foreign Language Learning Difficulty (FLLD), continued

Note. WAIS-III/IV = Wechsler Adult Intelligence Scale - Third/Fourth Editions. BDI$\mathrm{II}=$ Beck Depression Inventory - Second Edition. SCL-90-R GSI = Symptom Checklist-90-Revised Global Severity Index. $\mathrm{M}=$ Mean. $\mathrm{SE}=$ Standard Error of the Mean. SD $=$ Standard Deviation. $\mathrm{df}=$ degrees of freedom. ${ }^{*}$ Cut score guidelines for the BDI-II Total Score: 0-13 is considered minimal range, 14-19 is mild, 20-28 is moderate, and 29-63 is severe for symptoms of depression; the GSI has a T-score $\mathrm{M}=50, \mathrm{SD}=10$.

\section{Hypothesis 1 - Factor Analyses of the WAIS-III, WAIS-IV, and WJ III}

The nature of the dataset required several decisions regarding how best to analyze the data with integrity while optimizing model fit. First, it was determined that combining the WAIS-III and WAIS-IV datasets would provide the largest possible sample with which to explore the data. The WAIS-IV dataset, with 253 participants, was deemed too small to perform adequate exploratory or confirmatory factor analyses with the large number of model parameters being explored. It was also decided that, because the combined data set would be large enough to allow adequate exploration of model parameters, only complete observations would be used to avoid potential difficulties with missing data. Data appeared to be missing completely at random: examiner error in failing to administer subtests, subtest scores not entered into the database because of administration or scoring error, or subtests missing because a student did not completely finish the evaluation. From an initial 1,292 sets of test scores, 889 complete observations $(68.8 \%)$ were available for the examination of the $g$ models without the achievement variables. For the models that included the achievement variables $\left(g_{f} g_{c}\right.$ and achievement predictors), 865 complete observations were used (67.0\%). 
Combining the datasets precluded using subtests not in common to both datasets; specifically, the WAIS-III subtest Picture Arrangement and the WAIS-IV subtests Figure Weights, Visual Puzzles, and Cancellation were excluded from analyses. Further, as noted in the initially hypothesized models, the WAIS cognitive subtest Arithmetic was omitted from the $g$ model but included in the $g_{f} g_{c}$ model because of its theoretically strongest loading on that model's quantitative knowledge $(\mathrm{G} q)$ factor. As a mixed and somewhat weak indicator of fluid reasoning and working memory (Weiss, Keith, Zhu, \& Chen, 2013), Arithmetic was hypothesized and primarily used as an achievement subtest loading on the quantitative knowledge $(\mathrm{G} q)$ factor.

Finally, an a priori decision was made to split the combined dataset randomly into two roughly equal halves. This split allowed a calibration-validation approach to provide the most valid opportunity for optimal model fit. The first half, labeled the calibration dataset, provided 431 complete observations (68.2\% of 632) for the $g$ models and 421 complete observations (63.8\% of 632) for the $g_{f} g_{c}$ and achievement predictor models. The calibration dataset was used to explore the data through exploratory and confirmatory factor analyses should the initial fit to the models proposed in Chapter One be less than satisfactory. The second half, labeled the validation dataset, provided 458 complete observations of 660 participants (69.4\%) for the $g$ models and 444 complete observations of 660 participants for the $g_{f} g_{c}$ and achievement predictor models. The validation dataset was used to confirm the most appropriate models suggested by the calibration dataset. Finally, both datasets were brought together to validate the final models suggested by both datasets. Cognitive and achievement subtests used in all 
analyses are listed in Table 50. All exploratory, confirmatory, and structural equation analyses were conducted with the open-source statistical software "R" (R Development Core Team, 2008), particularly with the psych (Revelle, 2013) and lavaan (Rossell, 2012) packages. Correlation matrices for cognitive and achievement variables for all three datasets are provided in Tables $14-16$. The full combined correlation matrix also includes the mental health variables used in the study. 
Table 50

Names and Abbreviations of Analyzed Cognitive and Achievement Subtests

\begin{tabular}{|c|c|c|c|}
\hline Subtest Name & $\begin{array}{c}\text { Subtest } \\
\text { Abbreviation }\end{array}$ & Subtest Name & $\begin{array}{c}\text { Subtest } \\
\text { Abbreviation }\end{array}$ \\
\hline WAIS-III/IV Subtest Name & & WJ III Subtest Name & \\
\hline Arithmetic & $\mathrm{AR}$ & Analysis-Synthesis & AS \\
\hline Block Design & $\mathrm{BD}$ & Concept Formation & $\mathrm{CF}$ \\
\hline Coding & $\mathrm{CD}$ & Decision Speed & DES \\
\hline Comprehension & $\mathrm{CO}$ & Incomplete Words & IW \\
\hline Digit Span - Backward & DSB & Memory for Words & MfW \\
\hline Digit Span - Forward & DSF & Numbers Reversed & NR \\
\hline Information & IN & Pair Cancellation & $\operatorname{PrC}$ \\
\hline Letter-Number Sequencing & LN & Picture Recognition & PR \\
\hline Matrix Reasoning & MR & Planning & PLN \\
\hline Picture Completion & $\mathrm{PC}$ & Rapid Picture Naming & RPN \\
\hline Similarities & SI & Retrieval Fluency & $\mathrm{RF}$ \\
\hline Symbol Search & SS & Sound Blending & SB \\
\hline Vocabulary & $\mathrm{VO}$ & Spatial Relations & SPR \\
\hline WJ III Achievement Subtest & & WJ III Subtest Name & \\
\hline Applied Problems & AP & Verbal Comprehension & $\mathrm{VC}$ \\
\hline Calculation & CAL & Visual-Auditory Learning & VAL \\
\hline Letter-Word Identification & LWI & Visual Matching & VM \\
\hline Math Fluency & MFL & & \\
\hline Passage Comprehension & $\mathrm{PSC}$ & & \\
\hline Reading Fluency & RFL & & \\
\hline Spelling & SP & & \\
\hline Word Attack & WA & & \\
\hline Writing Fluency & WFL & & \\
\hline Writing Samples & WS & & \\
\hline
\end{tabular}


Table 51

Calibration Dataset Correlation Matrix for Cognitive and Achievement Variables

\begin{tabular}{|c|c|c|c|c|c|c|c|c|c|c|c|c|c|}
\hline & VO & SI & IN & $\mathrm{CO}$ & $\mathrm{VC}$ & DSF & $\mathrm{DSB}$ & AR & BD & MR & VAL & SPR & PC \\
\hline VO & 1.00 & 0.60 & 0.65 & 0.61 & 0.66 & 0.20 & 0.09 & 0.38 & 0.43 & 0.36 & 0.29 & 0.37 & 0.33 \\
\hline SI & & 1.00 & 0.50 & 0.48 & 0.50 & 0.20 & 0.12 & 0.36 & 0.37 & 0.38 & 0.32 & 0.34 & 0.34 \\
\hline IN & & & 1.00 & 0.48 & 0.60 & 0.17 & 0.11 & 0.47 & 0.42 & 0.31 & 0.33 & 0.34 & 0.21 \\
\hline $\mathrm{CO}$ & & & & 1.00 & 0.48 & 0.14 & 0.12 & 0.33 & 0.31 & 0.32 & 0.25 & 0.31 & 0.34 \\
\hline $\mathrm{VC}$ & & & & & 1.00 & 0.20 & 0.18 & 0.44 & 0.49 & 0.38 & 0.41 & 0.45 & 0.35 \\
\hline DSF & & & & & & 1.00 & 0.48 & 0.23 & 0.23 & 0.27 & 0.19 & 0.16 & 0.13 \\
\hline DSB & & & & & & & 1.00 & 0.28 & 0.19 & 0.29 & 0.26 & 0.20 & 0.11 \\
\hline $\mathrm{AR}$ & & & & & & & & 1.00 & 0.51 & 0.38 & 0.29 & 0.34 & 0.21 \\
\hline $\mathrm{BD}$ & & & & & & & & & 1.00 & 0.47 & 0.38 & 0.55 & 0.39 \\
\hline MR & & & & & & & & & & 1.00 & 0.38 & 0.50 & 0.38 \\
\hline VAL & & & & & & & & & & & 1.00 & 0.42 & 0.34 \\
\hline SPR & & & & & & & & & & & & 1.00 & 0.37 \\
\hline $\mathrm{PC}$ & & & & & & & & & & & & & 1.00 \\
\hline
\end{tabular}


Table 51

Calibration Dataset Correlation Matrix for Cognitive and Achievement Variables, continued

\begin{tabular}{|c|c|c|c|c|c|c|c|c|c|c|c|c|c|}
\hline & AS & tPLN & $\mathrm{CF}$ & SB & IW & LN & NR & MfW & $\mathrm{CD}$ & SS & VM & $\mathrm{RF}$ & DES \\
\hline VO & 0.31 & 0.29 & 0.35 & 0.43 & 0.31 & 0.24 & 0.15 & 0.23 & 0.10 & 0.21 & 0.10 & 0.27 & 0.06 \\
\hline SI & 0.27 & 0.26 & 0.34 & 0.42 & 0.31 & 0.24 & 0.12 & 0.20 & 0.11 & 0.20 & 0.11 & 0.25 & 0.10 \\
\hline $\mathrm{IN}$ & 0.37 & 0.30 & 0.32 & 0.32 & 0.17 & 0.28 & 0.20 & 0.22 & 0.06 & 0.18 & 0.12 & 0.15 & 0.06 \\
\hline $\mathrm{CO}$ & 0.31 & 0.22 & 0.29 & 0.35 & 0.26 & 0.20 & 0.12 & 0.17 & 0.08 & 0.17 & 0.12 & 0.28 & 0.12 \\
\hline VC & 0.44 & 0.30 & 0.47 & 0.51 & 0.33 & 0.35 & 0.25 & 0.27 & 0.12 & 0.23 & 0.19 & 0.25 & 0.17 \\
\hline DSF & 0.10 & 0.11 & 0.13 & 0.29 & 0.18 & 0.53 & 0.48 & 0.52 & 0.07 & 0.16 & 0.14 & 0.08 & -0.03 \\
\hline DSB & 0.24 & 0.20 & 0.23 & 0.23 & 0.09 & 0.50 & 0.59 & 0.40 & 0.20 & 0.13 & 0.26 & 0.04 & 0.08 \\
\hline $\mathrm{AR}$ & 0.38 & 0.29 & 0.41 & 0.28 & 0.16 & 0.32 & 0.30 & 0.24 & 0.10 & 0.17 & 0.18 & 0.12 & 0.04 \\
\hline $\mathrm{BD}$ & 0.41 & 0.41 & 0.43 & 0.35 & 0.26 & 0.37 & 0.28 & 0.25 & 0.19 & 0.34 & 0.33 & 0.18 & 0.22 \\
\hline MR & 0.38 & 0.38 & 0.45 & 0.33 & 0.25 & 0.35 & 0.31 & 0.25 & 0.10 & 0.22 & 0.21 & 0.18 & 0.14 \\
\hline VAL & 0.37 & 0.38 & 0.40 & 0.30 & 0.26 & 0.31 & 0.30 & 0.18 & 0.15 & 0.20 & 0.22 & 0.20 & 0.16 \\
\hline SPR & 0.37 & 0.42 & 0.46 & 0.36 & 0.30 & 0.27 & 0.29 & 0.25 & 0.09 & 0.21 & 0.21 & 0.14 & 0.16 \\
\hline $\mathrm{PC}$ & 0.23 & 0.30 & 0.32 & 0.29 & 0.22 & 0.26 & 0.09 & 0.16 & 0.22 & 0.24 & 0.20 & 0.21 & 0.27 \\
\hline \multicolumn{14}{|c|}{$\begin{array}{l}\text { Note. } \text { VO = Vocabulary; SI = Similarities; IN = Information; CO = Comprehension; VC = Verbal Comprehension; DSF } \\
=\text { Digit Span - Forward; DSB = Digit Span }- \text { Backward; AR = Arithmetic; BD = Block Design; MR = Matrix } \\
\text { Reasoning; VAL = Visual-Auditory Learning; SPR = Spatial Relations; PC = Picture Completion; AS = Analysis- } \\
\text { Synthesis; tPLN = Planning with } 4^{\text {th }} \text {-Root Transformation; CF = Concept Formation; SB = Sound Blending; IW = } \\
\text { Incomplete Words; LNS = Letter-Number Sequencing; MfW = Memory for Words; CD = Coding; SS = Symbol Search; } \\
\text { VM = Visual Matching; RF = Retrieval Fluency; DES = Decision Speed. }\end{array}$} \\
\hline
\end{tabular}


Table 51

Calibration Dataset Correlation Matrix for Cognitive and Achievement Variables, continued

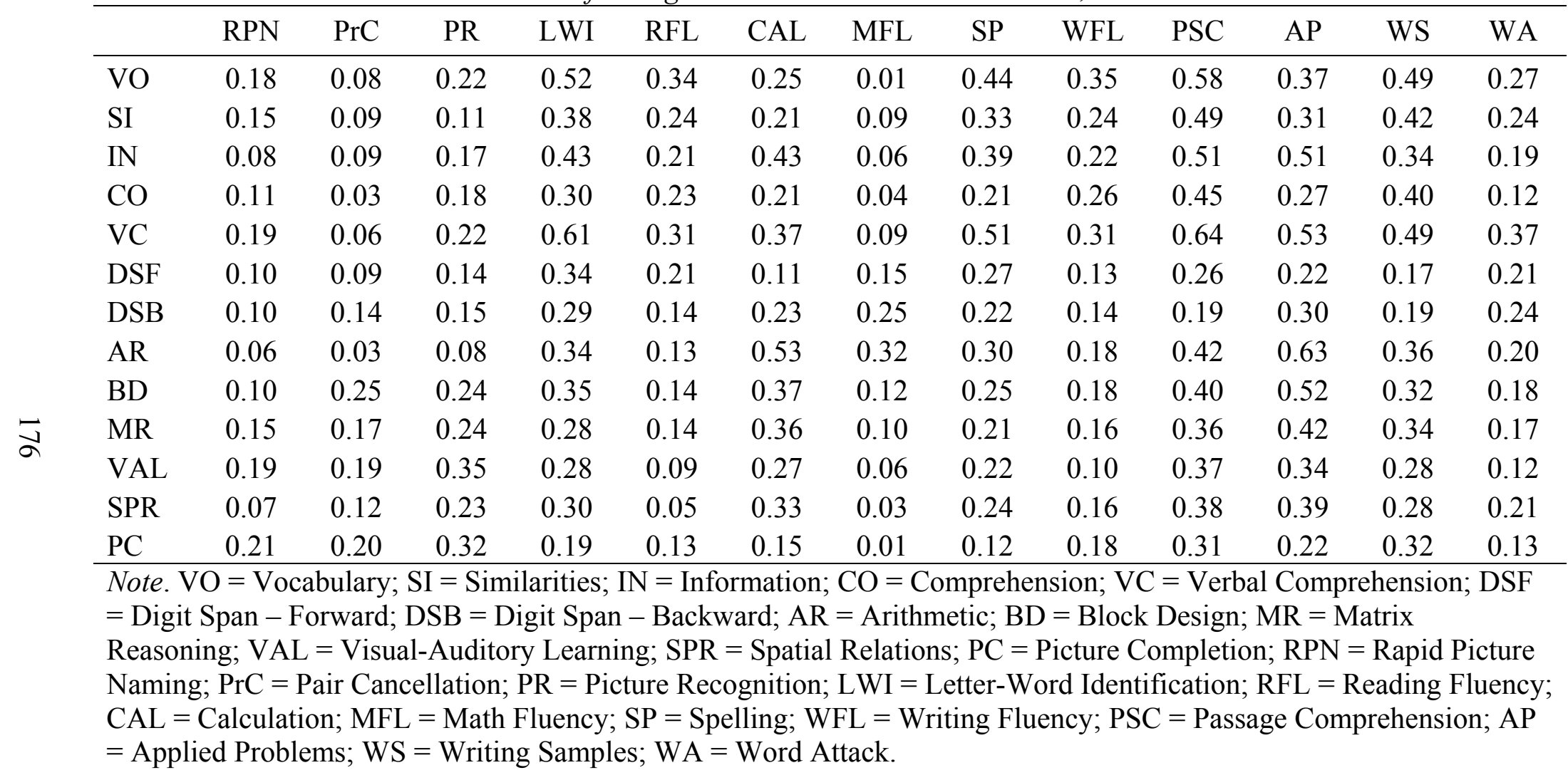


Table 51

Calibration Dataset Correlation Matrix for Cognitive and Achievement Variables, continued

\begin{tabular}{|c|c|c|c|c|c|c|c|c|c|c|c|c|c|}
\hline & AS & tPLN & $\mathrm{CF}$ & SB & IW & LN & NR & MfW & $\mathrm{CD}$ & $\mathrm{SS}$ & VM & $\mathrm{RF}$ & DES \\
\hline AS & 1.00 & 0.39 & 0.45 & 0.25 & 0.15 & 0.25 & 0.30 & 0.18 & 0.20 & 0.14 & 0.27 & 0.14 & 0.13 \\
\hline tPLN & & 1.00 & 0.39 & 0.23 & 0.18 & 0.21 & 0.26 & 0.20 & 0.12 & 0.12 & 0.22 & 0.08 & 0.10 \\
\hline $\mathrm{CF}$ & & & 1.00 & 0.32 & 0.21 & 0.32 & 0.25 & 0.22 & 0.17 & 0.21 & 0.27 & 0.14 & 0.21 \\
\hline SB & & & & 1.00 & 0.47 & 0.31 & 0.23 & 0.33 & 0.11 & 0.25 & 0.13 & 0.27 & 0.18 \\
\hline IW & & & & & 1.00 & 0.20 & 0.17 & 0.22 & 0.13 & 0.20 & 0.24 & 0.29 & 0.18 \\
\hline LN & & & & & & 1.00 & 0.51 & 0.51 & 0.20 & 0.25 & 0.27 & 0.11 & 0.12 \\
\hline NR & & & & & & & 1.00 & 0.50 & 0.16 & 0.17 & 0.26 & 0.05 & 0.06 \\
\hline MfW & & & & & & & & 1.00 & 0.11 & 0.20 & 0.20 & 0.14 & 0.08 \\
\hline $\mathrm{CD}$ & & & & & & & & & 1.00 & 0.46 & 0.51 & 0.31 & 0.45 \\
\hline SS & & & & & & & & & & 1.00 & 0.47 & 0.30 & 0.45 \\
\hline VM & & & & & & & & & & & 1.00 & 0.32 & 0.54 \\
\hline $\mathrm{RF}$ & & & & & & & & & & & & 1.00 & 0.35 \\
\hline DES & & & & & & & & & & & & & 1.00 \\
\hline
\end{tabular}


Table 51

Calibration Dataset Correlation Matrix for Cognitive and Achievement Variables, continued

\begin{tabular}{|c|c|c|c|c|c|c|c|c|c|c|c|c|c|}
\hline & RPN & $\operatorname{PrC}$ & PR & LWI & RFL & CAL & MFL & SP & WFL & PSC & $\mathrm{AP}$ & WS & WA \\
\hline $\mathrm{AS}$ & 0.09 & 0.09 & 0.15 & 0.30 & 0.15 & 0.52 & 0.21 & 0.28 & 0.19 & 0.45 & 0.50 & 0.35 & 0.17 \\
\hline tPLN & 0.13 & 0.08 & 0.16 & 0.17 & 0.12 & 0.31 & 0.05 & 0.17 & 0.08 & 0.33 & 0.37 & 0.34 & 0.12 \\
\hline $\mathrm{CF}$ & 0.08 & 0.10 & 0.26 & 0.34 & 0.16 & 0.35 & 0.09 & 0.28 & 0.24 & 0.42 & 0.45 & 0.35 & 0.23 \\
\hline SB & 0.23 & 0.09 & 0.28 & 0.45 & 0.22 & 0.18 & 0.02 & 0.32 & 0.29 & 0.44 & 0.22 & 0.39 & 0.27 \\
\hline IW & 0.25 & 0.15 & 0.25 & 0.22 & 0.22 & 0.03 & 0.04 & 0.20 & 0.18 & 0.29 & 0.11 & 0.22 & 0.18 \\
\hline $\mathrm{LN}$ & 0.15 & 0.17 & 0.21 & 0.36 & 0.26 & 0.28 & 0.21 & 0.29 & 0.21 & 0.35 & 0.33 & 0.20 & 0.19 \\
\hline NR & 0.08 & 0.13 & 0.25 & 0.34 & 0.25 & 0.25 & 0.27 & 0.30 & 0.18 & 0.26 & 0.38 & 0.24 & 0.26 \\
\hline MfW & 0.11 & 0.14 & 0.11 & 0.37 & 0.24 & 0.14 & 0.16 & 0.32 & 0.28 & 0.28 & 0.23 & 0.22 & 0.27 \\
\hline $\mathrm{CD}$ & 0.39 & 0.42 & 0.17 & 0.14 & 0.39 & 0.13 & 0.39 & 0.16 & 0.36 & 0.15 & 0.13 & 0.07 & 0.07 \\
\hline SS & 0.31 & 0.49 & 0.26 & 0.20 & 0.46 & 0.10 & 0.30 & 0.16 & 0.32 & 0.28 & 0.16 & 0.14 & 0.12 \\
\hline VM & 0.34 & 0.51 & 0.21 & 0.19 & 0.43 & 0.19 & 0.48 & 0.24 & 0.28 & 0.21 & 0.23 & 0.10 & 0.16 \\
\hline $\mathrm{RF}$ & 0.47 & 0.23 & 0.22 & 0.15 & 0.31 & 0.02 & 0.23 & 0.09 & 0.34 & 0.18 & 0.07 & 0.19 & -0.02 \\
\hline DES & 0.39 & 0.45 & 0.30 & 0.09 & 0.31 & 0.01 & 0.24 & 0.05 & 0.26 & 0.10 & 0.03 & 0.07 & 0.03 \\
\hline \multicolumn{14}{|c|}{$\begin{array}{l}\text { Note. AS = Analysis-Synthesis; tPLN = Planning with } 4^{\text {th }} \text {-Root Transformation; CF = Concept Formation; SB = Sound } \\
\text { Blending; IW = Incomplete Words; LNS = Letter-Number Sequencing; MfW = Memory for Words; CD = Coding; SS = } \\
\text { Symbol Search; VM = Visual Matching; RF = Retrieval Fluency; DES = Decision Speed; RPN = Rapid Picture Naming; } \\
\text { PrC = Pair Cancellation; PR = Picture Recognition; LWI = Letter-Word Identification; RFL = Reading Fluency; CAL = } \\
\text { Calculation; MFL = Math Fluency; SP = Spelling; WFL = Writing Fluency; PSC = Passage Comprehension; AP = } \\
\text { Applied Problems; WS = Writing Samples; WA = Word Attack. }\end{array}$} \\
\hline
\end{tabular}


Table 51

Calibration Dataset Correlation Matrix for Cognitive and Achievement Variables, continued

\begin{tabular}{|c|c|c|c|c|c|c|c|c|c|c|c|c|c|}
\hline & RPN & $\mathrm{PrC}$ & PR & LWI & RFL & CAL & MFL & SP & WFL & PSC & AP & WS & WA \\
\hline RPN & 1.00 & 0.30 & 0.22 & 0.14 & 0.44 & -0.03 & 0.24 & 0.09 & 0.31 & 0.22 & 0.01 & 0.11 & 0.01 \\
\hline $\operatorname{PrC}$ & & 1.00 & 0.16 & 0.08 & 0.28 & 0.00 & 0.31 & 0.07 & 0.24 & 0.13 & 0.03 & -0.01 & 0.06 \\
\hline PR & & & 1.00 & 0.14 & 0.13 & 0.08 & 0.04 & 0.07 & 0.14 & 0.21 & 0.09 & 0.18 & 0.05 \\
\hline LWI & & & & 1.00 & 0.34 & 0.26 & 0.17 & 0.67 & 0.39 & 0.52 & 0.42 & 0.44 & 0.58 \\
\hline RFL & & & & & 1.00 & 0.03 & 0.35 & 0.41 & 0.51 & 0.41 & 0.15 & 0.22 & 0.25 \\
\hline CAL & & & & & & 1.00 & 0.38 & 0.29 & 0.08 & 0.35 & 0.72 & 0.28 & 0.14 \\
\hline MFL & & & & & & & 1.00 & 0.27 & 0.30 & 0.18 & 0.39 & 0.08 & 0.17 \\
\hline SP & & & & & & & & 1.00 & 0.41 & 0.48 & 0.36 & 0.42 & 0.55 \\
\hline WFL & & & & & & & & & 1.00 & 0.30 & 0.15 & 0.24 & 0.27 \\
\hline PSC & & & & & & & & & & 1.00 & 0.46 & 0.44 & 0.40 \\
\hline AP & & & & & & & & & & & 1.00 & 0.37 & 0.25 \\
\hline WS & & & & & & & & & & & & 1.00 & 0.30 \\
\hline WA & & & & & & & & & & & & & 1.00 \\
\hline
\end{tabular}

Note. $\mathrm{RPN}=$ Rapid Picture Naming; PrC = Pair Cancellation; PR = Picture Recognition; LWI = Letter-Word Identification; RFL = Reading Fluency; CAL = Calculation; $\mathrm{MFL}=$ Math Fluency; $\mathrm{SP}=$ Spelling; WFL = Writing Fluency; PSC = Passage Comprehension; AP = Applied Problems; WS = Writing Samples; WA = Word Attack 
Table 52

Validation Dataset Correlation Matrix for Cognitive and Achievement Variables

\begin{tabular}{|c|c|c|c|c|c|c|c|c|c|c|c|c|c|}
\hline & VO & SI & IN & $\mathrm{CO}$ & $\mathrm{VC}$ & DSF & DSB & AR & BD & MR & VAL & SPR & $\mathrm{PC}$ \\
\hline VO & 1.00 & 0.62 & 0.69 & 0.61 & 0.67 & 0.20 & 0.20 & 0.46 & 0.37 & 0.32 & 0.30 & 0.30 & 0.23 \\
\hline SI & & 1.00 & 0.54 & 0.50 & 0.53 & 0.17 & 0.18 & 0.40 & 0.35 & 0.37 & 0.34 & 0.33 & 0.28 \\
\hline IN & & & 1.00 & 0.57 & 0.66 & 0.14 & 0.17 & 0.52 & 0.40 & 0.39 & 0.33 & 0.31 & 0.25 \\
\hline $\mathrm{CO}$ & & & & 1.00 & 0.46 & 0.13 & 0.16 & 0.39 & 0.31 & 0.28 & 0.27 & 0.22 & 0.27 \\
\hline $\mathrm{VC}$ & & & & & 1.00 & 0.19 & 0.25 & 0.51 & 0.48 & 0.39 & 0.35 & 0.42 & 0.31 \\
\hline DSF & & & & & & 1.00 & 0.47 & 0.27 & 0.16 & 0.15 & 0.23 & 0.24 & 0.13 \\
\hline DSB & & & & & & & 1.00 & 0.36 & 0.23 & 0.22 & 0.27 & 0.28 & 0.07 \\
\hline $\mathrm{AR}$ & & & & & & & & 1.00 & 0.51 & 0.42 & 0.40 & 0.41 & 0.31 \\
\hline BD & & & & & & & & & 1.00 & 0.51 & 0.39 & 0.56 & 0.41 \\
\hline MR & & & & & & & & & & 1.00 & 0.40 & 0.48 & 0.37 \\
\hline VAL & & & & & & & & & & & 1.00 & 0.42 & 0.30 \\
\hline SPR & & & & & & & & & & & & 1.00 & 0.35 \\
\hline $\mathrm{PC}$ & & & & & & & & & & & & & 1.00 \\
\hline
\end{tabular}


Table 52

Validation Dataset Correlation Matrix for Cognitive and Achievement Variables, continued

\begin{tabular}{|c|c|c|c|c|c|c|c|c|c|c|c|c|c|}
\hline & AS & tPLN & $\mathrm{CF}$ & SB & IW & LN & NR & MfW & $\mathrm{CD}$ & $\mathrm{SS}$ & $\mathrm{VM}$ & $\mathrm{RF}$ & DES \\
\hline VO & 0.36 & 0.25 & 0.41 & 0.45 & 0.34 & 0.36 & 0.24 & 0.29 & 0.14 & 0.23 & 0.20 & 0.27 & 0.17 \\
\hline SI & 0.34 & 0.21 & 0.40 & 0.34 & 0.27 & 0.32 & 0.21 & 0.25 & 0.18 & 0.20 & 0.19 & 0.29 & 0.19 \\
\hline $\mathrm{IN}$ & 0.39 & 0.28 & 0.40 & 0.34 & 0.25 & 0.35 & 0.20 & 0.24 & 0.03 & 0.15 & 0.11 & 0.16 & 0.08 \\
\hline $\mathrm{CO}$ & 0.32 & 0.23 & 0.31 & 0.30 & 0.19 & 0.30 & 0.17 & 0.21 & 0.11 & 0.16 & 0.12 & 0.25 & 0.17 \\
\hline $\mathrm{VC}$ & 0.46 & 0.27 & 0.51 & 0.44 & 0.38 & 0.36 & 0.27 & 0.27 & 0.10 & 0.16 & 0.18 & 0.16 & 0.23 \\
\hline DSF & 0.19 & 0.16 & 0.19 & 0.32 & 0.17 & 0.48 & 0.48 & 0.52 & 0.13 & 0.15 & 0.23 & 0.09 & 0.12 \\
\hline DSB & 0.35 & 0.21 & 0.30 & 0.20 & 0.11 & 0.48 & 0.64 & 0.38 & 0.18 & 0.17 & 0.30 & 0.10 & 0.17 \\
\hline $\mathrm{AR}$ & 0.48 & 0.33 & 0.48 & 0.27 & 0.31 & 0.50 & 0.38 & 0.27 & 0.18 & 0.21 & 0.31 & 0.16 & 0.19 \\
\hline $\mathrm{BD}$ & 0.46 & 0.39 & 0.46 & 0.24 & 0.23 & 0.31 & 0.23 & 0.22 & 0.25 & 0.37 & 0.34 & 0.16 & 0.33 \\
\hline MR & 0.40 & 0.32 & 0.41 & 0.24 & 0.21 & 0.35 & 0.22 & 0.17 & 0.27 & 0.26 & 0.26 & 0.20 & 0.21 \\
\hline VAL & 0.34 & 0.26 & 0.41 & 0.33 & 0.23 & 0.41 & 0.30 & 0.19 & 0.23 & 0.23 & 0.27 & 0.18 & 0.21 \\
\hline SPR & 0.38 & 0.26 & 0.44 & 0.25 & 0.22 & 0.36 & 0.30 & 0.24 & 0.21 & 0.26 & 0.32 & 0.13 & 0.23 \\
\hline $\mathrm{PC}$ & 0.25 & 0.30 & 0.37 & 0.21 & 0.18 & 0.26 & 0.06 & 0.20 & 0.22 & 0.26 & 0.22 & 0.15 & 0.27 \\
\hline \multicolumn{14}{|c|}{$\begin{array}{l}\text { Note. } \mathrm{VO}=\text { Vocabulary; SI = Similarities; IN = Information; CO = Comprehension; VC = Verbal Comprehension; DSF } \\
=\text { Digit Span - Forward; DSB = Digit Span }- \text { Backward; AR = Arithmetic; BD = Block Design; MR = Matrix } \\
\text { Reasoning; VAL = Visual-Auditory Learning; SPR = Spatial Relations; PC = Picture Completion; AS = Analysis- } \\
\text { Synthesis; tPLN = Planning with } 4^{\text {th }} \text {-Root Transformation; CF = Concept Formation; SB = Sound Blending; IW = } \\
\text { Incomplete Words; LNS = Letter-Number Sequencing; MfW = Memory for Words; CD = Coding; SS = Symbol Search; } \\
\text { VM = Visual Matching; RF = Retrieval Fluency; DES = Decision Speed. }\end{array}$} \\
\hline
\end{tabular}


Table 52

Validation Dataset Correlation Matrix for Cognitive and Achievement Variables, continued

\begin{tabular}{|c|c|c|c|c|c|c|c|c|c|c|c|c|c|}
\hline & RPN & $\mathrm{PrC}$ & PR & LWI & RFL & CAL & MFL & SP & WFL & PSC & AP & WS & WA \\
\hline VO & 0.19 & 0.14 & 0.13 & 0.60 & 0.50 & 0.35 & 0.21 & 0.54 & 0.38 & 0.61 & 0.42 & 0.42 & 0.30 \\
\hline SI & 0.13 & 0.19 & 0.19 & 0.40 & 0.36 & 0.32 & 0.16 & 0.30 & 0.33 & 0.48 & 0.36 & 0.40 & 0.16 \\
\hline $\mathrm{IN}$ & 0.02 & 0.10 & 0.15 & 0.54 & 0.32 & 0.51 & 0.18 & 0.45 & 0.21 & 0.58 & 0.52 & 0.39 & 0.23 \\
\hline $\mathrm{CO}$ & 0.10 & 0.13 & 0.18 & 0.34 & 0.30 & 0.32 & 0.18 & 0.19 & 0.28 & 0.44 & 0.38 & 0.35 & 0.11 \\
\hline $\mathrm{VC}$ & 0.18 & 0.12 & 0.20 & 0.63 & 0.44 & 0.49 & 0.20 & 0.56 & 0.35 & 0.68 & 0.58 & 0.48 & 0.34 \\
\hline DSF & 0.17 & 0.14 & 0.14 & 0.30 & 0.28 & 0.17 & 0.18 & 0.32 & 0.21 & 0.29 & 0.23 & 0.21 & 0.30 \\
\hline DSB & 0.12 & 0.17 & 0.17 & 0.30 & 0.24 & 0.32 & 0.27 & 0.30 & 0.21 & 0.35 & 0.37 & 0.29 & 0.30 \\
\hline AR & 0.11 & 0.19 & 0.18 & 0.40 & 0.31 & 0.61 & 0.43 & 0.39 & 0.25 & 0.49 & 0.70 & 0.37 & 0.31 \\
\hline BD & 0.13 & 0.35 & 0.27 & 0.28 & 0.29 & 0.48 & 0.25 & 0.24 & 0.29 & 0.43 & 0.54 & 0.35 & 0.21 \\
\hline MR & 0.12 & 0.23 & 0.20 & 0.28 & 0.21 & 0.42 & 0.20 & 0.24 & 0.21 & 0.36 & 0.46 & 0.30 & 0.18 \\
\hline VAL & 0.15 & 0.26 & 0.40 & 0.24 & 0.18 & 0.34 & 0.17 & 0.24 & 0.19 & 0.36 & 0.40 & 0.26 & 0.15 \\
\hline SPR & 0.09 & 0.26 & 0.23 & 0.27 & 0.23 & 0.40 & 0.16 & 0.21 & 0.21 & 0.39 & 0.45 & 0.32 & 0.22 \\
\hline $\mathrm{PC}$ & 0.17 & 0.23 & 0.24 & 0.15 & 0.18 & 0.20 & 0.06 & 0.11 & 0.16 & 0.28 & 0.25 & 0.23 & 0.06 \\
\hline
\end{tabular}


Table 52

Validation Dataset Correlation Matrix for Cognitive and Achievement Variables, continued

\begin{tabular}{|c|c|c|c|c|c|c|c|c|c|c|c|c|c|}
\hline & AS & $\mathrm{tPLN}$ & $\mathrm{CF}$ & SB & IW & LN & NR & MfW & $\mathrm{CD}$ & $\mathrm{SS}$ & VM & $\mathrm{RF}$ & DES \\
\hline AS & 1.00 & 0.35 & 0.50 & 0.25 & 0.20 & 0.34 & 0.30 & 0.18 & 0.20 & 0.16 & 0.28 & 0.09 & 0.19 \\
\hline tPLN & & 1.00 & 0.30 & 0.25 & 0.12 & 0.19 & 0.19 & 0.13 & 0.16 & 0.09 & 0.16 & 0.03 & 0.08 \\
\hline $\mathrm{CF}$ & & & 1.00 & 0.28 & 0.21 & 0.30 & 0.30 & 0.22 & 0.23 & 0.26 & 0.26 & 0.16 & 0.31 \\
\hline SB & & & & 1.00 & 0.48 & 0.34 & 0.23 & 0.35 & 0.09 & 0.17 & 0.13 & 0.17 & 0.14 \\
\hline IW & & & & & 1.00 & 0.30 & 0.22 & 0.20 & 0.08 & 0.18 & 0.19 & 0.24 & 0.13 \\
\hline LN & & & & & & 1.00 & 0.53 & 0.42 & 0.21 & 0.24 & 0.34 & 0.23 & 0.24 \\
\hline NR & & & & & & & 1.00 & 0.43 & 0.15 & 0.16 & 0.34 & 0.14 & 0.15 \\
\hline MfW & & & & & & & & 1.00 & 0.10 & 0.14 & 0.16 & 0.13 & 0.16 \\
\hline $\mathrm{CD}$ & & & & & & & & & 1.00 & 0.53 & 0.50 & 0.30 & 0.48 \\
\hline SS & & & & & & & & & & 1.00 & 0.56 & 0.35 & 0.54 \\
\hline VM & & & & & & & & & & & 1.00 & 0.37 & 0.59 \\
\hline $\mathrm{RF}$ & & & & & & & & & & & & 1.00 & 0.41 \\
\hline DES & & & & & & & & & & & & & 1.00 \\
\hline
\end{tabular}


Table 52

Validation Dataset Correlation Matrix for Cognitive and Achievement Variables, continued

\begin{tabular}{|c|c|c|c|c|c|c|c|c|c|c|c|c|c|}
\hline & RPN & $\operatorname{PrC}$ & PR & LWI & RFL & CAL & MFL & SP & WFL & PSC & AP & WS & WA \\
\hline AS & 0.04 & 0.15 & 0.19 & 0.34 & 0.25 & 0.54 & 0.25 & 0.34 & 0.18 & 0.42 & 0.57 & 0.37 & 0.23 \\
\hline tPLN & 0.02 & 0.10 & 0.20 & 0.23 & 0.15 & 0.37 & 0.11 & 0.13 & 0.09 & 0.28 & 0.37 & 0.26 & 0.11 \\
\hline $\mathrm{CF}$ & 0.12 & 0.17 & 0.24 & 0.35 & 0.28 & 0.45 & 0.20 & 0.33 & 0.24 & 0.49 & 0.54 & 0.40 & 0.27 \\
\hline SB & 0.19 & 0.10 & 0.21 & 0.43 & 0.31 & 0.18 & 0.13 & 0.36 & 0.35 & 0.43 & 0.27 & 0.33 & 0.33 \\
\hline IW & 0.30 & 0.12 & 0.18 & 0.33 & 0.32 & 0.12 & 0.11 & 0.28 & 0.32 & 0.31 & 0.22 & 0.25 & 0.19 \\
\hline $\mathrm{LN}$ & 0.23 & 0.22 & 0.28 & 0.40 & 0.37 & 0.37 & 0.27 & 0.33 & 0.32 & 0.40 & 0.41 & 0.28 & 0.33 \\
\hline NR & 0.18 & 0.20 & 0.14 & 0.32 & 0.28 & 0.29 & 0.29 & 0.37 & 0.22 & 0.32 & 0.36 & 0.30 & 0.37 \\
\hline MfW & 0.19 & 0.18 & 0.14 & 0.32 & 0.31 & 0.17 & 0.10 & 0.32 & 0.27 & 0.31 & 0.22 & 0.21 & 0.31 \\
\hline $\mathrm{CD}$ & 0.37 & 0.40 & 0.29 & 0.10 & 0.41 & 0.14 & 0.38 & 0.08 & 0.38 & 0.09 & 0.17 & 0.12 & 0.03 \\
\hline SS & 0.37 & 0.49 & 0.28 & 0.11 & 0.44 & 0.11 & 0.36 & 0.09 & 0.38 & 0.17 & 0.14 & 0.20 & 0.07 \\
\hline VM & 0.38 & 0.50 & 0.30 & 0.20 & 0.47 & 0.17 & 0.47 & 0.27 & 0.42 & 0.22 & 0.23 & 0.15 & 0.16 \\
\hline $\mathrm{RF}$ & 0.49 & 0.32 & 0.22 & 0.13 & 0.39 & -0.01 & 0.20 & 0.08 & 0.34 & 0.15 & 0.04 & 0.15 & -0.02 \\
\hline DES & 0.44 & 0.51 & 0.38 & 0.13 & 0.44 & 0.07 & 0.29 & 0.10 & 0.39 & 0.17 & 0.14 & 0.15 & 0.09 \\
\hline \multicolumn{14}{|c|}{$\begin{array}{l}\text { Note. } \text { AS = Analysis-Synthesis; tPLN = Planning with } 4^{\text {th }} \text {-Root Transformation; CF = Concept Formation; } \mathrm{SB}=\mathrm{Sound} \\
\text { Blending; IW = Incomplete Words; LNS = Letter-Number Sequencing; MfW = Memory for Words; CD = Coding; SS = } \\
\text { Symbol Search; VM = Visual Matching; RF = Retrieval Fluency; DES = Decision Speed; RPN = Rapid Picture Naming; } \\
\text { PrC = Pair Cancellation PR = Picture Recognition; LWI = Letter-Word Identification; RFL = Reading Fluency; CAL = } \\
\text { Calculation; MFL = Math Fluency; SP = Spelling; WFL = Writing Fluency; PSC = Passage Comprehension; AP = } \\
\text { Applied Problems; WS = Writing Samples; WA = Word Attack }\end{array}$} \\
\hline
\end{tabular}


Table 52

Validation Dataset Correlation Matrix for Cognitive and Achievement Variables, continued

\begin{tabular}{|c|c|c|c|c|c|c|c|c|c|c|c|c|c|}
\hline & RPN & $\operatorname{PrC}$ & PR & LWI & RFL & CAL & MFL & SP & WFL & PSC & AP & WS & WA \\
\hline RPN & 1.00 & 0.35 & 0.29 & 0.13 & 0.45 & -0.05 & 0.29 & 0.11 & 0.33 & 0.16 & 0.02 & 0.08 & 0.00 \\
\hline $\operatorname{PrC}$ & & 1.00 & 0.32 & 0.06 & 0.34 & 0.11 & 0.29 & 0.05 & 0.31 & 0.15 & 0.13 & 0.09 & 0.06 \\
\hline PR & & & 1.00 & 0.17 & 0.27 & 0.07 & 0.12 & 0.09 & 0.15 & 0.26 & 0.13 & 0.17 & 0.08 \\
\hline LWI & & & & 1.00 & 0.47 & 0.39 & 0.25 & 0.72 & 0.38 & 0.57 & 0.42 & 0.44 & 0.57 \\
\hline RFL & & & & & 1.00 & 0.20 & 0.49 & 0.46 & 0.58 & 0.42 & 0.27 & 0.35 & 0.29 \\
\hline CAL & & & & & & 1.00 & 0.43 & 0.39 & 0.15 & 0.41 & 0.78 & 0.39 & 0.32 \\
\hline MFL & & & & & & & 1.00 & 0.31 & 0.34 & 0.19 & 0.41 & 0.20 & 0.27 \\
\hline SP & & & & & & & & 1.00 & 0.36 & 0.52 & 0.41 & 0.46 & 0.56 \\
\hline WFL & & & & & & & & & 1.00 & 0.32 & 0.21 & 0.30 & 0.24 \\
\hline PSC & & & & & & & & & & 1.00 & 0.53 & 0.51 & 0.35 \\
\hline AP & & & & & & & & & & & 1.00 & 0.41 & 0.36 \\
\hline WS & & & & & & & & & & & & 1.00 & 0.35 \\
\hline WA & & & & & & & & & & & & & 1.00 \\
\hline
\end{tabular}


Table 53

Full Combined Dataset Correlation Matrix for Cognitive, Achievement, and Mental Health Variables

\begin{tabular}{|c|c|c|c|c|c|c|c|c|c|c|c|c|c|}
\hline & VO & SI & IN & $\mathrm{CO}$ & $\mathrm{VC}$ & DSF & DSB & AR & $\mathrm{BD}$ & MR & VAL & SPR & PC \\
\hline $\mathrm{VO}$ & 1.00 & 0.61 & 0.67 & 0.61 & 0.66 & 0.20 & 0.15 & 0.43 & 0.40 & 0.35 & 0.29 & 0.34 & 0.27 \\
\hline SI & & 1.00 & 0.52 & 0.48 & 0.52 & 0.19 & 0.15 & 0.39 & 0.36 & 0.38 & 0.32 & 0.34 & 0.32 \\
\hline IN & & & 1.00 & 0.52 & 0.63 & 0.15 & 0.14 & 0.50 & 0.41 & 0.35 & 0.32 & 0.34 & 0.23 \\
\hline $\mathrm{CO}$ & & & & 1.00 & 0.46 & 0.13 & 0.13 & 0.36 & 0.30 & 0.30 & 0.25 & 0.26 & 0.31 \\
\hline $\mathrm{VC}$ & & & & & 1.00 & 0.19 & 0.22 & 0.48 & 0.49 & 0.39 & 0.38 & 0.45 & 0.33 \\
\hline DSF & & & & & & 1.00 & 0.48 & 0.25 & 0.19 & 0.22 & 0.22 & 0.21 & 0.13 \\
\hline DSB & & & & & & & 1.00 & 0.31 & 0.21 & 0.26 & 0.26 & 0.25 & 0.10 \\
\hline $\mathrm{AR}$ & & & & & & & & 1.00 & 0.52 & 0.40 & 0.35 & 0.39 & 0.27 \\
\hline $\mathrm{BD}$ & & & & & & & & & 1.00 & 0.50 & 0.39 & 0.57 & 0.41 \\
\hline MR & & & & & & & & & & 1.00 & 0.39 & 0.51 & 0.38 \\
\hline VAL & & & & & & & & & & & 1.00 & 0.43 & 0.32 \\
\hline SPR & & & & & & & & & & & & 1.00 & 0.37 \\
\hline $\mathrm{PC}$ & & & & & & & & & & & & & 1.00 \\
\hline
\end{tabular}


Table 53

Full Combined Dataset Correlation Matrix for Cognitive, Achievement, and Mental Health Variables, continued

\begin{tabular}{|c|c|c|c|c|c|c|c|c|c|c|c|c|c|}
\hline & AS & tPLN & $\mathrm{CF}$ & SB & IW & LN & NR & MfW & $\mathrm{CD}$ & SS & VM & $\mathrm{RF}$ & DES \\
\hline VO & 0.34 & 0.28 & 0.39 & 0.43 & 0.33 & 0.30 & 0.20 & 0.25 & 0.14 & 0.23 & 0.17 & 0.27 & 0.12 \\
\hline SI & 0.30 & 0.24 & 0.37 & 0.38 & 0.30 & 0.29 & 0.18 & 0.22 & 0.16 & 0.20 & 0.17 & 0.28 & 0.16 \\
\hline IN & 0.38 & 0.29 & 0.37 & 0.32 & 0.21 & 0.31 & 0.20 & 0.23 & 0.05 & 0.16 & 0.12 & 0.15 & 0.07 \\
\hline $\mathrm{CO}$ & 0.31 & 0.23 & 0.31 & 0.32 & 0.22 & 0.25 & 0.14 & 0.18 & 0.10 & 0.16 & 0.13 & 0.26 & 0.14 \\
\hline $\mathrm{VC}$ & 0.44 & 0.29 & 0.50 & 0.46 & 0.36 & 0.35 & 0.27 & 0.26 & 0.12 & 0.20 & 0.19 & 0.20 & 0.20 \\
\hline DSF & 0.15 & 0.14 & 0.17 & 0.31 & 0.18 & 0.51 & 0.48 & 0.52 & 0.11 & 0.15 & 0.19 & 0.09 & 0.05 \\
\hline DSB & 0.30 & 0.21 & 0.27 & 0.22 & 0.11 & 0.48 & 0.61 & 0.39 & 0.20 & 0.15 & 0.28 & 0.08 & 0.14 \\
\hline $\mathrm{AR}$ & 0.43 & 0.31 & 0.45 & 0.27 & 0.24 & 0.40 & 0.34 & 0.25 & 0.15 & 0.20 & 0.25 & 0.15 & 0.12 \\
\hline $\mathrm{BD}$ & 0.44 & 0.40 & 0.46 & 0.28 & 0.25 & 0.34 & 0.26 & 0.24 & 0.23 & 0.36 & 0.35 & 0.17 & 0.28 \\
\hline MR & 0.39 & 0.35 & 0.42 & 0.28 & 0.24 & 0.36 & 0.27 & 0.21 & 0.19 & 0.26 & 0.25 & 0.19 & 0.18 \\
\hline VAL & 0.35 & 0.31 & 0.40 & 0.31 & 0.25 & 0.37 & 0.30 & 0.18 & 0.20 & 0.22 & 0.26 & 0.19 & 0.20 \\
\hline SPR & 0.39 & 0.35 & 0.46 & 0.32 & 0.26 & 0.33 & 0.29 & 0.24 & 0.16 & 0.25 & 0.28 & 0.13 & 0.20 \\
\hline $\mathrm{PC}$ & 0.23 & 0.30 & 0.36 & 0.24 & 0.19 & 0.26 & 0.07 & 0.18 & 0.23 & 0.26 & 0.22 & 0.18 & 0.26 \\
\hline \multicolumn{14}{|c|}{$\begin{array}{l}\text { Note. } \mathrm{VO}=\text { Vocabulary; SI = Similarities; IN = Information; CO = Comprehension; VC = Verbal Comprehension; DSF } \\
=\text { Digit Span - Forward; DSB = Digit Span - Backward; AR = Arithmetic; BD = Block Design; MR = Matrix } \\
\text { Reasoning; VAL = Visual-Auditory Learning; SPR = Spatial Relations; PC = Picture Completion; AS = Analysis- } \\
\text { Synthesis; tPLN = Planning with } 4^{\text {th }} \text {-Root Transformation; CF = Concept Formation; SB = Sound Blending; IW = } \\
\text { Incomplete Words; LNS = Letter-Number Sequencing; MfW = Memory for Words; CD = Coding; SS = Symbol Search; } \\
\text { VM = Visual Matching; RF = Retrieval Fluency; DES = Decision Speed. }\end{array}$} \\
\hline
\end{tabular}


Table 53

Full Combined Dataset Correlation Matrix for Cognitive, Achievement, and Mental Health Variables, continued

\begin{tabular}{|c|c|c|c|c|c|c|c|c|c|c|c|c|c|}
\hline & RPN & PrC & PR & LWI & RFL & CAL & MFL & SP & WFL & PSC & AP & WS & WA \\
\hline VO & 0.17 & 0.12 & 0.17 & 0.56 & 0.43 & 0.32 & 0.13 & 0.50 & 0.35 & 0.59 & 0.41 & 0.44 & 0.28 \\
\hline SI & 0.14 & 0.15 & 0.15 & 0.40 & 0.31 & 0.27 & 0.12 & 0.32 & 0.28 & 0.48 & 0.35 & 0.40 & 0.20 \\
\hline IN & 0.05 & 0.09 & 0.15 & 0.48 & 0.27 & 0.48 & 0.13 & 0.42 & 0.21 & 0.55 & 0.53 & 0.37 & 0.20 \\
\hline $\mathrm{CO}$ & 0.10 & 0.08 & 0.17 & 0.31 & 0.27 & 0.27 & 0.13 & 0.19 & 0.26 & 0.44 & 0.33 & 0.36 & 0.10 \\
\hline $\mathrm{VC}$ & 0.18 & 0.09 & 0.21 & 0.63 & 0.38 & 0.44 & 0.15 & 0.54 & 0.32 & 0.66 & 0.56 & 0.49 & 0.35 \\
\hline DSF & 0.14 & 0.12 & 0.15 & 0.32 & 0.25 & 0.14 & 0.17 & 0.29 & 0.16 & 0.27 & 0.24 & 0.18 & 0.25 \\
\hline DSB & 0.13 & 0.17 & 0.17 & 0.30 & 0.20 & 0.28 & 0.27 & 0.26 & 0.18 & 0.26 & 0.34 & 0.24 & 0.26 \\
\hline $\mathrm{AR}$ & 0.09 & 0.12 & 0.13 & 0.38 & 0.23 & 0.58 & 0.39 & 0.35 & 0.22 & 0.46 & 0.67 & 0.37 & 0.25 \\
\hline $\mathrm{BD}$ & 0.12 & 0.31 & 0.25 & 0.31 & 0.21 & 0.44 & 0.19 & 0.24 & 0.23 & 0.42 & 0.54 & 0.34 & 0.19 \\
\hline MR & 0.15 & 0.21 & 0.22 & 0.28 & 0.19 & 0.40 & 0.15 & 0.23 & 0.18 & 0.36 & 0.44 & 0.33 & 0.17 \\
\hline VAL & 0.17 & 0.24 & 0.37 & 0.25 & 0.15 & 0.31 & 0.12 & 0.23 & 0.15 & 0.36 & 0.37 & 0.25 & 0.13 \\
\hline SPR & 0.08 & 0.20 & 0.24 & 0.29 & 0.15 & 0.39 & 0.11 & 0.23 & 0.19 & 0.40 & 0.44 & 0.30 & 0.22 \\
\hline $\mathrm{PC}$ & 0.18 & 0.20 & 0.27 & 0.17 & 0.15 & 0.19 & 0.03 & 0.12 & 0.16 & 0.29 & 0.25 & 0.28 & 0.09 \\
\hline \multicolumn{14}{|c|}{$\begin{array}{l}\text { Note. } \mathrm{VO}=\text { Vocabulary; SI = Similarities; IN = Information; CO = Comprehension; VC = Verbal Comprehension; DSF } \\
=\text { Digit Span - Forward; DSB = Digit Span - Backward; AR = Arithmetic; BD = Block Design; MR = Matrix } \\
\text { Reasoning; VAL = Visual-Auditory Learning; SPR = Spatial Relations; PC = Picture Completion; RPN = Rapid Picture } \\
\text { Naming; PrC = Pair Cancellation; PR = Picture Recognition; LWI = Letter-Word Identification; RFL = Reading Fluency; } \\
\text { CAL = Calculation; MFL = Math Fluency; SP = Spelling; WFL = Writing Fluency; PSC = Passage Comprehension; AP } \\
=\text { Applied Problems; WS = Writing Samples; WA = Word Attack. }\end{array}$} \\
\hline
\end{tabular}


Table 53

Full Combined Dataset Correlation Matrix for Cognitive, Achievement, and Mental Health Variables, continued

\begin{tabular}{|c|c|c|c|c|c|c|c|c|c|c|}
\hline & SCA & SCB & $\mathrm{SCC}$ & SCD & SCE & SCF & SCG & $\mathrm{SCH}$ & BDI & SCL \\
\hline VO & 0.19 & -0.05 & 0.07 & 0.15 & 0.11 & 0.00 & 0.08 & 0.11 & 0.02 & 0.05 \\
\hline SI & 0.15 & -0.01 & 0.06 & 0.08 & 0.10 & 0.05 & 0.10 & 0.09 & 0.00 & 0.06 \\
\hline $\mathrm{IN}$ & 0.19 & -0.09 & 0.05 & 0.20 & 0.16 & 0.03 & 0.13 & 0.09 & 0.02 & 0.05 \\
\hline $\mathrm{CO}$ & 0.08 & -0.08 & 0.01 & 0.10 & 0.06 & -0.02 & 0.03 & 0.04 & -0.02 & 0.02 \\
\hline $\mathrm{VC}$ & 0.22 & -0.02 & 0.08 & 0.17 & 0.19 & 0.06 & 0.15 & 0.14 & 0.04 & 0.07 \\
\hline DSF & 0.05 & -0.04 & 0.01 & 0.06 & 0.06 & -0.01 & 0.05 & 0.02 & 0.04 & 0.06 \\
\hline DSB & 0.05 & -0.02 & 0.01 & 0.10 & 0.05 & -0.03 & 0.03 & 0.04 & 0.06 & 0.11 \\
\hline $\mathrm{AR}$ & 0.16 & 0.00 & 0.06 & 0.07 & 0.20 & 0.10 & 0.20 & 0.09 & -0.03 & 0.02 \\
\hline $\mathrm{BD}$ & 0.07 & -0.02 & 0.00 & 0.03 & 0.10 & 0.03 & 0.09 & 0.04 & -0.01 & 0.02 \\
\hline MR & 0.07 & -0.06 & -0.02 & 0.02 & 0.07 & -0.01 & 0.05 & 0.01 & -0.03 & 0.03 \\
\hline VAL & 0.05 & -0.02 & 0.00 & 0.08 & 0.00 & -0.02 & 0.01 & 0.02 & 0.05 & 0.07 \\
\hline SPR & 0.07 & -0.04 & 0.01 & 0.04 & 0.05 & -0.02 & 0.04 & 0.05 & -0.01 & 0.05 \\
\hline $\mathrm{PC}$ & 0.02 & 0.00 & -0.01 & -0.01 & -0.01 & -0.01 & -0.01 & 0.00 & -0.02 & -0.02 \\
\hline
\end{tabular}

Note. $\mathrm{VO}=$ Vocabulary; SI = Similarities; IN = Information; $\mathrm{CO}=$ Comprehension; VC = Verbal Comprehension; $\mathrm{DSF}=$ Digit Span - Forward; DSB = Digit Span - Backward; AR = Arithmetic; BD = Block Design; MR = Matrix Reasoning; VAL = Visual-Auditory Learning; SPR = Spatial Relations; PC = Picture Completion; SCA = Self-rated Conners Adult ADHD Rating Scale (CAARS) Inattention/Memory Problems subscale; SCB = Self-rated CAARS Hyperactivity/ Restlessness subscale; SCC = Self-rated CAARS Impulsivity/Emotional Lability subscale; SCD = Self-rated CAARS Problems with Self-Concept subscale; SCE = Self-rated CAARS DSM-IV Inattentive Symptoms subscale; SCF = Self-rated CAARS DSM-IV Hyperactive-Impulsive Symptoms subscale; SCG = Self-rated CAARS DSM-IV ADHD Symptoms Total subscale; SCH = Self-rated CAARS ADHD Index subscale; BDI = Beck Depression Inventory - 2 total; SCL = Symptom Checklist-90-Revised (SCL-90-R) Global Severity Index. 
Table 53

Full Combined Dataset Correlation Matrix for Cognitive, Achievement, and Mental Health Variables, continued

\begin{tabular}{|c|c|c|c|c|c|c|c|c|c|c|c|c|c|}
\hline & AS & tPLN & $\mathrm{CF}$ & SB & IW & LN & NR & MfW & $\mathrm{CD}$ & SS & VM & $\mathrm{RF}$ & DES \\
\hline $\mathrm{AS}$ & 1.00 & 0.38 & 0.48 & 0.24 & 0.17 & 0.29 & 0.30 & 0.17 & 0.20 & 0.16 & 0.28 & 0.11 & 0.16 \\
\hline tPLN & & 1.00 & 0.36 & 0.24 & 0.15 & 0.20 & 0.23 & 0.17 & 0.14 & 0.10 & 0.18 & 0.06 & 0.07 \\
\hline $\mathrm{CF}$ & & & 1.00 & 0.30 & 0.21 & 0.32 & 0.29 & 0.22 & 0.21 & 0.25 & 0.28 & 0.16 & 0.27 \\
\hline SB & & & & 1.00 & 0.47 & 0.32 & 0.23 & 0.34 & 0.10 & 0.21 & 0.14 & 0.22 & 0.15 \\
\hline IW & & & & & 1.00 & 0.26 & 0.19 & 0.20 & 0.11 & 0.19 & 0.22 & 0.26 & 0.16 \\
\hline $\mathrm{LN}$ & & & & & & 1.00 & 0.52 & 0.46 & 0.22 & 0.26 & 0.30 & 0.18 & 0.19 \\
\hline NR & & & & & & & 1.00 & 0.46 & 0.16 & 0.17 & 0.30 & 0.10 & 0.11 \\
\hline MfW & & & & & & & & 1.00 & 0.11 & 0.17 & 0.18 & 0.14 & 0.12 \\
\hline $\mathrm{CD}$ & & & & & & & & & 1.00 & 0.51 & 0.51 & 0.31 & 0.47 \\
\hline SS & & & & & & & & & & 1.00 & 0.53 & 0.33 & 0.50 \\
\hline VM & & & & & & & & & & & 1.00 & 0.36 & 0.57 \\
\hline $\mathrm{RF}$ & & & & & & & & & & & & 1.00 & 0.38 \\
\hline DES & & & & & & & & & & & & & 1.00 \\
\hline $\begin{array}{l}\text { Note. } \\
=\text { Digi } \\
\text { Reaso } \\
\text { Synth } \\
\text { Incom } \\
\text { VM = }\end{array}$ & $\begin{array}{l}=\mathrm{Voc} \\
\text { an }-\mathrm{F} \\
\text {; VAI } \\
\text { tPLN } \\
\text { e Wor } \\
\text { ual M }\end{array}$ & $\begin{array}{l}\text { bulary; } \\
\text { rward; } \\
=\text { Visua } \\
=\text { Planni } \\
\text { s; LNS } \\
\text { ching; }\end{array}$ & $\begin{array}{l}=\text { Sim } \\
\text { SB = D } \\
\text { Audito } \\
\text { with } \\
\text { Letter- } \\
=\text { Re }\end{array}$ & $\begin{array}{l}\text { rities; } \\
\text { it Spar } \\
\text { Learn } \\
\text {-Root } \\
\text { amber }\end{array}$ & $\begin{array}{l}=\text { Info } \\
\text { Backv } \\
\text {; SPR } \\
\text { ansforn } \\
\text { quenci }\end{array}$ & $\begin{array}{l}\text { nation; } \\
\text { rd; AR } \\
\text { Spatial } \\
\text { tion; C } \\
\text {; MfW }\end{array}$ & $\begin{array}{l}\mathrm{O}=\mathrm{C} \\
=\text { Arith } \\
\text { Relatio } \\
=\text { Con } \\
=\text { Mem }\end{array}$ & $\begin{array}{l}\text { mprehe } \\
\text { etic; B } \\
\text { s; PC = } \\
\text { ept For } \\
\text { ry for }\end{array}$ & $\begin{array}{l}\text { on; V } \\
\text { Bloc } \\
\text { cture } \\
\text { tion; } \\
\text { ds; C }\end{array}$ & $\begin{array}{l}=\text { Vert } \\
\text { esign } \\
\text { npleti } \\
=\text { Sou } \\
=\text { Codi }\end{array}$ & $\begin{array}{l}\text { Comp } \\
\mathrm{AR}=\mathrm{N} \\
\mathrm{AS}= \\
\text { Blend } \\
; \mathrm{SS}=\end{array}$ & $\begin{array}{l}\text { nension } \\
\text { trix } \\
\text { nalysis } \\
\text {; IW } \\
\text { ymbol }\end{array}$ & $\operatorname{arch}$ \\
\hline
\end{tabular}


Table 53

Full Combined Dataset Correlation Matrix for Cognitive, Achievement, and Mental Health Variables, continued

\begin{tabular}{|c|c|c|c|c|c|c|c|c|c|c|c|c|c|}
\hline & RPN & $\operatorname{PrC}$ & PR & LWI & RFL & CAL & MFL & SP & WFL & PSC & AP & WS & WA \\
\hline AS & 0.06 & 0.12 & 0.16 & 0.32 & 0.21 & 0.54 & 0.24 & 0.32 & 0.18 & 0.43 & 0.54 & 0.36 & 0.20 \\
\hline tPLN & 0.08 & 0.09 & 0.16 & 0.20 & 0.13 & 0.35 & 0.08 & 0.15 & 0.08 & 0.31 & 0.38 & 0.31 & 0.11 \\
\hline $\mathrm{CF}$ & 0.11 & 0.15 & 0.26 & 0.36 & 0.24 & 0.41 & 0.15 & 0.31 & 0.25 & 0.47 & 0.50 & 0.39 & 0.26 \\
\hline SB & 0.20 & 0.09 & 0.24 & 0.43 & 0.26 & 0.17 & 0.08 & 0.33 & 0.31 & 0.42 & 0.24 & 0.35 & 0.29 \\
\hline IW & 0.27 & 0.13 & 0.21 & 0.28 & 0.28 & 0.09 & 0.09 & 0.24 & 0.24 & 0.30 & 0.19 & 0.23 & 0.19 \\
\hline LN & 0.20 & 0.21 & 0.26 & 0.38 & 0.32 & 0.33 & 0.25 & 0.31 & 0.27 & 0.37 & 0.37 & 0.25 & 0.25 \\
\hline NR & 0.14 & 0.17 & 0.20 & 0.33 & 0.27 & 0.27 & 0.28 & 0.33 & 0.19 & 0.29 & 0.37 & 0.26 & 0.31 \\
\hline MfW & 0.14 & 0.17 & 0.13 & 0.34 & 0.28 & 0.15 & 0.13 & 0.31 & 0.27 & 0.29 & 0.23 & 0.20 & 0.29 \\
\hline $\mathrm{CD}$ & 0.40 & 0.41 & 0.23 & 0.12 & 0.41 & 0.14 & 0.39 & 0.12 & 0.38 & 0.13 & 0.16 & 0.10 & 0.05 \\
\hline SS & 0.35 & 0.50 & 0.27 & 0.15 & 0.45 & 0.11 & 0.33 & 0.11 & 0.35 & 0.23 & 0.16 & 0.16 & 0.09 \\
\hline VM & 0.38 & 0.51 & 0.27 & 0.20 & 0.46 & 0.18 & 0.47 & 0.26 & 0.37 & 0.22 & 0.24 & 0.13 & 0.15 \\
\hline $\mathrm{RF}$ & 0.47 & 0.27 & 0.22 & 0.14 & 0.35 & 0.01 & 0.22 & 0.08 & 0.34 & 0.16 & 0.05 & 0.17 & -0.02 \\
\hline DES & 0.42 & 0.48 & 0.34 & 0.11 & 0.37 & 0.04 & 0.27 & 0.06 & 0.32 & 0.14 & 0.09 & 0.11 & 0.05 \\
\hline
\end{tabular}

Note. AS = Analysis-Synthesis; tPLN = Planning with $4^{\text {th }}$-Root Transformation; CF $=$ Concept Formation; SB = Sound Blending; IW = Incomplete Words; LNS = Letter-Number Sequencing; MfW = Memory for Words; CD = Coding; SS = Symbol Search; VM = Visual Matching; RF = Retrieval Fluency; DES = Decision Speed; RPN = Rapid Picture Naming; $\mathrm{PrC}=$ Pair Cancellation; $\mathrm{PR}=$ Picture Recognition; LWI = Letter-Word Identification; RFL = Reading Fluency; CAL = Calculation; MFL = Math Fluency; SP = Spelling; WFL = Writing Fluency; PSC = Passage Comprehension; AP = Applied Problems; WS = Writing Samples; WA = Word Attack 
Table 53

Full Combined Dataset Correlation Matrix for Cognitive, Achievement, and Mental Health Variables, continued

\begin{tabular}{lrrrrrrrrrr}
\hline & SCA & SCB & SCC & SCD & SCE & SCF & SCG & SCH & BDI & SCL \\
\hline AS & 0.19 & -0.05 & 0.07 & 0.15 & 0.11 & 0.00 & 0.08 & 0.11 & 0.02 & 0.05 \\
tPLN & 0.15 & -0.01 & 0.06 & 0.08 & 0.10 & 0.05 & 0.10 & 0.09 & 0.00 & 0.06 \\
CF & 0.19 & -0.09 & 0.05 & 0.20 & 0.16 & 0.03 & 0.13 & 0.09 & 0.02 & 0.05 \\
SB & 0.08 & -0.08 & 0.01 & 0.10 & 0.06 & -0.02 & 0.03 & 0.04 & -0.02 & 0.02 \\
IW & 0.22 & -0.02 & 0.08 & 0.17 & 0.19 & 0.06 & 0.15 & 0.14 & 0.04 & 0.07 \\
LN & 0.05 & -0.04 & 0.01 & 0.06 & 0.06 & -0.01 & 0.05 & 0.02 & 0.04 & 0.06 \\
NR & 0.05 & -0.02 & 0.01 & 0.10 & 0.05 & -0.03 & 0.03 & 0.04 & 0.06 & 0.11 \\
MfW & 0.16 & 0.00 & 0.06 & 0.07 & 0.20 & 0.10 & 0.20 & 0.09 & -0.03 & 0.02 \\
CD & 0.07 & -0.02 & 0.00 & 0.03 & 0.10 & 0.03 & 0.09 & 0.04 & -0.01 & 0.02 \\
SS & 0.07 & -0.06 & -0.02 & 0.02 & 0.07 & -0.01 & 0.05 & 0.01 & -0.03 & 0.03 \\
VM & 0.05 & -0.02 & 0.00 & 0.08 & 0.00 & -0.02 & 0.01 & 0.02 & 0.05 & 0.07 \\
RF & 0.07 & -0.04 & 0.01 & 0.04 & 0.05 & -0.02 & 0.04 & 0.05 & -0.01 & 0.05 \\
DES & 0.02 & 0.00 & -0.01 & -0.01 & -0.01 & -0.01 & -0.01 & 0.00 & -0.02 & -0.02
\end{tabular}

Note. AS $=$ Analysis-Synthesis; tPLN $=$ Planning with $4^{\text {th }}$-Root Transformation; $\mathrm{CF}=$ Concept Formation; $\mathrm{SB}=\mathrm{Sound}$ Blending; IW = Incomplete Words; LNS = Letter-Number Sequencing; MfW = Memory for Words; CD = Coding; SS = Symbol Search; VM = Visual Matching; RF = Retrieval Fluency; DES = Decision Speed; SCA = Self-rated Conners Adult ADHD Rating Scale (CAARS) Inattention/Memory Problems subscale; SCB = Self-rated CAARS Hyperactivity/ Restlessness subscale; SCC = Self-rated CAARS Impulsivity/Emotional Lability subscale; SCD = Self-rated CAARS Problems with Self-Concept subscale; SCE = Self-rated CAARS DSM-IV Inattentive Symptoms subscale; SCF $=$ Selfrated CAARS DSM-IV Hyperactive-Impulsive Symptoms subscale; SCG = Self-rated CAARS DSM-IV ADHD

Symptoms Total subscale; SCH = Self-rated CAARS ADHD Index subscale; BDI = Beck Depression Inventory -2 total; SCL = Symptom Checklist-90-Revised (SCL-90-R) Global Severity Index. 
Table 53

Full Combined Dataset Correlation Matrix for Cognitive, Achievement, and Mental Health Variables, continued

\begin{tabular}{|c|c|c|c|c|c|c|c|c|c|c|c|c|c|}
\hline & $\mathrm{RPN}$ & $\operatorname{PrC}$ & PR & LWI & RFL & CAL & MFL & SP & WFL & PSC & AP & WS & WA \\
\hline RPN & 1.00 & 0.32 & 0.26 & 0.13 & 0.45 & -0.04 & 0.28 & 0.10 & 0.30 & 0.18 & 0.02 & 0.08 & 0.01 \\
\hline $\operatorname{PrC}$ & & 1.00 & 0.24 & 0.06 & 0.31 & 0.06 & 0.30 & 0.06 & 0.27 & 0.14 & 0.09 & 0.04 & 0.05 \\
\hline PR & & & 1.00 & 0.15 & 0.20 & 0.07 & 0.09 & 0.08 & 0.14 & 0.23 & 0.11 & 0.17 & 0.06 \\
\hline LWI & & & & 1.00 & 0.40 & 0.33 & 0.21 & 0.70 & 0.37 & 0.54 & 0.43 & 0.44 & 0.57 \\
\hline RFL & & & & & 1.00 & 0.13 & 0.43 & 0.44 & 0.54 & 0.43 & 0.22 & 0.30 & 0.27 \\
\hline CAL & & & & & & 1.00 & 0.41 & 0.34 & 0.11 & 0.38 & 0.76 & 0.35 & 0.23 \\
\hline MFL & & & & & & & 1.00 & 0.30 & 0.33 & 0.18 & 0.40 & 0.15 & 0.22 \\
\hline SP & & & & & & & & 1.00 & 0.37 & 0.50 & 0.39 & 0.44 & 0.55 \\
\hline WFL & & & & & & & & & 1.00 & 0.30 & 0.18 & 0.25 & 0.24 \\
\hline PSC & & & & & & & & & & 1.00 & 0.49 & 0.48 & 0.37 \\
\hline AP & & & & & & & & & & & 1.00 & 0.40 & 0.31 \\
\hline WS & & & & & & & & & & & & 1.00 & 0.32 \\
\hline WA & & & & & & & & & & & & & 1.00 \\
\hline
\end{tabular}


Table 53

Full Combined Dataset Correlation Matrix for Cognitive, Achievement, and Mental Health Variables, continued

\begin{tabular}{|c|c|c|c|c|c|c|c|c|c|c|}
\hline & $\mathrm{SCA}$ & SCB & $\mathrm{SCC}$ & SCD & SCE & SCF & $\mathrm{SCG}$ & $\mathrm{SCH}$ & BDI & SCL \\
\hline RPN & -0.12 & -0.04 & -0.07 & -0.07 & -0.15 & -0.09 & -0.13 & -0.09 & -0.03 & -0.04 \\
\hline $\operatorname{PrC}$ & -0.07 & -0.05 & -0.02 & -0.03 & -0.10 & -0.03 & -0.07 & -0.05 & -0.03 & -0.03 \\
\hline PR & -0.03 & -0.08 & -0.05 & -0.01 & -0.09 & -0.11 & -0.11 & -0.05 & -0.01 & 0.02 \\
\hline LWI & 0.23 & -0.02 & 0.10 & 0.18 & 0.21 & 0.05 & 0.15 & 0.16 & 0.04 & 0.09 \\
\hline RFL & 0.06 & -0.02 & 0.04 & 0.03 & 0.02 & -0.02 & 0.01 & 0.06 & -0.01 & -0.05 \\
\hline CAL & 0.14 & -0.05 & 0.03 & 0.14 & 0.15 & 0.02 & 0.12 & 0.10 & 0.04 & 0.06 \\
\hline MFL & 0.01 & 0.01 & 0.02 & 0.02 & 0.04 & 0.02 & 0.04 & 0.03 & -0.01 & 0.00 \\
\hline SP & 0.25 & 0.00 & 0.10 & 0.20 & 0.18 & 0.01 & 0.12 & 0.16 & 0.10 & 0.09 \\
\hline WFL & 0.11 & 0.08 & 0.10 & 0.01 & 0.03 & 0.06 & 0.05 & 0.10 & -0.03 & -0.06 \\
\hline PSC & 0.18 & -0.06 & 0.02 & 0.11 & 0.14 & 0.00 & 0.10 & 0.09 & -0.02 & 0.04 \\
\hline AP & 0.14 & -0.04 & 0.02 & 0.13 & 0.20 & 0.06 & 0.18 & 0.10 & 0.04 & 0.08 \\
\hline WS & 0.13 & -0.05 & 0.00 & 0.07 & 0.08 & -0.02 & 0.05 & 0.05 & 0.00 & 0.01 \\
\hline WA & 0.19 & 0.05 & 0.05 & 0.08 & 0.20 & 0.05 & 0.15 & 0.12 & 0.00 & 0.04 \\
\hline
\end{tabular}

Note. RPN = Rapid Picture Naming; PrC = Pair Cancellation; PR = Picture Recognition; LWI = Letter-Word

Identification; RFL = Reading Fluency; $\mathrm{CAL}=$ Calculation; $\mathrm{MFL}=$ Math Fluency; $\mathrm{SP}=$ Spelling; WFL = Writing

Fluency; PSC = Passage Comprehension; AP = Applied Problems; WS = Writing Samples; WA = Word Attack; SCA =

Self-rated Conners Adult ADHD Rating Scale (CAARS) Inattention/Memory Problems subscale; SCB = Self-rated

CAARS Hyperactivity/ Restlessness subscale; SCC = Self-rated CAARS Impulsivity/Emotional Lability subscale; SCD

$=$ Self-rated CAARS Problems with Self-Concept subscale; SCE = Self-rated CAARS DSM-IV Inattentive Symptoms

subscale; SCF = Self-rated CAARS DSM-IV Hyperactive-Impulsive Symptoms subscale; SCG = Self-rated CAARS

DSM-IV ADHD Symptoms Total subscale; SCH = Self-rated CAARS ADHD Index subscale; BDI = Beck Depression

Inventory -2 total; SCL = Symptom Checklist-90-Revised (SCL-90-R) Global Severity Index. 
Table 53

Full Combined Dataset Correlation Matrix for Cognitive, Achievement, and Mental Health Variables, continued

\begin{tabular}{|c|c|c|c|c|c|c|c|c|c|c|}
\hline & SCA & SCB & SCC & SCD & SCE & $\mathrm{SCF}$ & SCG & $\mathrm{SCH}$ & BDI & SCL \\
\hline SCA & 1.00 & 0.45 & 0.61 & 0.54 & 0.83 & 0.48 & 0.72 & 0.78 & 0.30 & 0.31 \\
\hline SCB & & 1.00 & 0.59 & 0.19 & 0.48 & 0.80 & 0.69 & 0.65 & 0.16 & 0.21 \\
\hline $\mathrm{SCC}$ & & & 1.00 & 0.47 & 0.56 & 0.69 & 0.67 & 0.78 & 0.34 & 0.32 \\
\hline SCD & & & & 1.00 & 0.46 & 0.21 & 0.37 & 0.65 & 0.57 & 0.49 \\
\hline SCE & & & & & 1.00 & 0.58 & 0.89 & 0.73 & 0.27 & 0.34 \\
\hline $\mathrm{SCF}$ & & & & & & 1.00 & 0.85 & 0.66 & 0.15 & 0.23 \\
\hline $\mathrm{SCG}$ & & & & & & & 1.00 & 0.76 & 0.23 & 0.32 \\
\hline $\mathrm{SCH}$ & & & & & & & & 1.00 & 0.41 & 0.42 \\
\hline BDI & & & & & & & & & 1.00 & 0.64 \\
\hline SCL & & & & & & & & & & 1.00 \\
\hline
\end{tabular}

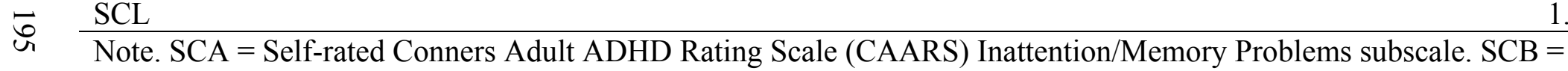
Self-rated CAARS Hyperactivity/ Restlessness subscale. SCC = Self-rated CAARS Impulsivity/Emotional Lability subscale. SCD = Self-rated CAARS Problems with Self-Concept subscale. SCE = Self-rated CAARS DSM-IV Inattentive Symptoms. SCF = Self-rated CAARS DSM-IV Hyperactive-Impulsive Symptoms. SCG = Self-rated CAARS DSM-IV ADHD Symptoms Total. SCH = Self-rated CAARS ADHD Index. BDI = Beck Depression Inventory -2. SCL = Symptom Checklist-90-Revised (SCL-90-R) Global Severity Index. 


\section{Model 1 - Hierarchical and Bifactor $g$ Models}

An initial confirmatory factor analysis of the calibration dataset tested the first CHC model hypothesized in Chapter II. The first model hypothesized a CHC-inspired structure in which $g$ forms the hierarchical apex over seven broad and nine narrow latent factors which are measured by 28 manifest indicators. The model as originally hypothesized provided a fair fit for the calibration data $(\mathrm{CFI}=.88, \mathrm{SRMR}=.068$, RMSEA $=.062)$. Because the calibration data did not fit the model as well as hoped, a parallel analysis was used to compare the obtained eigenvalues to eigenvalues that would be obtained from random data (Hayton, Allen \& Scarpello, 2004). The factor solution in a parallel analysis is generated by selecting the number of factors whose eigenvalues are greater than what would be expected from the random data. The parallel analysis of the correlation matrix indicated five factors, two fewer than in the hypothesized seven-factor structure of the WJ-III derived from the standardization sample (McGrew \& Woodcock, 2001).

Based on the parallel analysis results, exploratory analyses were conducted using the principal axis method for factor extraction, a method that focuses on common variance among the variables (Fabrigar, Wegener, MacCullum, \& Strahan, 1999). To allow measures to correlate with each other, the principal axis method was combined with promax oblique rotation method to interpret the results. (Fabrigar et al., 1999). Table 54 lists the item loadings for all administered subtests the WAIS-III and WAIS-IV have in common, as well as all administered WJ III cognitive subtests. For greater ease in reading the table and interpreting the results, loadings less than 0.20 were omitted. 
Table 54

Factor Loadings for WAIS-III/IV Combined Calibration Dataset

\begin{tabular}{|c|c|c|c|c|c|c|}
\hline Subtest & $\mathrm{G} c$ & $\mathrm{G} f / \mathrm{G} v$ & Gsm & $\mathrm{G} a$ & $\mathrm{Gs} / \mathrm{G} l r$ & Communality \\
\hline Vocabulary & 0.86 & & & & & 0.75 \\
\hline Similarities & 0.63 & & & & & 0.49 \\
\hline Information & 0.77 & & & & & 0.61 \\
\hline Comprehension & 0.62 & & & & & 0.46 \\
\hline Verbal Comprehension & 0.59 & 0.24 & & & & 0.63 \\
\hline Digit Span - Forward & & & 0.78 & & & 0.55 \\
\hline Digit Span - Backward & & & 0.68 & & & 0.50 \\
\hline Block Design & 0.25 & 0.47 & & & & 0.53 \\
\hline Matrix Reasoning & & 0.55 & & & & 0.45 \\
\hline Visual-Auditory Learning & & 0.55 & & & & 0.40 \\
\hline Spatial Relations & & 0.69 & & & & 0.52 \\
\hline Picture Completion & & 0.41 & & 0.20 & & 0.33 \\
\hline Analysis-Synthesis & & 0.51 & & & & 0.41 \\
\hline Planning & & 0.55 & & & & 0.31 \\
\hline Concept Formation & & 0.61 & & & & 0.47 \\
\hline Sound Blending & 0.27 & & 0.22 & 0.41 & & 0.49 \\
\hline Incomplete Words & & & & 0.43 & & 0.35 \\
\hline Letter-Number Sequencing & & & 0.64 & & & 0.53 \\
\hline Numbers Reversed & & 0.20 & 0.70 & & & 0.58 \\
\hline Memory for Words & & & 0.65 & & & 0.46 \\
\hline Coding & & & & & 0.68 & 0.45 \\
\hline Symbol Search & & & & & 0.64 & 0.45 \\
\hline Visual Matching & & & & & 0.73 & 0.60 \\
\hline Retrieval Fluency & 0.22 & & & 0.33 & 0.39 & 0.37 \\
\hline Decision Speed & & & & & 0.70 & 0.53 \\
\hline Rapid Picture Naming & & & & 0.31 & 0.47 & 0.37 \\
\hline Pair Cancellation & & & & & 0.69 & 0.45 \\
\hline Picture Recognition & & 0.34 & & 0.32 & & 0.28 \\
\hline
\end{tabular}

Note. Table based on a principal axis factor analysis with promax rotation. Loadings under 0.20 are suppressed. $\mathrm{G} c=$ Comprehension-Knowledge. $\mathrm{G} f=$ Fluid Reasoning. $\mathrm{G} v$ $=$ Visual-Spatial Processing. Gsm $=$ Working Memory Capacity. G $a=$ Auditory Processing. Gs = Processing Speed. Glr = Long-term Storage and Retrieval. 
Although an examination of exploratory factor loadings suggested some possible refinements to the originally hypothesized model, caution in interpreting the exploratory analysis is warranted (Bowden, 2013). Because variance that is attributable to the hypothesized model is not distinguished from variance that is attributable to measurement error in an exploratory analysis, sample-specific error can adversely influence results and may result in a model that is not generalizable (Henson \& Roberts, 2006). Therefore, only theoretically consistent loadings indicated by the exploratory analyses were inserted into the model. One interesting finding from the exploratory analysis was the lack of distinction between the fluid reasoning $(\mathrm{G} f)$ and visual-spatial processing $(\mathrm{G} v)$ subtests. All $\mathrm{G} f$ and $\mathrm{G} v$ subtests belonged to a common factor. Subsequent confirmatory analyses indicated, however, that these variables indeed loaded distinctly onto two (highly correlated) factors.

The exploratory analysis also indicated no information about the existence or placement of potential narrow factors. Because the narrow factors were hypothesized in the original model, exploratory analyses of each hypothesized broad factor were conducted to determine whether or where narrow factors were indicated. Parallel analyses of each broad factor suggested only one factor each for comprehensionknowledge $(\mathrm{Gc})$, fluid reasoning $(\mathrm{G} f)$, and processing speed $(\mathrm{Gs})$. Two possible factors emerged for visual-spatial processing (Gv) that included the Visual Memory (MV) subtests Picture Completion and Picture Recognition on one factor and all other subtests on another factor, presumably Visualization (Vz). Working-memory capacity (Gsm) also divided into two factors, including a working memory factor (MW) that included 
Numbers Reversed (NR), Digit Span - Backward (DSB), and Letter-Number Sequencing (LN), and a short-term memory factor (MS) that included Digit Span - Forward (DSF) and Memory for Words (MfW).

Because the exploratory analyses yielded no initial distinction between the fluid reasoning $(\mathrm{G} f)$ and visual-spatial processing $(\mathrm{G} v)$ items, calibration confirmatory analyses tested the narrow factors and possible cross-loadings suggested by the exploratory analyses, CHC theory (Schneider \& McGrew, 2012), and previous research (e.g., Benson et al., 2010; Weiss et al., 2013). Keeping CHC theory in mind and integrating theoretically consistent results of the exploratory analyses with the originally hypothesized model, the $g$ cognitive model was refined to improve model fit. Further, some recent studies have highlighted the utility of the Bifactor model in which $g$ has been examined as a breadth factor rather than as solely at the hierarchical apex over the primary factors (Golay, Reverte, Rossier, Favez, \& Lecerf, 2012; Reise, Moore, \& Haviland, 2010). Bifactor analysis can be useful in identifying the general factor, $g$, and group factors by exposing the specific remaining common item variance that is uncorrelated with the general factor. With each item loading on the general factor, the general factor reflects item commonality and individual differences with respect to each item's relation with the general factor. The other factors in the bifactor model are orthogonal to the general factor and represent what the items have in common through item variance that is not accounted for by the general factor. By estimating the relative extent of both the general and specific factors, one can infer a possible hierarchical structure of the data as well as a cleaner view of each group factor's relation to the 
general factor (Reise et al., 2010).

Similar to the findings of Golay and colleagues (2012) when they explored the French WISC-IV through Bayesian structural equation modeling, an examination of the first calibration analysis suggests that, for this dataset, loadings on the broad factors are not as clean as typically indicated (e.g., Weiss et al.2013). To extract the most variance possible, all significant loadings were allowed to load onto their respective factors if they could be justified by CHC theory and previous research. Several subtests loaded on multiple factors giving one indication that the interpretation of intelligence for this sample may not be as clear cut as originally hypothesized.

The final models of the hierarchical $g$ and bifactor $g$ models of the Full Combined dataset are shown in Figures 10 and 11; the fit statistics for these models from each of the datasets are described in Table 55. Once the model was identified with the calibration dataset, it was tested with the validation dataset. The validation dataset indicated a good fit for the data, as did the Full Combined dataset. As indicated in Table 55, the fit improved with each dataset. All three datasets indicated a good fit with the hierarchical model but a better fit with the bifactor model. 


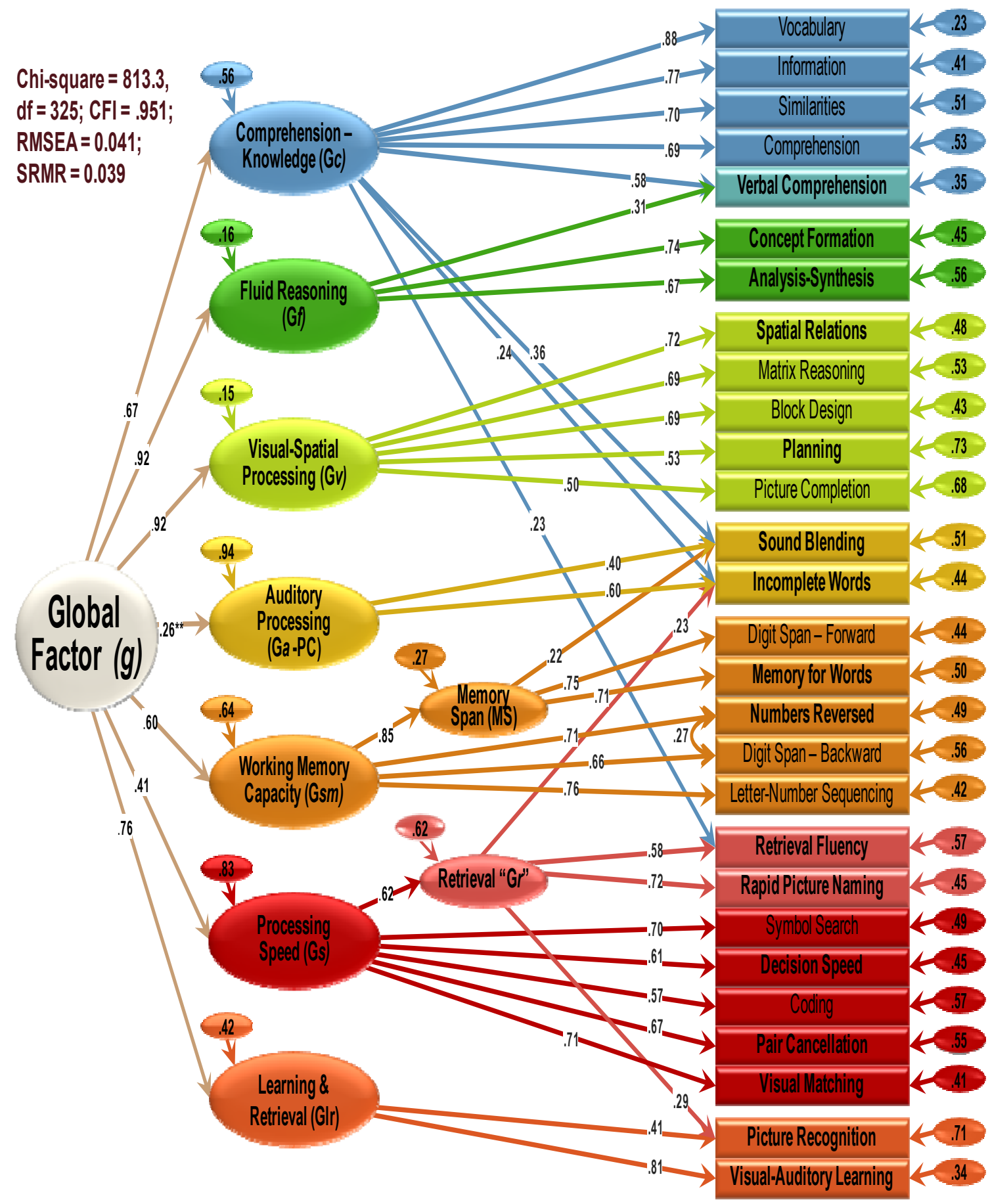

Figure 10. Final hierarchical $g$ model of WAIS-III/IV and WJ III COG with CHC broad and narrow factors. WJ III subtests are noted in bold. Completely standardized maximum likelihood parameter estimates. All estimates significant $p<.001$ except $* * p<.01$. 


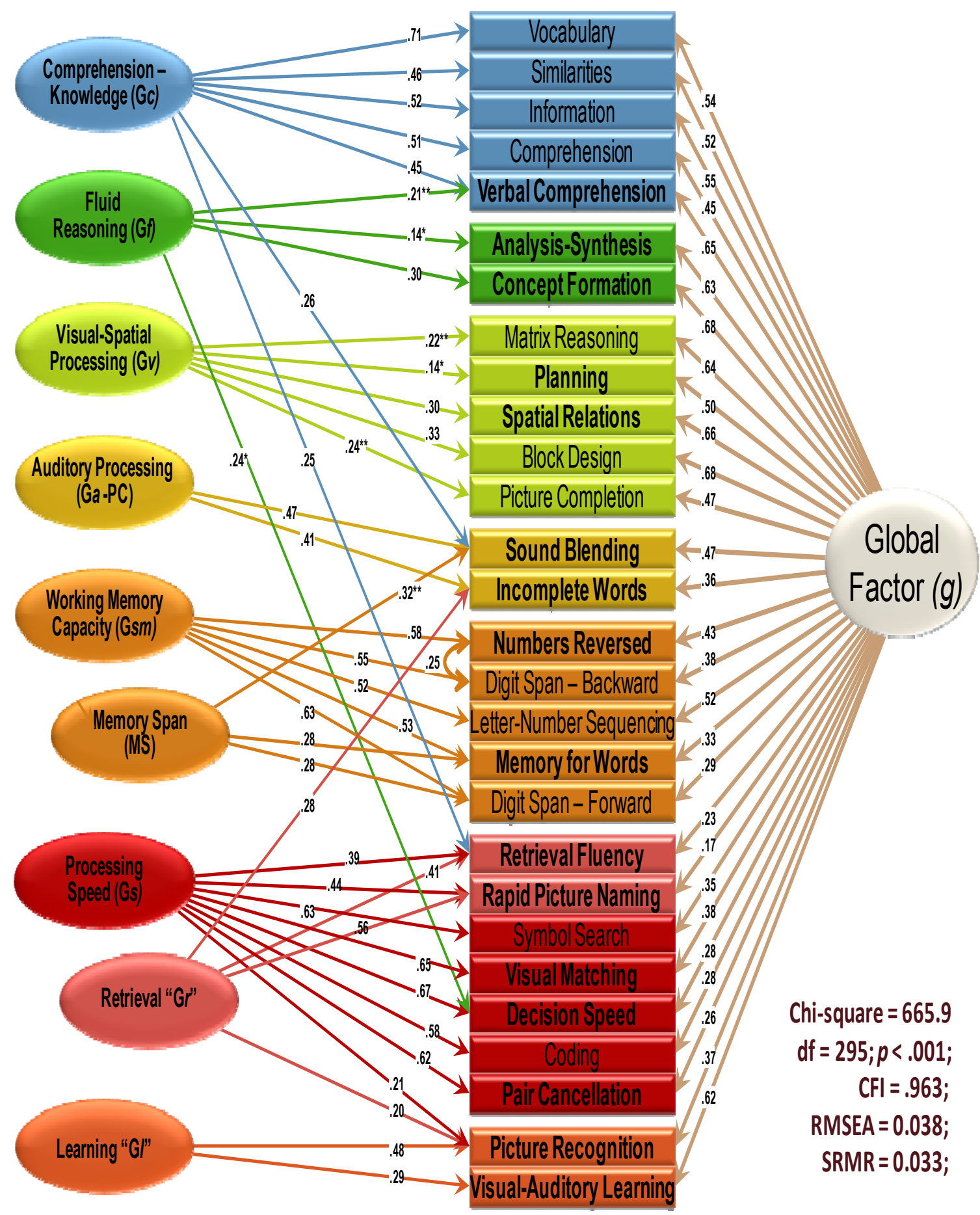

Figure 11. Final bifactor $g$ model of WAIS-III/IV and WJ III COG with CHC broad factors. WJ III subtests are noted in bold. Completely standardized maximum likelihood parameter estimates. All estimates significant $p<.001$ except ${ }^{* *} p<.01$. 
The factor loadings on the hierarchical $g$ and bifactor models were similar but not identical. Differences included a small cross-loading of Decision Speed on G $f$ on the bifactor model that was not indicated on the hierarchical model. Further, although all latent factors in the bifactor model that contained only two parameters had their parameters constrained to be equal, none of these groups required such constraints on the hierarchical model. The bifactor model is a "flat" model; that is, there are no narrow factors under the broad factors. Therefore, narrow factors were represented as separate entities under the bifactor model, but the subtests that loaded on them also loaded on the broad factor that subsumed the narrow factor in the hierarchical model. The structure of the two models was made as similar as possible to compare fit quality between the hierarchical and bifactor models. As indicated in Table 55, the bifactor model provides a better fit for the data than does the hierarchical model.

Table 55

Hypotheses Testing $-g$ Hierarchical and Bifactor Models

\begin{tabular}{lccccccccc}
\hline Model/Sample & $n$ & $\chi^{2}$ & $d f$ & $\Delta \chi^{2 \mathrm{a}}$ & $\Delta d f^{\mathrm{a}}$ & $p$ & CFI & RMSEA & SRMR \\
\hline $\begin{array}{l}\text { Hierarchical } g \\
\quad \text { Calibration }\end{array}$ & 431 & 540.11 & 325 & & & & .954 & .039 & .042 \\
$\begin{array}{c}\text { Bifactor } g \\
\quad \text { Calibration }\end{array}$ & 431 & 475.24 & 295 & 64.87 & 30 & $<.001$ & .961 & .038 & .037 \\
$\begin{array}{c}\text { Hierarchical } g \\
\quad \text { Validation }\end{array}$ & 458 & 570.50 & 325 & & & & .953 & .041 & .046 \\
$\begin{array}{c}\text { Bifactor } g \\
\quad \text { Validation }\end{array}$ & 458 & 461.44 & 295 & 109.06 & 30 & $<.001$ & .968 & .035 & .039 \\
$\begin{array}{c}\text { Hierarchical } g \\
\quad \text { Full Combined }\end{array}$ & 889 & 813.26 & 325 & & & & .951 & .041 & .039 \\
$\begin{array}{c}\text { Bifactor } g \\
\quad \text { Full Combined }\end{array}$ & 889 & 665.85 & 295 & 147.41 & 30 & $<.001$ & .963 & .038 & .033 \\
\hline
\end{tabular}

Note. ${ }^{\mathrm{a}}$ Compared to previous model. CFI $=$ Comparative Fit Index. RMSEA $=$ Root Mean Square Error of Approximation. SRMR $=$ Standardized Root Mean Square Residual. 
Among the additions made to Model 1 to improve fit included several small but significant cross-loadings of the auditory processing $(\mathrm{G} a)$ subtest Sound Blending. These additional loadings make theoretical sense when recognizing all that is required to perform the task Sound Blending requires, namely, to recognize discrete speech sounds as words. For example, Sound Blending's cross-loading on comprehension-knowledge $(\mathrm{Gc})$ makes sense when realizing that a person is more likely to recognize particular words from discrete speech sounds if one knows more words. Another refinement made to Model 1 included the removal of the narrow factors that were not supported by these data. In addition, the subtest Planning was added to the visual-spatial processing broad factor $\mathrm{G} v$ for the sake of theoretical alignment, although it also loaded onto fluid reasoning (Gf) for a similar model fit. Cross-loading Planning onto both factors simultaneously decreased fit slightly, however. Underscoring the similarities between fluid reasoning and visual-spatial processing as measured by the WAIS-III/IV and WJ III, Matrix Reasoning also loaded readily on Fluid Reasoning $(\mathrm{G} f)$ as well as on VisualSpatial Processing $(\mathrm{G} v)$; however, model fit improved slightly when it was placed solely on Gv. This finding may be partially due to battery-specific effects from the WoodcockJohnson (WJ) subtests: The fluid reasoning (Gf) factor ended up consisting exclusively of WJ III tests with the small but significant cross-loading of the comprehensionknowledge (Gc) subtest Verbal Comprehension. This loading was considered theoretically consistent considering one of the tasks of Verbal Comprehension is analogies, a task that requires fluid reasoning as well as verbal knowledge.

A preliminary examination of the Planning subtest scores revealed several 
unusually high scores (i.e., >200) and the greatest skew (2.47) of all the subtests. To minimize the potential influence of this skew and the unusually high scores, Planning scores were transformed using a fourth-root transformation and calibrated to a mean of 100 and standard deviation of 15. As a result of this procedure, Planning's correlations improved with all subtests except Pair Cancellation. Another subtest, Visual-Auditory Learning, did not load well onto its typical learning and retrieval factor Glr. Without this subtest's anchor as a learning and retrieval test, Glr split into "Gr", or a "retrieval" factor, and "Gl", or a "learning" factor. The remaining subtests that typically load on Glr, Retrieval Fluency and Rapid Picture Naming, affiliated strongly with the processing speed factor Gs as speed-of-recall tasks. Gs thus effectively subsumed $\mathrm{G} r$ as a narrow factor. Finally, because they are essentially identical tests, model fit improved when Digit Span-Backward and Numbers Reversed were allowed a method covariance.

Both models illustrated results that also have been recently found and are supported by $\mathrm{CHC}$ theory: Fluid reasoning $(\mathrm{G} f)$ subtests typically had the strongest loadings on $g$ (Reynolds, Keith, Flanagan, \& Alfonso, 2013). In fact, subtest loadings on the $\mathrm{G} f$ broad factor were rendered nonsignificant in the bifactor model because of their high loadings on $g$. Also as expected, visual-spatial processing $(\mathrm{Gv})$ subtests also loaded strongly on $g$. Further, consistent with Reynolds and colleagues' findings, comprehension-knowledge (Gc) subtests did not load as strongly on $g$ as $\mathrm{G} f$ subtests did, but they loaded more highly on $g$ than the lower $g$-loaded subtests of processing speed $(\mathrm{Gs})$, working-memory capacity $(\mathrm{Gsm})$, and retrieval $(\mathrm{Gr} r)$. Similar to results obtained by Golay and colleagues (2012), the working-memory capacity (Gsm) and processing speed 
(Gs) factors showed strong independent loadings by their respective subtests.

In sum, the fits of the $g$ hierarchical and bifactor models suggest broad support for CHC theory with minor caveats. Since many psychologists use CHC theory to interpret test results, it is helpful to recognize that subtests are not necessarily measuring only one construct. Although these findings broadly validate $\mathrm{CHC}$ theory, provision must be made to recognize that difficulty with a particular subtest does not necessarily implicate only the broad factor to which that test is typically ascribed. However, the results of this study add to the convergent evidence that supports the validity of CHC theory and strengthens CHC theory-based interpretations.

\section{Model 2 - Hierarchical and Bifactor $\boldsymbol{g}_{f} \boldsymbol{g}_{c}$ Models}

The initial calibration $g_{f} g_{c}$ model began where the final calibration $g$ model ended, with the best-fitting broad/ narrow factor configuration from Model 1 combined with the broad factors reading and writing knowledge ( $\mathrm{Gr} w$ ) and quantitative knowledge $(\mathrm{G} q)$ in a model in which two general factors covary and account for separate groups of broad factors. Although the Root Mean Square Error of Approximation fit statistics indicated adequate model fit (RMSEA $=.046$ for the bifactor model), the Comparative Fit Indexes (CFI $=.930$ for the bifactor model) never approximated the CFI indexes of the $g$ models (CFI $=.960$ for the bifactor $g$ model). The $g f-g c$ model neither fit as well from the initial analysis as the $g$ model $\operatorname{did}(\mathrm{CFI}=.867$, SRMR $=.070$, $\mathrm{RMSEA}=.059)$, nor fit as well after adding the cross-loadings suggested by the exploratory analyses. Table 56 illustrates the fit statistics for the various final $g_{f} g_{c}$ models tested. The $g_{f} g_{c}$ correlation was quite high (.81 in the combined model) but did not reach unity, 
suggesting that these two constructs are indeed different.

Table 56

Hypotheses Testing $-g_{f} g_{c}$ Hierarchical and Bifactor Models

\begin{tabular}{|c|c|c|c|c|c|c|c|c|c|}
\hline Model/Sample & $n$ & $\chi^{2}$ & $d f$ & $\Delta \chi^{2 \mathrm{a}}$ & $\Delta d f^{\mathrm{a}}$ & $p$ & CFI & RMSEA & SRMR \\
\hline $\begin{array}{l}\text { Hierarchical } g_{f} g_{c} \\
\text { Calibration }\end{array}$ & 421 & 1327.5 & 663 & & & & .911 & .049 & .059 \\
\hline $\begin{array}{l}\text { Bifactor } g_{f} g_{c} \\
\quad \text { Calibration }\end{array}$ & 421 & 1166.0 & 624 & 161.55 & 39 & $<.001$ & .928 & .045 & .056 \\
\hline $\begin{array}{l}\text { Hierarchical } g_{f} g_{c} \\
\text { Validation }\end{array}$ & 444 & 1395.8 & 663 & & & & .916 & .050 & .060 \\
\hline $\begin{array}{l}\text { Bifactor } g_{f} g_{c} \\
\text { Validation }\end{array}$ & 444 & 1237.5 & 624 & 158.37 & 39 & $<.001$ & .930 & .047 & .059 \\
\hline $\begin{array}{l}\text { Hierarchical } g_{f} g_{c} \\
\text { Full Combined }\end{array}$ & 865 & 2043.4 & 663 & & & & .915 & .049 & .055 \\
\hline $\begin{array}{l}\text { Bifactor } g_{f} g_{c} \\
\quad \text { Full Combined }\end{array}$ & 865 & 1759.6 & 624 & 280.5 & 39 & $<.001$ & .930 & .046 & .055 \\
\hline
\end{tabular}

Part of the $g_{f} g_{c}$ model's difficulty may have been that the achievement tests were designed as outcome measures of the effects of cognitive functioning, as measured by the cognitive tests, rather than as direct measures of cognitive functioning. The exploratory analyses indicated that several of the achievement tests cross-loaded on their respective cognitive broad factors and vice versa. For example, the fluid reasoning (Gf) subtest Analysis-Synthesis cross-loaded on the quantitative knowledge factor, $\mathrm{G} q$, and Passage Comprehension cross-loaded on the comprehension-knowledge factor, Gc. Similarly, the comprehension-knowledge (Gc) subtests Verbal Comprehension and Vocabulary found small but significant cross-loadings on the reading and writing knowledge factor, Grw. Both the reading and writing fluency measures cross-loaded on the retrieval $(\mathrm{Gr} r)$ factor with Retrieval Fluency and Rapid Picture Naming. Fitting these data well to this model 
proved to be a difficult task. Figures 12 and 13 illustrate the hierarchical and bifactor $g_{f}$ $g_{c}$ models with factor loadings.

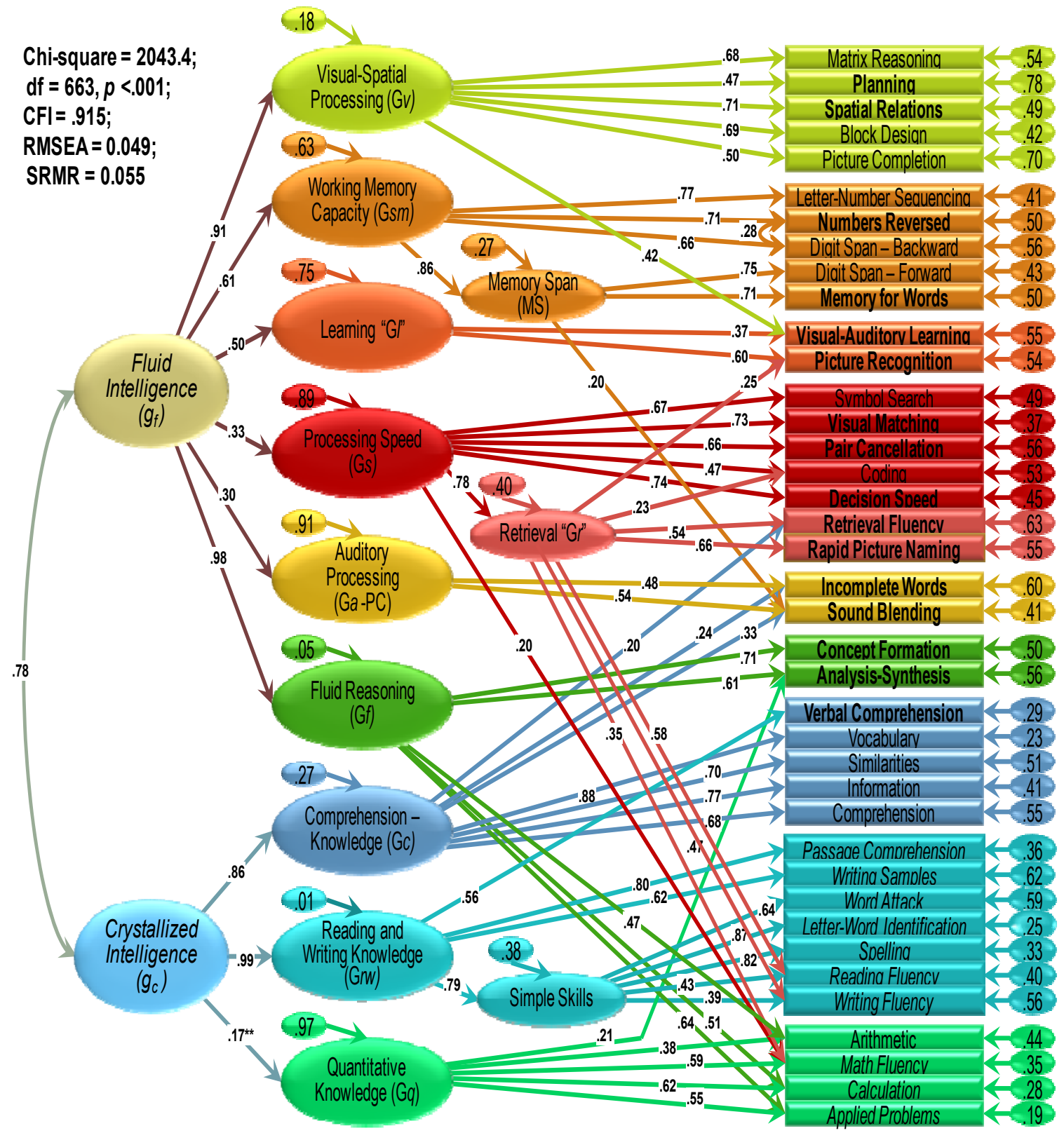

Figure 12. Final hierarchical $g_{f} g_{c}$ model of WAIS-III/IV and WJ III COG with CHC broad and narrow factors. WJ III cognitive subtests are noted in bold. WJ III achievement subtests are noted in italics. Completely standardized maximum likelihood parameter estimates. All estimates significant $p<.001$ except $* * p<.01$. 


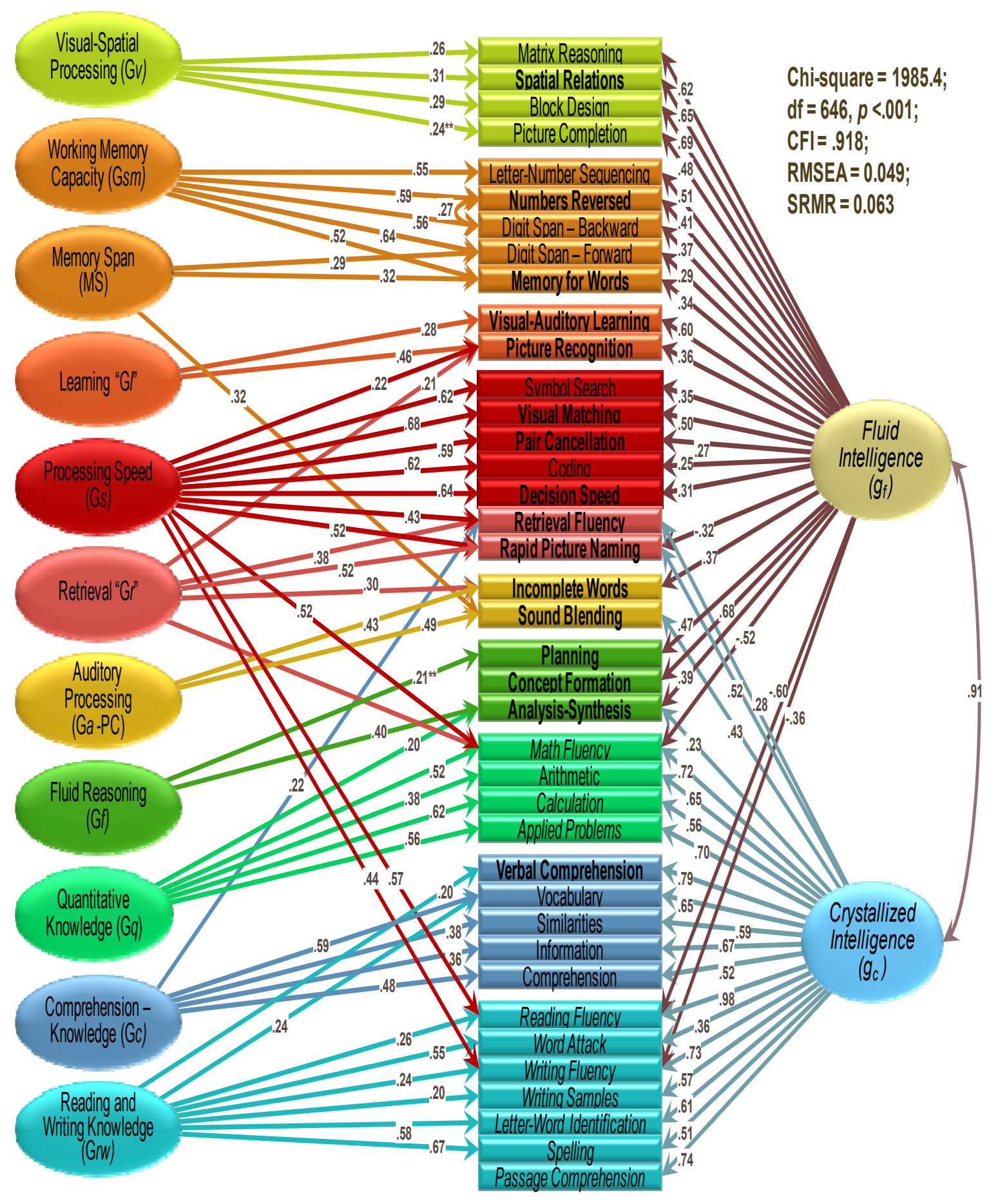

Figure 13. Final bifactor $g_{f} g_{c}$ model of WAIS-III/IV and WJ III COG with CHC broad factors. WJ III subtests are noted in bold. WJ III achievement subtests are noted in italics. Completely standardized maximum likelihood parameter estimates. All estimates significant $p<.001$ except $* * p<.01$. 


\section{Hypothesis 2 - Cognitive-Achievement Relations}

Because the $g_{f} g_{c}$ models provided poorer fits for the data than did the $g$ models, the decision was made to consider only the $g$ model for analyses concerning cognitiveachievement relations. After the revised $g$ model was confirmed by the validation and full combined datasets, the revised $g$ model was used as the primary measurement model for testing cognitive-achievement relations through structural equation modeling. To determine the best structural model for the achievement variables, parallel and exploratory analyses were performed on the calibration dataset achievement variables. Results indicated that three to four factors would provide a good fit for the achievement data, roughly divided into decoding or simple reading and writing skills (DEC), quantitative knowledge $(\mathrm{G} q)$, reading/writing $(\mathrm{G} r w)$, and fluency factors. Because these results did not provide a clean interpretation of the data, however, factor cross-loadings were allowed to provide a better fit for subtests that loaded on more than one factor (e.g., reading, writing, and mathematical fluency subtests). Items also cross-loaded on the bifactor model to accommodate the "flatness" of the model and subsume narrow factors. As with the $g$ and $g_{f} g_{c}$ cognitive models, the bifactor model provided the best fit for the data. Also, as with the previous models, once the optimal fit was determined for the calibration model, the model fit was validated successfully with both of the other datasets. The fit statistics for the final tested cognitive-achievement hierarchical and bifactor models for all three datasets are listed in Table 57. 
Table 57

Hypotheses Testing - $g$ Hierarchical and Bifactor Models as Achievement Predictors

\begin{tabular}{|c|c|c|c|c|c|c|c|c|c|}
\hline Model/Sample & $n$ & $\chi^{2}$ & $d f$ & $\Delta \chi^{2 \mathrm{a}}$ & $\Delta d f^{\mathrm{a}}$ & $p$ & CFI & RMSEA & SRMR \\
\hline $\begin{array}{l}\text { Hierarchical } g \\
\text { Calibration }\end{array}$ & 417 & 1619.7 & 671 & & & & .872 & .058 & .078 \\
\hline $\begin{array}{l}\text { Bifactor } g \\
\text { Calibration }\end{array}$ & 417 & 1331.9 & 646 & 287.86 & 25 & $<.001$ & .907 & .050 & .075 \\
\hline $\begin{array}{l}\text { Hierarchical } g \\
\text { Validation }\end{array}$ & 448 & 1594.6 & 671 & & & & .896 & .055 & .080 \\
\hline $\begin{array}{l}\text { Bifactor } g \\
\text { Validation }\end{array}$ & 448 & 1287.6 & 646 & 306.97 & 25 & $<.001$ & .928 & .047 & .061 \\
\hline $\begin{array}{l}\text { Hierarchical } g \\
\text { Full Combined }\end{array}$ & 865 & 2547.0 & 671 & & & & .885 & .057 & .075 \\
\hline $\begin{array}{l}\text { Bifactor } g \\
\text { Full Combined }\end{array}$ & 865 & 1985.4 & 646 & 561.63 & 27 & $<.001$ & .918 & .049 & .063 \\
\hline
\end{tabular}

As shown in Table 57, the overall model fit is poorer for the $g$ - achievement relations than it was for the $g$ model alone. The goal of the $g$-achievement model was to predict achievement from cognitive test scores; therefore, it was important to keep the achievement variables separate from the cognitive scores. When examining the $g-$ achievement model fit, the modification indices indicated that many of the cognitive variables had potential cross-loadings on the achievement factors and vice versa. For example, if allowed, the WJ III Verbal Comprehension subtest would have loaded as strongly on the complex reading and writing skills (CS) factor as on the comprehensionknowledge $(\mathrm{Gc})$ factor. Also, with the $g$-achievement bifactor model, the loadings on the fluid reasoning $(\mathrm{G} f)$ factor were not significantly different from zero. The $\mathrm{G} f$ factor was, therefore, subsequently excluded from the model. Figures 14 and 15 depict the structural models of the relevant regressions and parameter estimates involved in the prediction of 
the achievement variables for the hierarchical and bifactor models of cognitiveachievement relations, respectively.

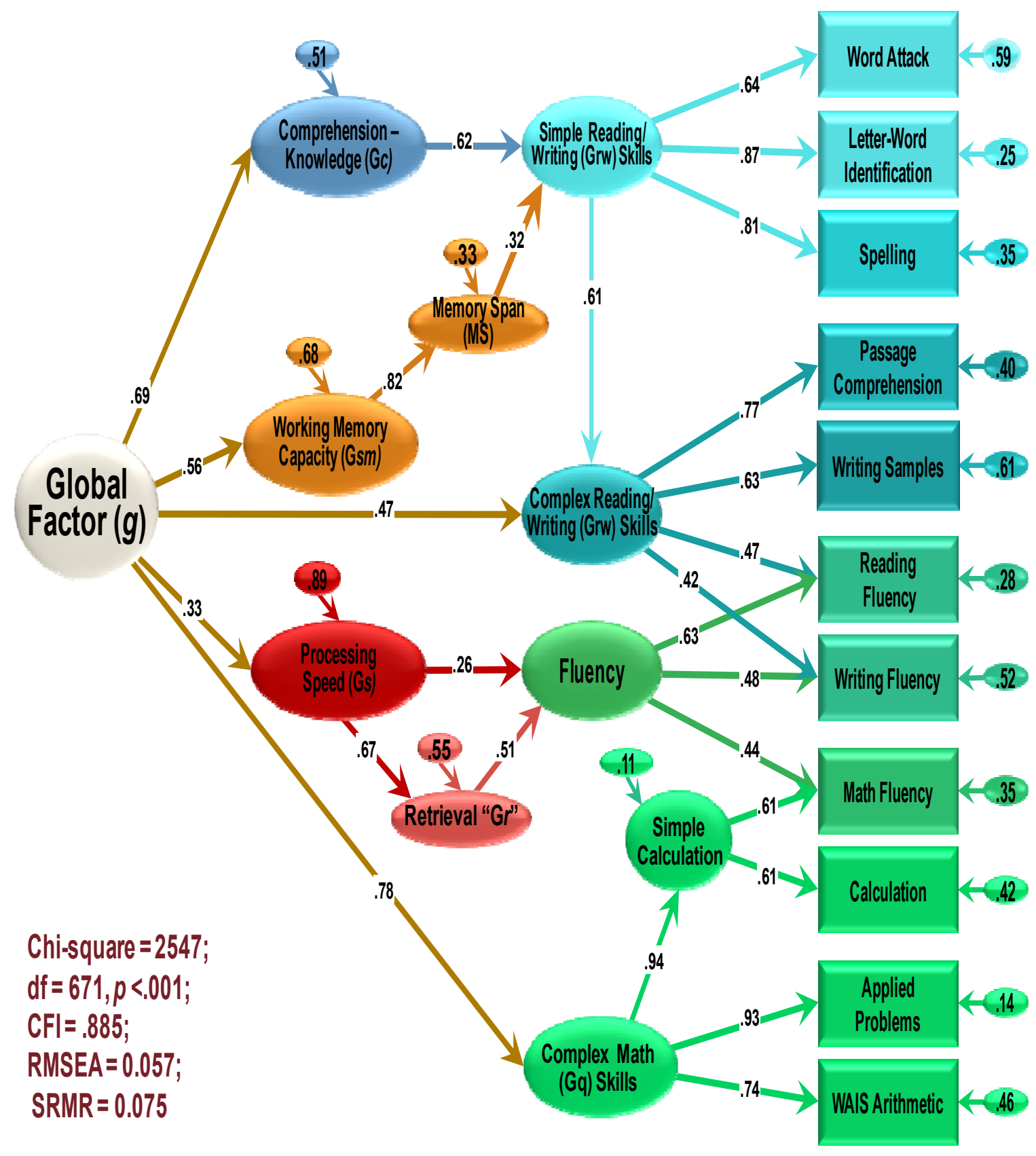

Figure 14. Final hierarchical cognitive-achievement structural model of WAIS-III/IV, WJ III COG, and WJ III ACH subtests with significant $\mathrm{CHC}$ broad and narrow factors. Completely standardized maximum likelihood parameter estimates. All parameter estimates and regressions significant $p=<.001$. 


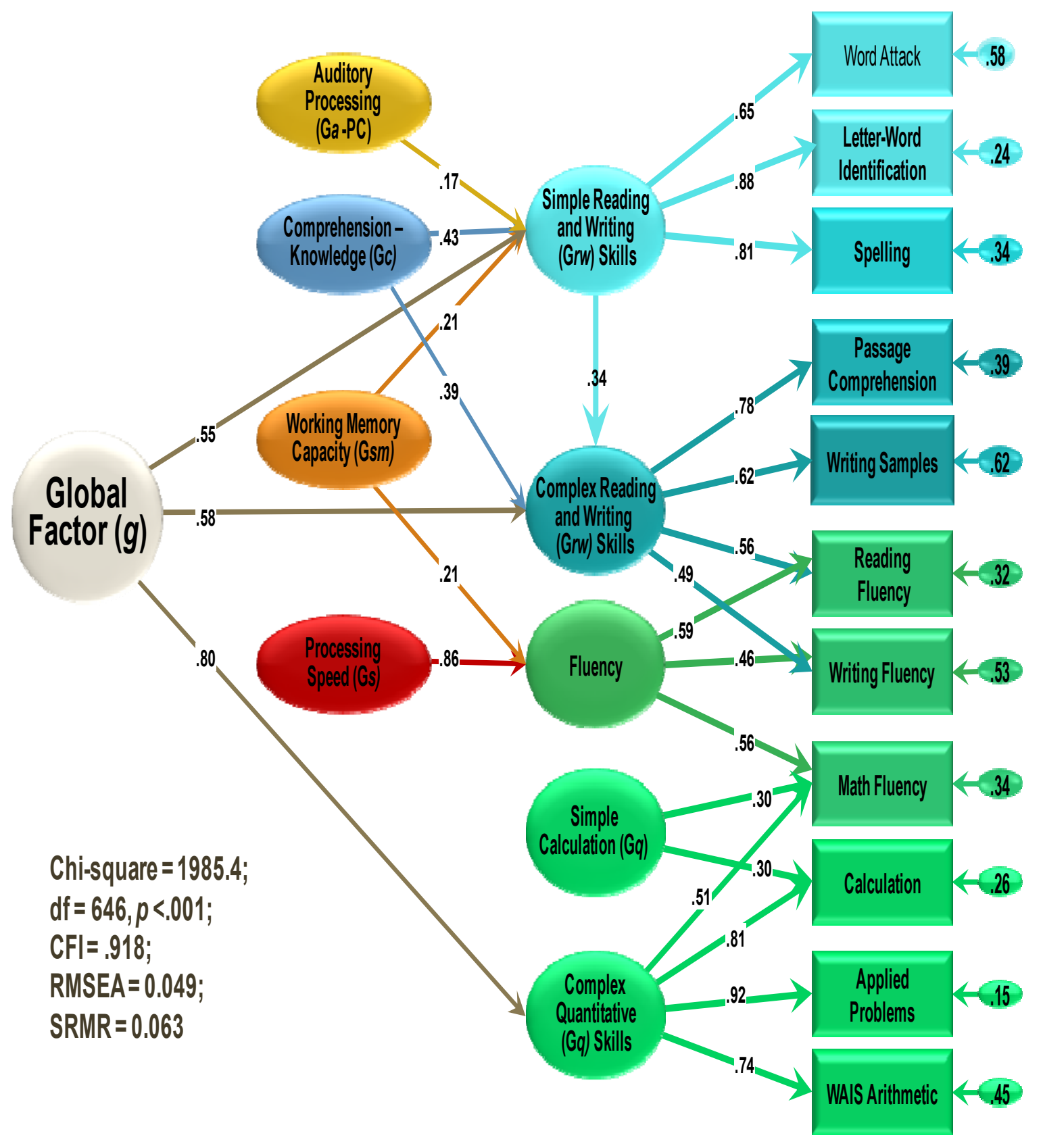

Figure 15. Final bifactor cognitive-achievement structural model of WAIS-III/IV, WJ III COG, and WJ III ACH subtests with significant CHC factors. Completely standardized maximum likelihood parameter estimates. All parameter estimates and regressions significant $p=<.001$.

For the final bifactor cognitive-achievement model, regressions for the achievement test scores were obtained from $g$ and the broad and narrow factors. The 
simple reading and writing (Grw) skills factor was highly significantly predicted by the overall factor $g(\beta=.55, p<.001)$, then by the comprehension-knowledge factor $\mathrm{Gc}(\beta=$ $.43, p<.001)$, by the working-memory capacity factor $\mathrm{G} s m(\beta=.29, p<.001)$, and by the auditory processing factor $\mathrm{G} a(\beta=.17, p=.001)$. The achievement fluency factor, consisting of Mathematics, Reading, and Writing Fluency, was significantly predicted by only two cognitive factors: most strongly by the processing speed factor $\mathrm{G} s(\beta=.86, p<$ $.001)$ and then by the working memory capacity factor $\mathrm{Gsm}(\beta=.21, p<.001)$. It is not surprising that the processing speed (Gs) and working-memory capacity (Gsm) factors predicting Fluency measure the important aspects of fluency of speed and memory. Predictably, Fluency appears not to be influenced directly by $g$. Further, the reading and writing knowledge (Grw) complex skills factor was significantly predicted by the general factor, $g,(\beta=.58, p<.001)$, the comprehension-knowledge factor $\mathrm{Gc}(\beta=.39, p<.001)$, and the simple reading and writing knowledge (Grw) skills factor DEC $(\beta=.34, p<$ .001). Mathematics Computation was significantly predicted only by the general factor, $g(\beta=.80, p<.001)$. All of these significant predictors make solid theoretical sense when recognizing the robust comprehension component in reading and writing skills and the strong fluid reasoning component in mathematics skills.

\section{Hypothesis 3 - Consistent Incremental Validity of Additional Test Scores Regardless of Subtest Score Variability}

My original hypothesis regarding the incremental validity of additional test scores regardless of subtest score variability assumed that the narrow factors I proposed testing would load on their empirically supported broad factors. Testing this hypothesis became 
problematic for this reason. The subtest Matrix Reasoning loaded on the visual-spatial processing broad factor $\mathrm{G} v$ and not on the fluid reasoning factor $\mathrm{G} f$ as originally hypothesized, thereby making it a potential confound in any analysis that included it as a narrow factor score for $\mathrm{G} f$. Thus, analyzing the $\mathrm{G} f$ factor for variability became problematic. The decision to forego analysis on $\mathrm{G} f$ meant that only one broad factor could be analyzed for subtest variability. Having a sample of two analyzed broad factors might spur reasonable discussion regarding subtest variability in cognitive testing, but considering a sample of only one would not allow any reasonable conclusions to be made regarding cognitive testing as a whole. Therefore, the decision was made to forego analysis of Hypothesis 3 altogether.

\section{Hypothesis 4 - The Benefit of Service Utilization on GPA}

Hypothesis 4 was designed to detect the extent to which a student's grade point average (GPA) changed with respect to the student's utilization of the disabilities center's resources. The hypothesis included the possible influence on GPA slope by students' cognitive abilities, the influence of initial psychological difficulty, and the extent to which the student accessed services at the disability resources center. At the time of proposal, the dataset contained only the GPA obtained the semester before testing and the GPAs for three post-evaluation semesters. Subsequent data gathering obtained the GPAs for every semester students attended the university making it possible to conduct a latent growth curve analysis of four semesters pre-evaluation and three semesters postevaluation. The latent growth curve analysis was conducted using complete observations of the cognitive and achievement variables, pairwise data for the GPA covariance matrix 
(since there were many more missing variables at the tails of the GPA data), and full information maximum likelihood estimation to accommodate missing data.

The latent variables employed for the growth curve analysis were modified for three principal reasons from the variables initially proposed: The cognitive and achievement variables were modified to reflect the cognitive and achievement models determined in the analyses of the first two hypotheses, the ADHD variables were altered to prevent difficulties with sample size, and the "Mental Health" and "Service Utilization" variables were modified due to difficulties with model fit. Instead of the WAIS Full-Scale IQ (FSIQ) and WJ III General Intellectual Ability (GIA) comprising the latent variable encompassing cognitive ability, the final bifactor $g$ and final bifactor $g$ achievement models allowed more robust models to estimate cognitive and achievement abilities. Rather than create the initially proposed "WJ III Reading Composite," "Writing Composite," and "Math Composite," the achievement model created by the $g-$ achievement analyses was employed for the "Achievement" latent variable and included "Simple reading and writing (Grw) skills," "Complex Grw Skills," "Simple Calculation," and "Mathematics Computation." The ADHD status variable was modified by using only the self-reported Conners' Adult ADHD Rating Scale (CAARS) subtests and not the CAARS other-rated scale because the substantially fewer number of completed CAARS other-rated scales would have unnecessarily reduced the sample size of complete observations.

To include students who were administered a structured clinical interview (SCID) in lieu of the Beck's Depression Inventory (BDI-II) and Symptom Checklist-90-Revised 
(SCL-90-R), mean scores of all students who received all three instruments were assigned to students who received the SCID alone (BDI-II $n=84, M=19.80, S D=$ 10.79; $\mathrm{SCL}-90-\mathrm{R} n=76, M=65.62, S D=10.48)$. This procedure was considered acceptable because there were no other discernible differences, other than time of evaluation, between students who received all three instruments and students who received only a SCID. The disability resource center's normal procedure during its early years of assessment was to administer all three instruments including a SCID when recommended by scores on the BDI, SCL-90-R, and as indicated in the initial academic screening interview (Collins, 2013, personal communication). This procedure was generally curtailed for brevity's sake in later years and students were administered a SCID based on results from the academic screening interview alone.

After including all model parameters, the final fit for the latent growth curve model was not as robust as hoped $(\mathrm{RMSEA}=.060, \mathrm{CFI}=.802)$. In fact, adding all parameters to the model at once produced a model that included negative variances and would not converge. Nonsignificant parameters were removed and "offending" latent variables were separated and re-added one indicator at a time. The "Mental Health" latent variable did not stand up to analysis: Attempts to shape the three variables "Beck Depression Inventory" (BDI-II), CAARS “Problems with Self-Concept Scale," and the Symptom Checklist 90-Revised (SCL-90-R) Global Severity Index (GSI) into a single mental health variable resulted in a model that generated negative variances. The single indicators were instead employed as "stand-ins" for the mental health variable, and models were tested that used each of the mental health variables alone or in combination. 
Similarly, the "Service Utilization" variable also did not work in the final model, perhaps due to difficulties with the "Number of Coaching/Counseling Sessions" indicator. Analysis of this variable detected that only 167 students of the 1,292 participants in the sample $(12.9 \%)$ had attended any coaching or counseling sessions. As a result, the distribution of this variable was skewed and extremely leptokurtic $(n=1292, M=1.35$, median $=0, S D=5.52$, Skew $=7.05$, Kurtosis $=65.61$, with the maximum number of sessions attended $=73$. Even when this variable was square-root transformed to reflect a more normal distribution, it proved to be a problematic indicator of service utilization because of the large percentage of students who did not use this service. As a result of the difficulties trying to use both observed variables as a single latent variable, an attempt was made to insert "Number of Coaching/Counseling Sessions" into the service model as a single service indicator. The other Service Utilization variable included whether the student received an accommodation letter which would allow the student to receive academic accommodations from the university. This variable proved difficult because no information was available to determine when the student received the letter or to what extent the student used the letter to receive accommodations. Nevertheless, an initial analysis revealed that just over half of the total sample of 1,292 students received an accommodation letter at some time during their academic careers $(n=703,54.4 \%)$, and thus this variable was also used alone as a service indicator.

The goal of the latent curve modeling was to predict the linear trend of students' GPAs over time with factors that might affect them. After receiving the additional GPA data, it was hypothesized that students' GPAs would decline leading up to testing 
(otherwise, why would they test?) and then improve post-testing as resources were accessed. Two slopes were incorporated into the model to reflect this hypothesis: Slope 1 included the four GPA variables including the GPA just before testing; Slope 2 included three post-test GPA variables in the latent curve analysis. A structural model of this analysis can be seen in Figure 16. Results of these analyses supported the main hypothesis in that GPAs showed a nonsignificant trend downward to testing and then a significant improvement after testing. However, the only significant regression in the final validation model, besides the regressions of the cognitive variables on the achievement variables as noted in Hypotheses 1 and 2, was a significant prediction of Graduate Student Status on the intercept, $\beta=.36, p<.001$. This arbitrary finding was expected since grading criteria are different for graduate students than for undergraduate students, with graduate GPAs significantly higher than undergraduate GPAs at the start. 


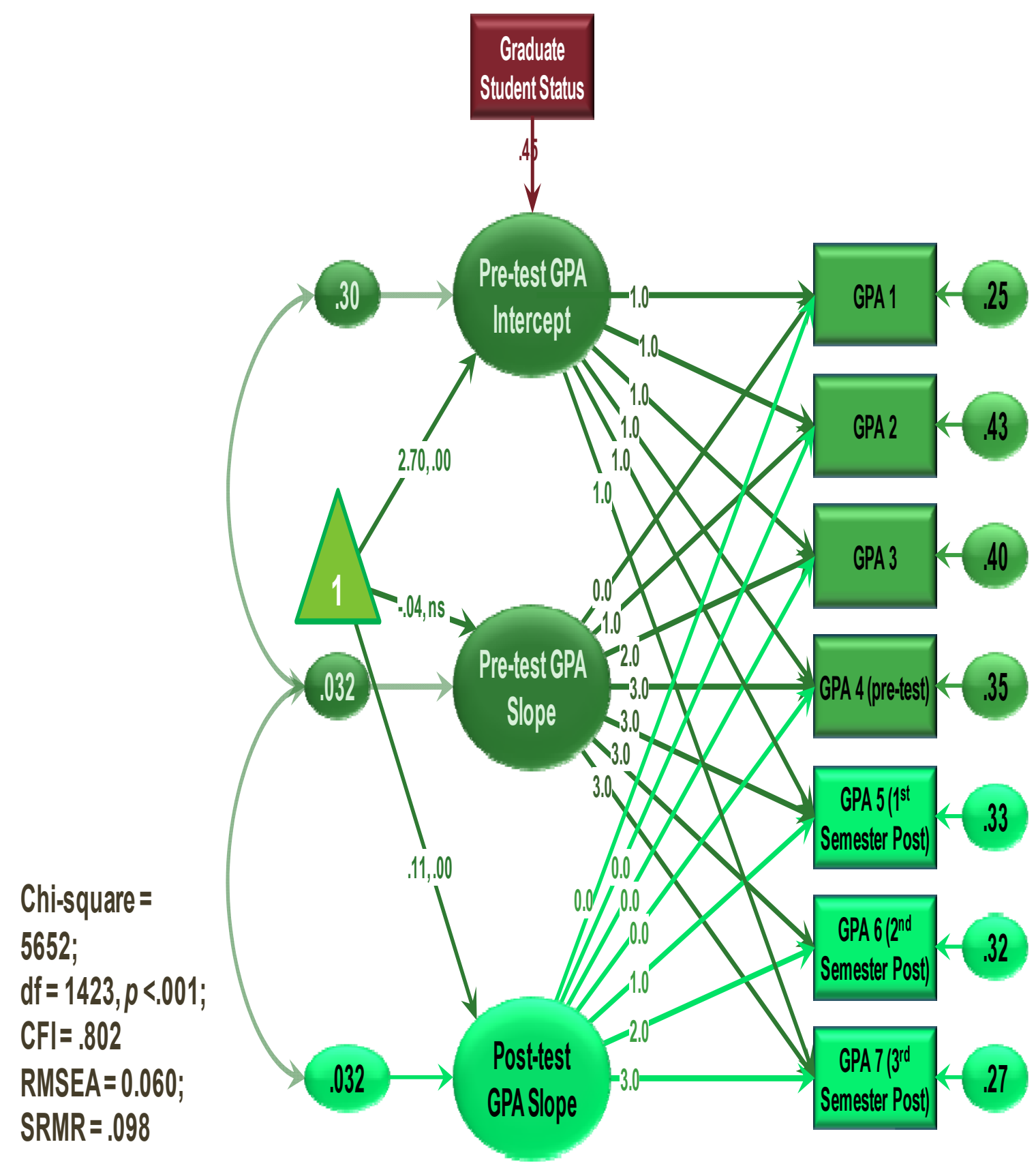

Figure 16. Service utilization model using latent growth curve analysis.

Two other indicators merit discussion, however. The SCL-90-R had a small but significant influence on GPA Slope 1 in the calibration dataset $(\beta=.11, p=.034)$, but this finding disappeared in the latent curve analyses of the validation and combined 
datasets. Additionally, the accommodation letter trended toward significance on the second slope $(\beta=-.15, p=.06)$ in the calibration dataset; however, this significance also disappeared in the validation and combined datasets.

To better understand these phenomena, the data with respect to the SCL-90-R and accommodation letter were analyzed with SPSS using two repeated-measures ANOVAs. Regarding the SCL-90-R, a within-subjects factor of the first four GPAs was compared to the between-subjects factor SCL-90-R. Mauchly's test indicated that the assumption of sphericity was not violated $\left(\chi^{2}(5)=10.23, p=.069\right)$. The effect of the SCL-90-R score on GPA slope over the first four semesters was significant, $F(126,663)=.274, p=.049$, $\eta_{\mathrm{p}}{ }^{2}=.191$. This finding suggests that psychological distress might be one contributing factor leading one to seek neuropsychological testing.

Regarding the accommodation letter, a within-subjects factor of 8 GPAs (with GPA 4 as the final GPA before testing) was tested with the between-subjects factor of accommodation letter (yes or no). Mauchly's test indicated that the assumption of sphericity had been violated $\left(\chi^{2}(27)=85.55, p<.001\right)$; therefore, degrees of freedom were corrected using Greenhouse-Geisser estimates of sphericity $(\varepsilon=0.72)$. A repeated measures test of within-subjects effects clearly showed a significant change in GPA, $\left.F(5.06,338.8)=5.39, p<.001, \eta_{\mathrm{p}}{ }^{2}=.074\right)$. Although there were significant differences in GPA, there was no influence for this change found in the acquisition of an accommodation letter, $F(5.06,338.8)=1.16, p=.33, \eta_{\mathrm{p}}{ }^{2}=.017$. Further, no significant differences were found when analyses were performed with a sample that excluded students who did not receive a diagnosis. An examination of the trajectories of GPA by 
people who received an accommodation letter compared with people who did not can be seen in Figure 17, where clear gains can be seen by both groups after testing. Other than the findings just explained, there was no evidence that other variables had significant influence over either of the GPA slopes yet, clearly, students' GPA trajectories reversed and improved after testing. Although one cannot assume a causal role of testing in the improvement of GPAs post-testing, the finding is remarkable nevertheless.

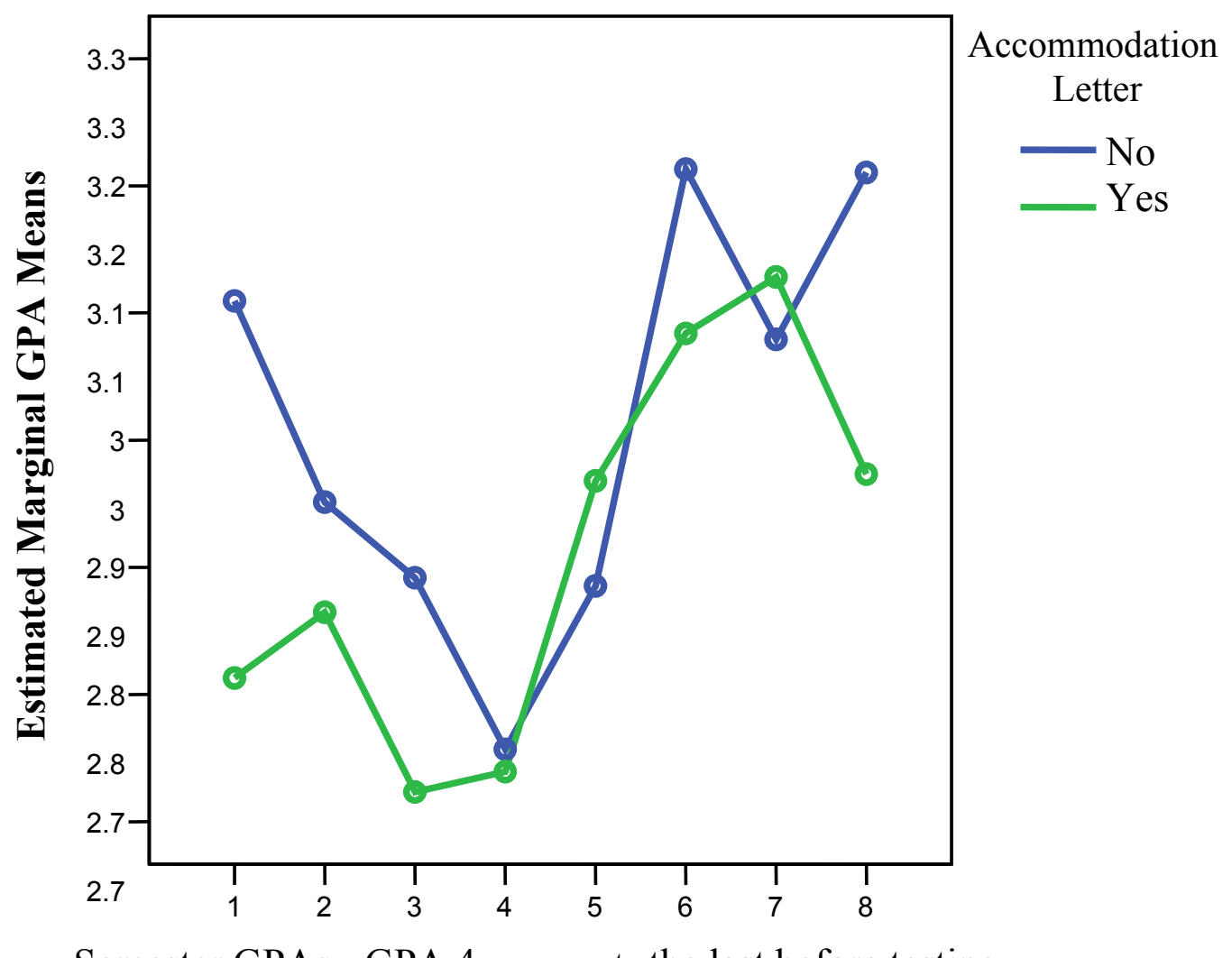

Semester GPAs - GPA 4 represents the last before testing.

Figure 17. GPA trajectories by whether a student received an accommodation letter. GPA trajectory begins four semesters prior to testing and continues four semesters after testing. 


\section{CHAPTER V \\ DISCUSSION}

The purpose of this study was to explore the relations among the cognitive, achievement, and mental health measures used to evaluate a sample of college students experiencing academic difficulty. Significant work has examined relations among cognitive and achievement measures with typically performing primary and secondary students (e.g., McGrew \& Wendling, 2010, Floyd et al., 2008), but less work has investigated the cognitive, achievement, and mental health relations in college students with suspected disabilities. Results of this study offer some practical and theoretical implications regarding these students but engender several additional questions.

This discussion is grouped by the research aim and proposed hypotheses and includes: 1) a review of the results with respect to the literature described in Chapter II and implications for theory and practice, 2) strengths and limitations of the study, and 3) general conclusions and directions for future study.

\section{Research Aim - Descriptive Analyses of the Demographic and Diagnostic Groups}

Before describing the individual demographic and diagnostic groups, it is important to acknowledge that no adjustment was made (e.g., Bonferroni correction) to account for the sheer number of comparisons conducted in this study. The goal of these comparisons was to provide a descriptive picture of the sample rather than a definitive 
statement of the relations among demographic and diagnostic variables and should be treated as such.

Some findings regarding the initial WAIS-III and WAIS-IV calibration datasets are also worth noting. First, with respect to the WAIS-III and WAIS-IV full-scale IQ (FSIQ) and WJ III General Intellectual ability (GIA) scores, there is a significant decrease in mean FSIQ from the WAIS-III to the WAIS-IV $(F(1,1180)=18.90, p<$ .001), perhaps reflecting the new norms calculated from the WAIS-III to the WAIS-IV and the manufacturer's desire to counter the Flynn effect (1987) of gradually increasing cognitive scores by making the WAIS-IV a more difficult test. This difference was not detected in the two databases' WJ III, $F(1,1161)=.861, p=.35$. Second, the WAISIII/IV FSIQ mean score was substantially higher than the WJ III GIA mean score in all three calibration datasets (WAIS-III, WAIS-IV, and Combined calibration datasets). The reason for this discrepancy remains unclear since both batteries use subtests from diverse cognitive areas to determine their respective full-scale scores: The WAIS FSIQ uses Similarities, Vocabulary, and Information (comprehension-knowledge); Block Design and Matrix Reasoning (visual-spatial processing); Arithmetic (fluid reasoning), Digit Span (working-memory capacity), and Symbol Search and Coding (processing speed); and the GIA uses Verbal Comprehension (comprehension-knowledge), Concept Formation (fluid reasoning), , Spatial Relations (visual-spatial processing), VisualAuditory Learning (learning), Sound Blending (auditory processing), Numbers Reversed (working-memory capacity), and Visual Matching (processing speed). 


\section{Gender}

No hypotheses were proffered regarding gender differences, but the differences found in this dataset are interesting in that they address the "urban myth" that women are better at verbal skills while men are better at quantitative skills, albeit with the caveat that both male and female referred college students may be atypical of the general population. The findings with this sample support half of this notion: The men performed better than the women at all of the mathematics-related tasks, including mathematical fluency, but they also performed better in working memory tasks and some of the comprehensionknowledge tests, including Verbal Comprehension and Information. The women did not "outdo" the men in any of the comprehension-knowledge tests, but they performed better than the men in all tasks related to fluency except mathematical fluency. The women also fared better than the men in the processing speed tasks and the auditory processing test of Sound Blending.

Women and men rated themselves equivalently on the CAARS scales for ADHD symptoms: Although women rated themselves higher than the men did on the CAARS inattention/memory problems, hyperactivity/ restlessness, and impulsivity/emotional lability, men rated themselves higher than the women on DSM-IV ADHD symptoms. There were also no discernible differences between men and women in actual subsequent ADHD diagnoses. Women received proportionally more diagnoses in general, however, and proportionally more diagnoses of depression and anxiety. The dataset consisted of more men $(N=706)$ than women $(N=586)$; perhaps women feel a greater level of psychological distress before they enter the disabilities resource center to be evaluated. 


\section{Ethnicity}

Again, no hypotheses were offered regarding ethnic differences, but some significant differences emerged. The African American group rated themselves lowest on the CAARS ADHD scores and received the lowest proportion of ADHD-Combined diagnoses, but they received the highest proportion of Verbal Learning Disability diagnoses of all groups. This diagnosis is congruent with the finding that the African American group also received the lowest cognitive and achievement scores, consistent with research that indicates that students with a verbal learning disability have particular problems with academic work because much of it requires a facility with language (Manalo et al., 2010). Consistent with Mrazik and colleagues' (2010) findings that students with learning disabilities also have difficulties with working and auditory memory, the African American group scored the lowest on the working memory subtest, Letter Number Sequencing, and among the lowest, with the Hispanic group, on the other working memory subtests Digit Span, Memory for Words, and Numbers Reversed. African Americans also scored lowest on Sound Blending, consistent with Compton and colleagues' (2012) finding that phonological problems significantly predict the manifestation of verbal learning disabilities in children.

\section{Primary diagnosis}

Much was hypothesized regarding the profiles of students in the various diagnostic groups, particularly with regard to cognitive-achievement profiles and the predictions of cognitive and academic difficulty given a particular diagnosis. Perhaps the most striking finding in this inquiry was the failure to replicate previous findings that 
people with depression tend to exhibit lower cognitive functioning overall (Francomano et al., 2011). In the current sample, the group of students receiving a primary diagnosis of depression performed as well as or better than all other groups, including the "no diagnosis" group, on every cognitive and achievement subtest. Despite the Depression group's superior performance on these batteries, however, no significant differences in pre-testing GPAs were found between students with and without primary Depression diagnoses.

In previous studies, students with ADHD demonstrated lower achievement levels overall (DuPaul et al., 2009; Frazier et al., 2007), as well as lower working memory, visual memory, and processing speed (Finke et al., 2011; Gropper \& Tannock, 2009; Nigg et al., 2005). Results from this study failed to replicate the findings of earlier work. The ADHD groups in this high-achieving sample performed as well as or better than all other groups on all achievement tests. Regarding the working memory subtests, the ADHD-C group was outperformed on Digit Span only by the students diagnosed with depression, and they performed similarly to students who received no diagnosis. The ADHD groups performed similarly to other groups on all other working memory tasks. In addition, the ADHD group obtained similar scores to all other groups except the Depression group on the FSIQ, GIA, and tests of visual memory, including Picture Completion and Picture Recognition. Finally, the ADHD group performed similarly to their non-ADHD peers on all processing speed tests. Barkley (2010) noted that the effects of ADHD are less noticeable with age, and especially with older students, not only because ADHD symptoms subside with adulthood, but also because students with the 
most severe symptoms tend to drop out of school. For the current sample, it appears that the students were able to use their cognitive strengths to compensate for their ADHD challenges and matriculate successfully into the university, only to struggle now that the work has become more rigorous than they were accustomed to in high school or undergraduate work.

As noted earlier with the African-American group, the students with Verbal Learning Disability (VLD) demonstrated the lowest reading and writing (Grw) achievement scores of all groups. Again, this finding is not surprising given the heavy emphasis on verbal and written language in these areas of academic achievement. The VLD group fared relatively poorly on all comprehension-knowledge subtests for the same reason. The VLD group also obtained the lowest scores of all groups on the specific auditory processing subtest Sound Blending, consistent with Bone and colleagues (2002) finding that the narrow ability Ga-Phonemic Awareness (which Sound Blending measures) differentiates students with and without reading disabilities regardless of whether they have an overall IQ-achievement discrepancy. As expected, the VLD group performed similarly to their non-VLD peers in the "non-language" fluid reasoning and visual-spatial processing $(\mathrm{G} f / \mathrm{G} v)$ and processing speed $(\mathrm{G} s)$ subtests.

The Nonverbal Learning Disability (NVLD) group experienced the opposite difficulties from the VLD group. The NVLD group fared relatively well on the comprehension-knowledge/reading and writing knowledge ( $\mathrm{Gc} / \mathrm{Grw})$ subtests but had greater difficulty with the fluid reasoning/visual-spatial processing/quantitative knowledge $(\mathrm{G} f / \mathrm{G} v / \mathrm{G} q)$ and processing speed $(\mathrm{G} s)$ subtests. An important consideration, 
however, is that even though the VLD and NVLD groups shared the lowest FSIQ and GIA group as a result of their difficulties with the highly $g$-loaded $\mathrm{G} c$ and $\mathrm{G} f / \mathrm{G} v$ subtests, their mean FSIQ and GIA scores of approximately 108 and 99, respectively, still place them solidly in the average range for all adults. As these groups are only now being assessed for a learning disability, they, like their ADHD peers, have heretofore been able to overcome their academic challenges with their good overall cognitive abilities and are only now experiencing difficulty with the increased academic rigor.

The students who struggled with a foreign language were not studied relative to the other diagnoses because they were not necessarily diagnosed with any particular disorder. The Foreign Language Learning Difficulty (FLLD) students were compared instead to their peers who underwent evaluation but did not request a foreign language substitution. Results of past studies suggest that students who struggle to learn a foreign language experience deficits relative to their non-affected peers on tests measuring phonetic coding and general native language ability (e.g., Carroll, 1990; Ferrari \& Palladino, 2007; Sasaki, 2012), working memory (i.e., Gsm; Andersson, 2010), fluid reasoning (i.e., Carroll, 1990; Riesiewicz, 2008), spelling (Scott et al., 2009; Sparks et al., 2006), and vocabulary (Riesiewicz, 2008). Results of this study generally replicate those findings, with lower scores than non-FLLD peers on most comprehension-knowledge, working-memory capacity, and fluid reasoning/visual-spatial processing subtests. In addition, the FLLD group's scores were lower than those of their non-FLLD peers on the auditory processing subtest Sound Blending. The FLLD group's lower cognitive scores yielded a highly significant reduction in all achievement test scores relative to their non- 
FLLD peers. However, as with the other learning disabilities, it is important to keep these results in perspective by realizing that the students with FLLD still performed in the average range (FSIQ $M=108, S D=13.19$; GIA $M=96.63, S D=11.70$ ) with respect to normal adults.

The various demographic and diagnostic groups have at least one variable in common: They all performed in the average to high-average range in cognitive functioning. Future studies might examine this phenomenon to examine specific differences in the ways these students manifest and manage their difficulties in the midst of relatively strong overall cognitive functioning.

\section{Hypothesis 1 - Factor Analyses of the WAIS-III/IV and WJ III Cognitive and Achievement Subtests}

With a few remarkable exceptions, all subtests loaded on their theoretically hypothesized factors, supporting CHC theory in general and its overall validity for this sample. The exceptions included a split of the long-term storage and retrieval Glr factor into "Gr," or a retrieval factor, and "Gl," or a general learning factor. Visual-Auditory Learning - Delayed was not administered to this group, so it was not possible to assess the delayed recall capability of this sample. Once the $\mathrm{G} l r$ factor split, $\mathrm{G} r$, comprised chiefly of speeded recall tasks, could be conceptualized as a narrow factor subsumed by processing speed (Gs). Also for this sample, Matrix Reasoning did not have a significant loading on fluid reasoning $(\mathrm{G} f)$ as it does in most studies. If one examines the $\mathrm{G} f$ factor in this sample, one notices that it comprises only Woodcock-Johnson (WJ III) tests (Analysis-Synthesis, Concept Formation, and Verbal Comprehension). Perhaps there is a 
battery effect that precludes Matrix Reasoning from taking its usual place within $\mathrm{G} f$. Also, many students were administered the WAIS and WJ III on separate days, which may differentiate how the different subtests were negotiated. It might also be the case that, in general, this sample of students handles the Matrix Reasoning subtest task differently than does the normal population. Future studies might illuminate the reasons Matrix Reasoning "behaved" differently with this sample.

Another notable finding from the factor analyses was the discovery that the bifactor model provided a significantly better fit for the data than did the hierarchical $g$ model. The bifactor model fit better because it recognizes that, within a broad factor, different indicators do not typically have the same ratio of $g$ variance to broad factor variance, and it allows them to vary. The proportionality constraints of the hierarchical model require that the proportions of $g$ variance within a broad factor remain the same for all of the particular broad factor indicators. The tradeoff of a bifactor model is that it reduces the degrees of freedom found in the hierarchical model. The hierarchical model also honors the necessary combination of $g$ and the broad factors in the expression of a particular ability. It might be conceptually difficult to think of a "residualized" ability as the bifactor model appears to exhibit. Practically, however, the bifactor model displays the independent influences of $g$ and the broad factors on the subtests.

Following Golay and colleagues' (2012) lead by allowing subtests additional factor loadings increased model fit and may have provided a more accurate look at the true complexity of the cognitive abilities tapped by the individual subtests. For example, Sound Blending, a subtest that primarily measures auditory processing, cross-loaded onto 
comprehension-knowledge (Gc), and short-term memory (Gsm-MS). These loadings make theoretical sense if one thinks about the work entailed in assembling heard speech sounds to create recognized words. One has to know the words $(\mathrm{Gc})$, and one has to remember what one just heard to do something with the sounds (Gsm-MS). Then one has to translate, or process, the sounds just heard, remembered, and recognized. The loadings from the bifactor model reflect the relative importance of $g$ and each of the factors on this task: .47 for $g, .47$ for $\mathrm{G} a, .26$ for $\mathrm{G} c$, and .32 for Gsm-MS (all $p<.001$ except MS, which is $p<.01)$. The other auditory processing ( $\mathrm{G} a$ ) task, Incomplete Words, loaded onto $\mathrm{G} c$ as well but loaded onto the retrieval factor, $\mathrm{G} r$, instead of on Gs. This loading also makes sense when considering the task required by Incomplete Words - recall a known word after hearing only parts of it. In sum, the small cross-loadings are present and, cumulatively, they matter. Confirmatory factor analyses that did not include these crossloadings likely misestimated some of the achievement relations they examined.

Finally, contrary to the hypothesized model, no narrow factors emerged in the analysis except short-term memory (MS). The question arises regarding why more narrow factors failed to emerge. Perhaps there were too few specific indicators of each narrow factor to allow the factor to emerge. The specificity of the subtests on each narrow or broad factor did not appear to be as clear cut with this sample as has been found in previous work, with many subtests loading on several factors. It is useful for test producers to pay attention to narrow factor loadings to provide diversity within the broad factors. For this sample, however, no evidence of the utility of the narrow factors in assisting diagnoses was found. 
To support their argument for CHC theory's endorsement of a general factor and specific and independent broad abilities, Floyd and colleagues (2009) found in their sample's young adult age groups that the comprehension-knowledge factor $\mathrm{G} c$, the longterm retrieval factor $\mathrm{G} l r$, and the fluid reasoning factor $\mathrm{G} f$ loaded primarily on the general factor, showing higher $g$ loadings than specificity effects, while the visual-spatial factor $\mathrm{G} v$, auditory processing $\mathrm{G} a$, working memory capacity $\mathrm{G} s m$, and processing speed $\mathrm{G} s$ demonstrated greater specific effects and lower $g$ loadings. Their findings were only partially supported in this sample, but this study's results support their view that $g$ as well as broad and narrow factors are needed to model cognitive functioning accurately. Differences in this sample from the Floyd et al. study include the breakup of Glr into Gl and $\mathrm{G} r$, whereby one subtest, Visual-Auditory Learning (VAL) is heavily $g$-loaded (.62), and shows lighter specific effects (.29), and the other Gl subtest, Picture Recognition (PR), shows a lighter $g$-loading (.37) but heavier specific effects, in part because it has been allowed to load onto two additional factors in the bifactor model: $\mathrm{Gr}(.20)$ and Gs (.20; all $p<.001)$. Also for this sample, all the visual-spatial processing (Gv) subtests are highly $g$ loaded and demonstrate marginal specificity effects, failing to replicate Floyd and colleagues' (2009) findings. Congruent with Floyd and colleagues' work, workingmemory capacity (Gsm) and processing speed (Gs) demonstrate higher specific loadings and lower $g$ loadings, which is made especially evident in the bifactor model.

Some difficulties with this investigation need to be reported: Revisions to the WAIS-IV were specifically designed to add some fluid reasoning/visual-spatial processing $(\mathrm{G} f / \mathrm{G} v)$ "heft" to the WAIS-IV and align it more closely with CHC theory 
(Drozdick et al., 2012). These revisions included the addition of the fluid reasoning (Gf) subtest Figure Weights and the visual-spatial processing (Gv) subtest Visual Puzzles. Regrettably, combining the WAIS-III and WAIS-IV made analyzing the new WAIS-IV tests impossible. If these subtests could have been added, Matrix Reasoning might have loaded onto a broader fluid reasoning $(\mathrm{G} f)$ factor, Visual Puzzles would likely have loaded onto the visual-spatial processing $(\mathrm{G} v)$ factor, and the posited possible battery effects of the WJ III might have been illuminated.

There was also something unusual in the WAIS/WJ III combination in the working-memory capacity (Gsm) factor. Analyzing the separated Digit SpanForward/Digit Span Backward created a differentiation from Letter-Number Sequencing that may have precluded a strong working memory (MW) narrow factor to emerge. The short-term memory (MS) subtests Memory for Words and Digit Span - Forward created a fairly straightforward MS narrow factor; however, Numbers Reversed and Digit Span Backward displayed a method covariance (being virtually the same test) and tended to exclude Letter-Number Sequencing, so the MW factor did not emerge as expected.

Regarding $g_{f} g_{c}$ theory and the model that represents it, Kaufman and colleagues (2012) found that Cognitive $g$ (represented by $g_{f}$ ) and Achievement $g$ (represented by $g_{c}$ ) are separate but highly correlated constructs. This study replicates their finding in that the covariance between $g_{f}$ and $g_{c}$ was also high but not unitary (.78 in the hierarchical model; .91 in the bifactor model.) However, $g_{f}$ and $g_{c}$ turned out not to be the truly discrete measures of cognitive functioning they were originally hypothesized to be: $g_{f}$ and $g_{c}$ subtests had many cross-loadings, making it difficult to differentiate some aspects of $g_{c}$ 
from $g_{f}$, particularly in fields of academic endeavor requiring highly $g$-loaded tasks, such as mathematics, where fluid reasoning $(\mathrm{G} f)$ and quantitative knowledge $(\mathrm{G} q)$ are closely related. As with the $g$ model, subtests cross-loaded on multiple factors which, while enhancing fit, complicated both the hierarchical and bifactor models. Nevertheless, allowing the additional loadings provided another opportunity to consider the complexity of cognition for which truly adequate models have yet to emerge. The fit for the $g_{f} g_{c}$ model, while adequate, did not compare favorably with the fit for the unitary $g$ model. Therefore, the $g$ model was used as a basis for all subsequent analyses.

\section{Hypothesis 2 - Cognitive-Achievement Relations}

After fitting the achievement variables to the $g$ model as regressions, overall model fit declined for both hierarchical and bifactor models. Although the bifactor model provided the best fit for the data as it did in the other models, fit difficulties with both models included the tendency of several comprehension-knowledge $(\mathrm{Gc})$ and fluid reasoning $(\mathrm{G} f)$ variables to load on the reading and writing knowledge $(\mathrm{G} r w)$ and quantitative knowledge $(\mathrm{G} q)$ factors. Maintaining fidelity to the model required keeping the achievement variables separate from the cognitive variables, however. Also, in the bifactor model, $g$ accounted for such a substantial amount of the variance from the tests that typically load on fluid reasoning $(\mathrm{G} f)$ that $\mathrm{G} f$ disappeared from significance in the model.

For the final model, consistent with predictions from McGrew and Wendling's (2010) large meta-analysis concerning the 14 to 19 age group and Floyd and colleagues' (2008) examination of writing in the WJ III norming sample, comprehension-knowledge 
$(\mathrm{Gc})$ predicted both simple and complex skills in reading and writing. In addition, $g$ directly predicted both simple and complex skills, perhaps serving as a proxy for the tentative fluid reasoning $(\mathrm{G} f)$ prediction cited by McGrew and Wendling. Also consistent with McGrew and Wendling's findings, (although they did not specifically examine writing skills) are the significant predictions of auditory processing and working-memory capacity to simple reading and writing skills. The current analysis found that processing speed and working-memory capacity also predict fluency as defined by the Math, Reading, and Writing Fluency subtests of the WJ III ACH. This finding is consistent with Benson's (2008) analysis of the WJ III standardization sample which found processing speed's increasing effects with age on fluency, and with Floyd and colleagues' (2008) examination of writing in which they posited that processing speed facilitates complex writing tasks by allowing basic skills to become automated. Floyd and colleagues also found working-memory capacity to be a moderate predictor of both basic writing skills and written expression and posited that it did so by facilitating the simultaneous management of verbal information and writing strategy resources.

Regarding the simple and complex mathematics skills, only $g$ predicted complex mathematics skills in the current study, and no specific predictors were found for the simple mathematics skills. Although the finding of $g$ or fluid reasoning as either a direct or indirect predictor of mathematics skills is somewhat consistent with other studies (Osmon et al., 2006, Proctor, 2012), these findings failed to replicate the other studies' findings of other significant predictors, including auditory processing, processing speed, working-memory capacity, comprehension-knowledge, and long-term storage and 
retrieval. This failure may be an artifact of the measured factors. Although the current study's model was able to clarify the role of simple skills in the acquisition of complex reading skills, it was unable to clarify this role for the mathematics factor, perhaps because there were no analogous and distinct "simple skills" for mathematics other than Math Fluency. Although two of the mathematics subtests factored into a narrow "simple calculation" factor, this was a residual factor after the general Gq (quantitative ability) factor was accounted for. Because of model convergence problems, several hypotheses about the differential effects of specific cognitive abilities on simple calculation and complex quantitative reasoning could not be tested. It is likely that having more indicator variables in the quantitative knowledge domain would result in fewer model convergence problems.

\section{Hypothesis 3 - Incremental validity with Subtest Variability}

This hypothesis was not testable because the fluid reasoning/visual-spatial processing $(\mathrm{G} f / \mathrm{G} v)$ and working-memory capacity (Gsm) subtests failed to differentiate into narrow factors as expected.

\section{Hypothesis 4 - The Benefits of Service Utilization}

Using qualitative methodology, Allsop and colleagues (2005) suggested that students differentially benefit from their access to services depending on cognitive abilities, initial academic achievement, psychological functioning, and service dosage. The results of this study failed to support these hypotheses. Results of latent growth curve modeling of students' GPA slopes suggest that GPA improves after testing, but the degree of improvement could not be predicted from any cognitive variable. There was no 
evidence that achievement variables predicted the intercept or either of the GPA slopes. Similarly, no evidence was found that mental health variables predicted any of the GPA variables. It appears that GPA improvement is not a simple function of cognitive or academic ability, nor is it a function of omnibus measures of mental health. The degree of improvement in GPA may be the result of the interaction of transient environmental influences and subtle effects of motivation that were not measured in this study. From the GPA slope changes, it is clear that students were tested during perceived crisis when their GPAs had dropped. It is possible that after testing students naturally regressed to their own mean regardless of testing. There was no way to test this hypothesis, however, since this study had no control group with which to compare results.

\section{Study Strengths and Limitations}

This study is remarkable for its relatively large dataset acquired over a long period of time. The size of the dataset allowed the legitimate exploration of complicated models that included many parameters. In addition, the administration to the same individuals of two relatively complete major cognitive batteries is fairly unusual and also allowed more complex models in the exploration of $\mathrm{CHC}$ theory and cognitiveachievement relations. Finally, access to longitudinal outcomes, expressed as the students' GPAs over several semesters pre- and post-testing, provided an extraordinary glimpse of students' trajectories before and after they were evaluated.

There were many limitations of this study that are equally noteworthy. Because the study was archival, the sample was one of convenience. Generalizability of the findings will be limited because the sample consisted primarily of bright young 
undergraduate and graduate students at a competitive university. None of the traditional experimental rigors could be employed in this study because of its archival nature. Not having control groups, either of students with suspected disabilities who did not come into the disabilities resource center to be tested, or of a matched sample of students without suspected disabilities, precluded being able to determine the extent to which students' GPA trajectories were unique to the students who were evaluated by the disabilities resource center. Not counterbalancing test administrations clouded the ability to ascertain the presence or extent of possible battery or order effects of testing. Finally, being unable to examine the WAIS-IV subtests Figure Weights and Visual Puzzles because of the small WAIS-IV sample size prevented a thorough exploration of the unusual loading of the WAIS Matrix Reasoning subtest on visual-spatial processing.

\section{General Conclusions and Directions for Future Study}

Results of this study provided cognitive, achievement, and mental health descriptions of the major demographic and diagnostic groups that are evaluated by a major Midwestern university's disabilities resource center for psychological disorders or learning disabilities. Several of the findings are consistent with past research regarding these groups, such as the interesting dichotomies between students with Verbal Learning Disabilities and students with Nonverbal Learning Disabilities, although other analyses failed to replicate the results of previous studies. Particularly notable in this latter group are the findings that the students diagnosed primarily with Depression obtained the highest cognitive and achievement scores of all groups and that the students diagnosed with ADHD scored as well as or better than all other groups except the Depression group 
on the cognitive and achievement variables. These findings may confirm the unusual nature and limited generalizability of this sample. These findings might also illuminate similar inquiries at other college disability resource centers, however, where comparable students are likely to be evaluated.

Further, this study's results are broadly consistent with and support $\mathrm{CHC}$ theory, but there are a number of findings that future $\mathrm{CHC}$ work should seek to address. In this study, the roles of the global factor $g$ and fluid reasoning $\mathrm{G} f$ were unclear. These abilities appear to be more intertwined than $\mathrm{CHC}$ theory would suggest and clarifying the distinct constructs of $g$ and $\mathrm{G} f$ would be a helpful endeavor for future work. Because there was only one learning subtest (Visual-Auditory Learning), understanding the unusual factoring that occurred with the processing speed, retrieval, and learning subtests is difficult. The learning broad factor $\mathrm{G} l$ consisted of two disparate tests (with the traditionally visual-spatial processing subtest Picture Recognition) and excluded loadings from the subtests that more traditionally load on long-term storage and retrieval $(\mathrm{Glr})$, the $\mathrm{G} r$ retrieval subtests. With the $\mathrm{G} r$ subtests providing a narrow factor for processing speed (Gs), the current study's findings suggest that Gr and Gs as constructs are not as distinct as $\mathrm{CHC}$ theory presently proposes. These factors could be clarified with additional learning tests in future cognitive batteries.

The cognitive-achievement relations found in the current study generally replicate the relations found in previous work. One potentially noteworthy area of future study would be to examine the likelihood that broad and narrow factors matter more when $g$ is high than when it is low. Low $g$ is likely the predominant factor affecting academic 
achievement when $g$ is low. However, as may be the case with this sample, the broad and narrow factors may play a more significant role when $g$ is high. This potential $g$ by broad/narrow factor interaction in predicting academic outcomes should be examined in future work.

The predictions regarding the cognitive, achievement, and mental health factors affecting academic performance were not confirmed. However, it is possible that there are significant latent variable interactions that were not explored in the service utilization model. I did not examine cognitive abilities and achievement as potential moderators of service utilization's influence on GPA slope. Future work exploring these latent variables might show that these interactions matter, although large effects predicting who will improve the most as a result of using the center's resources would likely have been found by this study.

Not identifying any significant predictors certainly warrants future work in this area but, in the meantime, may actually be viewed in a positive light. Results of this study indicate that students being evaluated by the center are likely to improve regardless of their initial cognitive or achievement scores, and regardless of the extent or nature of their initial psychological challenges. Whether improvement comes as a result of being evaluated cannot be concluded by this investigation, but the finding that improvement does occur after coming into the center should be welcome news to students and clinicians alike. 


\section{REFERENCES}

Abu-Hamour, B., Al Hmouz, H., Mattar, J., \& Muhaidat, M. (2012). The use of Woodcock-Johnson tests for identifying students with special needs - A comprehensive literature review. Procedia - Social and Behavioral Sciences, 47, 665-673.

Alfonso, V. C., Flanagan, D. P., \& Radwan, S. (2005). The impact of the Cattell-HornCarroll theory on test development and interpretation of cognitive and academic abilities. In D. P. Flanagan \& P. L. Harrison (Eds.), Contemporary intellectual assessment: Theories, tests, and issues ( $2^{\text {nd }}$ ed., pp. 185-202). New York: Guilford Press.

Allsop, D. H., Minskoff, E. H., \& Bolt, L. (2005). Individualized course-specific strategy instruction for college students with learning disabilities and ADHD: Lessons learned from a model demonstration project. Learning Disabilities Research \& Practice, 20, 103-118.

American Psychiatric Association. (2000). Diagnostic and statistical manual of mental disorders ( $4^{\text {th }}$ ed., text rev.). Washington, DC: Author.

Barkley, Russell A. (2010). ADHD in adults: What the science says. New York: Guilford Press. Barnard-Brak, L., Davis, T., Tate, A., \& Sulak, T. (2009). Attitudes as a predictor of college students requesting accommodations. Journal of Vocational Rehabilitation, 31, 189-198.

Barnard-Brak, L., \& Lan, W. Y. (2010). Analyzing disability accommodation statements. Journal on Excellence in College Teaching, 21, 29-47.

Barnard-Brak, L., Lechtenberger, D., \& Lan (2010). Accommodation strategies of college students with disabilities. The Qualitative Report, 15, 411-429.

Barnard-Brak, L., Sulak, T., Tate, A., \& Lechtenberger, D. (2010). Measuring college students' attitudes toward requesting accommodations: A national multiinstitutional study. Assessment for Effective Intervention, 35, 141-147.

Benson, N. (2008). Cattell-Horn-Carroll cognitive abilities and reading achievement. Journal of Psychoeducational Assessment, 26, 27-41. 
Benson, N., Hulac, D. M. \& Kranzler, J. H. (2010). Independent examination of the Wechsler Adult Intelligence Scale-Fourth Edition (WAIS-IV): What does the WAIS-IV measure? Psychological Assessment, 22, 121 - 130.

Bentler, P. M., \& Bonett, D. G. (1980). Significance tests and goodness-of-fit in the analysis of covariance structures. Psychological Bulletin, 88, 588-606.

Bickley, P. G., Keith, T. Z., \& Wolfe, L. M. (1995). The three-stratum theory of cognitive abilities: Test of the structure of intelligence across the life span. Intelligence, 22, 311-326.

Bone, R. B., Cirino, P., Morris, R. D., \& Morris, M. K. (2002). Reading and phonological awareness in reading-disabled adults. Developmental Neuropsychology, 21, 305320.

Bowden, S. C. (2013). Theoretical convergence in assessment of cognition. Journal of Psychoeducational Assessment, 31, 148-156.

Brody, N. (1999). What is intelligence? International Review of Psychiatry, 11, 19-25.

Beck, A. T., Steer, R. A., \& Brown, G. K. (1996). Manual for the Beck Depression Inventory-II. San Antonio, TX: Psychological Corporation.

Burdenski, T. (2000). Evaluating univariate, bivariate, and multivariate normality using graphical and statistical procedures. Multiple Linear Regression Viewpoints, $26,15-28$.

Brophy, C. J., Norvell, N. K., \& Kiluk, D. J. (1988). An examination of the factor structure and convergent and discriminant validity of the SCL-90R in an outpatient clinic population. Journal of Personality Assessment, 52, 334-340. Buffum, A., Mattos, M., \& Weber, C. (2010). The why try behind RTI: Response to Intervention flourishes when educators implement the right practices for the right reasons. Educational Leadership, Oct. 2010, 10-16.

Canivez, G. L. (2011). Hierarchical factor structure of the Cognitive Assessment System: Variance partitions from the Schmid-Leiman (1957) procedure. School Psychology Quarterly, 26, 305-317.

Canivez, G. L. (2013). Psychometric versus actuarial interpretation of intelligence and related aptitude batteries. In D. H. Saklofske, C. Reynolds, \& V. L. Schwean (Eds.), Oxford handbook of psychological assessment of children and adolescents (pp. 84-112). New York: Oxford University Press. 
Canivez, G. L., \& Watkins, M. W. (2010). Investigation of the factor structure of the Wechsler Adult Intelligence Scale - Fourth Edition (WAIS-IV): Exploratory and higher order factor analyses. Psychological Assessment, 22, 827-836.

Caroll, J. (1990). Cognitive Abilities in Foreign Language Aptitude: Then and Now. In T. S. Parry \& C. W. Stansfield (Eds.), Language aptitude reconsidered (pp. 11-29). Englewood Cliffs. NJ: Prentice Hall.

Carroll, J. B. (1993). Human cognitive abilities. Cambridge, United Kingdom: Cambridge University Press.

Carroll, J. B. (2012). The three-stratum theory of cognitive abilities. In D. P. Flanagan \& P. L. Harrison (Eds.), Contemporary intellectual assessment: Theories, tests, and issues $\left(3^{\text {rd }}\right.$ ed., pp. 883-890). New York: Guilford Press.

Castaneda, A. E., Tuulio-Henriksson, A., Marttunen, M., Suvisaari, J., \& Lonnqvist, J. (2008). A review on cognitive impairments in depressive and anxiety disorders with a focus on young adults. Journal of Affective Disorders, 106, 1-27.

Cattell, J. M. (1890). Mental tests and measurements. Mind, 15, 373-381.

Cattell, R. B. (1943). The measurement of adult intelligence. Psychological Bulletin, 40, 153-193.

Cattell, R. B. (1963). Theory of fluid and crystallized intelligence: A critical experiment. Journal of Educational Psychology, 54, 1-22.

Cattell, R. B. (1966). The scree test for the number of factors. Multivariate Behavioral Research, 1, 629-637.

Cattell, R. B. (1987). Intelligence: Its structure, growth and action. Amsterdam: NorthHolland.

Cirino, P. T., Morris, M. K., \& Morris, R. D. (2002). Neuropsychological concomitants of calculation skills in college students referred for learning difficulties.

Developmental Neuropsychology, 21, 201-218.

Ciszek, G. J. (2003). Review of the Woodcock-Johnson III. In B. S. Plake \& J. C. Impara (Eds.), The fifteenth mental measurements yearbook (pp. 1020-1024). Lincoln, NE: Buros Institute of Mental Measurements.

Claeys, G. (2001). Introducing Francis Galton, 'Kantsaywhere' and 'The Donoghues of Dunno Weir.' Utopian Studies, 12, 188-190. 
Compton, D. L., Fuchs, L. S., Fuchs, D., Lambert, W., \& Hamlett, C. (2012). The cognitive and academic profiles of reading and mathematics learning disabilities. Journal of Learning Disabilities, 45, 79-95.

Conners, C., Erhardt, D., \& Sparrow, E. (1998). The Conners Adult ADHD Rating Scale. Toronto, Canada: Multi-Health Systems, Inc.

Conners, C. K., Erhardt, D., \& Sparrow, E. (1999). Conners' Adult ADHD Rating Scales: Technical manual. New York: Multi-Health Systems.

Conners, C. K., Erhardt, D., Epstein, J. N., Parker, J. D. A., Sitarenios, G., \& Sparrow, E. (1999). Self-ratings of ADHD symptoms in adults I: Factor structure and normative data. Journal of Attention Disorders, 3, 141-151.

Cornish, J. A. E., Gorgens, K. A., Monson, S. P., Olkin, R., Palombi, B. J., \& Abels, A. V. (2008). Perspectives on ethical practice with people who have disabilities. Professional Psychology: Research and Practice, 39, 488-497.

Cory, R. C. (2011). Disability services offices for students with disabilities: A campus resource. New Directions for Higher Education, 154, 27-36.

Das, J. P., Naglieri, J. A., \& Kirby, J. R. (1994). Assessment of Cognitive Processes: The PASS Theory of Intelligence. Needham Heights, MA: Allyn \& Bacon.

DeFife, J.A., Peart, J., Bradley, B., Ressler, K., Drill, R., Beinashowitz, J., \& Westen, D. (2013). The validity of prototype diagnosis for mood and anxiety disorders. Journal of the American Medical Association - Psychiatry, 70, 140-148. doi: 10.1001/jamapsychiatry.2013.270

DeFries, J. C., Vandenberg, S. G., McClearn, G. E., Kuse, A. R., Wilson, J. R., Ashton, G. G., \& Johnson, R. C. (1974). Near identity of cognitive structure in two ethnic groups. Science, 183, 338-339.

Derogatis, L. R. (1994). Symptom Checklist-90-R (SCL-90-R): Administration, scoring and procedures manual - third edition. Minneapolis, MN: NCS Pearson, Inc.

Drozdick, L. W., Wahlstrom, D., Zhu, J., \& Weiss, L. G. (2012). The Wechsler Adult Intelligence Scale - Fourth Edition and the Wechsler Memory Scale - Fourth Edition. In D. Flanagan \& P. Harrison (Eds.), Contemporary Intellectual Assessment: Theories, Tests, and Issues ( $3^{\text {rd }}$ ed.). New York: Guilford. 
Duckworth, A. L., Quinn, P. D., \& Tsukayama, E. (2011). What no child left behind leaves behind: The roles of IQ and self-control in predicting standardized achievement test scores and report card grades. Journal of Educational Psychology, 104, 439-451.

DuPaul, G. J., Weyandt, L. L., O’Dell, S. M., \& Varejao, M. (2009). College students with ADHD: Current status and future directions. Journal of Attention Disorders, 13, 234-250.

Eisenberg, D., Golberstein, E., \& Gollust, S. E. (2007). Help-seeking and access to mental health care in a university student population. Medical Care, 45, 594-601.

Fabrigar, L. R., Wegener, D. T., MacCullum, R. C., \& Strahan, E. J. (1999). Evaluating the use of exploratory factor analysis in psychological research. Psychological Methods, 4, 272-299.

Ferrari, M., \& Palladino, P. (2007). Foreign language learning difficulties in Italian children: Are they associated with other learning difficulties? Journal of Learning Disabilities, 40, 256-269.

Ferrer, E., \& McArdle, J. J. (2004). An experimental analysis of dynamic hypotheses about cognitive abilities and achievement from childhood to early adulthood. Developmental Psychology, 40, 935-952.

Finke, K., Schwarzkopf, W., Muller, U., Frodl, T., Muller, H. J., Schneider, W. X., Engel, R. R., ...Hennig-Fast, K. (2011). Disentangling the adult attention-deficit hyperactivity disorder endophenotype: Parametric measurement of attention. Journal of Abnormal Psychology, 120, 890-901.

First, M. B., Spitzer, R. L., Gibbon M., and Williams, J. B. W. (2002). Structured Clinical Interview for DSM-IV-TR Axis I Disorders, Research Version, Patient Edition With Psychotic Screen (SCID-I/P W/ PSY SCREEN). New York: Biometrics Research, New York State Psychiatric Institute.

Fiorello, C. A., Hale, J. B., Holdnack, J. A., Kavanagh, J. A., Terrell, J., \& Long, L. (2007). Interpreting intelligence test results for children with disabilities: Is global intelligence relevant? Applied Neuropsychology, 14, 2-12.

Flanagan, D. P., Alfonso, V. C., \& Ortiz, S. O. (2012). The cross-battery assessment approach: An overview, historical perspective, and current directions. In D. P. Flanagan \& P. L. Harrison (Eds.), Contemporary intellectual assessment: Theories, tests, and issues ( $3^{\text {rd }}$ ed., pp. 459-483). New York: Guilford Press. 
Flanagan, D. P., Fiorello, C. A., \& Ortiz, S. O. (2010). Enhancing practice through application of Cattell-Horn-Carroll theory and research: A "third method" approach to specific learning disability identification. Psychology in the Schools, 47, 739-760.

Flanagan, D. P., \& Mascolo, J. T. (2005). Psychoeducational assessment and learning disability diagnosis. In D. P. Flanagan \& P. L. Harrison (Eds.), Contemporary intellectual assessment: Theories, tests, and issues ( $2^{\text {nd }}$ ed., pp. 371-401). New York: Guilford Press.

Flanagan, D. P., Ortiz, S. O., \& Alfonso, V. C. (2007). Essentials of cross-battery assessment, second edition. Hoboken, NJ: John Wiley and Sons.

Floyd, R. G., McGrew, K. S., Barry, A., Rafael, F., \& Rogers, J. (2009). General and specific effects on Cattell-Horn-Carroll broad ability composites: Analysis of the Woodcock-Johnson III Normative Update Cattell-Horn-Carroll factor clusters across development. School Psychology Review, 38, 249-265.

Floyd, R. G., McGrew, K. S., \& Evans, J. J. (2008). The relative contributions of the Cattell-Horn-Carroll cognitive abilities in explaining writing achievement during childhood and adolescence. Psychology in the Schools, 45, 132-144.

Floyd, R. G., Shands, E. I., Fawziya, A. R., Bergeron, R., McGrew, K. S. (2009). The dependability of general-factor loadings: The effects of factor-extraction methods, test battery composition, test battery size, and their interactions. Intelligence, 37, 453-465.

Flynn J. R. (1987). "Massive IQ gains in 14 nations: What IQ tests really measure". Psychological Bulletin, 101, 171-191.

Francomano, A., Bonanno, B., Fuca, L., La Placa, M., \& La Barbera, D. (2011). The role of antidepressant treatments on cognitive deficits: A review of recent literature. Clinical Neuropsychiatry, 8, 354-366.

Frazier, T. W., Youngstrom, E. A., Glutting, J. J., \& Watkins, M. W. (2007). ADHD and achievement: Meta-analysis of the child, adolescent, and adult literatures and a concomitant study with college students. Journal of Learning Disabilities, 40, 4965 .

Freberg, M. E., Vandiver, B. J., Watkins, M. W., \& Canivez, G. L. (2008). Significant factor score variability and the validity of the WISC-III Full scale IQ in predicting later academic achievement. Applied Neuropsychology, 15, 131-139. 
Fuchs, D., \& Fuchs, L. S. (2006). Introduction to response to intervention: What, why, and how valid is it? Reading Research Quarterly, 41, 93-99.

Gaudino, E. A., Geisler, M. W., \& Squires, N. K. (1995). Construct validity in the Trail Making Test: What makes Part B harder? Journal of Clinical and Experimental Neuropsychology, 17, 529-535.

Geary, D. C. (1993). Mathematical disabilities: Cognitive, neuropsychological, and genetic components. Psychological Bulletin, 114, 345-362.

Gilabert, R., \& Munoz, C. (2010). Differences in attainment and performance in a foreign language: The role of working memory capacity. International Journal of English Studies, 10, 19-42.

Glutting, J., Adams, W., \& Sheslow, D. (2000). Wide Range Intelligence Test. Wilmington, DE: Wide Range, Inc.

Glutting, J. J., \& McDermott, P. A. (1994). Core profile types for the WISC-III and WIAT: Their development and application in identifying multivariate IQachievement discrepancies. School Psychology Review, 23, 619-639.

Glutting, J. J., McDermott, P. A., \& Stanley, J. C. (1987). Resolving differences among methods of establishing confidence limits for test scores. Educational and Psychological Measurement, 47, 607-614.

Glutting, J. J., McGrath, E. A., Kamphaus, R. W., \& McDermott, P. A. (1992). Taxonomy and validity of the subtest profiles on the Kaufman Assessment Battery for Children. The Journal of Special Education, 26, 85-115.

Glutting, J. J., \& Watkins, M. W. (1997). The base rate problem and its consequences for interpreting children's ability profiles. School Psychology Review, 26, 176-189.

Glutting, J. J., Watkins, M. W., Konold, T. R., \& McDermott, P. A. (2006). Distinctions without a difference: The utility of observed versus latent factors from the WISCIV in estimating reading and math achievement on the WIAT-II. The Journal of Special Education, 40, 103-114.

Glutting, J. J., Youngstrom, E. A., Ward, T., Ward, S., \& Hale, R. L. (1997). Incremental efficacy of WISC-III factor scores in predicting achievement: What do they tell us? Psychological Assessment, 9, 295-301.

Glutting, J. J., Youngstrom, E. A., \& Watkins, M. W. (2005). ADHD and college students: Exploratory and confirmatory factor structures with student and parent data. Psychological Assessment, 17, 44-55. 
Golay, P., \& Lecerf, T. (2011). Orthogonal higher order structure and confirmatory factor analysis of the French Wechsler Adult Intelligence Scale (WAIS-III).

Psychological Assessment, 23, 143-152.

Golay, P., Reverte, I., Rossier, J., Favez, N., \& Lecerf, T. (2012, November 12). Further insights on the French WISC-IV factor structure through Bayesian structural equation modeling. Psychological Assessment. Advance online publication. doi: $10.1037 / \mathrm{a} 0030676$

Goldman, H.H., Rye, P., \& Sirovatka, P. (Eds.) (1999). Mental health: A report of the surgeon general. Retrieved September 28, 2012 from http://www. surgeongeneral.gov/library/mentalhealth/home.html.

Goldstein, S., \& Naglieri, J. A. (2008). The school neuropsychology of ADHD: Theory, assessment, and intervention. Psychology in the Schools, 45, 859-874.

Gregg, N., Coleman, C., Davis, M., Lindstrom, W., \& Hartwig, J. (2006). Critical issues for the diagnosis of learning disabilities in the adult population. Psychology in the Schools, 43, 889-899.

Gropper, R. J., \& Tannock, R. (2009). A pilot study of working memory and academic achievement in college students with ADHD. Journal of Attention Disorders, 12, 574-581.

Hadley, W. M. (2011). College students with disabilities: A student development perspective. New Directions for Higher Education, 154, 77-81.

Hakstian, A. R., \& Cattell, R. B. (1975). The Comprehensive Ability Battery. Champaign: Institute for Personality and Ability Testing.

Hale, J. B., Fiorello, C. A., Dumont, R., Willis, J. O., Rackley, C., \& Elliott, C. (2008). Differential Ability Scales-Second Edition (neuro)psychological predictors of math performance for typical children and children with math disabilities. Psychology in the Schools, 45, 838-858.

Hartley, M. T. (2010). Increasing resilience: Strategies for reducing dropout rates for college students with psychiatric disabilities. American Journal of Psychiatric Rehabilitation, 13, 295-315.

Hayton, J. C., Allen, D. G., \& Scarpello, V. (2004). Factor retention decisions in exploratory factor analysis: A tutorial on parallel analysis. Organizational Research Methods, 7, 191-205. 
Henson, R. K., \& Roberts, J. K. (2006). Use of exploratory factor analysis in published research: Common errors and some comment on improved practice. Educational and Psychological Measurement, 66, 393-416.

Hoover, H. D., Dunbar, S. B., Frisbie, D. A., Oberley, K. R., Bray, G. B., Naylor, R. J., et al. (2003). Iowa Tests of Basic Skills Complete/Core Battery: Norms and Score Conversions: Student Norms and School Average Norms. Itasca, IL: Riverside Publishing.

Horn, J. L., \& Blankson, A. N. (2012). Foundations for better understanding of cognitive abilities. In D. P. Flanagan and P. L. Harrison (Eds.), Contemporary intellectual assessment: Theories, tests, and issues ( $3^{\text {rd }}$ ed., pp. 73-98). New York: Guilford Press.

Horn, J. L., \& Cattell, R. B. (1966). Refinement and test of the theory of fluid and crystallized intelligence. Journal of Educational Psychology, 57, 253-270.

Horn, J. L., \& Cattell, R. B. (1982). Whimsy and misunderstandings of Gf-Gc theory: A comment on Guilford. Psychological Bulletin, 91, 623-633.

Hu, L., \& Bentler, P. M. (1999). Cutoff criteria for fit indexes in covariance structure analysis: Conventional criteria versus new alternatives. Structural Equation Modeling, 6, 1-55.

Individuals with Disabilities Education Improvement Act of 2004 (IDEA 2004), Pub. L. No., 108-446.

Jensen, A. R. (1998). The g factor. Westport, CT: Praeger.

Johnson, W., Bouchard, T. J., Jr., Krueger, R. F., McGue, M., \& Gottesman, I. I. (2004). Just one g: Consistent results from three test batteries. Intelligence, 32, 95-107.

Kahana, S. Y., Youngstrom, E. A., \& Glutting, J. J.(2002). Factor and subtest discrepancies on the Differential Ability Scales: Examining prevalence and validity in predicting academic achievement. Assessment, 9, 82-93.

Kaiser, H. F. (1960). The application of electronic computers to factor analysis. Educational and Psychological Measurement, 20, 141-151.

Kamphaus, R. W., Winsor, A. P., Rowe, W. W., \& Kim, S. (2005). A history of intelligence test interpretation. In D. P. Flanagan \& P. L. Harrison (Eds.), Contemporary intellectual assessment: Theories, tests, and issues $\left(2^{\text {nd }}\right.$ ed., pp. 2338). New York: Guilford Press. 
Kan, K. J., Kievit, R. A., Dolan, C., \& van der Maas, H. (2011). On the interpretation of the CHC factor Gc. Intelligence, 39, 292-302.

Keith, T. Z., \& Reynolds, M. R. (2010). Cattell-Horn-Carroll abilities and cognitive tests: What we've learned from 20 years of research. Psychology in the Schools, 47, $635-650$.

Kaufman, A. S. (1994). Intelligent testing with the WISC-III. New York: Wiley.

Kaufman, A. S., \& Kaufman, N. L. (2004a). Kaufman Assessment Battery for ChildrenSecond Edition (K-ABC-II). Circle Pines, MN: American Guidance Service.

Kaufman, A. S., \& Kaufman, N. L. (2004b). Kaufman Test of Educational AchievementSecond Edition (KTEA-II). Comprehensive form. Circle Pines, MN: American Guidance Service.

Kaufman, S. B., Reynolds, M. R., Liu, X., Kaufman, A. S., \& McGrew, K. S. (2012). Are cognitive $g$ and academic achievement $g$ one and the same $g$ ? An exploration on the Woodcock-Johnson and Kaufman tests. Intelligence, 40, 123-138.

Keith, T. Z., Kranzler, J. H., \& Flanagan, D. P. (2001). What does the Cognitive Assessment System measure? A joint confirmatory factor analysis of the CAS and Woodcock-Johnson Tests of Cognitive Ability (3rd Edition). School Psychology Review, 30, 89-119.

Konold, T. R., \& Canivez, G. L. (2010). Differential relationships between WISC-IV and WIAT-II scales: An evaluation of potentially moderating child demographics. Educational and Psychological Measurement, 70, 613-627.

Konold, T. R., Glutting, J. J., \& McDermott, P. A. (1997). The development and applied utility of a normative aptitude-achievement taxonomy for the Woodcock-Johnson Psycho-Educational Battery-Revised. The Journal of Special Education, 31, 212232.

Kotz, K. M., Watkins, M. W., \& McDermott, P. A. (2008). Validity of the general conceptual ability score from the Differential Ability Scales as a function of significant and rare interfactor variability. School Psychology Review, 37, 261278.

Kraft, D. P. (2010). Book review: Nonmedication treatments for adult ADHD evaluating impact on daily functioning and well-being, by J. Russell Ramsay, PhD. Journal of American College Health, 59, 57-59. 
Kranzler, J. H. (1997). Education and policy issues related to the use and interpretation of intelligence tests in the schools. School Psychology Review, 26, 150-162.

Lichtenberger, E., \& Kaufman, A. (2009). Essentials of WAIS-IV assessment. Hoboken, NJ: Wiley.

MacCallum, R. C., Widaman, K. F., Zhang, S., \& Hong S. (1999). Sample size in factor analysis. Psychological Methods, 4, 84-99.

Maller, S. J., \& McDermott, P. A. (1997). The WAIS-R profile analysis for college students with learning disabilities. School Psychology Review, 26, 575-585.

Manalo, E., Ede, J., \& Wong-Toi, G. (2010). Provision of learning support for university students with learning, mental health, and other forms of hidden disabilities. The Open Rehabilitation Journal, 23-33.

Mather, N., \& Woodcock, R. W. (2001a). Examiner's Manual. Woodcock-Johnson III Tests of Achievement. Itasca, IL: Riverside Publishing.

Mather, N., \& Woodcock, R. W. (2001b). Examiner's Manual. Woodcock-Johnson III Tests of Cognitive Abilities. Itasca, IL: Riverside Publishing.

Mather, N., \& Woodcock, R. W. (2001c). Technical Manual. Woodcock-Johnson III Tests of Cognitive Abilities. Itasca, IL: Riverside Publishing.

McCloskey, G., Whitaker, J., Murphy, R., \& Rogers, J. (2012). Intellectual, cognitive, and neuropsychological assessment in three-tier service delivery systems in schools. In D. P. Flanagan \& P. L. Harrison (Eds.), Contemporary intellectual assessment: Theories, tests, and issues ( $3^{\text {rd }}$ ed., pp. 852-881). New York: Guilford Press.

McDermott, P. A., Fantuzzo, J. W., Glutting J. J., Watkins, M. W., \& Baggaley, A. R. (1992). Illusions of meaning in the ipsative assessment of children's ability. The Journal of Special Education, 25, 504-526.

McGrew, K. S., Schrank, F. A., \& Woodcock, R. W. (2007). Technical Manual. Woodcock-Johnson III Normative Update. Rolling Meadows, IL: Riverside Publishing.

McGrew, K. S. (1997). Analysis of the major intelligence batteries according to a proposed comprehensive $G f-G c$ framework. In D. P. Flanagan, J. L. Genshaft, \& P. L. Harrison (Eds.), Contemporary intellectual assessment: Theories, tests, and issues (pp. 151-179). New York: Guilford. 
McGrew, K. S. (2005). The Cattell-Horn-Carroll theory of cognitive abilities: Past, present, and future. In D. P. Flanagan \& P. L. Harrison (Eds.), Contemporary intellectual assessment: Theories, tests, and issues ( $2^{\text {nd }}$ ed., pp. 136-181) New York: Guilford Press.

McGrew, K. S. (2009). CHC theory and the human cognitive abilities project: Standing on the shoulders of the giants of psychometric intelligence research. Intelligence, $37,1-10$.

McGrew, K. S. (2011). CHC narrow ability assessment with the WJ III battery. Retrieved from http://www.iapsych.com/iapap101/iapap10112.pdf.

McGrew, K. S., \& Flanagan, D. P. (1998). The intelligent test desk reference (ITDR): GfGc cross-battery assessment. Boston: Allyn \& Bacon.

McGrew, K. S., Flanagan, D. P., Keith, T. Z., \& Vanderwood, M. (1997). Beyond g: The impact of $G f-G c$ specific cognitive abilities research on the future use and interpretation of intelligence tests in the schools. School Psychology Review, 26, 189-210.

McGrew, K. S., \& Wendling, B. J. (2010). Cattell-Horn-Carroll cognitive-achievement relations: What we have learned from the past 20 years of research. Psychology in the Schools, 47, 651-675.

McGrew, K. S., \& Woodcock, R. W. (2001). Woodcock-Johnson III technical manual. Itasca, IL: Riverside Publishing.

McLean, A., Dowson, J., Toone, B., Young, S., Bazanis, E. Robbins, T. W., \& Sahakian, B. J. (2004). Characteristic neurocognitive profile associated with adult attentiondeficit/hyperactivity disorder. Psychological Medicine, 34, 681-692.

Mrazik, M., Bender, S., \& Makovichuk, C. (2010). Memory functioning in postsecondary students with learning disabilities. Research in Higher Education Journal, 1-9.

Murray, C., \& Wren, C. T., (2003). Cognitive, academic, and attitudinal predictors of the grade point averages of college students with learning disabilities. Journal of Learning Disabilities, 36, 407-415.

Murray, C., Lombardi, A., \& Wren, C. T. (2011). The effects of disability-focused training on the attitudes and perceptions of university staff. Remedial and Special Education, 32, 290-300. 
Naglieri, J. A., \& Bornstein, B. T. (2003). Intelligence and achievement: Just how correlated are they? Journal of Psychoeducational Assessment, 21, 244-260.

Naglieri, J. A., \& Das, J. P. (1997a). Cognitive Assessment System. Itasca, IL: Riverside.

Naglieri, J. A., \& Das, J. P. (1997b). Interpretive handbook for the Cognitive Assessment System. Itasca, IL: Riverside.

National Joint Committee on Learning Disabilities (2007). Comprehensive assessment and evaluation of students with learning disabilities. Retrieved October 25, 2012 from http://www.ldonline.org/about/partners/njcld/.

National Joint Committee on Learning Disabilities (2007). The documentation disconnect for students with learning disabilities: Improving access to postsecondary disability services. Retrieved October 25, 2012 from http://www.ldonline.org/about/ partners/njcld/.

Nelson, J. M., \& Canivez, G. L. (2012). Examination of the structural, convergent, and incremental validity of the Reynolds Intellectual Assessment Scales (RIAS) with a clinical sample. Psychological Assessment, 24, 129-140.

Nigg, J. T., Stavro, G., Ettenhofer, M., Hambrick, D. Z., Miller, T., \& Henderson, J. M. (2005). Executive functions and ADHD in adults: Evidence for selective effects on ADHD symptom domains. Journal of Abnormal Psychology, 114, 706-717.

Norvilitis, J. M., Sun, L., \& Zhang, J. (2009). AHDH symptomatology and adjustment to college in China and the United States. Journal of Learning Disabilities, 43, 8694.

Office of Special Education Programs (OSEP), U.S. Department of Education. Building the legacy: IDEA 2004. Retrieved September 26, 2012 from http://idea.ed. gov/explore/home.

Ofiesh, N. S. (2000). Using processing speed tests to predict the benefit of extended test time for university students with learning disabilities. Journal of Postsecondary Education and Disability, 14, 39-56.

Oh, H. J., Glutting, J. J., Watkins, M. W., Youngstrom, E. A., \& McDermott, P. A. (2004). Correct interpretation of latent versus observed abilities: Implications from structural equation modeling applied to the WISC-III and WIAT linking sample. The Journal of Special Education, 38, 159-173. 
Orr, A. C., \& Hammig, S. B. (2009). Inclusive postsecondary strategies for teaching students with learning disabilities: A review of the literature. Learning Disability Quarterly, 32, 181-196.

Osmon, D. C., Braun, M. M., \& Plambeck, E. A. (2005). Processing abilities associated with phonologic and orthographic skills in adult learning disability. Journal of Clinical and Experimental Neuropsychology, 27, 544-554.

Osmon, D. C., Smerz, J. M., Braun, M. M., \& Plambeck, E. (2006). Processing abilities associated with math skills in adult learning disability. Journal of Clinical and Experimental Neuropsychology, 28, 84-95.

Parker, D. R., \& Boutelle, K. (2009). Executive function coaching for college students with learning disabilities and ADHD: A new approach for fostering selfdetermination. Learning Disabilities Research \& Practice, 24, 204-215.

Parkin, J. R., \& Beaujean, A. A. (2012). The effects of Wechsler intelligence scale for children - fourth edition cognitive abilities on math achievement. Journal of School Psychology, 50, 113-128.

Phelps, L., McGrew, K. S., Knopik, S. N., \& Ford, L. (2005). The general (g), broad, and narrow CHC stratum characteristics of the WJ III and WISC-IV tests: A confirmatory cross-battery investigation. School Psychology Quarterly, 20, 66-88.

Plucker, J. A. (Ed.). (2003). Human intelligence: Historical influences, current controversies, teaching resources. Retrieved September 5, 2012, from http://www.indiana.edu/ intell.

Prevatt, F., Welles, T. L., Huijun, L., \& Proctor, B. (2010). The contribution of memory and anxiety to the math performance of college students with learning disabilities. Learning Disabilities Research \& Practice, 25, 39-47.

Proctor, B. (2012). Relationships between Cattell-Horn-Carroll (CHC) cognitive abilities and math achievement with a sample of college students with learning disabilities. Journal of Learning Disabilities, 45, 278-287.

Proctor, B. E., Floyd, R. G., \& Shaver, R. B. (2005). Cattell-Horn-Carroll broad cognitive ability profiles of low math achievers. Psychology in the Schools, 42, 1-12.

Psychological Corporation. (1999). Wechsler Abbreviated Scale of Intelligence manual. San Antonio, TX: Author.

R Development Core Team (2008). R: A language and environment for statistical computing. R Foundation for Statistical Computing, Vienna, Austria. ISBN 3900051-07-0, URL http://www.R-project.org. 
Raue, K., and Lewis, L. (2011). Students with Disabilities at Degree-Granting Postsecondary Institutions (NCES 2011-018). U.S. Department of Education, National Center for Education Statistics. Washington, DC: U.S. Government Printing Office.

Raven, J. C. (1941). Standardization of progressive matrices, 1938. British Journal of Medical Psychology, 19, 137-150.

Raven, J., Raven, J. C., \& Court, J. H. (1998). Manual for Raven's Progressive Matrices and Vocabulary Scales: Section 5 Mill Hill vocabulary scale, 1998 ed. Oxford, UK: Oxford Psychologists Press Ltd.

Reaser, A., Prevatt, F., Petscher, Y., \& Proctor, B. (2007). The learning and study strategies of college students with ADHD. Psychology in the Schools, 44, 627638.

Reise, S. P., Moore, T. M., \& Haviland, M. G. (2010). Bifactor models and rotations: Exploring multidimensional data yield univocal scale scores. Journal of Personality Assessment, 92, 544-559. doi:10.1080/00223891.2010.49647

Reitan, R. M. (1986). Trail Making Test: Manual for administration and scoring. Tucson, AZ: Reitan Neuropsychology Laboratory.

Response to intervention: Remediation, perhaps, diagnosis, no. Child Development Perspectives, 3, 44-47.

Reynolds, C. R., \& Kamphaus, R. W. (2003). Reynolds Intellectual Assessment Scales. Lutz, FL: Psychological Assessment Resources Inc. Reynolds, C. R., \& Shaywitz, S. E. (2009).

Reynolds, C. R., \& Shaywitz, S. E. (2009). Response to intervention: Ready or not? Or, from wait-to-fail to watch-them-fail. School Psychology Quarterly, 24, 130-145.

Reynolds, M. R., Hajovsky, D. B., Niileksela, C. R., \& Keith, T. Z., (2011). Spearman's law of diminishing returns and the DAS-II: Do $g$ effects on subtest scores depend on the level of $g$ ? School Psychology Quarterly, 26, 275-289.

Reynolds, M. R., Keith, T. Z., Flanagan, D. P., \& Alfonso, V. C. (2013). A cross-battery, reference variable, confirmatory factor analytic investigation of the $\mathrm{CHC}$ taxonomy. Journal of School Psychology, 2013), http://dx.doi.org/ 10.1016/j.jsp.2013.02.003. 
Revelle, W. (2013) psych: Procedures for personality and psychological research, Northwestern University, Evanston, Illinois, USA, http://CRAN.Rproject.org/package $=$ psych Version $=1.3 .2$.

Robinson, P. P. (2001). Individual differences, cognitive abilities, aptitude complexes and learning conditions in second language acquisition. Second Language Research, 17(4), 368-392.

Rohde, T. E., \& Thompson, L. A. (2006). Predicting academic achievement with cognitive ability. Intelligence, 35, 83-92.

Roid, G. H. (2003). Stanford-Binet Intelligence Scales, Fifth Edition. Itasca, IL: Riverside.

Rosseel, Y. (2012). lavaan: An R package for structural equation modeling. Journal of Statistical Software, 48, 1-36. URL http://www.jstatsoft.org/v48/i02/

Rysiewicz, J. (2008). Cognitive Profiles of (Un)successful FL Learners: A Cluster Analytical Study. The Modern Language Journal, 92(1), 87-99.

Sanders, S., McIntosh, D. E., Dunham, M., Rothlisberg, B. A., \& Finch, H. (2007). Joint confirmatory factor analysis of the Differential Ability Scales and the WoodcockJohnson Tests of Cognitive Abilities-Third Edition. Psychology in the Schools, 44, 119-138.

Sandoval, J. (2003). Review of the Woodcock-Johnson III. In B. S. Plake \& J. C. Impara (Eds.), The fifteenth mental measurements yearbook (pp 1024-1028). Lincoln, NE: Buros Institute of Mental Measurements.

Sasaki, M. (2012). The Modern Language Aptitude Test (Paper-and-Pencil Version). Language Testing, 29(2), 315-321.

Schmid, J., \& Leiman, J. M. (1957). The development of hierarchical factor solutions. Psychometrika, 22, 53-61.

Schmitz, N., Hartkamp, N., Kruse, J., Franke, G. H., Reister, G., \& Tress, W. (2000). The Symptom Checklist-90-R (SCL-90-R): a German validation study. Quality of Life Research, 9, 185-193.

Schmitz, N., Kruse, J., Heckrath, C., Alberti, L., \& Tress, W. (1999). Diagnosing mental disorders in primary care: The General Health Questionnaire (GHQ) and the Symptom Checklist (SCL-90-R) as screening instruments. Social Psychiatry and Psychiatric Epidemiology, 34, 360-366. 
Schneider, W. J. (2008). Playing statistical ouija board with commonality analysis: Good questions, wrong assumptions. Applied Neuropsychology, 15, 44-53.

Schneider, W. J. (2011, July 7). Within-composite differences: Why measures of the same ability differ. [Web log post] Retrieved from http://assessingpsyche. wordpress.com/2011/07/07/within-composite-differences-why-measures-of-thesame-ability-differ/.

Schneider, W. J. (2011, July 15). Do large subtest score differences invalidate composite scores? [Web log post] Retrieved from http://assessingpsyche. wordpress.com/2011/07/15/do-large-subtest-score-differences-invalidatecomposite-scores/.

Schneider, W. J. (2013). Principles of assessment of aptitude and achievement. In D. Saklofske, C. Reynolds, \& V. Schwean (Eds.), Oxford handbook of psychological assessment of children and adolescents (pp.286-330). New York: Oxford University Press.

Schneider, W. J., and McGrew, K. S. (2012). The Cattell-Horn-Cattell model of intelligence. In D. P. Flanagan and P. L. Harrison (Eds.) Contemporary intellectual assessment: Theories, tests, and issues ( ${ }^{\text {rd }}$ ed., pp. 99-144). New York: Guilford Press.

Schrank, F. A. (2005). Woodcock-Johnson III tests of cognitive abilities. In D. P. Flanagan \& P. L. Harrison (Eds.), Contemporary intellectual assessment: Theories, tests, and issues ( $2^{\text {nd }}$ ed., pp. 371-401) New York: Guilford Press.

Schrank, F. A., \& Wendling, B. J. (2009). Educational interventions and accommodations related to the Woodcock-Johnson III Tests of cognitive Abilities and the Woodcock-Johnson III Diagnostic Supplement to the Tests of Cognitive Abilities Woodcock-Johnson III Assessment Service Bulletin No. 10. Rolling Meadows, IL: Riverside Publishing.

Schrank, F. A., \& Wendling, B. J. (2012). The Woodcock-Johnson III normative update: Tests of cognitive abilities and tests of achievement. In D. P. Flanagan and P. L. Harrison (Eds.) Contemporary intellectual assessment: Theories, tests, and issues ( $3^{\text {rd }}$ ed., pp. 297-335). New York: Guilford Press.

Scott, K., Bell, S., \& McCallum, R. (2009). Relation of native-language reading and spelling abilities to attitudes toward learning a second language. Preventing School Failure, 54, 30-40. 
Shang, C. Y., \& Gau, S. S. (2011). Visual memory as a potential cognitive endophenotype of attention deficit hyperactivity disorder. Psychological Medicine, 41, 2603-2614.

Silva, J. M., \& White, L. A. (1993). Relation of cognitive aptitudes to success in foreign language training. Military Psychology, 5, 79-93.

Sparks, R., Patton, J., Ganschow, L., Humbach, N., \& Javorsky, J. (2006). Native language predictors of foreign language proficiency and foreign language aptitude. Annals of Dyslexia, 56, 129-160.

Spearman, C. (1904). "General intelligence": Objectively determined and measured. American Journal of Psychology, 15, 201-293.

Spencer, H. (1864). The principles of biology (Vol. 1). London: Williams \& Norgate.

Spinks, R., Arndt, S., Caspers, K., Yucuis, R., McKirgan, L. W., Pfalzgraf, C., et al. (2007). School achievement strongly predicts midlife IQ. Intelligence, 35, 563567.

Stodden, R. A., Brown, S. E., \& Roberts, K. (2011). Disability-friendly university environments: conducting a climate assessment. New Directions for Higher Education, 154, 83-92.

Stodden, R. A., Whelley, T., Chang, C., \& Harding, T. (2001). Current status of educational support provision to students with disabilities in postsecondary education. Journal of Vocational Rehabilitation, 16, 189-198.

Stuebing, K. K., Fletcher, J. M., Branum-Martin, L., \& Francis D. J. (2012). Evaluation of the technical adequacy of the three methods for identifying specific learning disabilities based on cognitive discrepancies. School Psychology Review, 41, 322 .

Taub, G. E., Floyd, R. G., Keith, T. Z., \& McGrew, K. S. (2008). Effects of general and broad cognitive abilities on mathematics achievement. School Psychology Quarterly, 25, 187-198.

Taub, G. E., \& McGrew, K. S. (2004). A confirmatory factor analysis of Cattell-HornCarroll theory and cross-age invariance of the Woodcock-Johnson tests of cognitive abilities III. School Psychology Quarterly, 19, 72-87.

Thoma, C. A., \& Getzel, E. E. (2005). "Self-determination is what it's all about": What post-secondary students with disabilities tell us are important considerations for success. Education and Training in Developmental Disabilities, 40, 234-242. 
Thurstone, L. L. (1938). Primary mental abilities (Psychometric Monographs, No. 1). Chicago: University of Chicago Press.

Trainin, G., \& Swanson, H. L. (2005). Cognition, metacognition, and achievement of college students with learning disabilities. Learning Disability Quarterly, 28, 261-272.

U. S. Department of Labor's Office of Disability Employment Policy Job Accommodation Network (2010). Accommodation and compliance series higher education accommodations: Students with mental health impairments. Accommodation and Compliance Series. Retrieved September 17, 2012 from http://askjan.org.

U.S. Government Accountability Office (2009). Report to the chairman, Committee on Education and Labor, House of Representatives: Higher education and disability education needs a coordinated approach to improve its assistance to schools in supporting students. (GAO Publication No. GAO-10-33). Retrieved September 10, 2012 from http://www. gao.gov/products/GAO-10-33.

Vallejo, M. A., Jordán, C. M., Diaz, M. I., Comeche, M. I., \& Ortega, J. (2007). Psychological assessment via the internet: A reliability and validity study of online (vs. paper-and-pencil) versions of the General Health Questionnaire-28 (GHQ-28) and the Symptom Checklist-90-Revised (SCL-90-R). Journal of Internet Medical Research, 9(1):e2.doi:10.2196/jmir.9.1.e2.

Vock, M., Preckel, F., \& Holling, H. (2011). Mental abilities and school achievement: A test of a mediation hypothesis. Intelligence, 39, 357-369.

Wasserman, J. D. (2012). A history of intelligence assessment: The unfinished tapestry. In D. P. Flanagan and P. L. Harrison (Eds.) Contemporary intellectual assessment: Theories, tests, and issues ( $3^{\text {rd }}$ ed., pp. 3-55). New York: Guilford Press.

Wasserman, J. D. \& Tulsky, D. S. (2005). A history of intelligence assessment. In D. P. Flanagan and P. L. Harrison (Eds.) Contemporary intellectual assessment: theories, tests, and issues ( $3^{\text {rd }}$ ed.) (pp. 3-22). New York: Guilford Press.

Watkins, M. W., \& Glutting, J. J. (2000). Incremental validity of WISC-III profile elevation, scatter, and shape information for predicting reading and math achievement. Psychological Assessment, 12, 402-408.

Watkins, M. W., Glutting, J. J., \& Lei, P. W. (2007). Validity of the full-scale IQ when there is significant variability among WISC-III and WISC-IV factor scores. Applied Neuropsychology, 14, 13-20. 
Watkins, M. W., Lei, P. W., \& Canivez, G. L. (2007). Psychometric intelligence and achievement: A cross-lagged panel analysis. Intelligence, 35, 59-68.

Webberman, A. L. (2011). Academic coaching to promote student success: An interview with Carol Carter. Journal of Developmental Education, 35(2), 18-20.

Wechsler, D. (1955). Manual for the Wechsler Adult Intelligence Scale. New York: The Psychology Corporation.

Wechsler, D. (1974). Wechsler Intelligence Scale for Children-Revised. New York: The Psychological Corporation.

Wechsler, D. (1981). Wechsler Adult Intelligence Scale-Revised. New York: The Psychological Corporation.

Wechsler, D. (1991). Wechsler Intelligence Scale for Children-Third Edition. San Antonio, TX: The Psychological Corporation.

Wechsler, D. (1997a). WAIS-III-WMS-III technical manual. San Antonio, TX: Psychological Corporation.

Wechsler, D. (1997b). Wechsler Adult Intelligence Scale-Third Edition San Antonio, TX: The Psychological Corporation.

Wechsler, D. (2003). Wechsler Intelligence Scale for Children-Fourth Edition. San Antonio, TX: Psychological Corporation.

Wechsler, D. (2008). Wechsler Adult Intelligence Scale_Fourth Edition. San Antonio, TX: Pearson.

Westen, D., Shedler, J., \& Bradley, R. (2006). A prototype approach to personality disorder diagnosis. American Journal of Psychiatry, 163, 846-856.

White, S. W., Ollendick, T. H., \& Gray, B. C. (2011). College students on the autism spectrum: Prevalence and associated problems. Autism, 15, 683-701.

Widiger, T. A., Mangine, S., Corbitt, E. M., Ellis, C. G., \& Thomas, G. V. (1995). Personality Disorder Interview-IV: A semistructured interview for the assessment of personality disorders. Odessa, FL: Psychological Assessment Resources.

Wilson, K. Getzel, E., \& Brown, T. (2000). Enhancing the post-secondary campus climate for students with disabilities. Journal of Vocational Rehabilitation, 14, 37-50. 
Wissler, C. (1901). The correlation of mental and physical tests. New York: [Press of the new era printing company, Lancaster, Pa.]

Woodcock, R.W., McGrew, K., \& Mather, N. (2001a). Woodcock-Johnson III Tests of Achievement. Itasca, IL: Riverside.

Woodcock, R.W., McGrew, K., \& Mather, N. (2001b). Woodcock-Johnson III Tests of Cognitive Ability. Itasca, IL: Riverside.

Woodcock, R. W., McGrew, K. S., \& Mather, N. (2007). Woodcock-Johnson III. Rolling Meadows, IL: Riverside.

Youngstrom, E. A., Kogos, J. L., \& Glutting, J. J. (1999). Incremental efficacy of differential ability scales factor scores in predicting individual achievement criteria. School Psychology Quarterly, 14, 26-39.

Zylowska, L., \& Siegel, D. (2012). The mindfulness prescription for adult ADHD: An 8step program for strengthening attention, managing emotions, and achieving your goals. Boston: Trumpeter Books. 